\title{
Campos Hamiltonianos e Colchete de Poisson na Teoria Geométrica dos Campos
}

\author{
Mário Otávio Salles
}

TESE APRESENTADA

$\mathrm{AO}$

INSTITUTO DE MATEMÁTICA E ESTATÍSTICA

DA

UNIVERSIDADE DE SÃO PAULO

PARA

OBTENÇÃO DO GRAU DE DOUTOR

EM

MATEMÁTICA APLICADA

\author{
Área de Concentração: Matemática Aplicada \\ Orientador: Prof. Dr. Frank Michael Forger
}

Durante a elaboração deste trabalho o autor recebeu apoio financeiro do $C N P q$

- São Paulo, 2 de dezembro de 2004 - 


\title{
Campos Hamiltonianos e Colchete de Poisson na Teoria Geométrica dos Campos
}

\author{
Este exemplar corresponde à redação final \\ da tese devidamente corrigida e defendida \\ por Mário Otávio Salles e aprovada pela \\ comissão julgadora.
}

São Paulo, 1 de dezembro de 2004.

Banca examinadora:

- Prof. Dr. Frank Michael Forger (Orientador) - IME - USP

- Prof. Dr. Paolo Piccione - IME - USP

- Prof. Dr. Jorge Passamani Zubelli - IMPA

- Prof. Dr. Sandro Vieira Romero - UFV

- Prof. Dr. Luiz Antonio Barrera San Martin - IMECC - UNICAMP 
à Anne, por tudo que ela significou, uma mãe. 


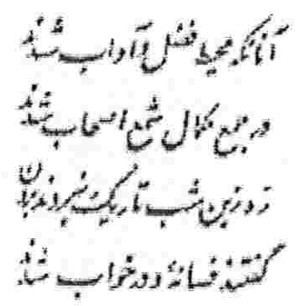

"Então do TI em MIM que busca atrás Do véu do Universo, chorei para encontrar Um Candeeiro que me guiasse na escuridão; $e$ Então alguma coisa disse - 'uma cega Inteligência'. "

Omar Khayyam - 1120 DC

Matemático persa.

Discípulo de Avicena. 


\section{Agradecimentos}

Gostaria de deixar aqui os nomes daquelas pessoas que estiveram presentes nestes anos na USP ou que então torceram pela realização deste trabalho.

À memória de meu pai Mário.

À minha mãe Anne, que buscou compreender minhas escolhas e sempre apoiou-me em minhas opções. A ela dedico este trabalho com amor.

À toda a minha família, que viu minha partida do Sul e acompanhou minha evolução que desdobra-se agora, em especial minha tia Altanisa, ao meu irmão Cristiano, ao meu irmão Diogo, à Valéria e claro, ao pequeno João Pedro, que acabou de chegar!

À Gisa, Marisa e Sabrina, pelo que representaram, consciente ou inconscientemente, e também pelos estímulos e entusiasmo, que ao seu tempo e à sua maneira marcaram meu caminho com suas presenças.

Aos Mestres Alcides de Lima e Dorival dos Santos e a todos meus irmãos de Capoeira por terem me acolhido na família CEACA (Centro de Estudos e Aplicação da Capoeira) e mostrado que "O segredo da Lua quem sabe é o clarão do Sol".

Aos amigos do CEOP - Centro de Educação e Organização Popular - e do Coletivo Griot, pela força e pela coragem de dizer não mesmo quando todos diziam sim.

Aos amigos e companheiros de "luta": Albetã, Alessandra, Cláudio, Daniel, Jutta e Beto, Max e Rogério.

Aos colegas de "trabalho": Bruno, Fernando, Leandro, Paola e Sandro, pelas discussões sempre agradáveis.

Aos amigos, pela ajuda e claro pelas "baladas": Bárbara, Eliane e Daniel, Fábio, Glaúcio, Irene e Zé, Jaime, Natália S, Natália V, Raul, Regina, Said, Samuel, Sandra e Cristian. 
Finalmente, um agradecimento especial, merece o Prof. Michael Forger, pela excelente orientação. Além disso gostaria de agradecer a amizade e a disposição nestes anos de convívio.

Para todos aqueles que compartilharam esta passagem, e que nunca poderia citar aqui, deixo esta poesia

Levarás contigo

Meus olhos tão velhos?

Ah, deixa-os comigo

De que te servirão?

Levarás contigo

Minha boca e ouvidos?

Ah, deixa-os comigo

Degustei, ouvi

Tudo o que conheces

Coisas tão antigas.

Levarás contigo

Meu exalo nariz?

Ah, deixa-o comigo

Aspirou, torceu-se

Insignificante, mas meu.

E minha voz e cantiga?

Meu verso, meu dom

De poesia, sortilégio, vida?

Ah, leva-os contigo.

Por mim.

Hilda Hilst

Da morte. Odes Mínimas 


\title{
Resumo
}

Na generalização do arcabouço simplético da mecânica à teoria dos campos, o método tradicional baseado em dados iniciais não é covariante, pois depende da escolha "a priori" de uma hipersuperfície de Cauchy. Dois formalismos tem sido desenvolvidos para superar este problema: a abordagem multissimplética $[3,17,21,23,24,39]$ e a abordagem pelo espaço de fase covariante ou formalismo funcional covariante $[4,5,40,41]$. Uma questão central em ambas é a procura por uma definição natural de colchetes de Poisson. Na abordagem multissimplética, existem várias propostas de diferentes autores (veja, por exemplo, [12] e referências citadas), enquanto que na abordagem pelo espaço de fase covariante, a definição é originalmente devida a Peierls [32] e foi geometrizada por DeWitt [7-9]; a construção deste colchete de Peierls-DeWitt dentro do formalismo multissimplético foi recentemente elaborada por Forger e Romero [14,34]. Esta tese apresenta primeiros resultados mostrando que, pelo menos em certos casos especiais, os diferentes tipos de colchete de Poisson coincidem.

\begin{abstract}
In the generalization of the symplectic framework from mechanics to field theory, the traditional method based on initial data fails to be covariant because it depends on the "a priori" choice of a Cauchy hypersurface. Two formalisms have been developed to overcome this problem: the multisymplectic approach $[3,17,21,23,24,39]$ and the covariant phase space approach or covariant functional formalism $[4,5,40,41]$. A central issue in both of them is the quest for a natural definition of Poisson brackets. In the multisymplectic approach, there are various proposals by different authors (see, for example, [12] and references therein), while in the covariant phase space approach, the definition is originally due to Peierls [32] and has been geometrized by de Witt [7-9]; the construction of this Peierls - DeWitt bracket within the multisymplectic formalism has recently been worked out by Forger and Romero [14,34]. This thesis presents first results showing that, at least in certain special cases, the different types of Poisson brackets coincide.
\end{abstract}




\section{Conteúdo}

$\begin{array}{ll}\text { Introdução } & 1\end{array}$

1 Formalismo multissimplético $\quad 9$

1.1 Mecânica de sistemas não-autônomos . . . . . . . . . . . . . . . . . . . 11

1.2 Campos como seções de fibrados . . . . . . . . . . . . . . . . . 13

1.3 Fibrado dos jatos ........................... 16

1.3.1 Levantamento canônico . . . . . . . . . . . . . . . . . . 18

1.4 Fibrados de cojatos . . . . . . . . . . . . . . . . 20

1.4.1 Levantamento canônico . . . . . . . . . . . . . . . . . . 23

1.5 Geometria multissimplética . . . . . . . . . . . . . 27

1.5 .1 Espaços de multifase . . . . . . . . . . . . . . . . . 28

1.5.2 Levantamento hamiltoniano . . . . . . . . . . . . . . . . 33

1.6 Campos vetoriais hamiltonianos . . . . . . . . . . . . . . . . 38

1.6.1 Espaço de multifase estendido . . . . . . . . . . . . . . . . . 39

1.6.2 Espaço de multifase comum . . . . . . . . . . . . . 50

1.6.3 Projeção e levantamento de campos vetoriais hamiltonianos . . . . . 58

1.7 Colchete de Poisson . . . . . . . . . . . . . . . . . . . . . . 60 
2 Tensor de Poisson $\quad 65$

2.1 Conexões e derivadas covariantes . . . . . . . . . . . . . . . 66

2.2 Conexões lineares e duais lineares . . . . . . . . . . . . . . . . . . . 69

2.3 Conexões afins e duais afins . . . . . . . . . . . . . . . . 71

2.4 Conexões parciais . . . . . . . . . . . . . . . . . . . . 73

2.5 Conexões em fibrados compostos . . . . . . . . . . . . . . . . 77

2.6 Conexões no fibrado vertical . . . . . . . . . . . . . . . . . . 81

2.7 Conexões nos fibrados de jatos e cojatos . . . . . . . . . . . . . . 85

2.8 Referenciais de Darboux e tensor de Poisson . . . . . . . . . . . . . . . . 88

3 Formalismo funcional $\quad 95$

3.1 Espaço de fase covariante . . . . . . . . . . . . . . . . . . 95

3.2 Funcionais gerados por formas . . . . . . . . . . . . . . . . . . 100

3.3 Os teoremas principais . . . . . . . . . . . . . . . 103

A Referenciais não holônomos 109

A.1 Derivadas covariantes no fibrado vertical . . . . . . . . . . . . . . . . 109

A.2 Referenciais não holônomos no fibrado dos cojatos . . . . . . . . . . . . . . 112 


\section{Introdução}

Existe uma extensão natural, da mecânica para a teoria dos campos, do conceito do tensor de Poisson? Com esta pergunta iniciamos a pesquisa que deságua neste trabalho.

No início do século 19, ao estudar as equações de movimento na mecânica celeste, Poisson introduziu o colchete que leva hoje seu nome. Aproximadamente 30 anos depois, ao estudar as propriedades algébricas deste colchete, Jacobi descobriu sua famosa identidade e, na mesma época, Hamilton escreveu as equações de movimento da mecânica de sistemas conservativos na forma de um sistema de primeira ordem, hoje conhecido como as equações de Hamilton.

Uma novidade básica trazida por esta reformulação da mecânica clássica foi a introdução do conceito de espaço de fase de um sistema, como conjunto de todos os seus estados possíveis. Esta noção influenciou de forma fundamental o desenvolvimento de toda a física, principalmente em áreas como a mecânica estatística ou a mecânica quântica. Hoje, a sua implementação matemática correta ocupa uma posição central em qualquer teoria física.

Na própria mecânica clássica, o modelo matemático para espaços de fase passou por duas generalizações importantes. Originalmente, imaginou-se que o espaço de fase $P$ seria sempre derivado de um espaço de configuração $Q$, ou em termos matemáticos, existiria uma variedade $Q$ descrevendo as possíveis configurações do sistema tal que $P$ seria o fibrado cotangente $T^{*} Q$ de $Q$. No entanto, na década de 60 , as dificuldades de incorporar partículas com spin semi-inteiro levaram Souriau a propor um modelo matemático mais geral: o de uma variedade simplética. Finalmente, a partir da década de 70, começou a vingar um modelo ainda mais geral, protagonizado por Lichnerowicz e Weinstein, entre outros: o de uma variedade de Poisson. 
Uma variedade de Poisson é uma variedade $M$ munida de um colchete de Poisson, i.e., uma aplicação bilinear (sobre $\mathbb{R}$ ) e antisimétrica

$$
\{\cdot, \cdot\}: \mathcal{F}(M) \times \mathcal{F}(M) \longrightarrow \mathcal{F}(M)
$$

que satisfaz

i) a identidade de Jacobi:

$$
\{f,\{g, h\}\}+\{h,\{f, g\}\}+\{g,\{h, f\}\}=0
$$

ii) a regra de Leibniz:

$$
\{f, g h\}=\{f, g\} h+g\{f, h\} .
$$

Esta combinação de propriedades tem se revelado tão importante e útil que levou à definição de um novo tipo de álgebra: uma álgebra de Poisson é um espaço vetorial munido de duas composições internas - um produto associativo com unidade, comutativo ou não, e um colchete de Lie satisfazendo a regra de Leibniz como condição de compatibilidade. Além do seu caráter algébrico, a regra de Leibniz também tem um aspecto analítico e geométrico, pois afirma que para todo $f \in \mathcal{F}(M)$, a aplicação $g \mapsto\{f, g\}$ é uma derivação em $\mathcal{F}(M)$ e portanto existe um campo vetorial $X_{f}$ em $M$ tal que $X_{f}(g)=\{f, g\}$. Em particular, se a função $f$ for a Hamiltoniana $H$, o fluxo do campo vetorial $X_{H}$ correspondente descreve a evolução temporal do sistema.

O mesmo argumento pode ser aplicado aos dois argumentos do colchete de Poisson simultaneamente: como este é antisimétrico e é uma derivação em cada um dos seus argumentos, existe um único campo bivetorial $\pi$ sobre $P$, chamado o tensor de Poisson, tal que, para quaisquer duas funções $f, g \in \mathcal{F}(P)$, vale

$$
\{f, g\}=\pi(d f \wedge d g) .
$$

Reinterpretando $\pi$ como homomorfismo de fibrados vetoriais

$$
\pi: T^{*} P \longrightarrow T P
$$

temos simplesmente

$$
X_{f}=\pi(d f) .
$$

A identidade de Jacobi para o colchete de Poisson pode então ser reformulada como a. condição de que o colchete de $\operatorname{Schouten}^{1}$ de $\pi \operatorname{com} \pi$ mesmo se anule:

$$
[\pi, \pi]=0 .
$$

\footnotetext{
${ }^{1}$ Uma definição explícita do colchete de Schouten para campos multivetoriais é dada na Seção 1.7.
} 
Uma classe especial de variedades de Poisson são as variedades simpléticas. Uma variedade simplética é uma variedade munida de uma dois-forma $\omega$ fechada $(d \omega=0)$ e nãodegenerada. Esta forma chama-se forma simplética e o tensor de Poisson obtido a partir desta estrutura simplética é não-degenerado. A não-degenerescência de $\omega$ fornece um meio de definirmos, de maneira única, para cada função um campo vetorial; dada uma função $f$ pode-se associar à 1 -forma $d f$ o campo vetorial $X_{f}$ dado por

$$
i_{X_{f}} \omega=d f .
$$

Neste caso o colchete é expresso pela fórmula

$$
\{f, g\}=i_{X_{g}} i_{X_{f}} \omega .
$$

Obviamente, o colchete assim definido é antisimétrico, e mostra-se que ele satisfaz a identidade de Jacobi se e somente se $\omega$ for fechada.

Dentro da classe das variedades simpléticas, destacam-se os fibrados cotangentes. O fibrado cotangente $T^{*} Q$ de uma variedade $Q$ qualquer, com projeção $\tau_{Q}^{*}$, vem munido de uma um-forma $\theta$ dada por

$$
\theta(z)(v)=z\left(T_{z} \tau_{Q}^{*} \cdot v\right),
$$

onde $z \in T^{*} Q$ e $v \in T_{z}\left(T^{*} Q\right)$. Esta forma chama-se forma canônica, e sua derivada exterior (a menos de sinal) é a forma simplética:

$$
\omega=-d \theta
$$

Na modelagem matemática do conceito de espaço de fase, existem dois aspectos importantes que motivaram a passagem de fibrados cotangentes para variedades simpléticas. Um deles foi a observação, devida a Souriau, que isso permite incluir, de forma natural, o conceito de spin na mecânica clássica, mesmo quando este for semi-inteiro, usando a esfera $S^{2}$ como a parte do espaço de fase que descreve este grau de liberdade. O outro encontra-se no fato de que variedades simpléticas constituem um âmbito matemático natural para o processo de redução do espaço de fase, conforme descrito pelo teorema de Marsden e Weinstein. Exemplos importantes de variedades simpléticas que, tipicamente, não são fibrados cotangentes incluem as órbitas coadjuntas de grupos de Lie ou variedades de Kähler.

De forma semelhante, existem variedades de Poisson que não são simpléticas, o que ocorre quando o tensor de Poisson não tiver posto máximo, podendo inclusive deixar de ter posto constante. O exemplo mais simples é o espaço dual $\mathfrak{g}^{*}$ de uma álgebra de Lie $\mathfrak{g}$. Uma outra classe de exemplos é formada pelos grupos de Lie-Poisson, uma vez que um grupo de Lie semisimples não possui tensores de Poisson invariantes que sejam não-degenerados (ou seja, não existem grupos de Lie simpléticos semisimples). Um teorema geral de Weinstein estabelece que qualquer variedade de Poisson admite uma decomposição canônica em 
subvariedades simpléticas, na forma de uma folheação (singular). O exemplo mais simples é a decomposição do espaço dual $\mathrm{g}^{*}$ de uma álgebra de Lie g nas órbitas coadjuntas, em particular a de $\mathbb{R}^{3}$ em esferas: a menor órbita $\{0\}$ consiste de um único ponto, enquanto que as órbitas de maior dimensão são as órbitas regulares.

Passando da mecânica clássica à teoria clássica dos campos, o método mais tradicional de implementar o formalismo canônico é baseado na identificação das variáveis dinâmicas da teoria como dados iniciais, ou seja, funções sobre alguma hipersuperfície de Cauchy no espaço-tempo, sendo que o formalismo providencia equações diferenciais para a evolução temporal fora desta hipersuperfície. O problema principal desta abordagem reside no fato de que pela mera escolha de uma hipersuperfície de Cauchy específica para carregar os dados iniciais, perde-se a covariância explícita da teoria, isto é, a invariância de Lorentz (no contexto da relatividade restrita) e mais geralmente a invariância sob transformações gerais de coordenadas do espaço-tempo (no contexto da relatividade geral). Isso não significa que estas simetrias fundamentais sejam quebradas, mas elas (ou pelo menos uma parte delas) deixam de se manifestar de forma direta: podemos dizer que são ofuscadas. No entanto, isso pode criar problemas muito mais sérios do que uma mera falta de elegância quando queremos aplicar algum método de quantização, pois nada garante a priori que este método seja compatível com tais "simetrias escondidas". De fato, quantização canônica conduz a modelos da teoria quântica dos campos cuja covariância está longe de ser óbvia e de fato constitui um problema formidável: como um exemplo bem conhecido, podemos citar os esforços necessários para verificar a invariância de Lorentz da eletrodinâmica quântica (perturbativa) no calibre de Coulomb.

Estas e outras observaçọes similares têm por muitas décadas motivado tentativas de desenvolver, na teoria clássica dos campos, um formalismo canônico inteiramente covariante, que poderia servir como ponto inicial para métodos alternativos de quantização. Entre muitas idéias que foram propostas nesta direção, duas vieram ocupar um papel de destaque. A primeira é o "formalismo funcional covariante", baseado no conceito do "espaço de fase covariante " que é definido como o espaço das soluções das equações de movimento, ao invés de um espaço de dados de Cauchy para estas equações. Esta formulação foi popularizada nos 1980's por Crnković, Witten e Zuckerman [4,5,41] (veja também [40]) que mostraram como construir, para alguns dos modelos mais importantes da teoria dos campos (incluíndo teorias de calibre e relatividade geral), uma estrutura simplética no espaço de fase covariante. A segunda tornou-se conhecida como o "formalismo multissimplético", baseado no conceito do "espaço de multifase" que, basicamente, pode ser definido localmente associando a cada coordenada $q^{i}$ não apenas um momento conjugado $p_{i}$ mas $n$ momentos conjugados $p_{i}^{\mu}$ $(\mu=1, \ldots, n)$, onde $n$ é a dimensão da variedade espaço-tempo subjacente. Em coordenadas locais, esta construção recorre aos trabalhos clássicos de De Donder e Weyl nos 1930's [6,39]. No entanto, a formulação global é muito mais recente: foi iniciada nos 1970's por um grupo de físicos matemáticos, principalmente na Polônia [21,23,24] mas também em 
outras partes do mundo [15,18], e foi definitivamente estabelecida nos 1990's [3, 16]; uma exposição detalhada, com vários exemplos, pode ser encontrada no "GIMmsy paper" [17].

Os dois formalismos, embora inteiramente covariantes e dirigidos para o mesmo objetivo último, são completamente diferentes na natureza; cada um deles tem seus próprios méritos e inconvenientes.

- O formalismo multissimplético é manifestamente consistente com os princípios básicos da teoria dos campos, em particular o de localidade, e é matematicamente rigoroso pois usa apenas métodos bem estabelecidos do cálculo em variedades de dimensão finita. Por outro lado, não parece permitir nenhuma definição óbvia do colchete de Poisson entre observáveis. De fato, a proposta original de simplesmente adotar a mesma fórmula (9) da mecânica cria um problema sério, a saber que a identidade de Jacobi deixa de ser válida. Este defeito foi recentemente corrigido modificando a definição pela adição de um termo exato $[11,12]$, mas várias outras dificuldades não foram superadas, entre elas a aparente inexistência de um produto associativo com unidade em relação ao qual este colchete de Poisson satisfaria uma regra de Leibniz. Ademais, a introdução de $n$ momentos conjugados a cada coordenada obscurece a dualidade usual entre variáveis canonicamente conjugadas (tais como momentos e posições), que desempenha um papel fundamental em todos os métodos conhecidos de quantização. Uma solução definitiva destes problemas ainda precisa ser encontrada.

- O formalismo funcional covariante está em concordância com os princípios que regem a geometria simplética; em particular, ele admite uma definição natural do colchete de Poisson, devida a Peierls [32] e elaborada posteriormente por DeWitt [7-9], que preserva a dualidade entre variáveis canonicamente conjugadas. Por outro lado, sendo de natureza intrinsecamente global, tal abordagem obscurece a implementação do princípio de localidade. Além disso, este formalismo não pode ser considerado matematicamente rigoroso, uma vez que está essencialmente restrito à aplicação formal de métodos do cálculo em variedades, extrapolados ao caso de dimensão infinita: transformar os resultados formais assim obtidos em teoremas matemáticos é um problema separado, frequentemente bem complexo e difícil.

A relação exata entre as duas formulações vem sendo buscada desde os 1970's mas ainda está longe de ser completamente entendida. Avanços significativos tem sido alcançados nos últimos anos e o presente trabalho pretende contribuir nesta direção.

Os resultados principais desta tese encontram-se no Capítulo 3; são os Teoremas 3.2 e 3.3. O segundo e o último deles estabelecem uma estreita relação entre o colchete de Poisson do formalismo multissimplético $[11,12]$ entre formas diferenciais no espaço de multifase comum e o colchete de Peierls-DeWitt entre funcionais definidos a partir de tais formas, para o caso 
especial em que as referidas formas são formas hamiltonianas de grau $n-1$ e os funcionais correspondentes são obtidos, essencialmente, por pull-back destas formas com uma solução das equações de movimento do modelo e subsequente integração sobre uma subvariedade de codimensão 1 do espaço-tempo, tipicamente uma hipersuperfície de Cauchy.

Outro aspecto do nosso trabalho é a tentativa de identificar, dentro do formalismo multissimplético, um objeto análogo ao tensor de Poisson do formalismo simplético. No contexto da mecânica, o tensor de Poisson $\pi$ é o dual da forma simplética $\omega$, o que em coordenadas locais de Darboux $\left(q^{i}, p_{i}\right)$ pode ser representado pela fórmula

$$
\omega=d q^{i} \wedge d p_{i}, \pi=\frac{\partial}{\partial q^{i}} \wedge \frac{\partial}{\partial p_{i}} .
$$

Por outro lado, a relação entre o tensor de Poisson e o colchete de Poisson entre funções sobre o espaço de fase é estabelecida pela equação (4) que, quando escrita em coordenadas locais de Darboux $\left(q^{i}, p_{i}\right)$, leva à fórmula padrão

$$
\{f, g\}=\frac{\partial f}{\partial q^{i}} \frac{\partial g}{\partial p_{i}}-\frac{\partial g}{\partial q^{i}} \frac{\partial f}{\partial p_{i}} .
$$

Afirmações análogas, pelo menos em nível formal, valem para o formalismo funcional covariante da teoria dos campos: Aqui, a forma simplética funcional $\Omega$ é dada pela integral da "corrente simplética" de Crnković, Witten e Zuckerman [4,5,41] (veja também [40]) sobre uma hipersuperfície de Cauchy $\Sigma$ no espaço-tempo $M$, enquanto que o colchete de PeierlsDeWitt, que segundo o resultado principal de [14,34] é o colchete de Poisson correspondente, conforme o análogo funcional das equações (8) e (9), é dado por uma integral dupla envolvendo a função de Green causal do operador linearizado. Isso pode ser interpretado como dizendo que o tensor de Poisson funcional $\Pi$ correspondente, conforme o análogo funcional da equação (4), é um operador integral em $M \times M$ cujo núcleo é a referida função de Green causal.

No caso do formalismo multissimplético, uma possível proposta para um tensor de Poisson consistiria em introduzir, como dual da forma multissimplética $\omega$, que é de grau $n+1$, um campo multivetorial $\pi$, também de grau $n+1$, tal que em coordenadas locais adaptadas onde

$$
\omega=d q^{i} \wedge d p_{i}^{\mu} \wedge d^{n} x_{\mu}-d p \wedge d^{n} x
$$

teríamos

$$
\pi=\frac{\partial}{\partial q^{i}} \wedge \frac{\partial}{\partial p_{i}^{\mu}} \wedge \frac{\partial^{n}}{\partial x_{\mu}}-\frac{\partial}{\partial p} \wedge \frac{\partial^{n}}{\partial x} .
$$


Entretanto, ao contrário de $\omega$, este objeto não é um campo tensorial, pois não se transforma de maneira natural sob mudança de coordenadas. Outro problema é que a definição ingênua de um colchete de Poisson a partir de uma expressão análoga à da mecânica clássica, tal que

$$
\{f, g\}=i_{\pi}(d f \wedge d g)
$$

é insatisfatória.

Como será mostrado no Capítulo 2 desta tese, o primeiro problema pode ser resolvido pela introdução de um referêncial apropriado, geralmente não holônomo, que chamaremos de referêncial de Darboux e que pode ser definido a partir de um conjunto apropriado de conexões $\Gamma$, em relação ao qual

$$
\omega=e^{i} \wedge e_{i}^{\mu} \wedge e_{\mu}^{n}-e_{0} \wedge e^{n}
$$

$\mathrm{e}$

$$
\pi_{\Gamma}=e_{i} \wedge e_{\mu}^{i} \wedge e_{\mu}^{n}-e^{0} \wedge e_{n} .
$$

Mais exatamente, provamos que o campo multivetorial $\pi_{\Gamma}$ é completamente determinado em termos de

- uma conexão geral no espaço de configuração $E$,

- uma conexão linear parcial no fibrado vertical $V E$ de $E$ ao longo de $V E$,

- uma conexão linear na variedade base $M$.

No entanto, é claro que a introdução de tais conexões configura uma estrutura adicional cuja interpretação física permanece obscura. Portanto, o resultado desta investigação é uma afirmação negativa: não parece existir um conceito natural de tensor de Poisson na geometria multissimplética. 


\section{Formalismo multissimplético}

Durante os últimos anos, evidenciou-se cada vez mais que o âmbito matemático adequado para a formulação geométrica da teoria clássica dos campos é o que pode ser chamado de geometria multissimplética. Esta extensão da geometria simplética, que na física corresponde à passagem da mecânica à teoria dos campos, teve início nos trabalhos de Cartan, De Donder e Weyl. A idéia central deste formalismo é tratar todas as variáveis espaço-temporais da teoria dos campos (e não apenas a variável temporal) da mesma maneira que a variável temporal da mecânica. Assim, em um sistema com $N$ graus internos de liberdade sobre um espaçotempo $n$-dimensional $M$, teremos para cada campo clássico $\varphi^{i}(i=1, \ldots, N)$ não somente um campo conjugado mas $n$ campos conjugados $\pi_{i}^{\mu}(i=1, \ldots, N, \mu=1, \ldots, n)$. No formalismo lagrangiano, onde a dinâmica de um modelo é determinada pela densidade lagrangiana $L\left(x^{\mu}, \varphi^{i}, \partial_{\mu} \varphi^{i}\right)$, estes campos conjugados são obtidos pela correspondente transformação de Legendre, i.e.,

$$
\pi_{i}^{\mu}=\frac{\partial L}{\partial \partial_{\mu} \varphi^{i}}
$$

e a hamiltoniana covariante ou hamiltoniana de De Donder-Weyl é

$$
H\left(x^{\mu}, \varphi^{i}, \pi_{i}^{\mu}\right)=\pi_{i}^{\mu} \partial_{\mu} \varphi^{i}-L
$$

No formalismo lagrangiano, as equações do movimento são as equações de Euler -Lagrange

$$
\partial_{\mu}\left(\frac{\partial L}{\partial \partial_{\mu} \varphi^{i}}\right)-\frac{\partial L}{\partial \varphi^{i}}=0
$$

Sob passagem ao formalismo hamiltoniano, obtemos como equações de movimento as equações de De Donder-Weyl

$$
\frac{\partial H}{\partial \pi_{i}^{\mu}}=\partial_{\mu} \varphi^{i} \quad, \quad \frac{\partial H}{\partial \varphi^{i}}=-\partial_{\mu} \pi_{i}^{\mu} .
$$


Mediante condições de regularidade para $L$ e/ou $H$, essas duas versões de equações de movimento são equivalentes.

Observemos a similaridade com a situação encontrada na mecânica. De fato, veremos na primeira seção que, quando $n=1$, recuperamos a mecânica (não-autonoma). A principal novidade reside no fato de que, em geral, temos $n$ multimomenta $\pi_{i}^{\mu}$ associados a cada grau de liberdade $\varphi^{i}$. Outras diferenças estão na natureza das variáveis, tanto das independentes como das dependentes: Na mecânica usa-se simplesmente uma cópia da reta real $\mathbb{R}$ para representar o tempo, enquanto que na teoria dos campos o espaço-tempo é modelado por uma variedade $M$ que nem sempre será trivial. Na mecânica, as variáveis dinâmicas são representadas por curvas em uma variedade "interna" $Q$, chamada de espaço de configuração, enquanto que, como explicamos com mais detalhes na segunda seção, na teoria dos campos elas são representadas por seções de um fibrado $E$ sobre $M$ que também nem sempre será trivial (é da forma $M \times Q$ apenas localmente). Para acomodar derivadas, o papel análogo ao do fibrado tangente $T Q$ de $Q$ na mecânica é desempenhado pelo fibrado dos jatos $J E$ (de primeira ordem) de $E$ [35]. De forma semelhante, o papel análogo ao do fibrado cotangente $T^{*} Q$ de $Q$ na mecânica é desempenhado por um dos duais de $J E$, mais exatamente o dual afim torcido $J^{\circledast} E$ de $J E$. Além destes, o formalismo também usa o fibrado dos jatos linearizado $\vec{J} E$ de $E$ e seu dual linear torcido $\vec{J}^{\circledR} E$. A construção destes objetos será apresentada. na terceira e quarta seção. Também incluímos uma breve exposição do processo de levantamento canônico de automorfismos (infinitesimais) do fibrado $E$ sobre $M$ para automorfismos (infinitesimais) do fibrado dos jatos $J E$ e dos seus vários duais, assim como sua ação sobre seções destes fibrados. Na quinta seção, discutimos a construção da forma multicanônica $\theta$ e da forma multissimplética $\omega$ sobre $J^{\circledast} E$ e introduzimos a forma multicanônica $\theta_{\mathcal{H}}$ e a forma multissimplética $\omega_{\mathcal{H}}$ sobre $\vec{J}^{\circledast} E$ associadas a uma hamiltoniana $\mathcal{H}$, que é uma seção de $J^{\circledast} E$ visto como fibrado afim em linhas sobre $\vec{J}^{\circledR} E$, obtidas simplesmente por pull-back [3]. Também abordamos as relações entre $\theta, \omega$ e o campo de Euler $\Sigma$ sobre $J^{\otimes} E$ que existe em função de $J^{\oplus} E$ ser o espaço total de um fibrado vetorial sobre $E$. Assim, verifica-se porque o dual afim torcido $J^{\circledast} E$ de $J E$ é o espaço de multifase estendido e o dual linear torcido $\vec{J}^{\circledR} E$ é o espaço de multifase comum da geometria multissimplética. Na sexta seção, introduzimos campos vetoriais localmente hamiltonianos e exatamente hamiltonianos sobre $J^{\otimes} E$ assim como sobre $\vec{J}^{\circledast} E$ e apresentamos resultados sobre sua estrutura que, no primeiro caso, foram obtidos recentemente em [13]. Na sétima seção, recordamos o conceito de $(n-1)$-formas hamiltonianas e a definição do colchete de Poisson de tais formas segundo a filosofia geral adotada em [11-13,20]. Estes resultados serão importantes para a demonstração do teorema principal desta tese, que está no Capítulo 3. 


\subsection{Mecânica de sistemas não-autônomos}

Todas as formulações usuais da mecânica clássica baseiam-se no preceito de tratar a variável independente, o tempo, como um parâmetro externo de evolução. Aspectos geométricos que levam à introdução de variedades não-triviais, seja como modelo para o espaço de configuração $Q$, seja como modelo para o espaço de fase $P$, não afetam a variável tempo $t$ que parametriza as curvas em $Q$ ou $P$ representando as soluções das equações de movimento. Claramente, esta independência de $t$ das variáveis dinâmicas do sistema é uma expressão do axioma do tempo absoluto de Newton. No entanto, sabemos que este ponto de vista não poderá ser mantido quando queremos incorporar o princípio da relatividade - seja restrita, seja geral. Portanto, faz-se necessário, como primeiro passo, adaptar o formalismo de tal forma a colocar a variável tempo em evidência.

A inclusão da variável tempo dentro do formalismo geral da mecânica torna-se imprescindível, mesmo no âmbito da mecânica clássica newtoniana, para a descrição de sistemas não-autônomos, tais como a mecânica de partículas ou sistemas de partículas em campos externos que dependem explicitamente do tempo. O procedimento padrão para tratar desta situação no contexto lagrangiano consiste em substituir o espaço de configuração $Q$, com coordenadas locais típicas $q^{i}$, pelo produto cartesiano $\mathbb{R} \times Q$, com coordenadas locais típicas $\left(t, q^{i}\right)$, e o espaço de velocidades $T Q$, com coordenadas locais típicas $\left(q^{i}, \dot{q}^{i}\right)$, pelo produto cartesiano $\mathbb{R} \times T Q$, com coordenadas locais típicas $\left(t, q^{i}, \dot{q}^{i}\right)$, permitindo assim a inclusão de lagrangianas $L$ que dependem explicitamente do tempo $\left(L=L\left(t, q^{i}, \dot{q}^{i}\right)\right)$. De maneira análoga, no contexto hamiltoniano devemos substituir o espaço de fase $P$, com coordenadas locais típicas $\left(q^{i}, p_{i}\right)$, pelo produto cartesiano $\mathbb{R} \times P$, com coordenadas locais típicas $\left(t, q^{i}, p_{i}\right)$, permitindo assim a inclusão de hamiltonianas $H$ que dependem explicitamente do tempo $\left(H=H\left(t, q^{i}, p_{i}\right)\right)$. Globalmente, se $P$ for uma variedade simplética, $\mathbb{R} \times P$ vem munido de uma dois-forma fechada que é simplesmente o pull-back da forma simplética de $P$ pela projeção sobre o segundo fator; no entanto, esta dois-forma é degenerada, com núcleo que forma uma distribuição involutiva uni-dimensional. Este tipo de estrutura tem sido axiomatizado sob o título de "variedades de contato". Como veremos ela admite uma extensão natural à teoria dos campos, porém não-degenerada.

A solução do problema de definir uma versão estendida do conceito de espaço de fase que inclua a variável tempo mas sem alterar o tipo de modelo matemático empregado já foi claramente enunciada por Cartan. Ao invés do "espaço de fase simplesmente estendido" $\mathbb{R} \times P$, com coordenadas locais típicas $\left(t, q^{i}, p_{i}\right)$, considere o "espaço de fase duplamente estendido" $\mathbb{R} \times P \times \mathbb{R}$, com coordenadas locais típicas $\left(t, q^{i}, p_{i}, p\right)$, onde $p$ é uma variável com a dimensão física de uma energia. (Usamos o símbolo $p$, ao invés de $E$, para evitar uma mudança de notação na hora da passagem da mecânica para a teoria dos campos.) Obviamente, como $\mathbb{R}^{2}$ é o fibrado cotangente de $\mathbb{R}, \mathbb{R} \times P \times \mathbb{R}$ será uma variedade do mesmo tipo que $P$ : se $P=T^{*} Q$ com forma canônica dada por $\theta_{P}=p_{i} d q^{i}$, então 
$\mathbb{R} \times P \times \mathbb{R}=T^{*}(\mathbb{R} \times Q)$ com forma canônica dada por

$$
\theta=p_{i} d q^{i}+p d t
$$

enquanto que se $P$ for variedade simplética com forma simplética dada por $\omega_{P}=d q^{i} \wedge d p_{i}$, então $\mathbb{R} \times P \times \mathbb{R}$ também será variedade simplética, com forma simplética dada por

$$
\omega=d q^{i} \wedge d p_{i}-d p \wedge d t
$$

e finalmente se $P$ for variedade de Poisson com tensor de Poisson $\pi_{P}, \mathbb{R} \times P \times \mathbb{R}$ também sera variedade de Poisson, com tensor de Poisson dado por

$$
\pi=\pi_{P}-\frac{\partial}{\partial p} \wedge \frac{\partial}{\partial t} .
$$

Uma outra vantagem de incluir a variável tempo nos diversos espaços que descrevem as variáveis dinâmicas é que este procedimento permite abordar o conceito das "simetrias locais" que estão no coração das modernas teorias de calibre, de suma importância na formulação de modelos para as interações fundamentais na física das partículas. Em geral, simetrias contínuas são descritas por algum grupo de Lie $G$ em conjunto com uma ação deste grupo sobre o espaço de variáveis dinâmicas pertinente. A formulação padrão da mecânica permite considerar apenas "simetrias globais", onde as transformações de simetria são independentes do tempo. A inclusão de "simetrias locais" requer permitir que as transformações de simetria possam depender explicitamente do tempo; tais simetrias locais são geralmente conhecidas como "transformações de calibre". Em termos geométricos, isto significa considerar os espaços estendidos $\mathbb{R} \times Q, \mathbb{R} \times T Q, \mathbb{R} \times P, \mathbb{R} \times P \times \mathbb{R}$ etc. como fibrados sobre a reta real que representa o tempo, abrindo assim a possibilidade de interpretar transformações de calibre como mudanças de trivialização nestes fibrados. Observa-se que segundo um teorema geral de topologia, todos os fibrados sobre $\mathbb{R}$ são triviais, mas vale salientar que não existe em geral nenhuma trivialização preferida, e portanto o "princípio de invariância de calibre", ou seja, o princípio de invariância sob mudança de trivialização, continua sendo de grande importância mesmo nesta situação.

Como exemplo, podemos notar que aplicando o princípio de invariância de calibre a qualquer um dos espaços estendidos acima mencionados elimina o status privilegiado da segunda projeção. Em particular, isso significa que a projeção de $\mathbb{R} \times P$ sobre $P$ usada na definição da estrutura de contato sobre $\mathbb{R} \times P$ não é invariante de calibre. Este fato é a origem das dificuldades encontradas em tentativas de formular a mecânica hamiltoniana de partículas acopladas a campos eletromagnéticos e/ou de Yang-Mills usando o formalismo de variedades de contato.

A reinterpretação de $\mathbb{R} \times Q$ como fibrado sobre $\mathbb{R}$ acarreta uma série de consequências não-triviais. Primeiro, permite concluir que $\mathbb{R} \times T Q$ é na verdade o fibrado dos jatos (de 
primeira ordem) de $\mathbb{R} \times Q$. Como tal, este é novamente um fibrado sobre $\mathbb{R}$ mas também é um fibrado sobre $\mathbb{R} \times Q$, e mais precisamente, um fibrado afim. Podemos perguntar por que não seria um fibrado vetorial, já que $T Q$ é um fibrado vetorial sobre $Q$. A razão da perda da estrutura linear é justamente a possibilidade de efetuar transformações de calibre, ou seja, transformações de coordenadas locais na variedade $Q$ que podem depender do tempo. Neste caso, a lei de transformação para as coordenadas locais induzidas em $T Q$ adquire, além do termo linear envolvendo a jacobiana, um termo constante envolvendo a derivada temporal. Os espaços de fase duplamente estendido $\mathbb{R} \times P \times \mathbb{R}$ e simplesmente estendido $\mathbb{R} \times P$ podem então ser definidos como, respectivamente, o dual afim do fibrado dos jatos e o dual linear do fibrado dos jatos linearizado, sendo o primeiro um fibrado afim em linhas sobre o segundo. Como resultado, é possível considerar a hamiltoniana de um sistema mecânico como uma seção deste fibrado $(\mathbb{R} \times P \times \mathbb{R} \longrightarrow \mathbb{R} \times P)$ : em coordenadas locais, é definida por $\left(t, q^{i}, p_{i}\right) \mapsto\left(t, q^{i}, p_{i}, p\right) \operatorname{com}^{1}$

$$
p=-H\left(t, q^{i}, p_{i}\right) .
$$

Ademais, soluções das equações de movimento tornam-se seções dos fibrados $\mathbb{R} \times Q$ (no caso lagrangiano) ou $\mathbb{R} \times P$ (no caso hamiltoniano) sobre $\mathbb{R}$ : em coordenadas locais, são dadas por $t \mapsto\left(t, q^{i}(t)\right)$ ou $t \mapsto\left(t, q^{i}(t), p_{i}(t)\right)$, respectivamente.

Uma descrição mais detalhada destas construções, num contexto bem mais geral, será apresentada na próxima seção. Por enquanto, mencionamos apenas que as formas $\theta$ e $\omega$, assim como o tensor de Poisson $\pi$, dados em coordenadas locais pelas equações (1.5), (1.6) e (1.7), possuem um significado global, ou seja, independente da escolha de um sistema de coordenadas, mesmo quando admitirmos transformações de coordenadas $q^{i} \rightarrow q^{\prime k}$ que dependem do tempo. Esta invariância adicional é altamente não-trivial e não pode ser deduzida de nenhum dos argumentos apresentados anteriormente, mas ela pode ser verificada ou por cálculo explícito ou como resultado das construções globais a serem discutidas na próxima seção.

\subsection{Campos como seções de fibrados}

O ponto inicial para a formulação geométrica da teoria clássica dos campos é a escolha de um fibrado de configuração, que em general pode ser um fibrado sobre um espaço-tempo cujas seções sejam os campos da teoria sob consideração. No que segue, denotaremos seu espaço total por $E$, seu espaço base por $M$, sua fibra típica por $Q$ e a projeção de $E$ sobre $M$ por $\pi$; as dimensões são

$$
\operatorname{dim} M=n, \operatorname{dim} Q=N, \operatorname{dim} E=n+N .
$$

\footnotetext{
${ }^{1} \mathrm{O}$ sinal nesta equação é meramente convencional.
} 
De forma geral, $M$ é interpretado como espaço-tempo enquanto $Q$ é o espaço de configuração da teoria - uma variedade cujas coordenadas (locais) descrevem graus internos de liberdade. ${ }^{2}$ O espaço total $E$ é localmente mas não necessariamente globalmente isomorfo ao produto cartesiano $M \times Q$, mas deve-se salientar que mesmo quando o fibrado de configuração for globalmente trivial, não existe em geral nenhuma trivialização preferida, e é precisamente a liberdade de mudar de trivialização que permite incorporar as teorias do calibre neste contexto. Um outro ponto que merece ser enfatizado é que o fibrado de configuração em geral não possui nenhuma estrutura adicional: estas aparecem somente quando nos focalizamos em classes especiais de modelos.

- Fibrados vetoriais surgem naturalmente nas teorias com campos lineares de matéria e também na relatividade geral: o tensor métrico é um exemplo.

- Fibrados afins podem ser empregados para acomodar campos de calibre, uma vez que conexões principais em um fibrado principal $P$ sobre o espaço-tempo $M$ podem ser vistas como seções do fibrado das conexões de $P$ - um fibrado afim $C P$ sobre $M$ construído a partir de $P$.

- Fibrados gerais são empregados para acomodar campos não-lineares de matéria, em particular aqueles que correspondem à aplicações do espaço-tempo $M$ para alguma variedade alvo $Q$ : um exemplo padrão são os modelos sigma não-lineares.

Para cobrir toda esta variedade de situações, as construções gerais nas quais se baseia a formulação geométrica da teoria clássica dos campos não devem depender da escolha de qualquer estrutura adicional no fibrado de configuração. Esta exigência é satisfeita naturalmente no formalismo do espaço de multifase - ao contrário de muitas propostas que se encontram na literatura: na sua maioria, estas dependem da escolha a priori de uma conexão no fibrado de configuração, excluindo assim as teorias de calibre nas quais conexões devem ser tratadas como variáveis dinâmicas e não como campos de fundo fixos.

Neste formalismo, simetrias são realizadas por automorfismos do fibrado $E$ sobre $M$ e geradores de grupos a um parâmetro de simetrias por campos vetoriais projetáveis sobre $E$.

Um automorfismo de um fibrado geral $E$ sobre $M$ com projeção $\pi$ é um difeomorfismo $\Phi_{E}: E \rightarrow E$ que preserva fibras. Isto implica que $\Phi_{E}$ induz um difeomorfismo $\Phi_{M}: M \rightarrow M$ tal que o diagrama

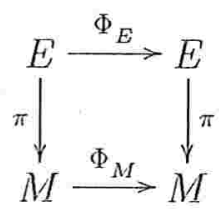

\footnotetext{
${ }^{2}$ Esta interpretação muda na teoria das cordas e das membranas.
} 
é comutativo; dizemos que $\Phi_{E}$ é um automorfismo de fibrados sobre $\Phi_{M}$. Como caso especial, um automorfismo $\Phi_{E}$ de $E$ é chamado estrito se $\Phi_{M}$ for a identidade. Podemos introduzir ainda o suporte de um difeomorfismo $\Phi_{M}$ de $M$ como sendo o fecho do conjunto de pontos em $M$ sobre os quais ele não age como a identidade,

$$
\operatorname{supp} \Phi_{M}=\overline{\left\{x \in M / \Phi_{M}(x) \neq x\right\}},
$$

e de forma similar, o suporte na base de um automorfismo $\Phi_{E}$ de $E$ como sendo o fecho do conjunto de pontos de $M$ para os quais ele não age como a identidade na fibra respectiva,

$$
\operatorname{supp} \Phi_{E}=\overline{\left\{x \in M / \Phi_{E}(e) \neq e \text { para algum } e \in E_{x}\right\}} .
$$

Passando a campos vetoriais como geradores de grupos a um parâmetro de difeomorfismos, através do seu fluxo, diremos que um campo vetorial $X_{E}$ sobre $E$ é projetável se vale

$$
T_{e_{1}} \pi\left(X_{E}\left(e_{1}\right)\right)=T_{e_{2}} \pi\left(X_{E}\left(e_{2}\right)\right) \quad \text { para } e_{1}, e_{2} \in E \operatorname{com} \pi\left(e_{1}\right)=\pi\left(e_{2}\right),
$$

onde $T \pi: T E \rightarrow T M$ é a aplicação tangente à projeção $\pi: E \rightarrow M$. Isto implica que $X_{E}$ induz um campo vetorial $X_{M}$ sobre $M$ ao qual é $\pi$-relacionado:

$$
X_{M}(x)=T_{e} \pi\left(X_{E}(e)\right) \quad \text { para } x \in M \text { e } e \in E \operatorname{com} \pi(e)=x .
$$

Também diremos que $X_{E}$ recobre $X_{M}$. Como caso especial, um campo vetorial projetável $X_{E}$ sobre $E$ é chamado vertical se $X_{M}$ for zero. Às vezes, é conveniente pensar em um automorfismo $\Phi$ de um fibrado como o par $\left(\Phi_{E}, \Phi_{M}\right)$ e em um campo vetorial projetável $X$ sobre um fibrado como o par $\left(X_{E}, X_{M}\right)$. Finalmente, cabe mencionar que um termo alternativo para "campo vetorial projetável" é "automorfismo infinitesimal de fibrado".

Um ponto importante para este trabalho é entender como automorfismos e automorfismos infinitesimais de fibrados agem sobre suas seções. Primeiro, a ação de um automorfismo $\Phi=\left(\Phi_{E}, \Phi_{M}\right)$ sobre uma seção $\varphi$ de $E$ fornece uma nova seção $\Phi \cdot \varphi$ de $E$, dada por:

$$
(\Phi \cdot \varphi)(x)=\Phi_{E} \cdot\left(\varphi\left(\Phi_{M}^{-1}(x)\right)\right) \text { para } x \in M .
$$

Segundo, a ação de um automorfismo infinitesimal $X=\left(X_{E}, X_{M}\right)$ sobre uma seção $\varphi$ de $E$ fornece uma seção $\delta_{X} \varphi$ do fibrado vetorial $\varphi^{*}(V E)$ sobre $M$ que é o pull-back do fibrado vertical $V E$ de $E$ via $\varphi$, obtida da fórmula anterior por diferenciação em relação ao parâmetro de fluxo:

$$
\delta_{X} \varphi(x)=X_{E}(\varphi(x))-T_{x} \varphi \cdot X_{M}(x) \text { para } x \in M .
$$

Em coordenadas locais adaptadas, constituídas por coordenadas locais $x^{\mu}$ para a variedade base $M$, coordenadas locais $q^{i}$ para a fibra típica $Q$ e uma trivialização local de $E$ sobre $M$, temos

$$
\delta_{X} \varphi^{i}=X^{i}(\varphi)-\partial_{\mu} \varphi^{i} X^{\mu}
$$




\subsection{Fibrado dos jatos}

A construção do fibrado dos jatos associado a um fibrado geral $E$ é padrão e pode ser encontrada em muitas referências. No caso de jatos de primeira ordem, denotado aqui por $J E$, ela é particularmente simples. Dado um fibrado $E$ sobre $M$ com projeção $\pi$ e fibra típica $Q$, a fibra de $J E$ em cada ponto $e \in E$ sobre o ponto $x \in M$ é definida por

$$
J_{e} E=\left\{u \in L\left(T_{x} M, T_{e} E\right): T_{e} \pi \circ u=\mathrm{id}_{T_{x} M}\right\} .
$$

Assim, os elementos de $J_{e} E$ são exatamente os candidatos para as aplicações tangentes em $x$ de seções (locais) $\varphi$ do fibrado $E$ satisfazendo $\varphi(x)=e$. Obviamente, $J_{e} E$ é um subespaço afim do espaço vetorial $L\left(T_{x} M, T_{e} E\right)$ de todas as aplicações lineares do espaço tangente $T_{x} M$ para o espaço tangente $T_{e} E$, sendo o correspondente espaço vetorial de diferenças o espaço vetorial $L\left(T_{x} M, V_{e} E\right)$ de todas as aplicações lineares do espaço tangente $T_{x} M$ para o espaço vertical $V_{e} E$ :

$$
\overrightarrow{J_{e}} E=L\left(T_{x} M, V_{e} E\right) \cong T_{x}^{*} M \otimes V_{e} E .
$$

Variando $e$ em $E$, obtemos um fibrado afim sobre $E$ em relação à projeção alvo $\tau: J E \rightarrow E$, que é um subfibrado do fibrado vetorial $L\left(\pi^{*} T M, T E\right) \cong \pi^{*}\left(T^{*} M\right) \otimes T E$, sendo o correspondente fibrado vetorial de diferenças o fibrado vetorial sobre $E$ em relação à projeção alvo $\vec{\tau}: \vec{J} E \rightarrow E$, dado por

$$
\vec{J} E=L\left(\pi^{*}(T M), V E\right) \cong \pi^{*}\left(T^{*} M\right) \otimes V E .
$$

Em geral, $J E$ e $\vec{J} E$ também são fibrados sobre $M$ em relação às respectivas projeções fonte $\sigma=\pi \circ \tau: J E \rightarrow M$ e $\vec{\sigma}=\pi \circ \vec{\tau}: \vec{J} E \rightarrow M$, porém apenas fibrados gerais sem estrutura adicional. Ocasionalmente, denotaremos $J E$ por $J_{E} E$ ou por $J_{M} E$ e $\vec{J} E$ por $\vec{J}_{E} E$ ou por $\vec{J}_{M} E$ para enfatizar que estamos considerando-o como fibrado em relação à projeção alvo ou em relação à projeção fonte, respectivamente. Também faremos uso do fato de que os pontos de $J E$ podem ser representados como 1 -jatos de seções (locais) de $E$ : para todo ponto $e \in E$ sobre o ponto $x \in M$, os elementos de $J_{e} E$ podem ser escritos na forma $u=j_{x} \varphi$ onde $\varphi$ é uma seção de $E$ definida em uma vizinhança aberta de $x$ em $M$ tal que $\varphi(x)=e$ e $T_{x} \varphi=u$.

$N a$ discussão a seguir, tornar-se-á importante que estruturas adicionais sobre o fibrado $E$ possam ser herdadas pelo fibrado $J_{M} E$. Por exemplo, se $E$ for um fibrado vetorial/afim sobre $M$ então $J E$ também será um fibrado vetorial/afim sobre $M$. Mais especificamente, é fácil ver que se $E$ for um fibrado vetorial sobre $M$, então $J_{M} E$ também o será: basta definir

$$
\lambda j_{x} \varphi+\mu j_{x} \psi=j_{x}(\lambda \varphi+\mu \psi) \quad \text { para } \lambda, \mu \in \mathbb{R}, \varphi, \psi \in \Gamma(E) .
$$

De modo semelhante, se $E$ for um fibrado afim sobre $M$ e $\vec{E}$ for o correspondente fibrado vetorial de diferenças, então $J_{M} E$ também será um fibrado afim sobre $M$ e $J_{M} \vec{E}$ será o 
correspondente fibrado vetorial de diferenças: basta definir

$$
j_{x} \varphi+j_{x} \vec{\varphi}=j_{x}(\varphi+\vec{\varphi}) \quad \text { para } \varphi \in \Gamma(E), \vec{\varphi} \in \Gamma(\vec{E}) .
$$

Durante o desenvolvimento deste trabalho usaremos sistematicamente cálculos em coordenadas locais. Tipicamente, denotaremos coordenadas locais na base $M$ por $x^{\mu}$, coordenadas locais na fibra típica $Q$ por $q^{i}$ e as coordenadas locais no espaço total $E$ induzidas por estas e uma escolha de trivialização local por $\left(x^{\mu} ; q^{i}\right) .{ }^{3}$ Estas por sua vez induzem coordenadas locais em $J E$ e em $\vec{J} E$, que escrevemos na forma $\left(x^{\mu}, q^{i} ; q_{\mu}^{i}\right)$ ou $\left(x^{\mu}, q^{i} ; \vec{q}_{\mu}^{i}\right)$ quando consideramos $J E$ ou $\overrightarrow{J E}$ como fibrado sobre $E$, na forma $\left(x^{\mu} ; q^{i}, q_{\mu}^{i}\right)$ ou $\left(x^{\mu} ; q^{i}, \vec{q}_{\mu}^{i}\right)$ quando consideramos $J E$ ou $\overrightarrow{J E}$ como fibrado sobre $M$ e na forma $\left(x^{\mu}, q^{i}, q_{\mu}^{i}\right)$ ou $\left(x^{\mu}, q^{i}, \vec{q}_{\mu}^{i}\right)$ quando não for necessário ou conveniente especificar a projeção. Combinando uma mudança de coordenadas locais $x^{\mu} \rightarrow x^{\prime \kappa}$ em $M$, uma mudança de coordenadas locais $q^{i} \rightarrow q^{\prime k}$ em $Q$ e uma mudança de trivialização local, obtemos uma mudança de coordenadas locais $\left(x^{\mu}, q^{i}\right) \rightarrow\left(x^{\prime \kappa}, q^{\prime k}\right)$ em $E$ onde

$$
x^{\prime \kappa}=x^{\prime \kappa}(x), \quad q^{\prime k}=q^{\prime k}(x, q) .
$$

Esta por sua vez induz mudanças de coordenadas locais $\left(x^{\mu}, q^{i}, q_{\mu}^{i}\right) \rightarrow\left(x^{\prime \kappa}, q^{\prime k}, q_{\kappa}^{\prime k}\right)$ em $J E$ e $\left(x^{\mu}, q^{i}, \vec{q}_{\mu}^{i}\right) \rightarrow\left(x^{\prime \kappa}, q^{\prime k}, \vec{q}_{\kappa}^{\prime k}\right)$ em $\vec{J} E$ que são dadas por

$$
q_{\kappa}^{\prime k}=\frac{\partial q^{\prime k}}{\partial q^{i}} \frac{\partial x^{\mu}}{\partial x^{\prime \kappa}} q_{\mu}^{i}+\frac{\partial q^{\prime k}}{\partial x^{\mu}} \frac{\partial x^{\mu}}{\partial x^{\prime \kappa}},
$$

$\mathrm{e}$

$$
\vec{q}_{\kappa}^{\prime k}=\frac{\partial q^{\prime k}}{\partial q^{i}} \frac{\partial x^{\mu}}{\partial x^{\prime k}} \vec{q}_{\mu}^{i}
$$

Em particular, se $E$ for um fibrado vetorial/afim sobre $M$ e os $q^{i}$ assim como os $q^{\prime k}$ forem coordenadas lineares/afins ao longo das fibras, então a mudança de coordenadas corresponde a uma transformação de calibre, efetuada por uma matriz $\left(g_{i}^{k}\right)$ de funções, conforme

$$
q^{\prime k}(x, q)=g_{i}^{k}(x) q^{i}
$$

no primeiro caso e por uma matriz $\left(g_{i}^{k}\right)$ mais um vetor $\left(g^{k}\right)$ de funções, conforme

$$
q^{\prime k}(x, q)=g_{i}^{k}(x) q^{i}+g^{k}(x)
$$

no segundo caso. Em ambos os casos,

$$
\frac{\partial q^{\prime k}}{\partial q^{i}}(x, q)=g_{i}^{k}(x)
$$

\footnotetext{
${ }^{3}$ Usamos o símbolo ";" para sinalizar a separação entre "coordenadas horizontais" e "coordenadas verticais", em relação à uma projeção dada.
} 
e portanto

$$
q_{\kappa}^{\prime k}=g_{i}^{k} \frac{\partial x^{\mu}}{\partial x^{\prime \kappa}} q_{\mu}^{i}+\partial_{\mu} g_{i}^{k} \frac{\partial x^{\mu}}{\partial x^{\prime \kappa}} q^{i}
$$

ou

$$
q_{\kappa}^{\prime k}=g_{i}^{k} \frac{\partial x^{\mu}}{\partial x^{\prime \kappa}} q_{\mu}^{i}+\partial_{\mu} g_{i}^{k} \frac{\partial x^{\mu}}{\partial x^{\prime \kappa}} q^{i}+\partial_{\mu} g^{k} \frac{\partial x^{\mu}}{\partial x^{\prime \kappa}}
$$

respectivamente, confirmando assim a afirmação acima de que $E$ sendo um fibrado vetorial/afim sobre $M$ então $J E$ também é.

\subsubsection{Levantamento canônico}

O próximo passo a ser discutido é o levantamento canônico de automorfismos e, passando para grupos a um parâmetro, o levantamento canônico de automorfismos infinitesimais, i.e., de campos vetoriais projetáveis, de $E$ para $\vec{J} E$ e para $J E$. No final discutiremos a ação destes levantamentos sobre seções de $\vec{J} E$ e de $J E$.

Um automorfismo do fibrado $E$ pode ser levantado para um automorfismo do fibrado dos jatos linearizados $\vec{J} E$, como fibrado vetorial sobre $E$, definindo $\Phi_{\vec{J} E}: \vec{J} E \rightarrow \vec{J} E$ como segue: dado um ponto $e \in E$ sobre o ponto $x \in M$ e uma aplicação linear $\vec{u}_{e} \in \vec{J}_{e} E=$ $L\left(T_{x} M, V_{e} E\right)$, definimos $\Phi_{\vec{J} E}\left(\vec{u}_{e}\right) \in \vec{J}_{\Phi_{E}(e)} E=L\left(T_{\Phi_{M}(x)} M, V_{\Phi_{E}(e)} E\right)$ por

$$
\Phi_{\overrightarrow{J E}}\left(\vec{u}_{e}\right)=T_{e} \Phi_{E} \circ \vec{u}_{e} \circ\left(T_{x} \Phi_{M}\right)^{-1} .
$$

Esta fórmula define uma aplicação linear de $L\left(T_{x} M, V_{e} E\right)$ para $L\left(T_{\Phi_{M}(x)} M, V_{\Phi_{E}(e)} E\right)$. Em particular, o seguinte diagrama comuta:

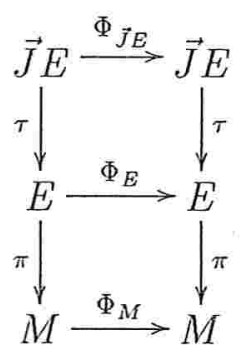

De forma análoga, um automorfismo do fibrado $E$ pode ser levantado para um automorfismo do fibrado dos jatos $J E$, como fibrado afim sobre $E$, definindo $\Phi_{J E}: J E \rightarrow J E$ como segue: dado um ponto $e \in E$ sobre o ponto $x \in M$ e um jato $u_{e} \in J_{e} E$, definimos $\Phi_{J E}\left(u_{e}\right) \in J_{\Phi_{E}(e)} E$ por

$$
\Phi_{J E}\left(u_{e}\right)=T_{e} \Phi_{E} \circ u_{e} \circ\left(T_{x} \Phi_{M}\right)^{-1}
$$


Esta fórmula define uma aplicação linear de $L\left(T_{x} M, T_{e} E\right)$ para $L\left(T_{\Phi_{M}(x)} M, T_{\Phi_{E}(e)} E\right)$ que, por restrição, induz uma aplicação afim de $J_{e} E$ para $J_{\Phi_{E}(e)} E$, já que mapeia $J_{e} E$ para $J_{\Phi_{E}(e)} E$ :

$$
\begin{aligned}
T_{\Phi_{E}(e)} \pi_{E} \circ \Phi_{J E}\left(u_{e}\right) & =T_{\Phi_{E}(e)} \pi_{E} \circ T_{e} \Phi_{E} \circ u_{e} \circ\left(T_{x} \Phi_{M}\right)^{-1} \\
& =T_{e}\left(\pi_{E} \circ \Phi_{E}\right) \circ u_{e} \circ\left(T_{x} \Phi_{M}\right)^{-1} \\
& =T_{e}\left(\Phi_{M} \circ \pi_{E}\right) \circ u_{e} \circ\left(T_{x} \Phi_{M}\right)^{-1} \\
& =T_{x} \Phi_{M} \circ T_{e} \pi_{E} \circ u_{e} \circ\left(T_{x} \Phi_{M}\right)^{-1} \\
& =T_{x} \Phi_{M} \circ \mathrm{id}_{T_{x} M} \circ\left(T_{x} \Phi_{M}\right)^{-1} \\
& =\mathrm{id}_{T_{\Phi_{M}(x)}} M
\end{aligned}
$$

Em particular, o seguinte diagrama comuta:

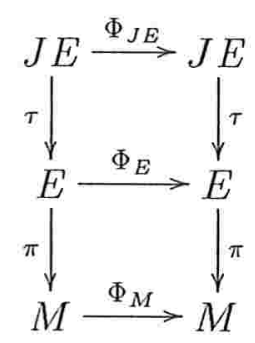

Passando à descrição da situação infinitesimal, podemos reduzir o levantamento de automorfismos infinitesimais ao levantamento de grupos (locais) a um parâmetro de automorfismos (locais). Assim, todo campo vetorial projetável $X_{E}$ sobre $E$ possui levantamentos canônicos para campos vetoriais projetáveis $X_{\vec{J} E}$ sobre $\vec{J} E$ e $X_{J E}$ sobre $J E$. Ao invés de fórmulas globais que são pouco instrutivas, apresentamos as expressões em coordenadas locais adaptadas, que podem ser deduzidas se observarmos que localmente, automorfismos são representadas por transformações de um sistema de coordenadas locais adaptadas para outro e portanto podemos calcular seus geradores por diferenciação em relação a um parâmetro de fluxo $\lambda$. Explicitamente, escrevendo

$\mathrm{e}$

$$
X_{M}=X^{\mu} \frac{\partial}{\partial x^{\mu}}
$$

$$
X_{E}=X^{\mu} \frac{\partial}{\partial x^{\mu}}+X^{i} \frac{\partial}{\partial q^{i}}
$$

onde os $X^{\mu}$ dependem somente das coordenadas $x^{\rho}$ na variedade base, e substituindo a equação (1.22) por

$$
x^{\prime \kappa}=x^{\prime \kappa}(x, \lambda) \quad, \quad q^{\prime k}=q^{\prime k}(x, q, \lambda),
$$

podemos calcular os componentes

$$
X^{\kappa}=\left.\frac{\partial x^{\prime \kappa}}{\partial \lambda}\right|_{\lambda=0}, \quad X^{k}=\left.\frac{\partial q^{\prime k}}{\partial \lambda}\right|_{\lambda=0}
$$


$\mathrm{e}$

$$
\vec{X}_{\kappa}^{k}=\left.\frac{\partial \vec{q}_{\kappa}^{\prime k}}{\partial \lambda}\right|_{\lambda=0}, \quad X_{\kappa}^{k}=\left.\frac{\partial q_{\kappa}^{\prime k}}{\partial \lambda}\right|_{\lambda=0}
$$

de $X_{\vec{J} E}$ e de $X_{J E}$ por diferenciação das equações (1.24) e (1.23), respectivamente, em relação a $\lambda$, usando que

$$
\left.\frac{\partial x^{\prime \kappa}}{\partial x^{\mu}}\right|_{\lambda=0}=\delta_{\mu}^{\kappa},\left.\frac{\partial q^{\prime k}}{\partial q^{i}}\right|_{\lambda=0}=\delta_{i}^{k},\left.\frac{\partial q^{\prime k}}{\partial x^{\mu}}\right|_{\lambda=0}=0
$$

$\mathrm{e}$

$$
\left.\frac{\partial}{\partial \lambda}\left(\frac{\partial x^{\mu}}{\partial x^{\prime \kappa}}\right)\right|_{\lambda=0}=-\left.\left.\left.\frac{\partial x^{\mu}}{\partial x^{\prime \rho}}\right|_{\lambda=0} \frac{\partial}{\partial \lambda}\left(\frac{\partial x^{\prime \rho}}{\partial x^{\sigma}}\right)\right|_{\lambda=0} \frac{\partial x^{\sigma}}{\partial x^{\prime \kappa}}\right|_{\lambda=0}=-\frac{\partial X^{\mu}}{\partial x^{\kappa}} .
$$

O resultado é que

$$
X_{\vec{J} E}=X^{\mu} \frac{\partial}{\partial x^{\mu}}+X^{i} \frac{\partial}{\partial q^{i}}+\left(\frac{\partial X^{i}}{\partial q^{j}} \vec{q}_{\mu}^{j}-\frac{\partial X^{\nu}}{\partial x^{\mu}} \vec{q}_{\nu}^{i}\right) \frac{\partial}{\partial \vec{q}_{\mu}^{i}},
$$

$\mathrm{e}$

$$
X_{J E}=X^{\mu} \frac{\partial}{\partial x^{\mu}}+X^{i} \frac{\partial}{\partial q^{i}}+\left(\frac{\partial X^{i}}{\partial q^{j}} q_{\mu}^{j}-\frac{\partial X^{\nu}}{\partial x^{\mu}} q_{\nu}^{i}+\frac{\partial X^{i}}{\partial x^{\mu}}\right) \frac{\partial}{\partial q_{\mu}^{i}} .
$$

Finalmente, a ação de automorfismos e automorfismos infinitesimais sobre seções de $\vec{J} E$ e de $J E$ é definida pela ação dos correspondentes levantamentos. Em particular, a ação de um automorfismo infinitesimal $X=\left(X_{J E}, X_{E}, X_{M}\right)$ sobre uma seção $j \varphi=(\varphi, \partial \varphi)$ de $J E$ fornece uma seção $\delta_{X}(j \varphi)=\left(\delta_{X} \varphi, \delta_{X} \partial \varphi\right)$ do fibrado vetorial $(j \varphi)^{*}(V(J E))$ sobre $M$ que é o pull-back do fibrado vertical $V(J E)$ de $J E$ via $j \varphi$ :

$$
\delta_{X}(j \varphi)(x)=X_{J E}(j \varphi(x))-T_{x}(j \varphi) \cdot X_{M}(x) \text { para } x \in M .
$$

Em coordenadas locais adaptadas, esta fórmula reproduz a equação (1.16) em conjunto com a equação

$$
\delta_{X} \partial_{\mu} \varphi^{i}=\frac{\partial X^{i}}{\partial q^{j}}(\varphi) \partial_{\mu} \varphi^{j}-\frac{\partial X^{\nu}}{\partial x^{\mu}}(\varphi) \partial_{\nu} \varphi^{i}+\frac{\partial X^{i}}{\partial x^{\mu}}(\varphi)-\partial_{\nu} \partial_{\mu} \varphi^{i} X^{\nu}
$$

\subsection{Fibrados de cojatos}

Assim como o fibrado tangente de uma variedade $M$, o fibrado dos jatos de primeira ordem de um fibrado $E$ admite uma noção natural de dual.

Primeiro, introduzimos o dual afim $J^{\star} E$ de $J E$, que é semelhante ao dual linear $\vec{J}^{*} E$ de $\overrightarrow{J E}$ : ambos são fibrados vetoriais sobre $E$ cujas fibras em cada ponto $e$ de $E$ são definidas por

$$
J_{e}^{\star} E=\left\{z_{e}: J_{e} E \longrightarrow \mathbb{R} \text { afim }\right\},
$$


$\mathrm{e}$

$$
\vec{J}_{e}^{*} E=\left\{\vec{z}_{e}: \vec{J}_{e} E \longrightarrow \mathbb{R} \text { linear }\right\},
$$

respectivamente. Entretanto, para chegar ao tipo de dual que aparece naturalmente na teoria dos campos, torna-se necessário substituir, no codomínio das aplicações afins ou lineares empregadas, a reta real pelo espaço unidimensional das formas de volume sobre o ponto correspondente de $M$, ou seja, considerar o dual afim torcido $J^{\circledR} E$ de $J E$ e o dual linear torcido $\vec{J}{ }^{\circledast} E$ de $\vec{J} E$ cujas fibras em cada ponto $e$ de $E$ são definidas por

$$
J_{e}^{\circledast} E=\left\{z_{e}: J_{e} E \longrightarrow \bigwedge^{n} T^{*} M \text { afim }\right\},
$$

e

$$
\vec{J}_{e}^{\circledast} E=\left\{\vec{z}_{e}: \vec{J}_{e} E \longrightarrow \bigwedge^{n} T^{*} M \text { linear }\right\},
$$

respectivamente. Em outras palavras, temos

$$
J^{\circledast} E=J^{\star} E \otimes \bigwedge^{n} T^{*} M,
$$

e

$$
\vec{J}^{\circledast} E=\vec{J}^{*} E \otimes \bigwedge^{n} T^{*} M,
$$

respectivamente. Como os fibrados $J E$ e $\vec{J} E$ introduzidos na seção anterior, cada um destes fibrados admite duas projeções: uma projeção alvo, em relação à qual é um fibrado vetorial, e uma projeção fonte, em relação à qual não admite, em geral, nenhuma estrutura adicional.

Em termos de coordenadas locais, podemos afirmar que as coordenadas locais $\left(x^{\mu}, q^{i}, q_{\mu}^{i}\right)$ de $J E$ e $\left(x^{\mu}, q^{i} ; \vec{q}_{\mu}^{i}\right)$ introduzidas na seção anterior induzem coordenadas locais $\left(x^{\mu}, q^{i}, p_{i}^{\mu}, p\right)$ para $J^{\star} E$ e $J^{\oplus} E$ e coordenadas locais $\left(x^{\mu}, q^{i}, p_{i}^{\mu}\right)$ para $\vec{J}^{*} E$ e $\vec{J}^{\circledast} E$, definidas pelo requerimento de que o pareamento entre um ponto de $J E$ e um ponto do seu dual afim seja dado por

$$
p_{i}^{\mu} q_{\mu}^{i}+p
$$

no caso não-torcido e por

$$
\left(p_{i}^{\mu} q_{\mu}^{i}+p\right) d^{n} x
$$

no caso torcido, enquanto que o pareamento entre um ponto de $\vec{J} E$ e um ponto do seu dual linear seja dado por

$$
p_{i}^{\mu} \vec{q}_{\mu}^{i}
$$

no caso não-torcido e por

$$
p_{i}^{\mu} \vec{q}_{\mu}^{i} d^{n} x
$$

no caso torcido. Assim, combinando uma mudança de coordenadas locais $x^{\mu} \rightarrow x^{\prime \kappa}$ em $M$, uma mudança de coordenadas locais $q^{i} \rightarrow q^{\prime k}$ em $Q$ e uma mudança de trivialização local, obtemos, além das mudanças de coordenadas locais induzidas já discutidas na seção anterior, 
mudanças de coordenadas locais induzidas $\left(x^{\mu}, q^{i}, p_{i}^{\mu}, p\right) \rightarrow\left(x^{\prime \kappa}, q^{\prime k}, p_{k}^{\prime \kappa}, p^{\prime}\right)$ em $J^{\star} E$ e em $J^{\circledast} E$ e $\left(x^{\mu}, q^{i}, p_{i}^{\mu}\right) \rightarrow\left(x^{\prime \kappa}, q^{\prime k}, p_{k}^{\prime \kappa}\right)$ em $\vec{J}^{*} E$ e em $\vec{J}^{\circledast} E$ que são dadas por

$$
p_{k}^{\prime \kappa}=\frac{\partial x^{\prime \kappa}}{\partial x^{\mu}} \frac{\partial q^{i}}{\partial q^{\prime k}} p_{i}^{\mu} \quad, \quad p^{\prime}=p-\frac{\partial q^{\prime k}}{\partial x^{\mu}} \frac{\partial q^{i}}{\partial q^{\prime k}} p_{i}^{\mu} .
$$

no caso não-torcido e

$$
p_{k}^{\prime \kappa}=\operatorname{det}\left(\frac{\partial x}{\partial x^{\prime}}\right) \frac{\partial x^{\prime \kappa}}{\partial x^{\mu}} \frac{\partial q^{i}}{\partial q^{\prime k}} p_{i}^{\mu} \quad, \quad p^{\prime}=\operatorname{det}\left(\frac{\partial x}{\partial x^{\prime}}\right)\left(p-\frac{\partial q^{\prime k}}{\partial x^{\mu}} \frac{\partial q^{i}}{\partial q^{\prime k}} p_{i}^{\mu}\right) .
$$

no caso torcido.

Uma observação importante na geometria multissimplética é que o fibrado $J^{\otimes} E$ é isomorfo a um certo subfibrado vetorial do fibrado $\bigwedge^{n} T^{*} E$ das $n$-formas sobre $E$, a saber o subfibrado vetorial $\bigwedge_{n-1}^{n} T^{*} E$ das $n$-formas $(n-1)$-horizontais sobre $E$. Explicitamente, este é definido por

$$
\bigwedge_{n-1}^{n} T_{e}^{*} E=\left\{z \in \bigwedge^{n} T_{e}^{*} E / i_{u} i_{v} z=0 \text { para todo } u, v \in V_{e} E\right\} .
$$

De fato, existe um isomorfismo canônico

$$
\Psi: \bigwedge_{n-1}^{n} T^{*} E \stackrel{\cong}{\longrightarrow} J^{\circledast} E
$$

de fibrados vetoriais sobre $E$ que pode ser definido explicitamente da seguinte maneira: dado um ponto $e \in E$ sobre o ponto $x \in M$ e uma $n$-forma $(n-1)$-horizontal $\alpha_{e} \in \bigwedge_{n-1}^{n} T_{e}^{*} E$ qualquer, juntamente com um jato $u_{e} \in J_{e} E$, podemos usar $u_{e}$, que é uma aplicação linear de $T_{x} M$ para $T_{e} E$, para fazer o pull-back da $n$-forma $\alpha_{e}$ sobre $T_{e} E$ para uma $n$-forma $u_{e}^{*} \alpha_{e}$ sobre $T_{x} M$. Obviamente, $u_{e}^{*} \alpha_{e}$ é uma função afim em relação a $u_{e}$ quando $u_{e}$ percorre o espaço afim $J_{e} E$ uma vez que na verdade ela é uma função linear de $u_{e}$ quando $u_{e}$ percorre todo o espaço vetorial $L\left(T_{x} M, T_{e} E\right.$ ) (a restrição do domínio de uma aplicação linear entre dois espaços vetoriais a um subespaço afim define uma aplicação afim). Desta forma, colocando

$$
\Psi_{e}\left(\alpha_{e}\right) \cdot u_{e}=u_{e}^{*} \alpha_{e}
$$

definimos uma aplicação $\Psi_{e}: \bigwedge_{n-1}^{n} T_{e}^{*} E \rightarrow J_{e}^{\star} E$ que é evidentemente linear e, como $e$ varia sobre $E$, providencia o isomorfismo (1.52) desejado. Notemos que para sistemas com apenas um grau de liberdade interna, i.e., quando $N=1$, a condição de $(n-1)$-horizontalidade é automática, já que neste caso, quaisquer dois vetores verticais são proporcionais:

$$
\bigwedge_{n-1}^{n} T^{*} E=\bigwedge^{n} T^{*} E \quad \text { quando } N=1
$$




\subsubsection{Levantamento canônico}

O próximo passo a ser discutido é o levantamento canônico de automorfismos e, passando para grupos a um parâmetro, o levantamento canônico de automorfismos infinitesimais, i.e., de campos vetoriais projetáveis, para o fibrado dual linear (torcido ou não) de $\vec{J} E$ e o fibrado dual afim (torcido ou não) de $J E$, que é definido simplesmente por dualização dos automorfismos obtidos na seção anterior. No final discutiremos a ação destes levantamentos sobre seções destes fibrados duais.

Primeiro, dualização linear do automorfismo $\Phi_{\vec{J} E}: \vec{J} E \rightarrow \overrightarrow{J E}$ de $\vec{J} E$ obtido por levantamento de um automorfismo $\Phi_{E}: E \rightarrow E$ de $E$ fornece automorfismos $\Phi_{\vec{J}^{*} E}: \vec{J}^{*} E \rightarrow \vec{J}^{*} E$ e $\Phi_{\vec{J}_{E} E}: \vec{J}^{\circledast} E \rightarrow \vec{J}^{\circledast} E$ dos fibrados vetoriais $\vec{J}^{*} E$ e $\vec{J}^{\circledast} E$ sobre $E$ definidos como segue: dado um ponto $e \in E$ sobre o ponto $x \in M$ e $\vec{z}_{e} \in \vec{J}_{e}^{*} E$ ou $\vec{z}_{e} \in \vec{J}_{e}^{\circledast} E$, definimos $\Phi_{\vec{J}^{*} E}\left(\vec{z}_{e}\right) \in \vec{J}_{\Phi_{E}(e)} E$ ou $\Phi_{\vec{J}^{\circledast} E}\left(\vec{z}_{e}\right) \in \vec{J}_{\Phi_{E}(e)}^{\circledast} E$ por

$$
\Phi_{\vec{J}^{*} E}\left(\vec{z}_{e}\right)=\vec{z}_{e} \circ\left(\left.\Phi_{\vec{J} E}\right|_{\vec{J}_{e} E}\right)^{-1}
$$

ou

$$
\Phi_{\vec{J}^{\oplus} E}\left(\vec{z}_{e}\right)=\bigwedge^{n}\left(T_{x} \Phi_{M}\right)^{-1 *} \circ \vec{z}_{e} \circ\left(\left.\Phi_{\vec{J}_{E}}\right|_{\vec{J}_{e} E}\right)^{-1}
$$

respectivamente. Em particular, os seguintes diagramas comutam:
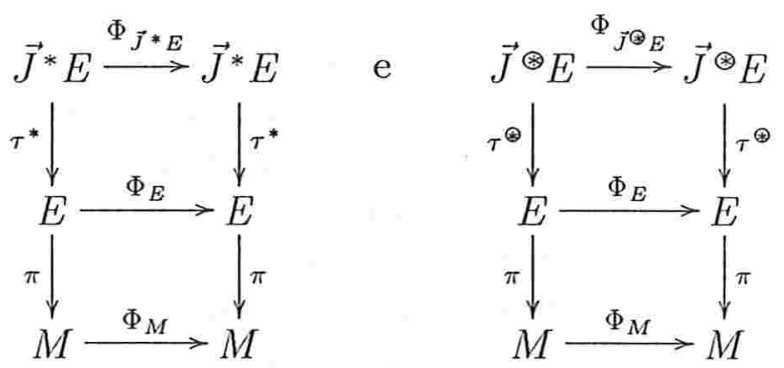

De forma análoga, dualização afim do automorfismo $\Phi_{J E}: J E \rightarrow J E$ de $J E$ obtido por levantamento de um automorfismo $\Phi_{E}: E \rightarrow E$ de $E$ fornece automorfismos $\Phi_{J^{\star} E}: J^{\star} E \rightarrow J^{\star} E$ e $\Phi_{J^{\oplus} E}: J^{\circledast} E \rightarrow J^{\circledast} E$ dos fibrados vetoriais $J^{\star} E$ e $J^{\circledast} E$ sobre $E$ definidos como segue: dado um ponto $e \in E$ sobre o ponto $x \in M$ e $z_{e} \in J_{e}^{\star} E$ ou $z_{e} \in J_{e}^{\oplus E}$, definimos $\Phi_{J^{\star} E}\left(z_{e}\right) \in J_{\Phi_{E}(e)}^{\star} E$ ou $\Phi_{J^{\oplus} E}\left(z_{e}\right) \in J_{\Phi_{E}(e)}^{\oplus} E$ por

$$
\Phi_{J^{\star} E}\left(z_{e}\right)=z_{e}^{\circ}\left(\left.\Phi_{J E}\right|_{J_{e} E}\right)^{-1}
$$

ou

$$
\Phi_{J^{\oplus} E}\left(z_{e}\right)=\bigwedge^{n}\left(T_{x} \Phi_{M}\right)^{-1 *} \circ z_{e} \circ\left(\left.\Phi_{J E}\right|_{J_{e} E}\right)^{-1}
$$


respectivamente. Em particular, os seguintes diagramas comutam:

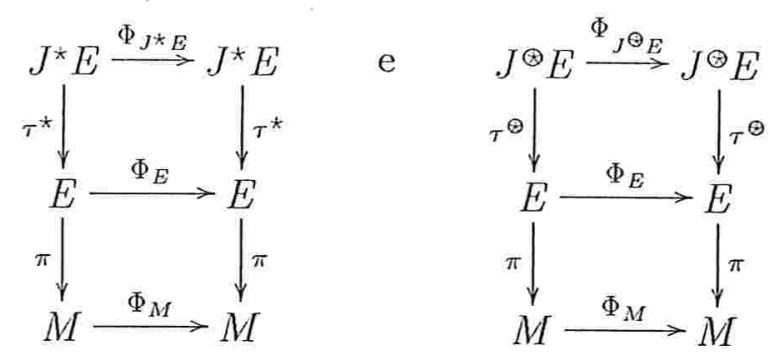

No último caso, deve ser notado que sob o isomorfismo canônico $\Psi$, o automorfismo $\Phi_{J^{\oplus} E}$ de $J^{\circledast} E$ corresponde ao automorfismo de $\bigwedge_{n-1}^{n} T^{*} E$ que age em $n$-formas sobre $E$ por pullback através de $\Phi_{E}^{-1}$. De fato, dado um ponto $e \in E$ sobre o ponto $x \in M$, uma $n$-forma $(n-1)$-horizontal $\omega_{e} \in \bigwedge_{n-1}^{n} T_{e}^{*} E$ sobre $T_{e} E$ e um jato $u_{\Phi_{E}(e)} \in J_{\Phi_{E}(e)} E$ em $\Phi_{E}(e)$, calculamos

$$
\begin{gathered}
\Phi_{\Phi^{\Theta} E}\left(\Psi_{e}\left(\omega_{e}\right)\right) \cdot u_{\Phi_{E}(e)}=\bigwedge^{n}\left(T_{x} \Phi_{M}\right)^{-1 *} \cdot\left(\Psi_{e}\left(\omega_{e}\right) \cdot \Phi_{J E}^{-1}\left(u_{\Phi_{E}}(e)\right)\right) \\
=\bigwedge^{n}\left(T_{x} \Phi_{M}\right)^{-1 *} \cdot\left(\left(\left(T_{e} \Phi_{E}\right)^{-1} \circ u_{\Phi_{E}}(e) \circ T_{x} \Phi_{M}\right)^{*} \omega_{e}\right) \\
=\left(\left(T_{e} \Phi_{E}\right)^{-1} \circ u_{\Phi_{E}(e)}\right)^{*} \omega_{e}=u_{\Phi_{E}(e)}^{*}\left(\left(T_{e} \Phi_{E}\right)^{-1 *} \omega_{e}\right) \\
=\Psi_{\Phi_{E}(e)}\left(\left(T_{e} \Phi_{E}\right)^{-1 *} \omega_{e}\right) \cdot u_{\Phi_{E}(e)} .
\end{gathered}
$$

Passando à descrição da situação infinitesimal, prosseguimos como antes, reduzindo o levantamento de automorfismos infinitesimais ao levantamento de grupos (locais) a um parâmetro de automorfismos (locais). Assim, todo campo vetorial projetável $X_{E}$ sobre $E$ possui levantamentos canônicos para campos vetoriais projetáveis $X_{\vec{J}^{*} E}$ sobre $\vec{J}^{*} E, X_{\vec{J}_{E} E}$ sobre $\vec{J}^{\circledast} E$, $X_{J^{\star} E}$ sobre $J^{\star} E$ e $X_{J^{\oplus} E}$ sobre $J^{\otimes} E$. Novamente, ao invés de fórmulas globais que são pouco instrutivas, apresentamos as expressões em coordenadas locais adaptadas, que podem ser deduzidas das leis de transformação (1.49) e (1.50) por diferenciação em relação a um parâmetro de fluxo $\lambda$, como antes. Usando as fórmulas anteriores, em conjunto com as relações

$$
\left.\frac{\partial}{\partial \lambda}\left(\frac{\partial q^{i}}{\partial q^{\prime k}}\right)\right|_{\lambda=0}=-\left.\left.\left.\frac{\partial q^{i}}{\partial q^{\prime r}}\right|_{\lambda=0} \frac{\partial}{\partial \lambda}\left(\frac{\partial q^{\prime r}}{\partial q^{s}}\right)\right|_{\lambda=0} \frac{\partial q^{s}}{\partial q^{\prime k}}\right|_{\lambda=0}=-\frac{\partial X^{i}}{\partial q^{k}}
$$

e

$$
\frac{\partial}{\partial \lambda} \operatorname{det}(A)=\operatorname{det}(A) \operatorname{tr}\left(A^{-1} \frac{\partial A}{\partial \lambda}\right)
$$

que implica

$$
\left.\frac{\partial}{\partial \lambda} \operatorname{det}\left(\frac{\partial x}{\partial x^{\prime}}\right)\right|_{\lambda=0}=-\frac{\partial X^{\mu}}{\partial x^{\mu}}
$$


obtemos

$$
X_{\vec{J}^{*} E}=X^{\mu} \frac{\partial}{\partial x^{\mu}}+X^{i} \frac{\partial}{\partial q^{i}}-\left(\frac{\partial X^{j}}{\partial q^{i}} p_{j}^{\mu}-\frac{\partial X^{\mu}}{\partial x^{\nu}} p_{i}^{\nu}\right) \frac{\partial}{\partial p_{i}^{\mu}}
$$

para $\vec{J}^{*} E$ e

$$
X_{\vec{J}^{\oplus E}}=X^{\mu} \frac{\partial}{\partial x^{\mu}}+X^{i} \frac{\partial}{\partial q^{i}}-\left(\frac{\partial X^{j}}{\partial q^{i}} p_{j}^{\mu}-\frac{\partial X^{\mu}}{\partial x^{\nu}} p_{i}^{\nu}+\frac{\partial X^{\nu}}{\partial x^{\nu}} p_{i}^{\mu}\right) \frac{\partial}{\partial p_{i}^{\mu}}
$$

para $\vec{J}{ }^{\circledast} E$, assim como

$$
X_{J^{\star} E}=X^{\mu} \frac{\partial}{\partial x^{\mu}}+X^{i} \frac{\partial}{\partial q^{i}}-\left(\frac{\partial X^{j}}{\partial q^{i}} p_{j}^{\mu}-\frac{\partial X^{\mu}}{\partial x^{\nu}} p_{i}^{\nu}\right) \frac{\partial}{\partial p_{i}^{\mu}}-\frac{\partial X^{i}}{\partial x^{\mu}} p_{i}^{\mu} \frac{\partial}{\partial p}
$$

para $J^{\star} E$ e

$$
\begin{aligned}
X_{J^{\oplus E}}=X^{\mu} \frac{\partial}{\partial x^{\mu}}+X^{i} \frac{\partial}{\partial q^{i}} & -\left(\frac{\partial X^{j}}{\partial q^{i}} p_{j}^{\mu}-\frac{\partial X^{\mu}}{\partial x^{\nu}} p_{i}^{\nu}+\frac{\partial X^{\nu}}{\partial x^{\nu}} p_{i}^{\mu}\right) \frac{\partial}{\partial p_{i}^{\mu}} \\
& -\left(\frac{\partial X^{i}}{\partial x^{\mu}} p_{i}^{\mu}+\frac{\partial X^{\nu}}{\partial x^{\nu}} p\right) \frac{\partial}{\partial p}
\end{aligned}
$$

para $J^{\circledast} E$. No último caso, nota-se que sob o isomorfismo canônico $\Psi$, o campo vetorial $X_{J^{\oplus} E}$ sobre $J^{\oplus} E$ corresponde ao campo vetorial sobre $\bigwedge_{n-1}^{n} T^{*} E$ que age em $n$-formas sobre $E$ pela derivada de Lie ao longo de $-X_{E}$. De fato, em termos de coordenadas locais adaptadas, temos que para todo campo vetorial $X_{E}$ sobre $E$ com representação (1.34) e toda $n$-forma $(n-1)$-horizontal $\omega_{E}$ sobre $E$ com representação

$$
\omega_{E}=\omega_{i}^{\mu} d q^{i} \wedge d^{n} x_{\mu}+\omega_{0} d^{n} x
$$

a derivada de Lie $L_{X_{E}} \omega_{E}$ de $\omega_{E}$ ao longo de $X_{E}$ é dada por

$$
\begin{aligned}
L_{X_{E}} \omega_{E}= & \left(X^{\kappa} \frac{\partial \omega_{i}^{\mu}}{\partial x^{\kappa}}+X^{k} \frac{\partial \omega_{i}^{\mu}}{\partial q^{k}}\right) d q^{i} \wedge d^{n} x_{\mu}+\left(X^{\kappa} \frac{\partial \omega_{0}}{\partial x^{\kappa}}+X^{k} \frac{\partial \omega_{0}}{\partial q^{k}}\right) d^{n} x \\
& +\left(\frac{\partial X^{j}}{\partial q^{i}} \omega_{j}^{\mu}-\frac{\partial X^{\mu}}{\partial x^{\nu}} \omega_{i}^{\nu}+\frac{\partial X^{\nu}}{\partial x^{\nu}} \omega_{i}^{\mu}+\frac{\partial X^{\mu}}{\partial q^{i}} \omega_{0}\right) d q^{i} \wedge d^{n} x_{\mu} \\
& +\left(\frac{\partial X^{i}}{\partial x^{\mu}} \omega_{i}^{\mu}+\frac{\partial X^{\nu}}{\partial x^{\nu}} \omega_{0}\right) d^{n} x \\
& +\frac{\partial X^{\mu}}{\partial q^{i}} \omega_{j}^{\nu} d q^{i} \wedge d q^{j} \wedge d^{n} x_{\mu \nu} .
\end{aligned}
$$

Essa fórmula como está é válida mesmo para campos vetoriais $X_{E}$ que não sejam projetáveis, mas ela também mostra que $X_{E}$ deve ser projetável se queremos garantir que tomando a 
derivada de Lie ao longo de $X_{E}$ leve $n$-formas $(n-1)$-horizontais em $n$-formas $(n-1)$ horizontais - exceto para sistemas com apenas um grau de liberdade interno: Quando $N=1$, a equação (1.54) assegura que campos vetoriais arbitrários sobre $E$ podem ser levantados para campos vetoriais sobre $J^{\circledR} E$, a única mudança sendo que, neste caso, a equação (1.64) possui um termo adicional, sendo substituída por

$$
\begin{aligned}
X_{J^{\oplus E} E}= & X^{\mu} \frac{\partial}{\partial x^{\mu}}+X^{i} \frac{\partial}{\partial q^{i}} \\
& -\left(\frac{\partial X^{j}}{\partial q^{i}} p_{j}^{\mu}-\frac{\partial X^{\mu}}{\partial x^{\nu}} p_{i}^{\nu}+\frac{\partial X^{\nu}}{\partial x^{\nu}} p_{i}^{\mu}+\frac{\partial X^{\mu}}{\partial q^{i}} p\right) \frac{\partial}{\partial p_{i}^{\mu}} \\
& -\left(\frac{\partial X^{i}}{\partial x^{\mu}} p_{i}^{\mu}+\frac{\partial X^{\nu}}{\partial x^{\nu}} p\right) \frac{\partial}{\partial p}
\end{aligned}
$$

onde os índices internos $i$ e $j$ tomam somente um único valor mas não obstante não foram suprimidos a fim de evitar uma confusão notacional.

Finalmente, a ação de automorfismos e automorfismos infinitesimais sobre seções é, mais uma vez, definida pela ação dos correspondentes levantamentos. Em particular, a ação de um automorfismo infinitesimal $X=\left(X_{\vec{J}^{\oplus} E}, X_{E}, X_{M}\right)$ sobre uma seção $\phi=(\varphi, \pi)$ de $\vec{J}^{\circledast} E$ fornece uma seção $\delta_{X} \phi=\left(\delta_{X} \varphi, \delta_{X} \pi\right)$ do fibrado vetorial $\phi^{*}\left(V\left(\vec{J}^{\circledR} E\right)\right)$ sobre $M$ que é o pull-back do fibrado vertical $V\left(\vec{J}^{\oplus} E\right)$ de $\vec{J}^{\oplus} E$ via $\phi$ :

$$
\delta_{X} \phi(x)=X_{\vec{J}^{\oplus} E}(\phi(x))-T_{x} \phi \cdot X_{M}(x) \quad \text { para } x \in M .
$$

Em coordenadas locais adaptadas, esta fórmula reproduz a equação (1.16) em conjunto com a equação

$$
\delta_{X} \pi_{i}^{\mu}=-\frac{\partial X^{j}}{\partial q^{i}}(\varphi) \pi_{j}^{\mu}+\frac{\partial X^{\mu}}{\partial x^{\nu}}(\varphi) \pi_{i}^{\nu}-\frac{\partial X^{\nu}}{\partial x^{\nu}}(\varphi) \pi_{i}^{\mu}-\partial_{\nu} \pi_{i}^{\mu} X^{\nu}
$$

De forma semelhante, se escolhermos uma seção

$$
\mathcal{H}: \vec{J}^{\circledast} E \rightarrow J^{\circledast} E
$$

de $J^{\circledast} E$ como fibrado afim em linhas sobre $\vec{J}^{\circledast} E$ (para a interpretação física deste tipo de seção como hamiltoniana, veja a seção seguinte), a ação de um automorfismo infinitesimal $X=\left(X_{J^{\oplus E}}, X_{E}, X_{M}\right)$ sobre uma seção $\tilde{\phi}=\mathcal{H}(\phi)=(\varphi, \pi,-H(\varphi, \pi))$ de $J^{\otimes} E$ fornece uma seção $\delta_{X} \tilde{\phi}=\left(\delta_{X} \varphi, \delta_{X} \pi,-\delta_{X} H(\varphi, \pi)\right)$ do fibrado vetorial $\tilde{\phi}^{*}\left(V\left(J^{\oplus} E\right)\right)$ sobre $M$ que é o pull-back do fibrado vertical $V\left(J^{\circledast E}\right)$ de $J^{\circledast} E$ via $\tilde{\phi}$ :

$$
\delta_{X} \tilde{\phi}(x)=X_{J^{\ominus} E}(\tilde{\phi}(x))-T_{x} \tilde{\phi} \cdot X_{M}(x) \quad \text { para } x \in M
$$


Em coordenadas locais adaptadas, esta fórmula reproduz as equações (1.16) e (1.69) em conjunto com a equação

$$
\delta_{X} H(\varphi, \pi)=\frac{\partial X^{i}}{\partial x^{\mu}}(\varphi) \pi_{i}^{\mu}-\frac{\partial}{\partial x^{\mu}}\left(X^{\mu} H(\varphi, \pi)\right)
$$

ou

$$
\begin{aligned}
\delta_{X} H(\varphi, \pi)= & \frac{\partial X^{i}}{\partial x^{\mu}}(\varphi) \pi_{i}^{\mu}-\frac{\partial X^{\mu}}{\partial x^{\mu}} H(\varphi, \pi) \\
& -X^{\mu} \frac{\partial H}{\partial x^{\mu}}(\varphi, \pi)-X^{\mu} \frac{\partial H}{\partial q^{i}}(\varphi, \pi) \partial_{\mu} \varphi^{i}-X^{\mu} \frac{\partial H}{\partial p_{j}^{\nu}}(\varphi, \pi) \partial_{\mu} \pi_{j}^{\nu} .
\end{aligned}
$$

\subsection{Geometria multissimplética}

Durante os últimos anos, evidenciou-se cada vez mais que o conceito matemático adequado para a formulação geométrica da teoria clássica dos campos é o de uma variedade multissimplética. No entanto, um dos primeiros problemas que enfrentamos neste contexto é a inexistência de uma definição consolidada deste conceito.

Uma definição preliminar seria a seguinte: Uma variedade multissimplética é uma variedade $P$ munida de uma forma diferencial $\omega$ fechada de grau $n+1$, onde $n$ é a dimensão do espaço-tempo $M$, e satisfazendo certas condições de natureza algébrica, entre elas no mínimo a de não-degenerescência: em cada ponto $p$ de $P$, a contração de $\omega_{p}$ com um vetor tangente $X_{p}$ não nulo fornece uma $n$-forma $i\left(X_{p}\right) \omega_{p}$ não nula. (Para $n=1$, recuperamos a definição padrão de uma variedade simplética da mecânica.) No entanto, esta condição de não-degenerescência não é suficiente para garantir a validade da versão multissimplética do teorema de Darboux [29]: este requer condições mais restritivas cuja natureza algébrica ainda não foi completamente esclarecida. Outro aspecto que não parece ser adequadamente contemplado na literatura é o fato de que as variedades multissimpléticas que ocorrem na teoria dos campos não são apenas variedades mas possuem a estrutura adicional de um fibrado sobre o espaço-tempo e que esta deve satisfazer uma condição de compatibilidade com a forma multissimplética. Destarte, a seguinte definição deve ser considerada um tanto provisória.

Definição 1.1 Uma variedade multissimplética é uma dupla $(P, \omega)$ onde $P$ é uma variedade e $\omega$ é uma forma diferencial de grau $n+1$ sobre $P$, chamada a forma multissimplética, que é fechada,

$$
d \omega=0
$$


e satifaz certas condições algébricas, entre elas no mínimo a de não-degenerescência, afirmando que para todo campo vetorial $X$, vale

$$
i_{X} \omega=0 \Longrightarrow X=0 \text {. }
$$

Definição 1.2 Uma variedade exatamente multissimplética é uma variedade $P$ munida de uma forma $\theta$ de grau $n$, chamada a forma multicanônica, tal que

$$
\omega=-d \theta
$$

é uma forma multissimplética sobre $P$.

Observa-se que, obviamente, a conclusão (1.74) deixa de ser válida para campos multivetoriais, ou seja, o núcleo de $\omega$ sobre multivetores não é trivial. (Isso ocorre mesmo para formas simpléticas, que se anulam sobre multivetores obtidos como produto exterior de vetores contidos em algum subespaço isotrópico. Da mesma forma, o núcleo de $\theta$ sobre multivetores não é trivial.) Dualmente, um papel especial e importante é desempenhado pelo aniquilador deste núcleo, ou seja, por formas cujo núcleo multivetorial é maior do que o de $\omega$, i.e., que se anulam sobre todos os multivetores contidos no núcleo de $\omega$. Em particular, em [12], foi adotada uma definição mais restritiva de variedade exatamente multissimplética, exigindo-se que o núcleo de $\omega$ seja contido no núcleo de $\theta$ :

$$
\operatorname{ker} \omega \subset \operatorname{ker} \theta \text {, }
$$

i.e., para todo campo multivetorial $\xi$, vale

$$
i_{\xi} \omega=0 \Longrightarrow i_{\xi} \theta=0 .
$$

A função principal desta restrição é garantir que o colchete de Poisson entre formas de Poisson definido em [12] seja bem definido. No entanto, para formas de grau $n-1$, a restrição a formas de Poisson torna-se desnecessária (e por este motivo nem aparece em [11]). Como no presente trabalho, consideramos apenas formas de grau $n-1$, optamos por adotar uma definição menos restritiva, o que permite incluir não apenas o espaço de multifase estendido mas também o espaço de multifase comum: ambos são exemplos de variedades exatamente multissimpléticas, desempenhando o mesmo papel na geometria multissimplética que os fibrados cotangentes na geometria simplética.

\subsubsection{Espaços de multifase}

Definição 1.3 Dado um fibrado de configuração E sobre o espaço-tempo $M$, o dual afim torcido $J^{\circledast E}$ do fibrado de jatos $J E$ de E é chamado o espaço de multifase estendido (associado a E) e o dual linear torcido $\vec{J}^{\circledast} E$ do fibrado de jatos linearizado $\vec{J} E$ de $E$ é chamada o espaço de multifase comum (associado a E). 
O espaço de multifase estendido $J{ }^{\circledast} E$ é o exemplo padrão de uma variedade exatamente multissimplética. A construção da forma multicanônica $\theta$ e da forma multissimplética $\omega$ sobre $J^{\circledast} E$ pode ser entendido da seguinte forma.

Seja $E$ uma variedade de dimensão $n+N$ e $\bigwedge^{n} T^{*} E$ o fibrado das $n$-formas sobre $E$, com projeção $\tau_{E}^{* n}$. Então existe uma $n$-forma canônica $\theta$ sobre $\bigwedge^{n} T^{*} E$, definida por

$$
\theta(z)\left(v_{1}, \ldots, v_{n}\right)=z\left(T_{z} \tau_{E}^{* n} \cdot v_{1}, \ldots, T_{z} \tau_{E}^{* n} \cdot v_{n}\right)
$$

onde $z \in \bigwedge^{n} T^{*} E$ e $v_{1}, \ldots, v_{n} \in T_{z}\left(\bigwedge^{n} T^{*} E\right)$. Em termos de coordenadas locais $y^{a}(1 \leqslant a \leqslant N)$ para $E$ e as coordenadas locais induzidas $\left(y^{a}, z_{a_{1} \ldots a_{n}}\right)\left(1 \leqslant a \leqslant N, 1 \leqslant a_{1}<\ldots<a_{n} \leqslant N\right)$ para $\bigwedge^{n} T^{*} E$, temos

$$
\theta=\frac{1}{n !} z_{a_{1} \ldots a_{n}} d y^{a_{1}} \wedge \ldots \wedge d y^{a_{n}}
$$

e portanto

$$
\omega=-\frac{1}{n !} d z_{a_{1} \ldots a_{n}} \wedge d y^{a_{1}} \wedge \ldots \wedge d y^{a_{n}} .
$$

Quando $E$ for o espaço total de um fibrado sobre $M$, podemos considerar a subvariedade $\bigwedge_{n-1}^{n} T^{*} E$ das $n$-formas $(n-1)$-horizontais sobre $E$ e restringir as formas $\theta$ e $\omega$ a esta subvariedade. Assim, usando o isomorfismo canônico 1.53, podemos transferir estas formas de $\bigwedge_{n-1}^{n} T^{*} E$ para $J^{\oplus E}$ : usaremos as mesmas letras $\theta$ e $\omega$, respectivamente, para todas estas formas. Em coordenadas locais adaptadas,

$$
\theta=p_{i}^{\mu} d q^{i} \wedge d^{n} x_{\mu}+p d^{n} x
$$

e portanto

$$
\omega=d q^{i} \wedge d p_{i}^{\mu} \wedge d^{n} x_{\mu}-d p \wedge d^{n} x
$$

Passando ao espaço de multifase comum $\vec{J}^{\circledast} E$, observamos que neste, não há a priori nenhuma forma multicanônica ou multissimplética preferida. No entanto, esta estrutura pode ser definida assim que escolhermos uma hamiltoniana. Ocorre que, como já foi devidamente enfatizado em [3], o conceito de hamiltoniana na geometria multissimplética exige o emprego de ambos os tipos de espaço de multifase, o estendido $J^{\circledast} E$ assim como o comum $\vec{J}^{\circledast} E$, sendo o primeiro um fibrado afim em linhas sobre o segundo em relação à projeção $\eta: J^{\circledast} E \rightarrow \vec{J}{ }^{\circledast} E$ que associa a cada forma afim sua parte linear.

Definição 1.4 Dado um fibrado de configuração E sobre o espaço-tempo $M$, uma hamiltoniana é uma seção

$$
\mathcal{H}: \vec{J}^{\circledast} E \longrightarrow J^{\circledast} E
$$

do espaço de multifase estendido $J{ }^{\oplus}$ E como fibrado afim em linhas sobre o espaço de multifase comum $\vec{J}{ }^{\circledast}$. 
Observemos que hamiltonianas sempre existem, uma vez que fibrados cuja fibra típica é difeomórfica a um $\mathbb{R}^{k}$ sempre admitem seções globais [22, Ch. 1, Theorem 5.7].

Dada uma hamiltoniana $\mathcal{H}$, podemos então definir uma $n$-forma $\theta_{\mathcal{H}}$ e uma $(n+1)$-forma $\omega_{\mathcal{H}}$ sobre $\vec{J} \otimes E$ por pull-back:

$$
\theta_{\mathcal{H}}=\mathcal{H}^{*} \theta, \omega_{\mathcal{H}}=\mathcal{H}^{*} \omega
$$

Em coordenadas locais adaptadas,

$$
\theta_{\mathcal{H}}=p_{i}^{\mu} d q^{i} \wedge d^{n} x_{\mu}-H d^{n} x
$$

e

$$
\omega_{\mathcal{H}}=d q^{i} \wedge d p_{i}^{\mu} \wedge d^{n} x_{\mu}+d H \wedge d^{n} x
$$

ou mais explicitamente,

$$
\omega_{\mathcal{H}}=d q^{i} \wedge d p_{i}^{\mu} \wedge d^{n} x_{\mu}+\frac{\partial H}{\partial q^{i}} d q^{i} \wedge d^{n} x+\frac{\partial H}{\partial p_{i}^{\mu}} d p_{i}^{\mu} \wedge d^{n} x .
$$

A observação crucial é que na teoria dos campos, ao contrário do que acontece na mecânica, a forma $\omega_{\mathcal{H}}$ é não-degenerada. De fato, usando coordenadas locais adaptadas, escrevemos um campo vetorial $\vec{X}$ qualquer sobre $\vec{J}^{\otimes} E$ na forma

$$
\vec{X}=X^{\mu} \frac{\partial}{\partial x^{\mu}}+X^{i} \frac{\partial}{\partial q^{i}}+X_{i}^{\mu} \frac{\partial}{\partial p_{i}^{\mu}}
$$

onde os coeficientes $X^{\mu}, X^{i}$ e $X_{i}^{\mu}$ são funções (em geral arbitrárias) das variáveis $x^{\kappa}, q^{k}$ e $p_{k}^{\kappa}$, e empregamos a forma explícita $(1.87)$ de $\omega_{\mathcal{H}}$ para obter

$$
\begin{aligned}
i_{\vec{X}} \omega_{\mathcal{H}}= & X^{\nu} d q^{i} \wedge d p_{i}^{\mu} \wedge d^{n} x_{\mu \nu}-\left(X_{i}^{\mu}+X^{\mu} \frac{\partial H}{\partial q^{i}}\right) d q^{i} \wedge d^{n} x_{\mu} \\
& +\left(X^{i} \delta_{\mu}^{\nu}-X^{\nu} \frac{\partial H}{\partial p_{i}^{\mu}}\right) d p_{i}^{\mu} \wedge d^{n} x_{\nu}+\left(X^{i} \frac{\partial H}{\partial q^{i}}+X_{i}^{\mu} \frac{\partial H}{\partial p_{i}^{\mu}}\right) d^{n} x .
\end{aligned}
$$

Quando $n>1$, o primeiro termo (que no caso $n=1$ está ausente) é linearmente independente dos demais e se anula apenas quando os coeficientes $X^{\mu}$ são zero, levando à conclusão de que a expressão inteira se anula se e somente se todos os coeficientes $X^{\mu}, X^{i}$ e $X_{i}^{\mu}$ são zero.

Uma diferença entre as formas multicanônicas e multissimpléticas sobre $J^{\circledR} E$ e sobre $\vec{J}{ }^{\circledast} E$ é que as primeiras satisfazem a relação (1.76) ou (1.77) entre os respectivos núcleos enquanto que as segundas não. Mas a principal diferença entre as duas estruturas é que a primeira é de natureza cinemática, independente da escolha de uma hamiltoniana, enquanto que a segunda é de natureza dinâmica, dependendo explicitamente da hamiltoniana. Este 
aspecto já pode ser notado na mecânica onde, na notação padrão empregada na primeira seção deste capítulo, as equações (1.81) e (1.82) se reduzem, respectivamente, às equações (1.5) e (1.6), enquanto que as equações (1.87) e (1.88) adquirem a forma

$$
\omega_{\mathcal{H}}=d q^{i} \wedge d p_{i}+\frac{\partial H}{\partial q^{i}} d q^{i} \wedge d t+\frac{\partial H}{\partial p_{i}} d p_{i} \wedge d t
$$

$\mathrm{e}$

$$
\vec{X}=X^{0} \frac{\partial}{\partial t}+X^{i} \frac{\partial}{\partial q^{i}}+X_{i} \frac{\partial}{\partial p_{i}} .
$$

Assim, a equação (1.89) é substituída por

$$
\begin{aligned}
i_{\vec{X}} \omega_{\mathcal{H}}= & -\left(X_{i}+X^{0} \frac{\partial H}{\partial q^{i}}\right) d q^{i}+\left(X^{i}-X^{0} \frac{\partial H}{\partial p_{i}}\right) d p_{i} \\
& +\left(X^{i} \frac{\partial H}{\partial q^{i}}+X_{i} \frac{\partial H}{\partial p_{i}}\right) d t .
\end{aligned}
$$

Neste caso vemos que a forma $\omega_{\mathcal{H}}$ possui um núcleo não-trivial, gerado pelo campo vetorial

$$
\frac{\partial}{\partial t}+\frac{\partial H}{\partial p_{i}} \frac{\partial}{\partial q^{i}}-\frac{\partial H}{\partial q^{i}} \frac{\partial}{\partial p_{i}}
$$

sendo que as equações de movimento podem ser expressas como o fato de que este campo vetorial deve se anular sobre as suas soluções.

Outro objeto importante que pode ser definido globalmente nos espaços de multifase (o comum assim como o estendido) é o campo de escalonamento ou campo de Euler que denotaremos por $\Sigma$. Esta definição é baseada exclusivamente no fato de que $\vec{J}^{\circledast} E$ e $J{ }^{\circledR} E$ são espaços totais de fibrados vetoriais sobre $E$. De fato, dado qualquer fibrado vetorial $V$ sobre $E$, $\Sigma_{V}$ (que iremos simplesmente denotar por $\Sigma$ quando não houver perigo de confusão) é definido como sendo o campo fundamental associado à ação de $\mathbb{R}$, considerado como um grupo comutativo pela adição, por transformações de escala, ou homotetias, nas fibras:

$$
\begin{aligned}
\mathbb{R} \times V & \longrightarrow V \\
(\lambda, v) & \longmapsto \exp (\lambda) v
\end{aligned}
$$

Assim $\Sigma$ é simplesmente o campo vetorial vertical em $V$ que, através da identificação do espaço tangente vertical a $V$ com a fibra típica do próprio $V$, torna-se a identidade em $V$ :

$$
\Sigma(v)=\left.\frac{d}{d \lambda} \exp (\lambda) v\right|_{\lambda=0}=v .
$$


Em coordenadas locais adaptadas, $\vec{\Sigma}$ sobre $\vec{J}^{\otimes} E$ é dado por

$$
\vec{\Sigma}=p_{i}^{\mu} \frac{\partial}{\partial p_{i}^{\mu}}
$$

enquanto que $\Sigma$ sobre $J^{\circledR} E$ é dado por

$$
\Sigma=p_{i}^{\mu} \frac{\partial}{\partial p_{i}^{\mu}}+p \frac{\partial}{\partial p}
$$

O segundo desempenha um papel particularmente importante na teoria, devido à seguinte

Proposição 1.1 A forma multicanônica $\theta$, a forma multissimplética $\omega$ e o campo de Euler $\Sigma$ no espaço de multifase estendido $J^{\oplus} E$ satisfazem as seguintes relações:

$$
\begin{aligned}
L_{\Sigma} \theta & =\theta . \\
L_{\Sigma} \omega & =\omega . \\
i_{\Sigma} \theta & =0 . \\
i_{\Sigma} \omega & =-\theta .
\end{aligned}
$$

DEmionstraÇÃo. Observemos que em relação à projeção alvo de $J^{\oplus} E$ para $E, \Sigma$ é vertical e $\theta$ é horizontal, o que implica a eq. (1.98). Em coordenadas locais adaptadas, observamos que a contração usual da forma multissimplética $\omega$, dada pela eq. (1.82), com o campo de Euler $\Sigma$, dado pela eq. (1.95), produz, a menos de sinal, a forma $\theta$, dada pela eq. (1.81), o que prova a eq. (1.99). Combinando estas duas equações, temos

$$
L_{\Sigma} \theta=d\left(i_{\Sigma} \theta\right)+i_{\Sigma} d \theta=-i_{\Sigma} \omega=\theta,
$$

provando a eq. (1.96). Finalmente,

$$
L_{\Sigma} \omega=d\left(i_{\Sigma} \omega\right)+i_{\Sigma} d \omega=-d \theta=\omega,
$$

provando a eq. (1.97).

Observe que a equação (1.99) concretiza a relação (1.76) ou (1.77) entre os núcleos de $\theta$ e de $\omega$ no espaço de multifase estendido, sendo que não existe nenhuma relação análoga entre os núcleos de $\theta_{\mathcal{H}}$ e de $\omega_{\mathcal{H}}$ no espaço de multifase comum (exceto quando $H$ depender linearmente dos multimomenta, o que é excluído pela hipótese de que o sistema seja nãodegenerado e mesmo quando este for degenerado constitui um caso pouco realista). 


\subsubsection{Levantamento hamiltoniano}

A projeção entre os espaços de multifase introduzida acima, assim como a correspondente projeção entre os duais não-torcidos, possui algumas propriedades adicionais que não são imediatamente visíveis a partir da afirmação de que em coordenadas locais adaptadas, elas são dadas pela simples eliminação $\left(x^{\mu}, q^{i}, p_{i}^{\mu}, p\right) \mapsto\left(x^{\mu}, q^{i}, p_{i}^{\mu}\right)$ da variável energia $p$, e que merecem uma análise mais cuidadosa. ${ }^{4}$ Estas propriedades adicionais baseiam-se na existência de uma ação natural

$$
\begin{aligned}
\mathbb{R} \times J^{\star} E & \longrightarrow J^{\star} E \\
(\alpha, z) & \longmapsto z+\alpha
\end{aligned}
$$

de $\mathbb{R}$ sobre $J^{\star} E$, definida pela identificação de formas constantes, como formas afins especiais, com seus valores. É fácil verificar que esta ação é livre e transitiva sobre as fibras de $J^{\star} E$ sobre $\overrightarrow{J^{*}} E$; portanto, $J^{\star} E$ é de fato um fibrado principal sobre $\vec{J}^{*} E$ em relação à projeção $\eta: J^{\star} E \rightarrow \vec{J}^{*} E$, com grupo estrutural $\mathbb{R}$. Em coordenadas locais adaptadas, o campo fundamental associado a esta ação é simplesmente o campo vetorial

$$
\frac{\partial}{\partial p}
$$

conferindo-lhe assim um significado global, independente de qualquer escolha de coordenadas. Também podemos transferir toda esta estrutura para os duais torcidos se escolhermos uma forma de volume $\epsilon$ no espaço-tempo $M$, pois esta estabelece isomorfismos $J^{\oplus} E \cong J^{\star} E$ e $\overrightarrow{J^{\circledast}} E \cong \vec{J}^{*} E$. Assim, obtemos uma ação

$$
\begin{aligned}
\mathbb{R} \times J^{\oplus} E & \longrightarrow J^{\circledast} E \\
(\alpha, z) & \longmapsto z+\alpha \epsilon
\end{aligned}
$$

de $\mathbb{R}$ sobre $J^{\circledast} E$ que torna $J^{\circledast} E$ um fibrado principal sobre $\vec{J}^{\circledast} E$ em relação à projeção $\eta: J^{\circledast} E \rightarrow \vec{J}^{\circledast} E$, com grupo estrutural $\mathbb{R}$. Em coordenadas locais adaptadas, o campo fundamental associado a esta ação é o campo vetorial

$$
\Sigma_{\epsilon}=\epsilon_{0} \frac{\partial}{\partial p} \quad \text { onde } \quad \epsilon=\epsilon_{0} d^{n} x
$$

Em particular, a mera existência de uma hamiltoniana implica que $J^{\circledast} E$, como fibrado principal sobre $\vec{J}^{\circledast} E$, é topologicamente trivial.

Esta estrutura torna-se interessante e útil quando consideramos o problema de levantar automorfismos e seus geradores de $\vec{J}^{\circledast} E$ para $J^{\circledast} E$ usando uma hamiltoniana $\mathcal{H}$ fixa.

\footnotetext{
${ }^{4}$ Para simplificar a notação, denotaremos essas duas projeções pelo mesmo símbolo $\eta$.
} 
Começamos pela questão do levantamento de automorfismos no caso dos duais nãotorcidos, supondo momentaneamente que seja dada uma seção $\mathcal{H}_{0}$ de $J^{\star} E$ sobre $\overrightarrow{J^{*}} E$. Neste caso, podemos afirmar que dado um automorfismo $\Phi_{\vec{J}^{*} E}: \vec{J}^{*} E \rightarrow \vec{J}^{*} E$ de $\vec{J}^{*} E$ sobre o difeomorfismo $\Phi_{M}: M \rightarrow M$ de $M$, existe um único automorfismo $\Phi_{J^{\star} E}: J^{\star} E \rightarrow J^{\star} E$ de $J^{*} E$ sobre o mesmo difeomorfismo $\Phi_{M}: M \rightarrow M$ de $M$ com as seguintes propriedades:

- $\Phi_{J^{\star} E}$ projeta sobre $\Phi_{\vec{J}^{*} E}$ sob a projeção $\eta$, i.e.,

$$
\eta \circ \Phi_{J^{\star} E}=\Phi_{\vec{J}^{*} E} \circ \eta
$$

- $\Phi_{J * E}$ é equivariante em relação à ação (1.100) de $\mathbb{R}$ :

$$
\begin{gathered}
\Phi_{J^{\star} E}(z+\alpha)=\Phi_{J^{\star} E}(z)+\alpha \\
\text { para } z \in J^{\star} E, \alpha \in \mathbb{R} .
\end{gathered}
$$

- $\Phi_{J^{*} E}$ é um levantamento de $\Phi_{\vec{J}^{*} E}$ pela seção $\mathcal{H}_{0}$, i.e.,

$$
\Phi_{J^{*} E} \circ \mathcal{H}_{0}=\mathcal{H}_{0} \circ \Phi_{\vec{J}^{*} E}
$$

Para demonstrar esta afirmação, notamos inicialmente que as primeiras duas propriedades significam que $\Phi_{J \star E}$ é um automorfismo de $J^{\star} E$ como fibrado principal sobre $\vec{J}^{*} E$. É bem conhecido e fácil provar que dado um fibrado principal trivial $P$ sobre uma variedade base $M$ com grupo estrutural $G$ e mediante a escolha de uma seção global $\sigma: M \rightarrow P$, um automorfismo $F: P \rightarrow P$ de $P$ que induz um determinado difeomorfismo $f: M \rightarrow M$ de $M$ pode ser representado por uma função $f_{0} \in C^{\infty}(M, G)$ determinada por $F(\sigma(m) \cdot g)=\sigma(f(m)) \cdot f_{0}(m) g$. Neste contexto, a escolha mais natural é dada por $f_{0} \equiv 1$, e é exatamente este levantamento cuja existência e unicidade está sendo afirmada, no caso especial em que $P, M, G$ e $\sigma$ são substituídos por $J^{\star} E, \vec{J}^{*} E, \mathbb{R}$ e $\mathcal{H}_{0}$, respectivamente.

Passando aos duais torcidos, definidos em termos dos duais não-torcidos pelas equações (1.43) e (1.44), observamos que as fórmulas

$$
\begin{aligned}
& \Phi_{\vec{J}^{\oplus} E}=\Phi_{\vec{J}^{*} E} \otimes \Phi_{M}^{-1 *}, \\
& \Phi_{J^{\oplus} E}=\Phi_{J^{\star} E} \otimes \Phi_{M}^{-1 *},
\end{aligned}
$$

estabelecem correspondências biunívocas entre os automorfismos de $\vec{J}^{\otimes} E$ e de $\vec{J}^{*} E$, assim como entre os automorfismos de $J^{\circledast} E$ e de $J^{\star} E$. Escolhendo uma forma de volume $\epsilon$ no espaço-tempo $M$ e fazendo uso dos isomorfismos $\vec{J}^{\otimes} E \cong \vec{J}^{*} E$ e $J^{\oplus} E \cong J^{\star} E$ por ela induzidos, podemos identificar seções $\mathcal{H}_{0}$ de $J^{\star} E$ sobre $\vec{J}^{*} E$ com seções $\mathcal{H}$ de $J^{\otimes} E$ sobre $\vec{J} \circledast E$. Assim, chegamos à seguinte 
Proposição 1.2 Dados uma hamiltoniana $\mathcal{H}$ e um automorfismo $\Phi_{\vec{J}^{\oplus} E}: \vec{J}^{\circledast} E \rightarrow \vec{J} \vec{J}^{\circledast}$ de $\vec{J}{ }^{\circledast} E$ sobre o difeomorfismo $\Phi_{M}: M \rightarrow M$ de $M$, existe um único automorfismo $\Phi_{J^{\oplus E}}: J^{\oplus E} \rightarrow J^{\oplus E}$ de $J^{\oplus E}$ sobre o mesmo difeomorfismo $\Phi_{M}: M \rightarrow M$ de $M$ com as seguintes propriedades:

- $\Phi_{J^{\oplus} E}$ projeta sobre $\Phi_{\vec{J}^{\oplus} E}$ sob a projeção $\eta$, i.e.,

$$
\eta \circ \Phi_{J^{\oplus E}}=\Phi_{\vec{J} \oplus E} \circ \eta
$$

- $\Phi_{J^{\oplus E}}$ satisfaz a seguinte condição de "equivariância torcida" em relação à ação (1.102) de $\mathbb{R}$, para qualquer escolha de $\epsilon$ :

$$
\begin{gathered}
\Phi_{J^{\star} E}(z+\alpha \epsilon)=\Phi_{J^{\star} E}(z)+\alpha \operatorname{det}^{-1}\left(\Phi_{M}^{\prime}(m)\right) \epsilon \\
\text { para } z \in J^{\star} E \text { sobre } m \in M, \alpha \in \mathbb{R} .
\end{gathered}
$$

- $\Phi_{J^{\oplus} E}$ é um levantamento de $\Phi_{\vec{J}^{\oplus} E}$ pela hamiltoniana $\mathcal{H}$, i.e.,

$$
\Phi_{J^{\oplus E}} \circ \mathcal{H}=\mathcal{H} \circ \Phi_{\vec{J} \oplus E}
$$

Chamaremos $\Phi_{J^{\oplus} E}$ o levantamento hamiltoniano de $\Phi_{\vec{J}_{E} E}$.

A versão infinitesimal é a seguinte:

Proposição 1.3 Dados uma hamiltoniana $\mathcal{H}$ e um campo vetorial $X_{\vec{J}^{\oplus} E}$ sobre $\vec{J}^{\circledast} E$ que é projetável sobre um campo vetorial $X_{M}$ sobre $M$, existe um único campo vetorial $X_{J \oplus_{E}}$ sobre $J^{\oplus} E$ que é projetável sobre o mesmo campo vetorial $X_{M}$ sobre $M$ com as seguintes propriedades:

- $X_{J^{\oplus} E}$ projeta sobre $X_{\vec{J}^{\oplus E}}$ sob a projeção $\eta$, i.e.,

$$
T \eta \circ X_{J^{\oplus} E}=X_{\vec{J}^{\oplus} E} \circ \eta \text {. }
$$

- $X_{J^{\oplus}}$ satisfaz a seguinte condição de "equivariância torcida" em relação ao campo fundamental $\Sigma_{\epsilon}$, para qualquer escolha de $\epsilon$ :

$$
\left[X_{J \oplus E}, \Sigma_{\epsilon}\right]=\operatorname{div} X_{M} \Sigma_{\epsilon}
$$

- $X_{J^{\oplus E}}$ é um levantamento de $X_{\vec{J}_{E}}$ pela hamiltoniana $\mathcal{H}$, i.e.,

$$
X_{J \oplus E} \circ \mathcal{H}=T \mathcal{H} \circ X_{\vec{J}^{\circledast E}} .
$$


Chamaremos $X_{J^{\oplus E}}$ o levantamento hamiltoniano de $X_{\vec{J}_{\oplus} E}$. Em coordenadas locais adaptadas $\left(x^{\mu}, q^{i}, p_{i}^{\mu}\right)$ para $\vec{J}^{\circledast} E e\left(x^{\mu}, q^{i}, p_{i}^{\mu}, p\right)$ para $J^{\circledast} E$, escrevendo $\mathcal{H}=-H d^{n} x$ e

$$
X_{\vec{J}^{\oplus} E}=X^{\mu} \frac{\partial}{\partial x^{\mu}}+X^{i} \frac{\partial}{\partial q^{i}}+X_{i}^{\mu} \frac{\partial}{\partial p_{i}^{\mu}},
$$

obtemos

$$
\begin{aligned}
X_{J^{\oplus} E}= & X^{\mu} \frac{\partial}{\partial x^{\mu}}+X^{i} \frac{\partial}{\partial q^{i}}+X_{i}^{\mu} \frac{\partial}{\partial p_{i}^{\mu}} \\
& -\left((p+H) \frac{\partial X^{\mu}}{\partial x^{\mu}}+\frac{\partial H}{\partial x^{\mu}} X^{\mu}+\frac{\partial H}{\partial q^{i}} X^{i}+\frac{\partial H}{\partial p_{i}^{\mu}} X_{i}^{\mu}\right) \frac{\partial}{\partial p} .
\end{aligned}
$$

DemonstraÇÃo. Em termos de coordenadas locais adaptadas $\left(x^{\mu}, q^{i}, p_{i}^{\mu}\right)$ para $\vec{J}{ }^{\otimes} E$ e $\left(x^{\mu}, q^{i}, p_{i}^{\mu}, p\right)$ para $J^{\oplus E}$, campos vetoriais $X_{\vec{J}^{\oplus E}}$ são escritos na forma

$$
X_{\vec{J}^{\circledR} E}=\vec{X}^{\mu} \frac{\partial}{\partial x^{\mu}}+\vec{X}^{i} \frac{\partial}{\partial q^{i}}+\vec{X}_{i}^{\mu} \frac{\partial}{\partial p_{i}^{\mu}},
$$

onde os coeficientes $\vec{X}^{\mu}, \vec{X}^{i}$ e $\vec{X}_{i}^{\mu}$ são funções gerais das variáveis $x^{\rho}, q^{r}$ e $p_{r}^{\rho}$, enquanto que campos vetoriais $X_{J{ }^{\Theta_{E}}}$ são escritos na forma

$$
X_{J^{\oplus E}}=X^{\mu} \frac{\partial}{\partial x^{\mu}}+X^{i} \frac{\partial}{\partial q^{i}}+X_{i}^{\mu} \frac{\partial}{\partial p_{i}^{\mu}}+X_{0} \frac{\partial}{\partial p},
$$

onde os coeficientes $X^{\mu}, X^{i}, X_{i}^{\mu}$ e $X_{0}$ são funções gerais das variáveis $x^{\rho}, q^{r}, p_{r}^{\rho}$ e $p$. Nestes termos, o fato de que o segundo projeta sobre o primeiro significa que

$$
X^{\mu}=\vec{X}^{\mu}, X^{i}=\vec{X}^{i}, X_{i}^{\mu}=\vec{X}_{i}^{\mu} .
$$

Em particular, os coeficientes $X^{\mu}, X^{i}$ e $X_{i}^{\mu}$ independem de $p$. Calculando o colchete de Lie, temos

$$
\begin{aligned}
{\left[X_{J^{\oplus E}}, \Sigma_{\epsilon}\right] } & =\left[X^{\mu} \frac{\partial}{\partial x^{\mu}}+X^{i} \frac{\partial}{\partial q^{i}}+X_{i}^{\mu} \frac{\partial}{\partial p_{i}^{\mu}}+X_{0} \frac{\partial}{\partial p}, \epsilon_{0} \frac{\partial}{\partial p}\right] \\
& =\left(X^{\mu} \frac{\partial \epsilon_{0}}{\partial x^{\mu}}-\epsilon_{0} \frac{\partial X_{0}}{\partial p}\right) \frac{\partial}{\partial p} .
\end{aligned}
$$

Por outro lado, pela definição da divergência,

$$
\operatorname{div} X_{M} \epsilon=L_{X_{M}} \epsilon
$$


ou em coordenadas,

$$
\operatorname{div} X_{M}=X^{\mu} \frac{1}{\epsilon_{0}} \frac{\partial \epsilon_{0}}{\partial x^{\mu}}+\frac{\partial X^{\mu}}{\partial x^{\mu}} .
$$

Portanto, a condição (1.113) é equivalente à relação

$$
\frac{\partial X_{0}}{\partial p}=-\frac{\partial X^{\mu}}{\partial x^{\mu}}
$$

que pode ser integrada como segue:

$$
X_{0}=-p \frac{\partial X^{\mu}}{\partial x^{\mu}}+Y_{0}
$$

onde $Y_{0}$ independe da variável $p$. Finalmente, a propriedade de $X_{J^{\oplus} E}$ ser um levantamento de $X_{\vec{J}^{\oplus} E}$ pela hamiltoniana $\mathcal{H}$ significa que

$$
\left.X_{0}\right|_{p=-H}=-\left(\frac{\partial H}{\partial x^{\mu}} X^{\mu}+\frac{\partial H}{\partial q^{i}} X^{i}+\frac{\partial H}{\partial p_{i}^{\mu}} X_{i}^{\mu}\right) .
$$

Combinando as últimas duas equações, obtemos a equação (1.116).

Observemos que o levantamento hamiltoniano providencia um método de identificar automorfismos no espaço de multifase comum e no espaço de multifase estendido. Entre eles, podemos distinguir aqueles que preservam a forma multissimplética $\omega$ e que parecem ser os candidatos naturais para definir o conceito de uma transformação multicanônica, que seria o análogo natural da noção de uma transformação canônica no formalismo simplético da mecânica.

Finalmente, a ação de automorfismos e automorfismos infinitesimais relacionados por levantamento hamiltoniano sobre seções é simples. Em particular, quando comparamos a ação de um automorfismo infinitesimal $X=X_{\vec{J}_{E}}$ sobre uma seção $\phi=(\varphi, \pi)$ de $\vec{J}^{\circledast} E$ com a ação do seu levantamento hamiltoniano $\tilde{X}=X_{J^{\oplus} E}$ sobre a seção composta $\tilde{\phi}=\mathcal{H}(\phi)=(\varphi, \pi,-H(\varphi, \pi))$ de $J^{\circledast} E$, obtemos

$$
\delta_{X} \phi=\left(\delta_{X} \varphi, \delta_{X} \pi\right) \quad, \quad \delta_{\tilde{X}} \tilde{\phi}=\left(\delta_{\tilde{X}} \varphi, \delta_{\tilde{X}} \pi,-\delta_{\tilde{X}} H(\varphi, \pi)\right),
$$

com

$$
\begin{aligned}
\delta_{\tilde{X}} \varphi^{i} & =\delta_{X} \varphi^{i}=X^{i}(\varphi)-\partial_{\mu} \varphi^{i} X^{\mu} \\
\delta_{\tilde{X}} \pi_{i}^{\mu}= & \delta_{X} \pi_{i}^{\mu}=X_{i}^{\mu}(\varphi, \pi)-\partial_{\nu} \pi_{i}^{\mu} X^{\nu}, \\
\delta_{\tilde{X}} H(\varphi, \pi)= & \frac{\partial H}{\partial q^{i}}(\varphi, \pi)\left(X^{i}(\varphi)-\partial_{\mu} \varphi^{i} X^{\mu}\right) \\
& +\frac{\partial H}{\partial p_{i}^{\mu}}(\varphi, \pi)\left(X_{i}^{\mu}(\varphi, \pi)-\partial_{\nu} \pi_{i}^{\mu} X^{\nu}\right) .
\end{aligned}
$$


Observemos as diferenças entre os dois processos de levantamento canônico e de levantamento hamiltoniano: Ambos partem de um automorfismo de $\vec{J}^{\circledR} E$ que induz um automorfismo de $E$, onde no caso do levantamento canônico o primeiro é obtido por levantamento do segundo (correspondendo a um campo vetorial sobre $\vec{J}^{\circledast} E$ com coeficientes $X_{i}^{\mu}$ determinados pelos coeficientes $X^{i}$ e $\left.X^{\mu}\right)$, enquanto que no caso do levantamento hamiltoniano o primeiro é independente do segundo (correspondendo a um campo vetorial sobre $\vec{J}^{\otimes} E$ com coeficientes $X_{i}^{\mu}$ independentes dos coeficientes $X^{i}$ e $\left.X^{\mu}\right)$. Um melhor entendimento da questão quando estes. dois levantamentos coincidem será obtido mais adiante.

\subsection{Campos vetoriais hamiltonianos}

Em variedades multissimpléticas, temos a mesma noção de campos vetoriais hamiltonianos (localmente, globalmente ou exatamente) que em variedades simpléticas:

Definição 1.5 Seja $X \in \mathfrak{X}(P)$ um campo vetorial sobre uma variedade multissimplética $P$. O campo $X$ é dito localmente hamiltoniano se $i_{X} \omega$ for uma forma fechada, ou seja, se

$$
L_{X} \omega=0
$$

o que significa que o fluxo de $X$ preserva a forma multissimplética $\omega$, e é dito hamiltoniano se $i_{X} \omega$ for uma forma exata, ou seja, se existir uma $(n-1)$-forma $f$ tal que

$$
i_{X} \omega=d f .
$$

Neste caso, $X$ é unicamente determinado por $f$ (pois $\omega$ é não-degenerada) e chamado o campo hamiltoniano associado a $f$, denotado por $X_{f}$. Reciprocamente, se para uma dada $(n-1)$-forma $f$ existir um campo vetorial $X$ tal que vale a fórmula (1.124), então dizemos que $f$ é uma $(n-1)$-forma hamiltoniana e que $f$ é associada a $X$.

A novidade na geometria multissimplética é que $f$ é uma $(n-1)$-forma, ao invés de ser simplesmente uma função, e que nem todas as $(n-1)$-formas são hamiltonianas: Ocorre que a condição (1.124) impõe fortes restrições não apenas sobre $X$ mas também sobre $f$.

No caso em que a variedade for exatamente multissimplética, podemos completar a definição acima como segue:

Definição $1.6 \quad$ Seja $X \in \mathfrak{X}(P)$ um campo vetorial sobre uma variedade exatamente multissimplética $P$. $O$ campo $X$ é dito exatamente hamiltoniano se

$$
L_{X} \theta=0
$$

o que significa que o fluxo de $X$ preserva a forma multicanônica $\theta$. 
É fácil ver que vale

$$
X \text { exatamente hamiltoniano } \Longrightarrow X \text { hamiltoniano, }
$$

$\mathrm{e}$

$$
X \text { hamiltoniano } \Longrightarrow X \text { localmente hamiltoniano . }
$$

De fato, a primeira implicação segue de

Proposição 1.4 Seja $X \in \mathfrak{X}(P)$ um campo vetorial exatamente hamiltoniano sobre uma variedade exatamente multissimplética $P$. Então

$$
J(X)=i_{X} \theta
$$

é uma $(n-1)$-forma hamiltoniana cujo campo hamiltoniano associado é o próprio $X$ :

$$
X_{J(X)}=X .
$$

DemonstraÇão. É um cálculo simples:

$$
d(J(X))=d\left(i_{X} \theta\right)=L_{X} \theta-i_{X} d \theta=i_{X} \omega .
$$

\subsubsection{Espaço de multifase estendido}

No caso do espaço de multifase estendido $J^{\circledast} E$, onde o núcleo de $\theta$ contém o núcleo de $\omega$ e onde podemos lançar mão do campo de Euler $\Sigma$ para obter $\theta$ a partir de $\omega$, conforme a equação (1.99), este constitui uma ferramenta chave para a completa classificação dos campos vetoriais localmente hamiltonianos, através da sua decomposição em componentes homogêneos sob a derivada de Lie ao longo de $\Sigma$. A seguir, apresentamos a classificação dos campos multivetoriais exatamente hamiltonianos e dos campos multivetoriais localmente hamiltonianos sobre $J^{\oplus} E$ obtida em [13] para o caso especial e mais simples de campos vetoriais.

Como primeiro passo, formulamos duas afirmações simples que generalizam a proposição anterior:

Proposição 1.5 Seja $X \in \mathfrak{X}\left(J^{\oplus} E\right)$ um campo vetorial localmente hamiltoniano sobre o espaço de multifase estendido $J^{\oplus} E$. Então

$$
J(X)=i_{X} \theta
$$


é uma $(n-1)$-forma hamiltoniana cujo campo hamiltoniano associado é $X+[\Sigma, X]$ :

$$
X_{J(X)}=X+[\Sigma, X]
$$

DEMONSTRAÇÃo. É um cálculo simples:

$$
\begin{aligned}
d(J(X)) & =d\left(i_{X} \theta\right)=L_{X} \theta-i_{X} d \theta \\
& =-L_{X} i_{\Sigma} \omega+i_{X} \omega \\
& =-L_{X} i_{\Sigma} \omega+i_{\Sigma} L_{X} \omega+i_{X} \omega \\
& =-i_{[X, \Sigma]} \omega+i_{X} \omega .
\end{aligned}
$$

Proposição 1.6 Seja $X \in \mathfrak{X}\left(J^{\otimes} E\right)$ um campo vetorial localmente hamiltoniano sobre o espaço de multifase estendido $J^{\otimes} E$. Então $X$ é homogêneo de grau 0 sob $L_{\Sigma}$ se e somente se $X$ for exatamente hamiltoniano.

DEMONSTRAÇÃo. A afirmação segue imediatamente da igualdade

$$
L_{X} \theta=i_{[\Sigma, X]} \omega
$$

que é válida desde que $L_{X} \omega=0$, em conjunto com o fato de que $\omega$ é não-degenerada.

Para prosseguir, usamos coordenadas locais adaptadas, em termos das quais um campo vetorial $X$ qualquer sobre $J^{\circledast} E$ assume a forma

$$
X=X^{\mu} \frac{\partial}{\partial x^{\mu}}+X^{i} \frac{\partial}{\partial q^{i}}+X_{i}^{\mu} \frac{\partial}{\partial p_{i}^{\mu}}+X_{0} \frac{\partial}{\partial p},
$$

onde todos os coeficientes $X^{\mu}, X^{i}, X_{i}^{\mu}$ e $X_{0}$ são funções (em geral arbitrárias) das variáveis $x^{\kappa}, q^{k}, p_{k}^{\kappa}$ e $p$. Tendo em vista as formas explícitas (1.82) de $\omega$ e (1.81) de $\theta$ em coordenadas locais adaptadas, torna-se óbvio que

$$
\begin{aligned}
i_{X} \omega= & X^{\nu} d q^{i} \wedge d p_{i}^{\mu} \wedge d^{n} x_{\mu \nu}-X_{i}^{\mu} d q^{i} \wedge d^{n} x_{\mu}+X^{i} d p_{i}^{\mu} \wedge d^{n} x_{\mu} \\
& +X^{\mu} d p \wedge d^{n} x_{\mu}-X_{0} d^{n} x
\end{aligned}
$$

e

$$
i_{X} \theta=\left(p_{i}^{\mu} X^{i}+p X^{\mu}\right) d^{n} x_{\mu}-p_{i}^{\mu} X^{\nu} d q^{i} \wedge d^{n} x_{\mu \nu} .
$$

Estas fórmulas constituem o ponto de partida para a demonstração do seguinte teorema. 
Teorema 1.1 Seja $X \in \mathfrak{X}\left(J^{\circledast E} E\right)$ um campo vetorial exatamente hamiltoniano sobre o espaço de multifase estendido $J^{\circledast E}$. Então em coordenadas locais adaptadas, as componentes de $X$ conforme definidas pela equação (1.133) acima estão sujeitas às seguintes restrições:

- Os coeficientes $X^{\mu}$ independem das variáveis $p$ e $p_{k}^{\kappa}$. Para $N>1$, também independem das variáveis $q^{k}$.

- Os coeficientes $X^{i}$ independem das variáveis $p$ e $p_{k}^{\kappa}$.

- Os coeficientes $X_{i}^{\mu}$ e $X_{0}$ são definidos em termos dos coeficientes $X^{\mu}$ e $X^{i}$ pelas seguintes fórmulas:

$$
\begin{gathered}
X_{i}^{\mu}=-p \frac{\partial X^{\mu}}{\partial q^{i}}-p_{j}^{\mu} \frac{\partial X^{j}}{\partial q^{i}}+p_{i}^{\nu} \frac{\partial X^{\mu}}{\partial x^{\nu}}-p_{i}^{\mu} \frac{\partial X^{\nu}}{\partial x^{\nu}} \\
X_{0}=-p \frac{\partial X^{\mu}}{\partial x^{\mu}}-p_{i}^{\mu} \frac{\partial X^{i}}{\partial x^{\mu}}
\end{gathered}
$$

DemonstraÇÃo. Seja $X \in \mathfrak{X}\left(J^{\circledast} E\right)$ um campo vetorial qualquer sobre $J^{\circledast} E$. Em coordenadas locais adaptadas, aplicamos a derivada exterior $d$ à equação (1.135) para obter

$$
\begin{aligned}
d\left(i_{X} \theta\right)= & X^{i} d p_{i}^{\mu} \wedge d^{n} x_{\mu}+\frac{\partial X^{i}}{\partial x^{\mu}} p_{i}^{\mu} d^{n} x+\frac{\partial X^{i}}{\partial q^{j}} p_{i}^{\mu} d q^{j} \wedge d^{n} x_{\mu} \\
& +\frac{\partial X^{i}}{\partial p_{j}^{\nu}} p_{i}^{\mu} d p_{j}^{\nu} \wedge d^{n} x_{\mu}+\frac{\partial X^{i}}{\partial p} p_{i}^{\mu} d p \wedge d^{n} x_{\mu} \\
& +X^{\mu} d p \wedge d^{n} x_{\mu}+\frac{\partial X^{\mu}}{\partial x^{\mu}} p d^{n} x+\frac{\partial X^{\mu}}{\partial q^{i}} p d q^{i} \wedge d^{n} x_{\mu} \\
& +\frac{\partial X^{\mu}}{\partial p_{j}^{\nu}} p d p_{j}^{\nu} \wedge d^{n} x_{\mu}+\frac{\partial X^{\mu}}{\partial p} p d p \wedge d^{n} x_{\mu} \\
& -X^{\nu} d p_{i}^{\mu} \wedge d q^{i} \wedge d^{n} x_{\mu \nu}+\frac{\partial X^{\nu}}{\partial x^{\kappa}} p_{i}^{\mu} d q^{i} \wedge d x^{\kappa} \wedge d^{n} x_{\mu \nu} \\
& -\frac{\partial X^{\nu}}{\partial q^{j}} p_{i}^{\mu} d q^{j} \wedge d q^{i} \wedge d^{n} x_{\mu \nu}-\frac{\partial X^{\nu}}{\partial p_{k}^{\kappa}} p_{i}^{\mu} d p_{k}^{\kappa} \wedge d q^{i} \wedge d^{n} x_{\mu \nu} \\
& -\frac{\partial X^{\nu}}{\partial p} p_{i}^{\mu} d p \wedge d q^{i} \wedge d^{n} x_{\mu \nu} .
\end{aligned}
$$

Assim, pela equação (1.134), 


$$
\begin{aligned}
L_{X} \theta= & d\left(i_{X} \theta\right)-i_{X} \omega \\
= & \left(\frac{\partial X^{\mu}}{\partial x^{\mu}} p+\frac{\partial X^{i}}{\partial x^{\mu}} p_{i}^{\mu}+X_{0}\right) d^{n} x \\
& +\left(\frac{\partial X^{j}}{\partial q^{i}} p_{j}^{\mu}-\frac{\partial X^{\mu}}{\partial x^{\nu}} p_{i}^{\nu}+\frac{\partial X^{\nu}}{\partial x^{\nu}} p_{i}^{\mu}+\frac{\partial X^{\mu}}{\partial q^{i}} p+X_{i}^{\mu}\right) d q^{i} \wedge d^{n} x_{\mu} \\
& +\left(\frac{\partial X^{i}}{\partial p_{j}^{\nu}} p_{i}^{\mu}+\frac{\partial X^{\mu}}{\partial p_{j}^{\nu}} p\right) d p_{j}^{\nu} \wedge d^{n} x_{\mu} \\
& +\left(\frac{\partial X^{i}}{\partial p} p_{i}^{\mu}+\frac{\partial X^{\mu}}{\partial p} p\right) d p \wedge d^{n} x_{\mu} \\
& +\frac{\partial X^{\nu}}{\partial q^{j}} p_{i}^{\mu} d q^{i} \wedge d q^{j} \wedge d^{n} x_{\mu \nu} \\
& +\frac{\partial X^{\nu}}{\partial p_{k}^{\kappa}} p_{i}^{\mu} d q^{i} \wedge d p_{k}^{\kappa} \wedge d^{n} x_{\mu \nu} \\
& +\frac{\partial X^{\nu}}{\partial p} p_{i}^{\mu} d q^{i} \wedge d p \wedge d^{n} x_{\mu \nu} .
\end{aligned}
$$

Numerando os termos nesta equação de 1 a 7 , a condição

$$
L_{X} \theta=0
$$

leva às seguintes conclusões:

- Termo 7: Este se anula se e somente se os coeficientes $X^{\mu}$ independem da variável $p$, e então o quarto termo se anula se e somente se os coeficientes $X^{i}$ independem da variável $p$.

- Termo 6: Este se anula se e somente se os coeficientes $X^{\mu}$ independem das variáveis $p_{k}^{\kappa}$, e então o terceiro termo se anula se e somente se os coeficientes $X^{i}$ independem das variáveis $p_{k}^{\kappa}$.

- Termo 5: Este se anula se e somente se os coeficientes $X^{\mu}$ independem das variáveis $q^{k}$, caso $N>1$. No caso $N=1$, este termo se anula identicamente.

- Termos 1 e 2: Estes se anulam se e somente se os coeficientes $X_{i}^{\mu}$ e $X_{0}$ são definidos em termos dos coeficientes $X^{\mu}$ e $X^{i}$ segundo as equações (1.136) e (1.137). 
Passando a campos vetoriais localmente mas não necessariamente exatamente hamiltonianos, temos o seguinte teorema.

Teorema 1.2 Seja $X \in \mathfrak{X}\left(J^{\otimes} E\right)$ um campo vetorial localmente hamiltoniano sobre o espaço de multifase estendido $J^{\circledast} E$. Então $\mathrm{em}$ coordenadas locais adaptadas, as componentes de $X$ conforme definidas pela equação (1.133) acima estão sujeitas às seguintes restrições:

- Os coeficientes $X^{\mu}$ independem das variáveis $p$ e $p_{k}^{\kappa}$. Para $N>1$, também independem das variáveis $q^{k}$.

- Os coeficientes $X^{i}$ independem das variáveis $p$ e $p_{k}^{\kappa}$.

- Os coeficientes $X_{i}^{\mu}$ e $X_{0}$ são definidos em termos dos coeficientes $X^{\mu}$ e $X^{i}$, em conjunto com novos coeficientes $X_{-}^{\mu}$ que também independem das variáveis $p$ e $p_{k}^{\kappa}$, pelas seguintes fórmulas:

$$
\begin{gathered}
X_{i}^{\mu}=-p \frac{\partial X^{\mu}}{\partial q^{i}}-p_{j}^{\mu} \frac{\partial X^{j}}{\partial q^{i}}+p_{i}^{\nu} \frac{\partial X^{\mu}}{\partial x^{\nu}}-p_{i}^{\mu} \frac{\partial X^{\nu}}{\partial x^{\nu}}+\frac{\partial X_{-}^{\mu}}{\partial q^{i}} \\
X_{0}=-p \frac{\partial X^{\mu}}{\partial x^{\mu}}-p_{i}^{\mu} \frac{\partial X^{i}}{\partial x^{\mu}}+\frac{\partial X_{-}^{\mu}}{\partial x^{\mu}} .
\end{gathered}
$$

DemonstraÇão. Seja $X \in \mathfrak{X}\left(J^{\circledast} E\right)$ um campo vetorial qualquer sobre $J^{\circledast} E$. Em coordenadas locais adaptadas, aplicamos a derivada exterior $d$ à equação (1.134) para obter

$$
\begin{aligned}
L_{X} \omega= & d\left(i_{X} \omega\right) \\
= & \frac{\partial X^{\nu}}{\partial x^{\kappa}} d q^{i} \wedge d p_{i}^{\mu} \wedge d x^{\kappa} \wedge d^{n} x_{\mu \nu}+\frac{\partial X^{\nu}}{\partial q^{j}} d q^{j} \wedge d q^{i} \wedge d p_{i}^{\mu} \wedge d^{n} x_{\mu \nu} \\
& +\frac{\partial X^{\nu}}{\partial p_{k}^{\kappa}} d p_{k}^{\kappa} \wedge d q^{i} \wedge d p_{i}^{\mu} \wedge d^{n} x_{\mu \nu}+\frac{\partial X^{\nu}}{\partial p} d p \wedge d q^{i} \wedge d p_{i}^{\mu} \wedge d^{n} x_{\mu \nu} \\
& +\frac{\partial X_{i}^{\mu}}{\partial x^{\mu}} d q^{i} \wedge d^{n} x-\frac{\partial X_{i}^{\mu}}{\partial q^{j}} d q^{j} \wedge d q^{i} \wedge d^{n} x_{\mu} \\
& -\frac{\partial X_{i}^{\mu}}{\partial p_{j}^{\nu}} d p_{j}^{\nu} \wedge d q^{i} \wedge d^{n} x_{\mu}-\frac{\partial X_{i}^{\mu}}{\partial p} d p \wedge d q^{i} \wedge d^{n} x_{\mu}
\end{aligned}
$$




$$
\begin{aligned}
& -\frac{\partial X^{i}}{\partial x^{\mu}} d p_{i}^{\mu} \wedge d^{n} x+\frac{\partial X^{i}}{\partial q^{j}} d q^{j} \wedge d p_{i}^{\mu} \wedge d^{n} x_{\mu} \\
& +\frac{\partial X^{i}}{\partial p_{j}^{\nu}} d p_{j}^{\nu} \wedge d p_{i}^{\mu} \wedge d^{n} x_{\mu}+\frac{\partial X^{i}}{\partial p} d p \wedge d p_{i}^{\mu} \wedge d^{n} x_{\mu} \\
& -\frac{\partial X^{\mu}}{\partial x^{\mu}} d p \wedge d^{n} x+\frac{\partial X^{\mu}}{\partial q^{i}} d q^{i} \wedge d p \wedge d^{n} x_{\mu}+\frac{\partial X^{\mu}}{\partial p_{j}^{\nu}} d p_{j}^{\nu} \wedge d p \wedge d^{n} x_{\mu} \\
& -\frac{\partial X_{0}}{\partial q^{i}} d q^{i} \wedge d^{n} x-\frac{\partial X_{0}}{\partial p_{i}^{\mu}} d p_{i}^{\mu} \wedge d^{n} x-\frac{\partial X_{0}}{\partial p} d p \wedge d^{n} x .
\end{aligned}
$$

Reagrupando os termos, obtemos

$$
\begin{aligned}
L_{X} \omega= & \left(\frac{\partial X_{i}^{\mu}}{\partial x^{\mu}}-\frac{\partial X_{0}}{\partial q^{i}}\right) d q^{i} \wedge d^{n} x \\
& -\left(\frac{\partial X^{i}}{\partial x^{\mu}}+\frac{\partial X_{0}}{\partial p_{i}^{\mu}}\right) d p_{i}^{\mu} \wedge d^{n} x \\
& -\left(\frac{\partial X^{\mu}}{\partial x^{\mu}}+\frac{\partial X_{0}}{\partial p}\right) d p \wedge d^{n} x \\
& +\left(\frac{\partial X_{i}^{\mu}}{\partial p}+\frac{\partial X^{\mu}}{\partial q^{i}}\right) d q^{i} \wedge d p \wedge d^{n} x_{\mu} \\
& +\left(\frac{\partial X^{\sigma}}{\partial p_{k}^{\kappa}}-\delta_{\kappa}^{\sigma} \frac{\partial X^{k}}{\partial p}\right) d p_{k}^{\kappa} \wedge d p \wedge d^{n} x_{\sigma} \\
& +\left(\frac{\partial X_{i}^{\mu}}{\partial p_{j}^{\nu}}+\delta_{\nu}^{\mu} \frac{\partial X^{j}}{\partial q^{i}}-\delta_{i}^{j} \frac{\partial X^{\mu}}{\partial x^{\nu}}+\delta_{\nu}^{\mu} \delta_{i}^{j} \frac{\partial X^{\kappa}}{\partial x^{\kappa}}\right) d q^{i} \wedge d p_{j}^{\nu} \wedge d^{n} x_{\mu} \\
& +\frac{\partial X_{i}^{\mu}}{\partial q^{j}} d q^{i} \wedge d q^{j} \wedge d^{n} x_{\mu} \\
& +\frac{\partial X^{l}}{\partial p_{k}^{\kappa}} d p_{k}^{\kappa} \wedge d p_{l}^{\lambda} \wedge d^{n} x_{\lambda} \\
& +\frac{\partial X^{\sigma}}{\partial q^{k}} d q^{k} \wedge d q^{l} \wedge d p_{l}^{\lambda} \wedge d^{n} x_{\lambda \sigma} \\
& +\frac{\partial X^{\sigma}}{\partial p_{k}^{\kappa}} d q^{l} \wedge d p_{k}^{\kappa} \wedge d p_{l}^{\lambda} \wedge d^{n} x_{\lambda \sigma} \\
& +\frac{\partial X^{\nu}}{\partial p} d q^{i} \wedge d p_{i}^{\mu} \wedge d p \wedge d^{n} x_{\mu \nu}
\end{aligned}
$$


Numerando os termos nesta equação de 1 a 11 , a condição

$$
L_{X} \omega=0
$$

leva às seguintes conclusões:

- Termo 11: Este se anula se e somente se os coeficientes $X^{\mu}$ independem da variável $p$.

- Termo 10: Fixando índices $i, j, \mu, \nu, \rho$ e índices mutuamente diferentes $\rho_{1}, \ldots, \rho_{n-2}$, contraímos $L_{X} \omega$ com o campo multivetorial $\partial_{j} \wedge \partial_{\mu}^{i} \wedge \partial_{\nu}^{j} \wedge \partial_{\rho_{1}} \wedge \ldots \wedge \partial_{\rho_{n-2}}$ (sem soma sobre $j$ ), obtendo a relação

$$
\left.\frac{\partial X^{\sigma}}{\partial p_{i}^{\mu}} \epsilon_{\nu \sigma \rho_{1} \ldots \rho_{n-2}}=\delta_{j}^{i} \frac{\partial X^{\sigma}}{\partial p_{j}^{\nu}} \epsilon_{\mu \sigma \rho_{1} \ldots \rho_{n-2}} \quad \text { (sem soma sobre } j\right) .
$$

Agora, se inicialmente, fixamos apenas os índices $i, \mu$ e $\rho$, podemos sempre escolher os demais índices tais que $\nu, \rho, \rho_{1}, \ldots, \rho_{n-2}$ são mutuamente diferentes, o que reduz o lado esquerdo desta equação à expressão $\pm \partial X^{\rho} / \partial p_{i}^{\mu}$, enquanto que o lado direito se anula se escolhermos $j \neq i$ ou $\rho_{1}, \ldots, \rho_{n-2}$ tais que $\mu$ ocorre entre eles. Assim, concluímos que

$$
\frac{\partial X^{\rho}}{\partial p_{i}^{\mu}}=0
$$

exceto quando $N=1$ e $n>2$ e $\mu=\rho$ ou quando $N=1$ e $n=2$. Observe que quando $N=1$ e $\mu=\rho$, a equação anterior assume a forma ${ }^{5}$

$$
\left.\frac{\partial X^{\mu}}{\partial p^{\mu}} \epsilon_{\nu \mu \rho_{1} \ldots \rho_{n-2}}=\frac{\partial X^{\nu}}{\partial p^{\nu}} \epsilon_{\mu \nu \rho_{1} \ldots \rho_{n-2}} \quad \text { (sem soma sobre } \mu \text { e } \nu\right)
$$

o que implica

$$
\frac{\partial X^{\mu}}{\partial p^{\mu}}=-\frac{\partial X^{\kappa}}{\partial p^{\kappa}}=\frac{\partial X^{\lambda}}{\partial p^{\lambda}}=-\frac{\partial X^{\mu}}{\partial p^{\mu}} \quad(\text { sem soma sobre } \mu, \kappa \mathrm{e} \lambda)
$$

desde que $\mu, \kappa, \lambda$ são mutuamente diferentes. Portanto, a conclusão anterior, de que

$$
\frac{\partial X^{\rho}}{\partial p_{i}^{\mu}}=0
$$

vale mesmo quando $N=1$ e $n>2$ e $\mu=\rho$. A única exceção ocorre no caso em que $N=1$ e $n=2$, onde é apenas possível concluir que

$$
\frac{\partial X^{\mu}}{\partial p^{\mu}}=0
$$

No entanto, esta situação será coberta no próximo item.

\footnotetext{
${ }^{5}$ Quando $N=1$, omitimos os índices $i, j$, etc.
} 
- Termo 5: Fixando índices $i, \mu, \rho$ e $\rho_{1}, \ldots, \rho_{n-1}$ tais que os índices $\rho, \rho_{1}, \ldots, \rho_{n-1}$ são mutuamente diferentes, contraímos $L_{X} \omega$ com o campo multivetorial $\partial_{\mu}^{i} \wedge \partial_{0} \wedge \partial_{\rho_{1}} \wedge \ldots \wedge \partial_{\rho_{n-1}}$, obtendo a relação

$$
\frac{\partial X^{\rho}}{\partial p_{i}^{\mu}}=\delta_{\mu}^{\rho} \frac{\partial X^{i}}{\partial p} .
$$

Tomando o traço em $\mu$ e $\rho$ e usando o resultado do item anterior, chegamos à conclusão de que

$$
\frac{\partial X^{i}}{\partial p}=0
$$

mesmo no caso em que $N=1$ e $n=2$. Portanto, temos também

$$
\frac{\partial X^{\rho}}{\partial p_{i}^{\mu}}=0
$$

mesmo no caso em que $N=1$ e $n=2$.

- Termo 8: Fixando índices $i, j, \mu, \nu$ e índices mutuamente diferentes $\rho_{1}, \ldots, \rho_{n-1}$, contraímos $L_{X} \omega$ com o campo multivetorial $\partial_{\mu}^{i} \wedge \partial_{\nu}^{j} \wedge \partial_{\rho_{1}} \wedge \ldots \wedge \partial_{\rho_{n-1}}$, obtendo a relação

$$
\frac{\partial X^{j}}{\partial p_{i}^{\mu}} \epsilon_{\nu \rho_{1} \ldots \rho_{n-1}}=\frac{\partial X^{i}}{\partial p_{j}^{\nu}} \epsilon_{\mu \rho_{1} \ldots \rho_{n-1}} .
$$

Agora, se inicialmente, fixamos apenas os índices $i, j$ e $\mu$, podemos sempre escolher os demais índices tais que $\nu \neq \mu$ e que $\nu, \rho_{1}, \ldots, \rho_{n-1}$ são mutuamente diferentes, o que reduz o lado esquerdo desta equação à expressão $\pm \partial X^{j} / \partial p_{i}^{\mu}$, enquanto que o lado direito se anula. Assim, chegamos à conclusão de que

$$
\frac{\partial X^{j}}{\partial p_{i}^{\mu}}=0
$$

- Termo 9: Fixando índices $i, j, \rho$ e $\nu, \rho_{1}, \ldots, \rho_{n-2}$ tais que os índices $\nu, \rho, \rho_{1}, \ldots, \rho_{n-2}$ são mutuamente diferentes, contraímos $L_{X} \omega$ com o campo multivetorial $\partial_{i} \wedge \partial_{j} \wedge \partial_{\nu}^{j} \wedge \partial_{\rho_{1}} \wedge \ldots \wedge \partial_{\rho_{n-2}}$ (sem soma sobre $\left.j\right)$, obtendo a relação

$$
\left.\frac{\partial X^{\rho}}{\partial q^{i}}=\delta_{j}^{i} \frac{\partial X^{\rho}}{\partial q^{j}} \quad \text { (sem soma sobre } j\right) .
$$

Assim, chegamos à conclusão de que

$$
\frac{\partial X^{\rho}}{\partial q^{i}}=0,
$$

exceto no caso em que $N=1$ onde o termo inteiro se anula identicamente e nenhuma conclusão pode ser tirada. 
Para prosseguir, escrevemos as equações obtidas dos termos restantes.

- Termo 1:

$$
\frac{\partial X_{0}}{\partial q^{i}}=\frac{\partial X_{i}^{\mu}}{\partial x^{\mu}}
$$

- Termo 2:

$$
\frac{\partial X_{0}}{\partial p_{i}^{\mu}}=-\frac{\partial X^{i}}{\partial x^{\mu}}
$$

- Termo 3:

$$
\frac{\partial X_{0}}{\partial p}=-\frac{\partial X^{\mu}}{\partial x^{\mu}}
$$

- Termo 4:

$$
\frac{\partial X_{i}^{\mu}}{\partial p}=-\frac{\partial X^{\mu}}{\partial q^{i}}
$$

- Termo 6:

$$
\frac{\partial X_{i}^{\mu}}{\partial p_{j}^{\nu}}=-\delta_{\nu}^{\mu} \frac{\partial X^{j}}{\partial q^{i}}+\delta_{i}^{j} \frac{\partial X^{\mu}}{\partial x^{\nu}}-\delta_{\nu}^{\mu} \delta_{i}^{j} \frac{\partial X^{\kappa}}{\partial x^{\kappa}}
$$

- Termo 7:

$$
\frac{\partial X_{i}^{\mu}}{\partial q^{j}}=\frac{\partial X_{j}^{\mu}}{\partial q^{i}}
$$

Começando pelas equações (1.141) e (1.142), nota-se que o lado direito independe da variável $p$ e das variáveis $p_{k}^{\kappa}$; portanto elas podem ser integradas diretamente para concluir que

$$
X_{0}=-p \frac{\partial X^{\mu}}{\partial x^{\mu}}-p_{i}^{\mu} \frac{\partial X^{i}}{\partial x^{\mu}}+Y_{0}
$$

onde $Y_{0}$ é uma função local sobre $E$ : independe da variável $p$ e das variáveis $p_{k}^{\kappa}$. O mesmo argumento aplicado às equações (1.143) e (1.144) mostra que

$$
X_{i}^{\mu}=-p \frac{\partial X^{\mu}}{\partial q^{i}}-p_{j}^{\mu} \frac{\partial X^{j}}{\partial q^{i}}+p_{i}^{\nu} \frac{\partial X^{\mu}}{\partial x^{\nu}}-p_{i}^{\mu} \frac{\partial X^{\kappa}}{\partial x^{\kappa}}+Y_{i}^{\mu}
$$

onde os $Y_{i}^{\mu}$ são funções locais sobre $E$ : independem da variável $p$ e das variáveis $p_{k}^{\kappa}$. Substituindo estas relações nas equações (1.140) e (1.145), vem

$$
\frac{\partial Y_{0}}{\partial q^{i}}=\frac{\partial Y_{i}^{\mu}}{\partial x^{\mu}}
$$


$\mathrm{e}$

$$
\frac{\partial Y_{i}^{\mu}}{\partial q^{j}}=\frac{\partial Y_{j}^{\mu}}{\partial q^{i}},
$$

respectivamente. Finalmente, este último sistema de equações pode ser resolvido pondo

$$
Y_{0}=\frac{\partial X_{-}^{\mu}}{\partial x^{\mu}}, Y_{i}^{\mu}=\frac{\partial X_{-}^{\mu}}{\partial q^{i}},
$$

onde os $X_{-}^{\mu}$ são funções locais sobre $E$ : independem da variável $p$ e das variáveis $p_{k}^{\kappa}$. Isso demonstra as equações (1.138) e (1.139) e conclui a demonstração do teorema.

Para encontrar uma interpretação global das conclusões destes teoremas, observamos que os primeiros dois itens afirmam que um campo vetorial $X$ (exatamente ou localmente) hamiltoniano sobre $J^{\circledast} E$ é projetável sobre um campo vetorial $X_{E}$ sobre $E$ que, para $N>1$, por sua vez é projetável sobre um campo vetorial $X_{M}$ sobre $M$. Usando o levantamento canônico $X_{J^{\oplus} E}$ de $X_{E}$ para $J^{\circledast} E$, dado pela equação (1.67), e comparando esta com as equações (1.136) e (1.137), chegamos à

Proposição 1.7. Seja $X \in \mathfrak{X}\left(J^{\circledast} E\right)$ um campo vetorial qualquer sobre o espaço de multifase estendido $J^{\oplus E}$. Então $X$ é exatamente hamiltoniano se e somente se $X$ é projetável sobre $E$ e coincide com o levantamento canônico $X_{J^{\oplus} E}$ de sua projeção $X_{E}$.

Passando a campos vetoriais localmente mas não necessariamente exatamente hamiltonianos, o terceiro item do Teorema (1.2) sugere uma decomposição de $X$ na soma de dois termos, sendo o primeiro o mesmo que no teorema anterior. A novidade reside no segundo termo, isto é, nos coeficientes $X_{-}^{\mu}$, cuja interpretação global decorre da seguinte proposição:

Proposição 1.8 Seja $f_{0}$ uma $(n-1)$-forma sobre E e considere a $(n-1)$-forma sobre $J^{\otimes} E$ obtida de $f_{0}$ por pull-back pela projeção alvo $\tau^{\oplus}: J^{\circledast} E \rightarrow E$, que por abuso de notação também será denotada por $f_{0}$. Então as seguintes afirmações são equivalentes:

- Sobre $J^{\circledast} E$, $f_{0}$ é hamiltoniana.

- Sobre E, a derivada exterior $d f_{0}$ de $f_{0}$ é $(n-1)$-horizontal.

Em coordenadas locais adaptadas, podemos escrever

$$
f_{0}=f_{0}^{\mu} d^{n} x_{\mu}+\frac{1}{2}\left(f_{0}\right)_{i}^{\mu \nu} d q^{i} \wedge d^{n} x_{\mu \nu}+\ldots,
$$


onde os pontos denotam termos de ordem superior contendo pelo menos dois dq's, $e$

$$
d f_{0}=\frac{\partial f_{0}^{\mu}}{\partial x^{\mu}} d^{n} x+\left(\frac{\partial f_{0}^{\mu}}{\partial q^{i}}-\frac{\partial\left(f_{0}\right)_{i}^{\mu \nu}}{\partial x^{\nu}}\right) d q^{i} \wedge d^{n} x_{\mu},
$$

obtendo

$$
X_{f_{0}}=-\left(\frac{\partial f_{0}^{\mu}}{\partial q^{i}}-\frac{\partial\left(f_{0}\right)_{i}^{\mu \nu}}{\partial x^{\nu}}\right) \frac{\partial}{\partial p_{i}^{\mu}}-\frac{\partial f_{0}^{\mu}}{\partial x^{\mu}} \frac{\partial}{\partial p} .
$$

para o campo hamiltoniano associado sobre $J^{\circledast} E$.

DEMONSTRAÇÃO. A representação de uma $(n-1)$-forma geral sobre $E$ em coordenadas locais adaptadas sendo dada pela eq. (1.146), podemos aplicar a derivada exterior para obter uma fórmula que difere da eq. (1.147) pela presença de termos de ordem superior contendo pelo menos dois $d q$ 's. A ausência destes termos expressa, de um lado, a $(n-1)$-horizontalidade de $d f_{0}$ sobre $E$ e, de outro lado, a propriedade de $d f_{0}$ sobre $J^{\circledast} E$ se anular sobre o núcleo de $\omega$, pois tendo em vista a forma explícita (1.82) de $\omega$ em coordenadas locais adaptadas, mostra-se que um multivetor que é uma combinação linear de produtos exteriores de vetores da forma $\partial_{\mu}$ e $\partial_{i}$ pertence ao núcleo de $\omega$ se e somente se cada dos seus termos contém pelo menos dois vetores do tipo $\partial_{i}$. Da mesma maneira, a forma explícita (1.82) de $\omega$ em coordenadas locais adaptadas mostra que se definirmos $X_{f_{0}}$ pela fórmula (1.148), a condição (1.124) será satisfeita.

Agora podemos formular a decomposição global de campos vetoriais localmente hamiltonianos em componentes homogêneas sob $L_{\Sigma}$ :

Teorema 1.3 Seja $X \in \mathfrak{X}\left(J^{\circledast} E\right)$ um campo vetorial localmente hamiltoniano sobre o espaço de multifase estendido $J^{\oplus E}$. Então $X$ admite uma decomposição canônica na soma de dois termos

$$
X=X_{-}+X_{0}
$$

onde $X_{-}$e $X_{0}$ são campos vetoriais localmente hamiltonianos homogêneos de grau -1 e 0 sob $L_{\Sigma}$, respectivamente. Ademais, temos que

- $X_{-}$é vertical em relação à projeção sobre E e obtido por pull-back e dualizaçáo sob $\omega$ conforme exposto na Proposição 1.8.

- $X_{0}$ é exatamente hamiltoniano e obtido por levantamento canônico conforme exposto na Proposição 1.7. 


\subsubsection{Espaço de multifase comum}

No caso do espaço de multifase comum $\vec{J}^{\circledast} E$, prosseguimos de forma análoga, por cálculo direto em coordenadas locais adaptadas, em termos das quais um campo vetorial $\vec{X}$ qualquer sobre $\vec{J}^{\otimes} E$ assume a forma

$$
\vec{X}=X^{\mu} \frac{\partial}{\partial x^{\mu}}+X^{i} \frac{\partial}{\partial q^{i}}+X_{i}^{\mu} \frac{\partial}{\partial p_{i}^{\mu}},
$$

onde todos os coeficientes $X^{\mu}, X^{i}$ e $X_{i}^{\mu}$ são funções (em geral arbitrárias) das variáveis $x^{\kappa}, q^{k}$ e $p_{k}^{\kappa}$. Tendo em vista as formas explícitas (1.87) de $\omega_{\mathcal{H}}$ e (1.85) de $\theta_{\mathcal{H}}$ em coordenadas locais adaptadas, torna-se óbvio que

$$
\begin{aligned}
i_{X^{\prime}} \omega_{\mathcal{H}}= & X^{\nu} d q^{i} \wedge d p_{i}^{\mu} \wedge d^{n} x_{\mu \nu}-\left(X_{i}^{\mu}+X^{\mu} \frac{\partial H}{\partial q^{i}}\right) d q^{i} \wedge d^{n} x_{\mu} \\
& +\left(X^{i} \delta_{\mu}^{\nu}-X^{\nu} \frac{\partial H}{\partial p_{i}^{\mu}}\right) d p_{i}^{\mu} \wedge d^{n} x_{\nu}+\left(X^{i} \frac{\partial H}{\partial q^{i}}+X_{i}^{\mu} \frac{\partial H}{\partial p_{i}^{\mu}}\right) d^{n} x .
\end{aligned}
$$

$\mathrm{e}$

$$
i_{\vec{X}} \theta_{\mathcal{H}}=\left(p_{i}^{\mu} X^{i}-H X^{\mu}\right) d^{n} x_{\mu}-p_{i}^{\mu} X^{\nu} d q^{i} \wedge d^{n} x_{\mu \nu}
$$

Estas fórmulas constituem o ponto de partida para a demonstração do seguinte teorema.

Teorema 1.4 Seja $\vec{X} \in \mathfrak{X}\left(\vec{J}^{\otimes} E\right)$ um campo vetorial exatamente hamiltoniano sobre o espaço de multifase comum $\overrightarrow{J^{\circledast}} E$. Então em coordenadas locais adaptadas, as componentes de $\vec{X}$ conforme definidas pela equação (1.150) acima estão sujeitas às seguintes restrições:

- Os coeficientes $X^{\mu}$ independem das variáveis $p_{k}^{\kappa}$. Para $N>1$, também independem das variáveis $q^{k}$.

- Os coeficientes $X^{i}$ independem das variáveis $p_{k}^{\kappa}$.

- Os coeficientes $X_{i}^{\mu}$ são definidos em termos dos coeficientes $X^{\mu}$ e $X^{i}$ pela seguinte fórmula:

$$
X_{i}^{\mu}=H \frac{\partial X^{\mu}}{\partial q^{i}}-p_{j}^{\mu} \frac{\partial X^{j}}{\partial q^{i}}+p_{i}^{\nu} \frac{\partial X^{\mu}}{\partial x^{\nu}}-p_{i}^{\mu} \frac{\partial X^{\nu}}{\partial x^{\nu}}
$$

- Vale a seguinte relação de compatibilidade entre os coeficientes $X^{\mu}$ e $X^{i}$ e a função hamiltoniana $H$ :

$$
\text { - } H \frac{\partial X^{\mu}}{\partial x^{\mu}}+p_{i}^{\mu} \frac{\partial X^{i}}{\partial x^{\mu}}=\frac{\partial H}{\partial x^{\mu}} X^{\mu}+\frac{\partial H}{\partial q^{i}} X^{i}+\frac{\partial H}{\partial p_{i}^{\mu}} X_{i}^{\mu} .
$$


DemonstraÇÃo. Seja $\vec{X} \in \mathfrak{X}\left(\vec{J}^{\circledast} E\right)$ um campo vetorial qualquer sobre $\vec{J}^{\circledast} E$. Em coordenadas locais adaptadas, aplicamos a derivada exterior $d$ à equação (1.152) para obter

$$
\begin{aligned}
d\left(i_{\vec{X}} \theta_{\mathcal{H}}\right)= & X^{i} d p_{i}^{\mu} \wedge d^{n} x_{\mu}+\frac{\partial X^{i}}{\partial x^{\mu}} p_{i}^{\mu} d^{n} x \\
& +\frac{\partial X^{i}}{\partial q^{j}} p_{i}^{\mu} d q^{j} \wedge d^{n} x_{\mu}+\frac{\partial X^{i}}{\partial p_{j}^{\nu}} p_{i}^{\mu} d p_{j}^{\nu} \wedge d^{n} x_{\mu} \\
& -X^{\mu} \frac{\partial H}{\partial x^{\mu}} d^{n} x-X^{\mu} \frac{\partial H}{\partial q^{i}} d q^{i} \wedge d^{n} x_{\mu}-X^{\nu} \frac{\partial H}{\partial p_{i}^{\mu}} d p_{i}^{\mu} \wedge d^{n} x_{\nu} \\
& -\frac{\partial X^{\mu}}{\partial x^{\mu}} H d^{n} x-\frac{\partial X^{\mu}}{\partial q^{i}} H d q^{i} \wedge d^{n} x_{\mu}-\frac{\partial X^{\mu}}{\partial p_{j}^{\nu}} H d p_{j}^{\nu} \wedge d^{n} x_{\mu} \\
& -X^{\nu} d p_{i}^{\mu} \wedge d q^{i} \wedge d^{n} x_{\mu \nu}+\frac{\partial X^{\nu}}{\partial x^{\kappa}} p_{i}^{\mu} d q^{i} \wedge d x^{\kappa} \wedge d^{n} x_{\mu \nu} \\
& -\frac{\partial X^{\nu}}{\partial q^{j}} p_{i}^{\mu} d q^{j} \wedge d q^{i} \wedge d^{n} x_{\mu \nu}-\frac{\partial X^{\nu}}{\partial p_{k}^{\kappa}} p_{i}^{\mu} d p_{k}^{\kappa} \wedge d q^{i} \wedge d^{n} x_{\mu \nu} .
\end{aligned}
$$

Assim, pela equação (1.151),

$$
\begin{aligned}
L_{\vec{X}} \theta_{\mathcal{H}}= & d\left(i_{\vec{X}} \theta_{\mathcal{H}}\right)-i_{\vec{X}} \omega_{\mathcal{H}} \\
= & \left(-\frac{\partial X^{\mu}}{\partial x^{\mu}} H+\frac{\partial X^{i}}{\partial x^{\mu}} p_{i}^{\mu}-\left(\frac{\partial H}{\partial x^{\mu}} X^{\mu}+\frac{\partial H}{\partial q^{i}} X^{i}+\frac{\partial H}{\partial p_{i}^{\mu}} X_{i}^{\mu}\right)\right) d^{n} x \\
& +\left(\frac{\partial X^{j}}{\partial q^{i}} p_{j}^{\mu}-\frac{\partial X^{\mu}}{\partial x^{\nu}} p_{i}^{\nu}+\frac{\partial X^{\nu}}{\partial x^{\nu}} p_{i}^{\mu}-\frac{\partial X^{\mu}}{\partial q^{i}} H+X_{i}^{\mu}\right) d q^{i} \wedge d^{n} x_{\mu} \\
& +\left(\frac{\partial X^{i}}{\partial p_{j}^{\nu}} p_{i}^{\mu}-\frac{\partial X^{\mu}}{\partial p_{j}^{\nu}} H\right) d p_{j}^{\nu} \wedge d^{n} x_{\mu} \\
& +\frac{\partial X^{\nu}}{\partial q^{j}} p_{i}^{\mu} d q^{i} \wedge d q^{j} \wedge d^{n} x_{\mu \nu} \\
& +\frac{\partial X^{\nu}}{\partial p_{k}^{\kappa}} p_{i}^{\mu} d q^{i} \wedge d p_{k}^{\kappa} \wedge d^{n} x_{\mu \nu} .
\end{aligned}
$$

Numerando os termos nesta equação de 1 a 5 , a condição

$$
L_{\vec{X}} \theta_{\mathcal{H}}=0
$$

leva às seguintes conclusões: 
- Termo 5: Este se anula se e somente se os coeficientes $X^{\mu}$ independem das variáveis $p_{k}^{\kappa}$, e então o terceiro termo se anula se e somente se os coeficientes $X^{i}$ independem das variáveis $p_{k}^{\kappa}$.

- Termo 4: Este se anula se e somente se os coeficientes $X^{\mu}$ independem das variáveis $q^{k}$, caso $N>1$. No caso $N=1$, este termo se anula identicamente.

- Termo 2: Este se anula se e somente se os coeficientes $X_{i}^{\mu}$ são definidos em termos dos coeficientes $X^{\mu}$ e $X^{i}$ segundo a equação (1.153).

- Termo 1: Este se anula se e somente se vale a relação (1.154).

Passando a campos vetoriais localmente mas não necessariamente exatamente hamiltonianos, temos o seguinte teorema.

Teorema 1.5 Seja $\vec{X} \in \mathfrak{X}\left(\vec{J}^{\circledast} E\right)$ um campo vetorial localmente hamiltoniano sobre o espaço de multifase comum $\vec{J}^{\otimes} E$, e suponha que $N>1$ ou $n>2$. Então em coordenadas locais adaptadas, as componentes de $\vec{X}$ conforme definidas pela equação (1.150) acima estão sujeitas às seguintes restrições:

- Os coeficientes $X^{\mu}$ independem das variáveis $p_{k}^{\kappa}$. Para $N>1$, também independem das variáveis $q^{k}$.

- Os coeficientes $X^{i}$ independem das variáveis $p_{k}^{\kappa}$.

- Os coeficientes $X_{i}^{\mu}$ são definidos em termos dos coeficientes $X^{\mu}$ e $X^{i}$, em conjunto com novos coeficientes $X_{-}^{\mu}$ que também independem das variáveis $p_{k}^{\kappa}$, pela seguinte fórmula:

$$
X_{i}^{\mu}=H \frac{\partial X^{\mu}}{\partial q^{i}}-p_{j}^{\mu} \frac{\partial X^{j}}{\partial q^{i}}+p_{i}^{\nu} \frac{\partial X^{\mu}}{\partial x^{\nu}}-p_{i}^{\mu} \frac{\partial X^{\nu}}{\partial x^{\nu}}+\frac{\partial X_{-}^{\mu}}{\partial q^{i}} .
$$

- Vale a seguinte relação de compatibilidade entre os coeficientes $X^{\mu}, X^{i}$ e $X_{-}^{\mu}$ e a função hamiltoniana $H$ :

$$
-H \frac{\partial X^{\mu}}{\partial x^{\mu}}+p_{i}^{\mu} \frac{\partial X^{i}}{\partial x^{\mu}}-\frac{\partial X_{-}^{\mu}}{\partial x^{\mu}}=\frac{\partial H}{\partial x^{\mu}} X^{\mu}+\frac{\partial H}{\partial q^{i}} X^{i}+\frac{\partial H}{\partial p_{i}^{\mu}} X_{i}^{\mu} .
$$

Observação 1.1 Em relação às afirmações do Teorema 1.5, o caso em que $N=1$ e $n=2$ parece ser excepcional. Por exemplo, os argumentos a serem apresentados na demonstração 
a seguir não permitem concluir, neste caso, que os coeficientes $X^{\mu}$ e $X^{i}$ independem das variáveis $p_{k}^{\kappa}$; mas apenas que vale ${ }^{6}$

e

$$
\begin{gathered}
\frac{\partial X^{\mu}}{\partial p^{\mu}}=0 \\
\frac{\partial X}{\partial p^{\mu}}=\frac{\partial X^{\nu}}{\partial p^{\mu}} \frac{\partial H}{\partial p^{\nu}}
\end{gathered}
$$

DemonstraÇÃo. Seja $\vec{X} \in \mathfrak{X}\left(\vec{J}{ }^{\circledast} E\right)$ um campo vetorial qualquer sobre $\vec{J}^{\circledast} E$. Em coordenadas locais adaptadas, aplicamos a derivada exterior $d$ à equação (1.151) para obter

$$
\begin{aligned}
L_{\vec{X}} \omega_{\mathcal{H}}= & d\left(i_{X^{\prime}} \omega_{\mathcal{H}}\right) \\
= & \frac{\partial X^{\nu}}{\partial x^{\kappa}} d q^{i} \wedge d p_{i}^{\mu} \wedge d x^{\kappa} \wedge d^{n} x_{\mu \nu}+\frac{\partial X^{\nu}}{\partial q^{j}} d q^{j} \wedge d q^{i} \wedge d p_{i}^{\mu} \wedge d^{n} x_{\mu \nu} \\
& +\frac{\partial X^{\nu}}{\partial p_{k}^{\kappa}} d p_{k}^{\kappa} \wedge d q^{i} \wedge d p_{i}^{\mu} \wedge d^{n} x_{\mu \nu} \\
& +\frac{\partial}{\partial x^{\mu}}\left(X_{i}^{\mu}+X^{\mu} \frac{\partial H}{\partial q^{i}}\right) d q^{i} \wedge d^{n} x \\
& -\frac{\partial}{\partial q^{j}}\left(X_{i}^{\mu}+X^{\mu} \frac{\partial H}{\partial q^{i}}\right) d q^{j} \wedge d q^{i} \wedge d^{n} x_{\mu} \\
& -\frac{\partial}{\partial p_{j}^{\nu}}\left(X_{i}^{\mu}+X^{\mu} \frac{\partial H}{\partial q^{i}}\right) d p_{j}^{\nu} \wedge d q^{i} \wedge d^{n} x_{\mu} \\
& -\frac{\partial}{\partial x^{\nu}}\left(\delta_{\mu}^{\nu} X^{i}-X^{\nu} \frac{\partial H}{\partial p_{i}^{\mu}}\right) d p_{i}^{\mu} \wedge d^{n} x \\
& +\frac{\partial}{\partial q^{j}}\left(\delta_{\mu}^{\nu} X^{i}-X^{\nu} \frac{\partial H}{\partial p_{i}^{\mu}}\right) d q^{j} \wedge d p_{i}^{\mu} \wedge d^{n} x_{\nu} \\
& +\frac{\partial}{\partial p_{j}^{\nu}}\left(\delta_{\mu}^{\nu} X^{i}-X^{\kappa} \frac{\partial H}{\partial p_{i}^{\mu}}\right) d p_{j}^{\nu} \wedge d p_{i}^{\mu} \wedge d^{n} x_{\kappa} \\
& +\frac{\partial}{\partial q^{i}}\left(X^{j} \frac{\partial H}{\partial q^{j}}+X_{j}^{\nu} \frac{\partial H}{\partial p_{j}^{\nu}}\right) d q^{i} \wedge d^{n} x \\
& +\frac{\partial}{\partial p_{i}^{\mu}}\left(X^{j} \frac{\partial H}{\partial q^{j}}+X_{j}^{\nu} \frac{\partial H}{\partial p_{j}^{\nu}}\right) d p_{i}^{\mu} \wedge d^{n} x \\
& \\
& \\
& \\
&
\end{aligned}
$$

\footnotetext{
${ }^{6}$ Lembramos que quando $N=1$, omitimos os índices $i, j$, etc.
} 
Reagrupando os termos, obtemos

$$
\begin{aligned}
L_{\mathbb{X}^{*}} \omega_{\mathcal{H}}= & \left(\frac{\partial}{\partial x^{\mu}}\left(X_{i}^{\mu}+X^{\mu} \frac{\partial H}{\partial q^{i}}\right)+\frac{\partial}{\partial q^{i}}\left(X^{j} \frac{\partial H}{\partial q^{j}}+X_{j}^{\nu} \frac{\partial H}{\partial p_{j}^{\nu}}\right)\right) d q^{i} \wedge d^{n} x \\
& -\left(\frac{\partial}{\partial x^{\nu}}\left(\delta_{\mu}^{\nu} X^{i}-X^{\nu} \frac{\partial H}{\partial p_{i}^{\mu}}\right)-\frac{\partial}{\partial p_{i}^{\mu}}\left(X^{j} \frac{\partial H}{\partial q^{j}}+X_{j}^{\nu} \frac{\partial H}{\partial p_{j}^{\nu}}\right)\right) d p_{i}^{\mu} \wedge d^{n} x \\
& +\left(\delta_{l}^{k}\left(\delta_{\kappa}^{\sigma} \frac{\partial X^{\tau}}{\partial x^{\tau}}-\frac{\partial X^{\sigma}}{\partial x^{\kappa}}\right)\right. \\
& \left.+\frac{\partial}{\partial p_{k}^{\kappa}}\left(X_{l}^{\sigma}+X^{\sigma} \frac{\partial H}{\partial q^{l}}\right)+\frac{\partial}{\partial q^{l}}\left(\delta_{\kappa}^{\sigma} X^{k}-X^{\sigma} \frac{\partial H}{\partial p_{k}^{\kappa}}\right)\right) d q^{l} \wedge d p_{k}^{\kappa} \wedge d^{n} x_{\sigma} \\
& -\frac{\partial}{\partial q^{j}}\left(X_{i}^{\mu}+X^{\mu} \frac{\partial H}{\partial q^{i}}\right) d q^{j} \wedge d q^{i} \wedge d^{n} x_{\mu} \\
& +\frac{\partial}{\partial p_{k}^{\kappa}}\left(\delta_{\lambda}^{\sigma} X^{l}-X^{\sigma} \frac{\partial H}{\partial p_{l}^{\lambda}}\right) d p_{k}^{\kappa} \wedge d p_{l}^{\lambda} \wedge d^{n} x_{\sigma} \\
& +\frac{\partial X^{\sigma}}{\partial q^{k}} d q^{k} \wedge d q^{l} \wedge d p_{l}^{\lambda} \wedge d^{n} x_{\lambda \sigma} \\
& -\frac{\partial X^{\sigma}}{\partial p_{k}^{\kappa}} d q^{l} \wedge d p_{k}^{\kappa} \wedge d p_{l}^{\lambda} \wedge d^{n} x_{\lambda \sigma} .
\end{aligned}
$$

Numerando os termos nesta equação de 1 a 7, a condição

$$
L_{\vec{X}} \omega_{\mathcal{H}}=0
$$

leva às seguintes conclusões:

- Termo 7: Pelo mesmo argumento usado na demonstração do Teorema 1.2 para analisar o termo 10 , podemos concluir que

$$
\frac{\partial X^{\rho}}{\partial p_{i}^{\mu}}=0
$$

exceto no caso em que $N=1$ e $n=2$, onde é apenas possível concluir que vale a relação (1.157).

- Termo 5: Fixando índices $i, j, \mu, \nu, \rho$ e $\rho_{1}, \ldots, \rho_{n-1}$ tais que os índices $\rho, \rho_{1}, \ldots, \rho_{n-1}$ são mutuamente diferentes, contraímos $L_{\vec{X}} \omega_{\mathcal{H}}$ com o campo multivetorial 
$\partial_{\mu}^{i} \wedge \partial_{\nu}^{j} \wedge \partial_{\rho_{1}} \wedge \ldots \wedge \partial_{\rho_{n-1}}$, obtendo a relação

$$
\frac{\partial}{\partial p_{i}^{\mu}}\left(\delta_{\nu}^{\rho} X^{j}-X^{\rho} \frac{\partial H}{\partial p_{j}^{\nu}}\right)=\frac{\partial}{\partial p_{j}^{\nu}}\left(\delta_{\mu}^{\rho} X^{i}-X^{\rho} \frac{\partial H}{\partial p_{i}^{\mu}}\right),
$$

ou seja.

$$
\delta_{\nu}^{\rho} \frac{\partial X^{j}}{\partial p_{i}^{\mu}}-\frac{\partial X^{\rho}}{\partial p_{i}^{\mu}} \frac{\partial H}{\partial p_{j}^{\nu}}=\delta_{\mu}^{\rho} \frac{\partial X^{i}}{\partial p_{j}^{\nu}}-\frac{\partial X^{\rho}}{\partial p_{j}^{\nu}} \frac{\partial H}{\partial p_{i}^{\mu}} .
$$

Quando $N>1$ ou $n>2$, podemos usar o resultado do item anterior para concluir que

$$
\delta_{\nu}^{\rho} \frac{\partial X^{j}}{\partial p_{i}^{\mu}}=\delta_{\mu}^{\rho} \frac{\partial X^{i}}{\partial p_{j}^{\nu}}
$$

Agora, se inicialmente, fixamos apenas os índices $i, j$ e $\mu$, podemos sempre escolher os demais índices tais que $\nu \neq \mu$ e $\rho=\nu$, o que reduz o lado esquerdo desta equação à expressão $\partial X^{j} / \partial p_{i}^{\mu}$, enquanto que o lado direito se anula. Assim, chegamos à conclusão de que

$$
\frac{\partial X^{j}}{\partial p_{i}^{\mu}}=0
$$

Por outro lado, quando $N=1$ e $n=2$, a equação anterior assume a forma

$$
\delta_{\nu}^{\rho} \frac{\partial X}{\partial p^{\mu}}-\frac{\partial X^{\rho}}{\partial p^{\mu}} \frac{\partial H}{\partial p^{\nu}}=\delta_{\mu}^{\rho} \frac{\partial X}{\partial p^{\nu}}-\frac{\partial X^{\rho}}{\partial p^{\nu}} \frac{\partial H}{\partial p^{\mu}} .
$$

Agora, se inicialmente, fixamos apenas o índice $\mu$, podemos sempre escolher os demais índices tais que $\nu \neq \mu$ e $\rho=\nu$, obtendo

$$
\frac{\partial X}{\partial p^{\mu}}=\frac{\partial X^{\nu}}{\partial p^{\mu}} \frac{\partial H}{\partial p^{\nu}}-\frac{\partial X^{\nu}}{\partial p^{\nu}} \frac{\partial H}{\partial p^{\mu}} \quad(\text { sem soma sobre } \nu)
$$

o que, devido à equação (1.157), é equivalente à equação (1.158).

- Termo 6: Pelo mesmo argumento usado na demonstração do Teorema 1.2 para analisar o termo 9, podemos concluir que

$$
\frac{\partial X^{p}}{\partial q^{i}}=0
$$

exceto no caso em que $N=1$ onde o termo inteiro se anula identicamente e nenhuma conclusão pode ser tirada.

Para prosseguir, escrevemos as equações obtidas dos termos restantes. 
- Termo 1:

$$
\frac{\partial}{\partial q^{i}}\left(X^{j} \frac{\partial H}{\partial q^{j}}+X_{j}^{\nu} \frac{\partial H}{\partial p_{j}^{\nu}}\right)=-\frac{\partial}{\partial x^{\mu}}\left(X_{i}^{\mu}+X^{\mu} \frac{\partial H}{\partial q^{i}}\right) .
$$

- Termo 2:

$$
\frac{\partial}{\partial p_{i}^{\mu}}\left(X^{j} \frac{\partial H}{\partial q^{j}}+X_{j}^{\nu} \frac{\partial H}{\partial p_{j}^{\nu}}\right)=\frac{\partial}{\partial x^{\nu}}\left(\delta_{\mu}^{\nu} X^{i}-X^{\nu} \frac{\partial H}{\partial p_{i}^{\mu}}\right) .
$$

- Termo 3:

$$
\begin{aligned}
\frac{\partial}{\partial p_{j}^{\nu}}\left(X_{i}^{\mu}+X^{\mu} \frac{\partial H}{\partial q^{i}}\right)= & -\frac{\partial}{\partial q^{i}}\left(\delta_{\nu}^{\mu} X^{j}-X^{\mu} \frac{\partial H}{\partial p_{j}^{\nu}}\right) \\
& +\delta_{i}^{j} \frac{\partial X^{\mu}}{\partial x^{\nu}}-\delta_{\nu}^{\mu} \delta_{i}^{j} \frac{\partial X^{\kappa}}{\partial x^{\kappa}}
\end{aligned}
$$

- Termo 4:

$$
\frac{\partial X_{i}^{\mu}}{\partial q^{j}}=\frac{\partial X_{j}^{\mu}}{\partial q^{i}}
$$

Supondo a partir de agora que $N>1$ ou $n>2$ e usando que sob esta hipótese, os coeficientes $X^{\mu}$ e $X^{i}$ independem das variáveis $p_{k}^{\kappa}$, notamos que a equação (1.161) pode ser integrada diretamente para concluir que

$$
X_{i}^{\mu}=H \frac{\partial X^{\mu}}{\partial q^{i}}-p_{j}^{\mu} \frac{\partial X^{j}}{\partial q^{i}}+p_{i}^{\nu} \frac{\partial X^{\mu}}{\partial x^{\nu}}-p_{i}^{\mu} \frac{\partial X^{\kappa}}{\partial x^{\kappa}}+Y_{i}^{\mu}
$$

onde os $Y_{i}^{\mu}$ são funções locais sobre $E$ : independem das variáveis $p_{k}^{\kappa}$. Substituindo esta relação na equação (1.162), vem

o que pode ser resolvido pondo

$$
\frac{\partial Y_{i}^{\mu}}{\partial q^{j}}=\frac{\partial Y_{j}^{\mu}}{\partial q^{i}}
$$

$$
Y_{i}^{\mu}=\frac{\partial Y_{-}^{\mu}}{\partial q^{i}}
$$

onde os $Y_{-}^{\mu}$ são funções locais sobre $E$ : independem das variáveis $p_{k}^{\kappa}$. Finalmente, substituindo a relação assim obtida nas equações (1.159) e (1.160), vem

$$
\frac{\partial}{\partial q^{i}}\left(H \frac{\partial X^{\nu}}{\partial x^{\nu}}-p_{j}^{\nu} \frac{\partial X^{j}}{\partial x^{\nu}}+\frac{\partial Y_{-}^{\nu}}{\partial x^{\nu}}+\frac{\partial H}{\partial x^{\nu}} X^{\nu}+\frac{\partial H}{\partial q^{j}} X^{j}+\frac{\partial H}{\partial p_{j}^{\nu}} X_{j}^{\nu}\right)=0
$$

e

$$
\frac{\partial}{\partial p_{i}^{\mu}}\left(H \frac{\partial X^{\nu}}{\partial x^{\nu}}-p_{j}^{\nu} \frac{\partial X^{j}}{\partial x^{\nu}}+\frac{\partial Y_{-}^{\nu}}{\partial x^{\nu}}+\frac{\partial H}{\partial x^{\nu}} X^{\nu}+\frac{\partial H}{\partial q^{j}} X^{j}+\frac{\partial H}{\partial p_{j}^{\nu}} X_{j}^{\nu}\right)=0
$$


mostrando que

$$
H \frac{\partial X^{\nu}}{\partial x^{\nu}}-p_{j}^{\nu} \frac{\partial X^{j}}{\partial x^{\nu}}+\frac{\partial Y_{-}^{\nu}}{\partial x^{\nu}}+\frac{\partial H}{\partial x^{\nu}} X^{\nu}+\frac{\partial H}{\partial q^{j}} X^{j}+\frac{\partial H}{\partial p_{j}^{\nu}} X_{j}^{\nu}=Y_{-}
$$

onde $Y_{-}$é uma função local sobre $M$ : independe das variáveis $q^{k}$ e $p_{k}^{\kappa}$. Escrevendo $Y_{-}$como uma divergência,

$$
Y_{-}=\frac{\partial Y_{-}^{\nu}}{\partial x^{\nu}}
$$

e pondo $X^{\mu}=Y_{-}^{\nu}-Y_{-}^{\prime \nu}$, chegamos às equações (1.155) e (1.156), o que conclui a demonstração do teorema.

Para encontrar uma interpretação global das conclusões destes teoremas, observamos novamente que os primeiros dois itens afirmam que um campo vetorial $\vec{X}$ (exatamente ou localmente) hamiltoniano sobre $\vec{J}^{\circledast} E$ é projetável sobre um campo vetorial $X_{E}$ sobre $E^{7}$ que, para $N>1$, por sua vez é projetável sobre um campo vetorial $X_{M}$ sobre $M$. Usando o levantamento canônico $X_{J^{\oplus} E}$ de $X_{E}$ para o espaço de multifase estendido $J^{\circledR} E$, dado pela equação (1.67), e comparando esta com as equações (1.153) e (1.154), chegamos à

Proposição 1.9 Seja $\vec{X} \in \mathfrak{X}\left(\vec{J}^{\circledast} E\right)$ um campo vetorial qualquer sobre o espaço de multifase comum $\vec{J}{ }^{\otimes} E$. Então $\vec{X}$ é exatamente hamiltoniano se e somente se $\vec{X}$ é projetável sobre $E$ e é $\mathcal{H}$-relacionado ao levantamento canônico $X_{J{ }^{\oplus E}}$ de sua projeção $X_{E}$ :

$$
X_{J^{\circledast} E} \circ \mathcal{H}=T \mathcal{H} \circ \vec{X} .
$$

Passando a campos vetoriais localmente mas não necessariamente exatamente hamiltonianos, o terceiro item do Teorema (1.5) sugere uma decomposição de $\vec{X}$ na soma de dois termos, como antes. No entanto, a interpretação global desta decomposição parece ser mais complicada do que no caso do Teorema 1.2. A razão principal da dificuldade reside no fato de que, apesar de ainda ser possível caracterizar esta decomposição em termos de componentes homogêneos sob a derivada de Lie ao longo do campo de Euler $\Sigma$ (agora sobre $\vec{J}{ }^{\circledR} E$ ), estes dois componentes deixam de ser localmente hamiltonianos pois não satisfazem separadamente o vínculo (1.156). Assim, o Teorema 1.3 parece não ter nenhum análogo natural.

\footnotetext{
${ }^{7}$ Lembramos que excluímos o caso excepcional de campos vetoriais localmente hamiltonianos mencionado na Observação 1.1 (sistemas com $N=1$ grau de liberdade em $n=2$ dimensões), que requer um tratamento separado.
} 


\subsubsection{Projeção e levantamento de campos vetoriais hamiltonianos}

O passo final no nosso estudo de campos vetoriais hamiltonianos consistirá em esclarecer a relação entre campos vetoriais (exatamente ou localmente) hamiltonianos $X$ sobre o espaço de multifase estendido $J^{\otimes} E$ e campos vetoriais (exatamente ou localmente) hamiltonianos $\vec{X}$ sobre o espaço de multifase comum $\vec{J}^{\circledast} E$, no caso em que estes são projetáveis.

Definição $1.7 U m$ campo vetorial $X$ sobre o espaço de multifase estendido $J^{\circledR} E$ é chamado projetável se for projetável em relação a cada uma das três projeções (sobre $\vec{J}{ }^{\circledast} E$, sobre $E$ e sobre $M$ ). De forma análoga, um campo vetorial $\vec{X}$ sobre o espaço de multifase comum $\vec{J}{ }^{\circledast} E$ é chamado projetável se for projetável em relação a cada uma das duas projeções (sobre $E$ e sobre $M$ ).

Com esta terminologia, os Teoremas 1.2 e 1.5 implicam que para $N>1$, qualquer campo vetorial localmente hamiltoniano é projetável, enquanto que, talvez surpreendentemente, o caso de sistemas com apenas um grau de liberdade é mais complicado: Para $N=1$, um campo vetorial localmente hamiltoniano sobre $\vec{J}^{\otimes} E$ é projetável sobre $E$ se $n>2$, enquanto que um campo vetorial localmente hamiltoniano sobre $J^{\circledast} E$ é sempre projetável sobre $E$ e será projetável sobre $\vec{J}^{\otimes} E$ se e somente se for projetável sobre $M$.

Para a discussão a seguir, que aborda a relação entre campos vetoriais localmente hamiltonianos sobre $J^{\circledast} E$ e sobre $\vec{J}^{\circledast} E$, restringiremo-nos a campos vetoriais projetáveis, em todos os sentidos como definido acima, mesmo quando $N=1$. Um dos motivos é que campos vetoriais sobre o espaço total de um fibrado sobre o espaço-tempo $M$ que não sejam projetáveis sobre $M$ podem ser considerados como patológicos, pois geram um tipo de transformação que não induz nenhuma transformação bem-definida no espaço-tempo: é difícil imaginar como interpretar uma transformação desta natureza como candidato para uma simetria que tenha algum significado físico.

A relação entre campos vetoriais projetáveis e localmente hamiltonianos sobre $\vec{J}^{\otimes} E$ e sobre $J^{\circledast} E$ baseia-se na observação de que a relação de compatibilidade (1.156) identificada no Teorema 1.5 possui uma interpretação geométrica-natural, independente da escolha de coordenadas: Ela afirma que os campos vetoriais projetáveis $X$ sobre $J^{\circledast} E$ e $\vec{X}$ sobre $\vec{J}{ }^{\circledast} E$ dados, respectivamente, pelas equações $(1.133),(1.138),(1.139)$ e $(1.150),(1.155)$ são $\mathcal{H}$ relacionados:

$$
X \circ \mathcal{H}=T \mathcal{H} \circ \vec{X}
$$

Independentemente da questão se os campos vetoriais envolvidos são localmente hamiltonianos ou não, mostra-se útil introduzir uma terminologia específica para medir um possível desvio da validade desta relação. 
Definição 1.8 Dada uma hamiltoniana $\mathcal{H}$, seja $X$ um campo vetorial sobre o espaço de multifase estendido $J^{\circledast} E$ que é projetável, conforme a Definição 1.7, seja $\vec{X}$ sua projeção sobre o espaço de multifase comum $\vec{J}^{\circledast} E$ e seja $\tilde{X}$ o levantamento hamiltoniano de $\vec{X}$, conforme a Proposição 1.3. O campo vetorial

$$
X^{\mathcal{H}}=X-\tilde{X}
$$

sobre $J^{\circledast} E$ será chamado o desvio hamiltoniano de $X$.

Em coordenadas locais adaptadas, com componentes de $X$ e de $\vec{X}$ conforme definidas pela equações (1.133) e (1.150), temos

$$
X^{\mathcal{H}}=X_{0}^{\mathcal{H}} \frac{\partial}{\partial p}
$$

onde, conforme a equação (1.116),

$$
X_{0}^{\mathcal{H}}=X_{0}+(p+H) \frac{\partial X^{\mu}}{\partial x^{\mu}}+\frac{\partial H}{\partial x^{\mu}} X^{\mu}+\frac{\partial H}{\partial q^{i}} X^{i}+\frac{\partial H}{\partial p_{i}^{\mu}} X_{i}^{\mu} .
$$

Por definição, $X$ e $\vec{X}$ são $\mathcal{H}$-relacionados se e somente se $X^{\mathcal{H}}$ se anula sobre a imagem de $\mathcal{H}$ em $J^{\oplus} E$. Se $X$ for localmente hamiltoniano, então a equação (1.139) mostra que o coeficiente $X_{0}^{\mathcal{H}}$ independe de $p$ e vale

$$
X_{0}^{\mathcal{H}}=H \frac{\partial X^{\mu}}{\partial x^{\mu}}-p_{i}^{\mu} \frac{\partial X^{i}}{\partial x^{\mu}}+\frac{\partial X_{-}^{\mu}}{\partial x^{\mu}}+\frac{\partial H}{\partial x^{\mu}} X^{\mu}+\frac{\partial H}{\partial q^{i}} X^{i}+\frac{\partial H}{\partial p_{i}^{\mu}} X_{i}^{\mu} .
$$

Note também que o fato de que, por definição, $\tilde{X}$ e $\vec{X}$ são sempre $\mathcal{H}$-relacionados implica que, para qualquer forma diferencial $\alpha$ sobre $J^{\oplus} E$, vale a relação

$$
i_{\vec{X}}\left(\mathcal{H}^{*} \alpha\right)=\mathcal{H}^{*}\left(i_{\bar{X}} \alpha\right)
$$

ou seja,

$$
i_{\vec{X}}\left(\mathcal{H}^{*} \alpha\right)=\mathcal{H}^{*}\left(i_{X} \alpha\right)-\mathcal{H}^{*}\left(i_{X^{\mathcal{H}}} \alpha\right)
$$

Em particular, notamos que

$$
i_{X^{\mathcal{H}}} \theta=0, \quad i_{X^{\mathcal{H}}} \omega=-X_{0}^{\mathcal{H}} d^{n} x .
$$


Quanto à possibilidade de construir $\vec{X}$ a partir de $X$ e/ou vice versa, vale o seguinte Teorema 1.6 Dada uma hamiltoniana $\mathcal{H}$, seja $X \in \mathfrak{X}\left(J^{\oplus} E\right)$ um campo vetorial sobre o espaço de multifase estendido $J^{\otimes} E$ e $\vec{X} \in \mathfrak{X}\left(\vec{J}^{\otimes} E\right)$ um campo vetorial sobre o espaço de multifase comum $\vec{J}^{\otimes} E$, ambos projetáveis.

- Dado $X$, suponha que $\vec{X}$ seja sua projeção.

Se $X$ for localmente hamiltoniano (em relação a $\omega$ ) e for tangente à imagem de $\mathcal{H}$, então $X$ e $\vec{X}$ são $\mathcal{H}$-relacionados, $\vec{X}$ é localmente hamiltoniano (em relação a $\omega_{\mathcal{H}}$ ) $e$ $X$ é seu levantamento hamiltoniano.

- Dado $\vec{X}$, suponha que $X$ seja seu levantamento hamiltoniano.

Se $\vec{X}$ for localmente hamiltoniano (em relação a $\omega_{\mathcal{H}}$ ), então $X$ e $\vec{X}$ são $\mathcal{H}$-relacionados, $X$ é localmente hamiltoniano (em relação a $\omega$ ) e $\vec{X}$ é sua projeção.

Em particular, $\vec{X}$ é localmente hamiltoniano se e somente se $X$ é localmente hamiltoniano e tangente à imagem de $\mathcal{H}$, ou equivalentemente, $X^{\mathcal{H}}=0$.

DEMONSTRAÇÃo. Observe que $X$ é tangente à imagem de $\mathcal{H}$ se e somente se para todo $\vec{z} \in \vec{J} \otimes E$, existe $\vec{Y}_{\vec{z}} \in T_{\vec{z}}\left(\vec{J}^{\otimes} E\right)$ tal que $X(\mathcal{H}(\vec{z}))=T_{\vec{z}} \mathcal{H}\left(\vec{Y}_{\vec{z}}\right)$. Aplicando a tangente $T_{\mathcal{H}(\vec{z})} \eta$ à projeção $\eta$, concluímos que $\vec{Y}_{\vec{z}}=\vec{X}_{\vec{z}}$. As afirmações que se referem ao levantamento hamiltoniano seguem por comparação das equações (1.116), (1.133) com (1.139) e (1.156).

\subsection{Colchete de Poisson}

Um dos maiores problemas no formalismo multissimplético, ainda parcialmente em aberto, é a definição adequada do colchete de Poisson entre observáveis. Apesar de uma resposta definitiva talvez ainda não tenha sido encontrada, existem resultados parciais, que passamos a expor.

Uma das idéias que tem sido explorada na literatura é definir um colchete de Poisson usando como ponto de partida a mesma fórmula $i_{X} \omega=d f$ da mecânica, o que no entanto requer várias modificações - algumas delas óbvias, outras nem tanto. Por exemplo, o fato de que no formalismo multissimplético, a forma $\omega$ é de grau $n+1>2$ implica que os objetos $X$ passam a ser campos multivetoriais, ao invés de campos vetoriais, e os objetos $f$ passam a ser formas diferenciais, ao invés de funções. Como já foi visto, isso acarreta um certo grau de ambiguidade, pois em geral, $X$ é determinado por $f$ apenas a menos de um campo 
multivetorial tomando valores no núcleo de $\omega$ e, reciprocamente, $f$ é determinada por $X$ apenas a menos de uma forma fechada. No caso especial em que $X$ tem grau 1 e $f$ tem grau $n-1$, a primeira ambiguidade deixa de existir, mas a segunda permanece. Em outras palavras, temos o seguinte isomorfismo:

$$
\text { Campos vetoriais hamiltonianos } \longleftrightarrow \begin{gathered}
\text { Formas hamiltonianas } \\
\text { de grau } n-1 \\
\text { módulo formas fechadas }
\end{gathered}
$$

Uma primeira proposta para um colchete de Poisson entre duas $(n-1)$-formas hamiltonianas $f$ e $g$ é a mesma da mecânica, ou seja,

$$
\{f, g\}=i_{X_{g}} i_{X_{f}} \omega
$$

onde $X_{f}$ e $X_{g}$ são os campos hamiltonianos associados a $f$ e $g$. Infelizmente, esta proposta não satisfaz a identidade de Jacobi, pois para três $(n-1)$-formas hamiltonianas $f, g$ e $h$, temos

$$
\{f,\{g, h\}\}+\{g,\{h, f\}\}+\{h,\{f, g\}\}=d\left(i_{X_{f}} i_{X_{g}} i_{X_{h}} \omega\right) .
$$

Entretanto, no caso de variedades multissimpléticas exatas, este defeito pode ser corrigido adicionando um termo exato adequado:

Teorema 1.7 [11] Seja $P$ uma variedade multissimplética exata com $n$-forma multicanônica $\theta$ e $(n+1)$-forma multissimplética $\omega=-d \theta$. Para quaisquer duas $(n-1)$-formas hamiltonianas $f$ e $g$ sobre $P$, chamamos a $(n-1)$-forma hamiltoniana $\{f, g\}$ definida por

$$
\{f, g\}=i_{X_{g}} i_{X_{f}} \omega+d\left(i_{X_{g}} f-i_{X_{f}} g-i_{X_{g}} i_{X_{f}} \theta\right)
$$

onde $X_{f}$ e $X_{g}$ são os campos hamiltonianos associados a $f$ e $g$, respectivamente, o colchete de Poisson de $f$ e g. Este colchete satisfaz a identidade de Jacobi e prove o espaço das $(n-1)$-formas hamiltonianas sobre $P$ de uma estrutura de álgebra de Lie.

Para generalizar esta definição do colchete de Poisson a formas de qualquer grau, torna-se necessário estender algumas das operações do cálculo em variedades envolvendo campos vetoriais ao caso de campos multivetoriais. Primeiro, para um campo multivetorial decomponível $X=X_{1} \wedge \ldots \wedge X_{r}$, a contração de uma forma $\alpha \operatorname{com} X$ é definida como

$$
i_{X} \alpha=i_{X_{r}} \ldots i_{X_{1}} \alpha
$$

Segundo, o colchete de Lie entre campos vetoriais pode ser estendido para um colchete entre campos multivetoriais conhecido como o colchete de Schouten, sendo que o colchete 
de Schouten entre dois campos multivetoriais decomponíveis $X=X_{1} \wedge \ldots \wedge X_{r}$ de grau $r$ e $Y=Y_{1} \wedge \ldots \wedge Y_{s}$ de grau $s$ é o campo multivetorial de grau $r+s-1$ definido por

$$
\begin{aligned}
{\left[X_{1}\right.} & \left.\wedge \wedge X_{r}, Y_{1} \wedge \ldots \wedge Y_{s}\right] \\
=\sum_{i=1}^{r} \sum_{j=1}^{s}(-1)^{i+j}\left[X_{i}, Y_{j}\right] & \wedge X_{1} \wedge \ldots \wedge X_{i-1} \wedge X_{i+1} \wedge \ldots \wedge X_{r} \\
& \wedge Y_{1} \wedge \ldots \wedge Y_{i-1} \wedge Y_{i+1} \wedge \ldots \wedge Y_{s} .
\end{aligned}
$$

As propriedades principais do colchete de Schouten são, além de sua bilinearidade sobre $\mathbb{R}$, a antissimetria graduada,

$$
[X, Y]=-(-1)^{(r-1)(s-1)}[Y, X]
$$

a identidade de Jacobi graduada,

$$
\begin{aligned}
(-1)^{(r-1)(t-1)}[X,[Y, Z]] & +(-1)^{(s-1)(r-1)}[Y,[Z, X]] \\
& +(-1)^{(t-1)(s-1)}[Z,[X, Y]]=0
\end{aligned}
$$

e a regra de Leibniz graduada (em relação ao produto exterior),

$$
[Z, X \wedge Y]=[Z, X] \wedge Y+(-1)^{(r-1) s} X \wedge[Z, Y]
$$

Isso significa que o espaço dos campos multivetoriais $\mathfrak{X}^{\wedge}(M)$ sobre uma variedade $M$ qualquer é uma superálgebra de Poisson - o análogo supersimétrico de uma álgebra de Poisson. Finalmente, a derivada de Lie de uma forma diferencial ao longo de um campo multivetorial $X$ é definida como o comutador graduado da derivada exterior com a contração com $X$ :

$$
L_{X} \alpha=\left(d i_{X}-(-1)^{r} i_{X} d\right) \alpha .
$$

Retornando à questão da correspondência entre campos multivetoriais hamiltonianos de grau $r$ e formas hamiltonianas de grau $n-r$ estabelecida pela fórmula $i_{X} \omega=d f$, observamos que para $r$ geral, o isomorfismo (1.172) deve ser substituído pelo seguinte isomorfismo:

$$
\begin{gathered}
\text { Campos multivetoriais } \\
\begin{array}{c}
\text { hamiltonianos de grau } r \\
\text { módulo núcleo de } \omega
\end{array}
\end{gathered} \begin{gathered}
\begin{array}{c}
\text { Formas hamiltonianas } \\
\text { de grau } n-r
\end{array} \\
\text { módulo formas fechadas }
\end{gathered}
$$

Devido à falta de unicidade dos campos multivetoriais associados às formas hamiltonianas, teremos que impor uma restrição adicional sobre estas para garantir que o colchete de Poisson a ser introduzido abaixo seja bem definido.

Definição 1.9 [12] Seja P uma variedade multissimplética com forma multissimplética $\omega$. Uma forma hamiltoniana $f$ sobre $P$ é chamada uma forma de Poisson se o núcleo de $f$ contém o núcleo de $\omega$, i.e., se para um campo multivetorial $\xi$ de grau arbitrário, vale

$$
i_{\xi} \omega=0 \Longrightarrow i_{\xi} f=0 \text {. }
$$


Teorema 1.8 [12] Seja $P$ uma variedade multissimplética exata com n-forma multicanônica $\theta$ e $(n+1)$-forma multissimplética $\omega=-d \theta$ tal que $\theta$ seja uma forma de Poisson. Para quaisquer duas formas de Poisson $f$ de grau $n-r$ e g de grau $n-s$ sobre $P$, chamamos a forma de Poisson $\{f, g\}$ de grau $n-r-s+1$ dada por

$$
\begin{aligned}
\{f, g\}= & (-1)^{r(s-1)} i_{Y} i_{X} \omega \\
& +d\left((-1)^{(r-1)(s-1)} i_{Y} f-i_{X} g-(-1)^{(r-1) s} i_{Y} i_{X} \theta\right),
\end{aligned}
$$

onde $X$ e $Y$ são quaisquer dois campos multivetoriais hamiltonianos de graus $r$ e $s$ associados a $f$ e g, respectivamente, o colchete de Poisson de $f$ e g. Este colchete, além de bem definido e graduado antissimétrico, satisfaz a identidade de Jacobi graduada e prove o espaço das formas de Poisson sobre $P$ de uma estrutura de superálgebra de Lie.

Outra questão importante refere-se à escolha da variedade multissimplética $P$ a ser utilizada na construção do colchete de Poisson. Inicialmente, o problema foi analisado, por vários autores, no âmbito do espaço de multifase comum, mas muitas vezes usando construções em coordenadas locais que carecem de um significado geométrico intrínsico, e mais recentemente no âmbito do espaço de multifase estendido. No entanto, como foi discutido na Seção 1.5.1, dada uma hamiltoniana $\mathcal{H}$, o espaço de multifase comum $\vec{J}^{\circledast} E$ também é uma variedade exatamente multissimplética, com $n$-forma multicanônica $\theta_{\mathcal{H}}$ e $(n+1)$-forma multissimplética $\omega_{\mathcal{H}}=-d \theta_{\mathcal{H}}$, e portanto este também vem munido de um colchete de Poisson $\{\cdot, \cdot\}_{\mathcal{H}}$ que, obviamente, depende da hamiltoniana $\mathcal{H}$, conferindo-lhe uma característica dinâmica. Devido a esta distinção, referimo-nos ao colchete definido no espaço de multifase estendido $J^{\oplus} E$ como colchete cinemático e ao colchete definido no espaço de multifase comum $\vec{J}{ }^{\circledast} E$ como colchete dinâmico. 


\begin{tabular}{|c|c|}
\hline Mecânica não-autônoma & Teoria dos campos \\
\hline $\begin{array}{l}\text { espaço de configuração estendido } \\
\qquad \mathbb{R} \times Q \rightarrow \mathbb{R} \\
\text { coordenadas locais adaptadas } t, q^{i}\end{array}$ & $\begin{array}{l}\text { fibrado de configuração } \\
\qquad E \rightarrow M \\
\text { coordenadas locais adaptadas } x^{\mu}, q^{i}\end{array}$ \\
\hline $\begin{array}{l}\text { espaço de fase simplesmente estendido } \\
\qquad \mathcal{P}_{0}=\mathbb{R} \times T^{*} Q \\
\text { coordenadas locais adaptadas } t, q^{i}, p_{i}\end{array}$ & $\begin{array}{l}\text { espaço de multifase comum } \\
\qquad \mathcal{P}_{0}=\vec{J}^{\circledast} E=\vec{J}^{*} E \otimes \Lambda^{n} T^{*} M \\
\text { coordenadas locais adaptadas } x^{\mu}, q^{i}, p_{i}^{\mu}\end{array}$ \\
\hline $\begin{array}{l}\text { espaço de fase duplamente estendido } \\
\qquad \mathcal{P}=T^{*}(\mathbb{R} \times Q)=\mathbb{R} \times T^{*} Q \times \mathbb{R} \\
\text { coordenadas locais adaptadas } t, q^{i}, p_{i}, E\end{array}$ & $\begin{array}{l}\text { espaço de multifase estendido } \\
\qquad \mathcal{P}=J^{\circledast} E=J^{\star} E \otimes \bigwedge^{n} T^{*} M \\
\text { coordenadas locais adaptadas } x^{\mu}, q^{i}, p_{i}^{\mu}, p\end{array}$ \\
\hline $\begin{array}{l}\text { 1-forma canônica sobre } \mathcal{P} \\
\qquad \theta=p_{i} d q^{i}+E d t\end{array}$ & $\begin{array}{l}n \text {-forma multicanônica sobre } \mathcal{P} \\
\qquad \theta=p_{i}^{\mu} d q^{i} \wedge d^{n} x_{\mu}+p d^{n} x\end{array}$ \\
\hline $\begin{array}{l}\text { 2-forma simplética sobre } \mathcal{P} \text {, } \\
\text { não degenerada } \\
\qquad \omega=-d \theta=d q^{i} \wedge d p_{i}-d E \wedge d t\end{array}$ & $\begin{array}{l}\text { (n+1)-forma multissimplética sobre } \mathcal{P} \text {, } \\
\text { não degenerada (em campos vetoriais) } \\
\qquad \omega=-d \theta=d q^{i} \wedge d p_{i}^{\mu} \wedge d^{n} x_{\mu}-d p \wedge d^{n} x\end{array}$ \\
\hline $\begin{array}{l}\text { hamiltoniana } \\
\qquad \mathcal{H}: \mathcal{P}_{0} \rightarrow \mathbb{R} \\
\text { função sobre } \mathcal{P}_{0}\end{array}$ & $\begin{array}{l}\text { hamiltoniana } \\
\qquad \mathcal{H}: \mathcal{P}_{0} \rightarrow \mathcal{P} \\
\text { seção de } \mathcal{P} \text { sobre } \mathcal{P}_{0} \text { (fibrado afim em linhas) }\end{array}$ \\
\hline $\begin{array}{l}\text { Campo de Euler } \\
\qquad \Sigma=p_{i} \frac{\partial}{\partial p_{i}}+E \frac{\partial}{\partial E}\end{array}$ & $\begin{array}{l}\text { Campo de Euler } \\
\qquad \Sigma=p_{i}^{\mu} \frac{\partial}{\partial p_{i}^{\mu}}+p \frac{\partial}{\partial p}\end{array}$ \\
\hline
\end{tabular}

Tabela comparativa entre a geometria simplética na mecânica não-autônoma e a geometria multissimplética na teoria clássica dos campos. 


\section{Tensor de Poisson}

A nossa meta principal neste capítulo é investigar a possibilidade de construir, no âmbito do formalismo multissimplético, ou seja, sobre o espaço de multifase estendido $P=J^{\circledast} E$; um tensor de Poisson que desempenharia um papel semelhante ao tensor de Poisson na geometria simplética. Tendo em vista que a forma multissimplética $\omega$ é de grau $n+1$, é de se esperar que este tensor de Poisson $\pi$ deve ser um campo multivetorial de grau $n+1$. Obviamente, um campo multivetorial $\pi$ de grau $n+1$ induz, para todo $r$ com $0 \leqslant r \leqslant n$, um homomorfismo de fibrados vetoriais

$$
\pi: \bigwedge^{n-r+1} T^{*} P \longrightarrow \bigwedge^{r} T P
$$

assim generalizando o homomorfismo (5) da geometria simplética. A meta básica seria definir $\pi$ de tal forma que para formas $\alpha$ que se anulam sobre o núcleo de $\omega$, valha a relação

$$
i_{\pi(\alpha)} \omega=\alpha
$$

pois isto garante que

$$
X=\pi(d f)
$$

será uma solução da condição básica

$$
i_{X} \omega=d f
$$

que relaciona campos multivetoriais hamiltonianos e forma hamiltonianos.

Infelizmente, esta construção depende da escolha de certos dados adicionais, que são conexões em vários fibrados. Como veremos, dois tensores de Poisson construídos a partir de diferentes escolhas de conexões diferem por um campo multivetorial a valores no núcleo de $\omega$, o que reflete a ambiguidade na relação entre $X$ e $f$ que já enfrentamos no Capítulo 1 . Mesmo assim, é interessante identificar qual tipo de estrutura geométrica é suficiente para remover esta ambiguidade. 
Para descrever a referida construção, torna-se necessário aprofundar-se no estudo de conexões, tanto de conexões gerais em fibrados gerais como de conexões lineares em fibrados vetoriais e de conexões afins em fibrados afins. Será este o assunto que nos ocupará pela maior parte do presente capítulo.

Começaremos discutindo na primeira seção as várias definições da noção de conexão em um fibrado geral e como a escolha de uma conexão leva à definição de uma derivada covariante de seções. Nas duas seções seguintes faremos primeiro uma revisão do conceito de conexões lineares em fibrados vetoriais, passando em seguida ao conceito de conexões afins em fibrados afins. Também discutiremos a noção de conexão dual de uma conexão linear/afim em um fibrado vetorial/afim dado, que em ambos os casos é uma conexão linear no fibrado vetorial dual. Na quarta seção, estenderemos parte desta discussão a conexões parciais, definidas apenas ao longo de subfibrados do fibrado tangente $T M$ da variedade base $M$. Na quinta seção, consideraremos conexões compostas em fibrados compostos. Na sexta e na sétima seção, combinaremos os conceitos introduzidos anteriormente para mostrar como construir conexões lineares ou afins em vários fibrados vetoriais ou afins sobre o espaço total $E$ de um fibrado geral, tais como o fibrado vertical $V E$, o fibrado $J E$ dos jatos de primeira ordem e seu dual afim $J^{\oplus} E$, a partir de dados mais simples: uma conexão geral em $E$, uma conexão linear parcial em $V E$ ao longo de $V E$ e, nos últimos dois casos, uma conexão linear na variedade base $M$. Finalmente, na última seção, definiremos os referenciais de Darboux induzidos no fibrado $J^{\circledast} E$ pela conexão construída na seção anterior. Nestes referenciais definiremos o tensor de Poisson e ao final daremos algumas propriedades do tensor de Poisson.

\subsection{Conexões e derivadas covariantes}

Nesta seção apresentamos várias definições, todas equivalentes, do conceito de uma conexão geral em um fibrado geral $E$ sobre $M$, sem nenhuma estrutura adicional.

Definição 2.1 Uma conexão geral em um fibrado geral $E$ sobre $M$ é dada por uma seção global

$$
\Gamma: E \longrightarrow J E
$$

do fibrado afim $J E \longrightarrow E$.

Existe uma definição alternativa, mais tradicional, segundo a qual uma conexão geral em um fibrado geral $E$ sobre $M$ pode ser vista como um subfibrado vetorial $H E$ do fibrado tangente $T E$, chamado o fibrado horizontal, que é complementar ao fibrado vertical $V E$ :

$$
T E=\underline{H} E \oplus V E .
$$


A escolha de um fibrado horizontal equivale à escolha de uma cisão

$$
\pi^{*} T M \longrightarrow T E
$$

da seguinte sequência exata de fibrados vetoriais sobre $E$ :

$$
0 \longrightarrow V E \longrightarrow T E \longrightarrow \pi^{*} T M \longrightarrow 0
$$

Ainda equivale à escolha de uma projeção horizontal $H: T E \rightarrow T E$ ou de uma projeção vertical $V: T E \rightarrow T E$, que são homomorfismos de fibrados vetoriais sobre $E$ tais que $H^{2}=H, V^{2}=V, V E=\operatorname{im}(V)=\operatorname{ker}(H), H E=\operatorname{ker}(V)=\operatorname{im}(H)$ e $H+V=\mathrm{id}_{T E}$.

Para mostrar que as duas definições dadas acima são de fato equivalentes, lembramos que uma seção global $\Gamma$ de $J E$ sobre $E$ associa a cada ponto $e \in E$ sobre um ponto $x \in M$ um elemento $\Gamma(e) \in L\left(T_{x} M, T_{e} E\right)$ tal que $T_{e} \pi \circ \Gamma(e)=\operatorname{id}_{T_{x} M}$. Mas então $V_{e} E \cap \operatorname{im}(\Gamma(e))=\{0\}$ e $\operatorname{ker}(\Gamma(e))=\{0\}$. A última relação implica que im $(\Gamma(e))$ e $T_{x} M$ têm a mesma dimensão, e como $T_{e} \pi$ é sobrejetora, segue $V_{e} E \oplus \operatorname{im}(\Gamma(e))=T_{e} E$, ou seja, $\operatorname{im}(\Gamma(e))$ é subespaço horizontal de $T_{e} E$. Assim, uma seção global de $J E$ determina um fibrado horizontal de $E$. Reciprocamente, a partir de um subespaço horizontal $H_{e} E$ de $T_{e} E$, obtemos um elemento de $J_{e} E$ considerando a inversa da restrição de $T_{e} \pi$ a $H_{e} E$. Assim um fibrado horizontal de $E$ determina uma seção global de $J E$.

Uma outra forma de estabelecer esta bijeção é através da cisão (2.7), que equivale a uma seção do fibrado vetorial $\pi^{*} T^{*} M \otimes T E$ sobre $E$, pois este contém $J E$ como subfibrado afim.

Dada uma conexão geral $\Gamma$ no fibrado geral $E$, podemos para todo campo vetorial $X_{M}$ sobre $M$ introduzir seu levantamento horizontal, que é um campo vetorial $\Gamma\left(X_{M}\right)$ sobre $E$ definido por

$$
\Gamma\left(X_{M}\right)(e)=\Gamma(e) \cdot X_{M}(x)
$$

para todo $e \in E$ sobre $x \in M$. É fácil mostrar que para quaisquer dois campos vetoriais $X_{M}$ e $Y_{M}$ sobre $M$, a expressão

$$
\Omega\left(X_{M}, Y_{M}\right)=\left[\Gamma\left(X_{M}\right), \Gamma\left(Y_{M}\right)\right]-\Gamma\left(\left[X_{M}, Y_{M}\right]\right)
$$

representa um campo vetorial vertical sobre $E$. Mais geralmente, a forma de curvatura da conexão $\Gamma$ é definida como a 2 -forma horizontal $\Omega$ sobre $E$ a valores no fibrado vertical $V E$ tal que para dois campos vetoriais $X$ e $Y$ sobre $E, \Omega(X, Y)$ é a parte vertical do colchete de Lie entre as partes horizontais dos campos $X$ e $Y$ :

$$
\Omega(X, Y)=\left[X_{H}, Y_{H}\right]_{V}
$$

Portanto, a forma de curvatura $\Omega$ mede quanto o fibrado horizontal deixa de ser involutivo. 
Em coordenadas locais, escrevemos a seção (2.5) na forma

$$
\Gamma(x, q)=\left(x^{\mu}, q^{i} ; \Gamma_{\mu}^{i}(x, q)\right) .
$$

Outra representação, que enfatiza a interpretação de uma conexão como fornecendo uma base local de 1 -formas horizontais a valores no fibrado tangente $T E$ de $E$, é a seguinte [28]

$$
\Gamma=d x^{\mu} \otimes\left(\partial_{\mu}+\Gamma_{\mu}^{i} \partial_{i}\right)
$$

Isto significa que o levantamento horizontal do campo local $\partial_{\mu}$ sobre $M$ é o campo local $\Gamma\left(\partial_{\mu}\right)$ sobre $E$ dado por

$$
\Gamma\left(\partial_{\mu}\right)=\partial_{\mu}+\Gamma_{\mu}^{i} \partial_{i} .
$$

Nos mesmos termos, a projeção vertical $V$ e a projeção horizontal $H$ são dadas por

$$
\begin{aligned}
& V(x, q ; \dot{x}, \dot{q})=\left(x^{\mu}, q^{i} ; 0, \dot{q}^{i}+\Gamma_{\mu}^{i}(x, q) \dot{x}^{\mu}\right), \\
& H(x, q ; \dot{x}, \dot{q})=\left(x^{\mu}, q^{i} ; \dot{x}^{\mu},-\Gamma_{\mu}^{i}(x, q) \dot{x}^{\mu}\right) .
\end{aligned}
$$

Para a forma de curvatura $\Omega$ da conexão $\Gamma$, temos

$$
\Omega(X, Y)=X^{\mu} Y^{\nu} \Omega_{\mu \nu}^{i} \partial_{i}
$$

com

$$
\Omega_{\mu \nu}^{i}(x, q)=\partial_{\mu} \Gamma_{\nu}^{i}-\partial_{\nu} \Gamma_{\mu}^{i}+\Gamma_{\mu}^{j} \partial_{j} \Gamma_{\nu}^{i}-\Gamma_{\nu}^{j} \partial_{j} \Gamma_{\mu}^{i} .
$$

A lei de transformação para os coeficientes de conexão sob mudança de coordenadas locais como em $(1.22)$ e (1.24) é $\Gamma_{\mu}^{i} \rightarrow \Gamma_{\kappa}^{k}$, onde

$$
\Gamma_{\kappa}^{\prime k}=\frac{\partial x^{\mu}}{\partial x^{\prime \kappa}} \frac{\partial q^{\prime k}}{\partial q^{i}} \Gamma_{\mu}^{i}+\frac{\partial x^{\mu}}{\partial x^{\prime \kappa}} \frac{\partial q^{\prime k}}{\partial x^{\mu}} .
$$

Fixando uma conexão, podemos introduzir a noção de derivada covariante de seções, que generaliza a noção de derivada direcional de funções em $\mathbb{R}^{n}$. Em fibrados gerais, a derivada covariante determina a parte vertical da derivada comum: dada uma seção (local) $\varphi$ de $E$ sobre $M$, sua derivada comum é a seção (local) $j \varphi$ de $J E$ sobre $M$ (que corresponde à aplicação tangente $T \varphi: T M \rightarrow T E$ ) e a sua derivada covariante é uma seção (local) $\nabla^{\Gamma} \varphi$ de $T^{*} M \otimes \varphi^{*}(V E)$ sobre $M$, obtida por composição

$$
\nabla^{\Gamma} \varphi=V_{\Gamma} \circ j \varphi
$$

onde

$$
V_{\Gamma}: J E \longrightarrow \pi^{*}\left(T^{*} M\right) \otimes V E
$$


é a restrição a $J E$ de id $_{T^{*} M} \otimes V: T^{*} M \otimes T E \longrightarrow T^{*} M \otimes V E$. A relação com a idéia de derivada direcional é estabelecida por contração com campos vetoriais $X$ sobre $M$ : denotase por $\nabla_{X}^{\Gamma} \varphi$ a derivada covariante de $\varphi$ ao longo de $X$, que é uma seção de $\varphi^{*}(V E)$, com dependência $\mathfrak{F}(M)$-linear em $X$ :

$$
\nabla_{f X}^{\Gamma} \varphi=f \nabla_{X}^{\Gamma} \varphi
$$

Em coordenadas locais,

$$
V_{\Gamma}=\left(q_{\mu}^{i}-\Gamma_{\mu}^{i}\right) d x^{\mu} \otimes \partial_{i}
$$

enquanto que

$$
\nabla^{\Gamma} \varphi=\left(\partial_{\mu} \varphi^{i}-(\Gamma \circ \varphi)_{\mu}^{i}\right) d x^{\mu} \otimes \partial_{i}
$$

e

$$
\nabla_{X}^{\Gamma} \varphi=\left(\partial_{\mu} \varphi^{i}-(\Gamma \circ \varphi)_{\mu}^{i}\right) X^{\mu} \partial_{i}
$$

Escrevemos simplesmente $\nabla$ no lugar de $\nabla^{\Gamma}$, sempre que não houver perigo de confusão.

Reciprocamente, podemos perguntar quais seriam as condições a serem exigidas para que um operador $\nabla$ que leva cada seção $\varphi$ de $E$ para uma seção $\nabla \varphi$ de $T^{*} M \otimes \varphi^{*}(V E)$ seja a derivada covariante associada a uma conexão $\Gamma$ em $E$. Em geral, essas condições são complicadas e de difícil formulação. Por outro lado, são bastante simples no caso de conexões lineares em fibrados vetoriais ou conexões afins em fibrados afins, que passamos a considerar nas seguintes duas seções.

\subsection{Conexões lineares e duais lineares}

Definição 2.2 Uma conexão $\Gamma$ em um fibrado vetorial E sobre $M$ é dita ser uma conexão linear se a seção (2.5) for um homomorfismo de fibrados vetoriais sobre $M$.

Em coordenadas locais lineares ao longo das fibras, esta condição significa que

$$
\Gamma_{\mu}^{i}(x, q)=-A_{\mu j}^{i}(x) q^{j}
$$

Nestas coordenadas, a forma de curvatura tem a expressão

$$
\Omega_{\mu \nu}^{i}(x, q)=-F_{\mu \nu j}^{i}(x) q^{j}
$$

onde

$$
F_{\mu \nu j}^{i}=\partial_{\mu} A_{\nu j}^{i}-\partial_{\nu} A_{\mu j}^{i}+A_{\mu k}^{i} A_{\nu j}^{k}-A_{\nu k}^{i} A_{\mu j}^{k}
$$


A lei de transformação para os coeficientes $A_{\mu j}^{i}$ e os coeficientes $F_{\mu \nu j}^{i}$, conhecidos como potenciais de calibre e como campos de calibre, respectivamente, sob uma mudança de coordenadas locais como em (1.22) e (1.25) é $A_{\mu j}^{i} \rightarrow A_{\kappa l}^{\prime k}$ e $F_{\mu \nu j}^{i} \rightarrow F_{\kappa \lambda l}^{\prime k}$, onde

$$
\begin{gathered}
A_{\kappa l}^{\prime k}=\frac{\partial x^{\mu}}{\partial x^{\prime \kappa}}\left(g_{i}^{k} A_{\mu j}^{i}\left(g^{-1}\right)_{l}^{j}-\partial_{\mu} g_{i}^{k}\left(g^{-1}\right)_{l}^{i}\right), \\
F_{\kappa \lambda l}^{\prime k}=\frac{\partial x^{\mu}}{\partial x^{\prime \kappa}} \frac{\partial x^{\nu}}{\partial x^{\prime \lambda}} g_{i}^{k} F_{\mu \nu j}^{i}\left(g^{-1}\right)_{l}^{j} .
\end{gathered}
$$

Usando o fato de que $E$ sendo um fibrado vetorial, seu fibrado vertical $V E$ é canonicamente isomorfo a $\pi^{*} E$ e portanto $\varphi^{*}(V E) \cong E$ para qualquer seção $\varphi$ de $E$, podemos neste caso interpretar a derivada covariante como um operador diferencial linear

$$
\nabla: \Gamma(E) \longrightarrow \Gamma\left(T^{*} M \otimes E\right)
$$

de primeira ordem que satisfaz a regra de Leibniz, afirmando que para $f \in \mathfrak{F}(M), \varphi \in \Gamma(E)$ e $X \in \mathfrak{X}(M)$,

$$
\nabla_{X}(f \varphi)=(X \cdot f) \varphi+f \nabla_{X} \varphi .
$$

Reciprocamente, é bem conhecido que qualquer operador diferencial deste tipo é a derivada covariante associada a uma conexão linear. Em coordenadas locais lineares ao longo das fibras, temos $\nabla_{X} \varphi=X^{\mu} \nabla_{\mu} \varphi^{i} \partial_{i}$ com

$$
\nabla_{\mu} \varphi^{i}=\partial_{\mu} \varphi^{i}+A_{\mu j}^{i} \varphi^{j} .
$$

Conexões lineares comportam-se naturalmente sob o processo de dualização. Dado um fibrado vetorial $E$ sobre $M$, seja $E^{*}$ o seu dual linear, definido fibra por fibra: $\left(E^{*}\right)_{x}=\left(E_{x}\right)^{*}$. Qualquer conexão linear $\Gamma$ em $E$ induz uma conexão linear $\Gamma^{*}$ em $E^{*}$ cuja definição é mais facilmente dada em termos das correspondentes derivadas covariantes. De fato, usando o pareamento canônico

$$
\langle., .\rangle: E^{*} \times E \longrightarrow M \times \mathbb{R}
$$

entre $E$ e $E^{*}$, que induz um pareamento canônico

$$
\langle., .\rangle: \Gamma\left(E^{*}\right) \times \Gamma(E) \longrightarrow \mathfrak{F}(M)
$$

entre suas seções, os operadores $\nabla: \Gamma(E) \longrightarrow \Gamma\left(T^{*} M \otimes E\right)$ e $\nabla^{*}: \Gamma\left(E^{*}\right) \longrightarrow \Gamma\left(T^{*} M \otimes E^{*}\right)$ de derivada covariante em $E$ e em $E^{*}$ são determinados um pelo outro através da regra de Leibniz, afirmando que para $X \in \mathfrak{X}(M), \varphi \in \Gamma(E)$ e $\varphi^{*} \in \Gamma\left(E^{*}\right)$;

$$
X \cdot\left\langle\varphi^{*}, \varphi\right\rangle=\left\langle\nabla_{X}^{*} \varphi^{*}, \varphi\right\rangle+\left\langle\varphi^{*}, \nabla_{X} \varphi\right\rangle .
$$


Em coordenadas locais lineares ao longo das fibras, é conveniente denotar as coordenadas linearẹs verticais de $E$ por $q^{i}$ e as coordenadas lineares verticais de $E^{*}$ por $p_{i}$; então se a conexão linear original $\Gamma$ em $E$ é dada pela fórmula (2.25), a conexão dual $\Gamma^{*}$ em $E^{*}$ é dada por

$$
\left(\Gamma^{*}\right)_{\mu, i}(x, p)=A_{\mu i}^{j}(x) p_{j} .
$$

\subsection{Conexões afins e duais afins}

Definição 2.3 Uma conexão $\Gamma$ em um fibrado afim $E$ sobre $M$ é dita ser uma conexão afim se a seção (2.5) for um homomorfismo de fibrados afins sobre $M$.

Em coordenadas locais afins ao longo das fibras, esta condição significa que

$$
\Gamma_{\mu}^{i}(x, q)=-A_{\mu j}^{i}(x) q^{j}-A_{\mu}^{i}(x) \text {. }
$$

Nestas coordenadas, a forma de curvatura tem a expressão

$$
\Omega_{\mu \nu}^{i}(x, q)=-F_{\mu \nu j}^{i}(x) q^{j}-F_{\mu \nu}^{i}(x)
$$

onde

$$
F_{\mu \nu j}^{i}=\partial_{\mu} A_{\nu j}^{i}-\partial_{\nu} A_{\mu j}^{i}+A_{\mu k}^{i} A_{\nu j}^{k}-A_{\nu k}^{i} A_{\mu j}^{k}
$$

$\mathrm{e}$

$$
F_{\mu \nu}^{i}=\partial_{\mu} A_{\nu}^{i}-\partial_{\nu} A_{\mu}^{i}+A_{\mu k}^{i} A_{\nu}^{k}-A_{\nu k}^{i} A_{\mu}^{k} .
$$

A lei de transformação para os coeficientes $A_{\mu j}^{i}$ e $A_{\mu}^{i}$ e os coeficientes $F_{\mu \nu j}{ }^{i}$ e $F_{\mu \nu}^{i}$, conhecidos como potenciais de calibre e como campos de calibre, respectivamente, sob uma mudança de coordenadas locais como em (1.22) e (1.26) é $A_{\mu j}^{i} \rightarrow A_{\kappa l}^{\prime k}$ e $A_{\mu}^{i} \rightarrow A_{\kappa}^{\prime k}, F_{\mu \nu j}^{i} \rightarrow F_{\kappa \lambda l}^{\prime k}$ e $F_{\mu \nu}^{i} \rightarrow F_{\kappa \lambda}^{\prime k}$, onde

$$
\begin{gathered}
A_{\kappa l}^{\prime k}=\frac{\partial x^{\mu}}{\partial x^{\prime \kappa}}\left(g_{i}^{k} A_{\mu j}^{i}\left(g^{-1}\right)_{l}^{j}-\partial_{\mu} g_{i}^{k}\left(g^{-1}\right)_{l}^{i}\right) \\
A_{\kappa}^{\prime k}=\frac{\partial x^{\mu}}{\partial x^{\prime \kappa}}\left(g_{i}^{k} A_{\mu}^{i}-\partial_{\mu} g^{k}-g_{i}^{k} A_{\mu j}^{i}\left(g^{-1}\right)_{l}^{j} g^{l}+\partial_{\mu} g_{i}^{k}\left(g^{-1}\right)_{l}^{i} g^{l}\right) \\
F_{\kappa \lambda l}^{\prime k}=\frac{\partial x^{\mu}}{\partial x^{\prime \kappa}} \frac{\partial x^{\nu}}{\partial x^{\prime \lambda}} g_{i}^{k} F_{\mu \nu j}{ }^{i}\left(g^{-1}\right)_{l}^{j} \\
F_{\kappa \lambda}^{\prime k}=\frac{\partial x^{\mu}}{\partial x^{\prime \kappa}} \frac{\partial x^{\nu}}{\partial x^{\prime \lambda}}\left(g_{i}^{k} F_{\mu \nu}{ }^{i}-g_{i}^{k} F_{\mu \nu j}{ }^{i}\left(g^{-1}\right)_{l}^{j} g^{l}\right) .
\end{gathered}
$$


Toda conexão afim $\Gamma$ em um fibrado afim $E$ sobre $M$ induz uma conexão linear $\vec{\Gamma}$ no seu fibrado de diferenças $\vec{E}$, sendo que o homomorfismo

$$
\vec{\Gamma}: \vec{E} \longrightarrow J_{M} \vec{E}
$$

de fibrados vetoriais sobre $M$ é simplesmente a parte linear do homomorfismo

$$
\Gamma: E \longrightarrow J_{M} E
$$

de fibrados afins sobre $M$. Em coordenadas locais vetoriais de $\vec{E}$ ao longo das fibras (provindo de coordenadas locais afins de $E$ ao longo das fibras),

$$
\vec{\Gamma}_{\mu}^{i}(x, q)=-A_{\mu j}^{i}(x) q^{j} .
$$

Usando o fato de que $E$ sendo um fibrado afim, seu fibrado vertical $V E$ é canonicamente isomorfo a $\pi^{*} \vec{E}$ e portanto $\varphi^{*}(V E) \cong \vec{E}$ para qualquer seção $\varphi$ de $E$, podemos neste caso interpretar a derivada covariante associada a $\Gamma$ como um operador diferencial afim

$$
\nabla: \Gamma(E) \longrightarrow \Gamma\left(T^{*} M \otimes \vec{E}\right)
$$

de primeira ordem e a derivada covariante associada a $\vec{\Gamma}$ como um operador diferencial linear

$$
\vec{\nabla}: \Gamma(\vec{E}) \longrightarrow \Gamma\left(T^{*} M \otimes \vec{E}\right)
$$

de primeira ordem, satisfazendo a regra de Leibniz, relacionados por

$$
\vec{\nabla}_{X}(\varphi-\psi)=\nabla_{X} \varphi-\nabla_{X} \psi
$$

Reciprocamente, pode-se mostrar que qualquer operador diferencial deste tipo é a derivada covariante associada a uma conexão afim. Em coordenadas locais afins ao longo das fibras de $E$, temos $\nabla_{X} \varphi=X^{\mu} \nabla_{\mu} \varphi^{i} \partial_{i}$ com

$$
\nabla_{\mu} \varphi^{i}=\partial_{\mu} \varphi^{i}+A_{\mu j}^{i} \varphi^{j}+A_{\mu}^{i} .
$$

Em seguida, faremos uso do fato de que sempre podemos, a partir de qualquer conexão linear $\vec{\Gamma}$ em $\vec{E}$ e qualquer seção $\varphi_{0}$ de $E$, construir uma única conexão afim $\Gamma$ em $E$ que tenha $\vec{\Gamma}$ como parte linear e tal que a seção $\varphi_{0}$ seja covariantemente constante:

$$
\nabla^{\Gamma} \varphi_{0}=0 \text {. }
$$

Em coordenadas locais afins ao longo das fibras de $E$, isso significa que

$$
A_{\mu}^{i}=-\left(\partial_{\mu} \varphi_{0}^{i}+A_{\mu j}^{i} \varphi_{0}^{j}\right)
$$


Finalmente, conexões afins também se comportam naturalmente sob o processo de dualização. Dado um fibrado afim $E$ sobre $M$, seja $E^{*}$ o seu dual afim, definido fibra por fibra: $\left(E^{\star}\right)_{x}=\left(E_{x}\right)^{\star}$. Qualquer conexão afim $\Gamma \mathrm{em} E$ induz uma conexão linear $\Gamma^{\star} \mathrm{em} E^{\star}$ cuja definição é mais facilmente dada em termos das correspondentes derivadas covariantes. De fato, usando os pareamentos canônicos

$$
\langle., .\rangle: E^{\star} \times E \longrightarrow M \times \mathbb{R}
$$

entre $E^{\star}$ e $E$ e

$$
\langle., .\rangle: E^{\star} \times \vec{E} \longrightarrow M \times \mathbb{R}
$$

entre $E^{\star}$ e $\vec{E}$, que induzem pareamentos canônicos

$$
\langle., .\rangle: \Gamma\left(E^{*}\right) \times \Gamma(E) \longrightarrow \mathfrak{F}(M)
$$

e

$$
\langle., .\rangle: \Gamma\left(E^{*}\right) \times \Gamma(\vec{E}) \longrightarrow \mathfrak{F}(M)
$$

entre suas seções, os operadores $\nabla: \Gamma(E) \longrightarrow \Gamma\left(T^{*} M \otimes \vec{E}\right)$ e $\nabla^{*}: \Gamma\left(E^{\star}\right) \longrightarrow \Gamma\left(T^{*} M \otimes E^{*}\right)$ de derivada covariante em $E$ e em $E^{*}$ são determinados um pelo outro através da regra de Leibniz, afirmando que para $X \in \mathfrak{X}(M), \varphi \in \Gamma(E)$ e $\varphi^{\star} \in \Gamma\left(E^{\star}\right)$,

$$
X \cdot\left\langle\varphi^{\star}, \varphi\right\rangle=\left\langle\nabla_{X}^{*} \varphi^{\star}, \varphi\right\rangle+\left\langle\varphi^{\star}, \nabla_{X} \varphi\right\rangle .
$$

Em coordenadas locais afins/lineares ao longo das fibras, é conveniente denotar as coordenadas afins verticais de $E$ por $q^{i}$, as coordenadas lineares verticais de $\vec{E}$ por $\vec{q}^{i}$ e as coordenadas lineares verticais de $E^{*}$ por $\left(p_{i}, p_{0}\right)$, de forma que o pareamento (2.54) é dado por $\langle p, q\rangle=p_{i} q^{i}+p_{0}$ e o pareamento (2.55) por $\langle p, \vec{q}\rangle=p_{i} \vec{q}^{i}$; então se a conexão afim original $\Gamma$ em $E$ é dada pela fórmula (2.37), a conexão dual $\Gamma^{\star}$ em $E^{\star}$ é dada por

$$
\left(\Gamma^{\star}\right)_{\mu, i}(x, p)=A_{\mu i}^{j}(x) p_{j}, \quad\left(\Gamma^{\star}\right)_{\mu, 0}(x, p)=A_{\mu}^{i}(x) p_{i} .
$$

\subsection{Conexões parciais}

O conceito de conexão parcial nasceu no contexto de conexões lineares, representadas pelos correspondentes operadores de diferenciação covariante, admitindo-se que seções possam possuir derivadas covariantes apenas ao longo de certas direções. As direções permitidas são representadas pela escolha de um subfibrado vetorial $D$ do fibrado tangente $T M$ da variedade base $M$. A situação mais importante ocorre quando este subfibrado for involutivo.

Para estender o conceito de uma conexão parcial de conexões lineares a conexões gerais, introduzimos o conceito do fibrado dos jatos parciais de primeira ordem associado a um 
fibrado geral $E$ e um subfibrado vetorial $D$ de sua variedade base, denotado aqui por $J$ " $E$. Dado um fibrado $E$ sobre $M$ com projeção $\pi$ e fibra típica $Q$ e um subfibrado vetorial $D$ de $T M$, a fibra de $J " E$ em cada ponto $e \in E$ sobre o ponto $x \in M$ é o espaço afim

$$
J_{e}^{\|} E=\left\{u \in L\left(D_{x}, T_{e} E\right): T_{e} \pi \circ u=\mathrm{id}_{D_{x}}\right\},
$$

que é um subespaço afịm do espaço vetorial $L\left(D_{x}, T_{e} E\right)$, com espaço modelo $L\left(D_{x}, V_{e} E\right)$. Deixando $e$ variar sobre $E$, obtemos um fibrado afim sobre $E$ em relação à projeção alvo $\tau^{\|}: J^{\|} E \rightarrow E$, que é um subfibrado do fibrado vetorial $L\left(\pi^{*} D, T E\right) \cong \pi^{*} D^{*} \otimes T E$. Novamente, os pontos de $\bar{J} \| E$ podem ser representados como 1 -jatos de seções (locais) de $E$ : para todo ponto $e \in E$ sobre o ponto $x \in M$, os elementos de $J_{e}^{\|} E$ podem ser escritos na forma $u=j_{x}^{\|} \varphi$ onde $\varphi$ é uma seção de $E$ definida em uma vizinhança aberta de $x$ em $M$ tal que $\varphi(x)=e$ e $\left.T_{x} \varphi\right|_{D_{x}}=u$.

Definição 2.4Uma conexão parcial geral em um fibrado geral $E$ sobre $M$, ao longo de um subfibrado vetorial $D$ de TM, é dada por uma seção global

$$
\Gamma^{\|}: E \longrightarrow J^{\|} E
$$

do fibrado afim $J^{\|} E \longrightarrow E$.

Como no caso de conexões usuais, existe uma definição alternativa, cuja formulação requer substituir o fibrado tangente $T E$ ao espaço total pelo subfibrado vetorial

$$
T_{D} E=(T \pi)^{-1}(D) \text {. }
$$

De fato, uma conexão parcial geral em um fibrado geral $E$ sobre $M$, ao longo de um subfibrado vetorial $D$ de $T M$, pode ser vista como um subfibrado vetorial $H_{D} E$ do fibrado $T_{D} E$, chamado o fibrado horizontal parcial, que é complementar ao fibrado vertical $V E$ :

$$
T_{D} E=H_{D} E \oplus V E
$$

A escolha de um fibrado horizontal parcial equivale à escolha de uma cisão

$$
\pi^{*} D \longrightarrow T_{D} E
$$

da seguinte sequência exata de fibrados vetoriais sobre $E$ :

$$
0 \longrightarrow V E \longrightarrow T_{D} E \longrightarrow \pi^{*} D \longrightarrow 0
$$

Ainda equivale à escolha de uma projeção horizontal parcial $H: T_{D} E \rightarrow T_{D} E$ ou de uma projeção vertical parcial $V: T_{D} E \rightarrow T_{D} E$, que são homomorfismos de fibrados vetoriais 
sobre $E$ tais que $H^{2}=H, V^{2}=V, V E=\operatorname{im}(V)=\operatorname{ker}(H), H_{D} E=\operatorname{ker}(V)=\operatorname{im}(H)$ e $H+V=\mathrm{id}_{T_{D} E}$.

A equivalência entre as duas definições se demonstra da mesma forma como no caso de conexões usuais. Também é claro que dada uma conexão parcial $\Gamma^{\|}$em $E$ ao longo de $D$ podemos definir o levantamento horizontal $\Gamma^{\|}\left(X_{M}\right)$ de qualquer campo vetorial $X_{M}$ sobre $M$ tangente a $D$, substituindo $\Gamma$ por $\Gamma^{\| l}$ na expressão em (2.9). No entanto, a definição de curvatura requer como hipótese que $D$ seja involutivo, o que implica que $T_{D} E$ também é involutivo. Neste caso, observa-se que a expressão em (2.10), com $\Gamma$ substituído por $\Gamma^{\|}$, continua bem definida, para quaisquer dois campos vetoriais $X_{M}$ e $Y_{M}$ sobre $M$ tangentes a $D$, e que a expressão em (2.11) continua bem definida, para quaisquer duas seções $X$ e $Y$ de $T_{D} E$.

Para uma distribuição integrável $D$ de dimensão $d$, podemos considerar sistemas de coordenadas locais em $M$ adaptados a esta distribuição

$$
\left(x_{\|}^{\mu}, x_{\sharp}^{\mu}\right)=\left(x^{\mu_{1}}, \ldots, x^{\mu_{d}}, x^{\mu_{d+1}}, \ldots, x^{\mu_{n}}\right) \text {, }
$$

de tal maneira que as variedades integrais maximais de $D$ são descritas por

$$
x_{\sharp}^{\mu}=\text { const } .
$$

Esta decomposição das coordenadas locais de $M$ em "coordenadas paralelas" e "coordenadas transversais" induz uma decomposição correspondente para os sistemas de coordenadas locais de $E$ e de $J E$ introduzidos anteriormente, conforme $\left(x_{\|}^{\mu}, x_{\sharp}^{\mu} ; q^{i}\right)$ e $\left(x_{\|}^{\mu}, x_{\sharp}^{\mu} ; q^{i}, q_{\mu \|}^{i}, q_{\mu \sharp}^{i}\right)$, respectivamente; então as coordenadas locais induzidas de $J \| E$ são $\left(x_{\|}^{\mu}, x_{\sharp}^{\mu} ; q^{i}, q_{\mu \|}^{i}\right)$, e a seção (2.61) assume a forma

$$
\Gamma^{\|}(x, q)=\left(x_{\|}^{\mu}, x_{\sharp}^{\mu}, q^{i} ; \Gamma_{\mu \|}^{i}(x, q)\right),
$$

ou na interpretação de soma de 1-formas horizontais a valores no fibrado tangente $T E$ de $E$,

$$
\Gamma^{\|}=d x_{\|}^{\mu} \otimes\left(\partial_{\mu \|}+\Gamma_{\mu \|}^{i} \partial_{i}\right)
$$

Em geral, a distribuição $D$ não admite nenhuma distribuição complementar privilegiada. No entanto, podemos perguntar o que acontece quando fixarmos um subfibrado vetorial $D^{\prime}$ de $T M$ complementar ao subfibrado vetorial original $D$ de $T M$ :

$$
T M=D \oplus D^{\prime} .
$$

Neste caso, é fácil ver que a escolha de um par $\left(\Gamma^{\| \prime}, \Gamma^{\| \prime}\right)$ de conexões parciais em $E, \Gamma^{\|}$ao longo de $D$ e $\Gamma^{\prime \prime \prime}$ ao longo de $D^{\prime}$, corresponde exatamente à escolha de uma conexão comum $\Gamma$ em $E$, sendo que

$$
T E=H_{D} E \oplus H_{D^{\prime}} E \oplus V E
$$


Também podemos afirmar que

$$
\Gamma=\Gamma^{\| \prime} \oplus \Gamma^{\| \prime \prime}
$$

já que vale

$$
J E=J^{\|} E \oplus J^{\|^{\prime}} E .
$$

Em particular, quando $D^{\prime}$ for o complemento ortogonal de $D$ em relação a alguma métrica, usamos o símbolo.$^{\perp}$, ao invés de. ${ }^{\prime \prime}$. Quanto ao uso de coordenadas locais, podemos no caso dos dois subfibrados, $D$ e $D^{\prime}$, serem integráveis, escolher as coordenadas transversais a $D$ tais que sejam coordenadas ao longo de $D^{\prime}$.

Como no caso do fibrado $J E$, estruturas adicionais sobre o fibrado $E$ podem ser herdadas pelo fibrado $J \| E$. Em particular, prova-se da mesma forma que antes (substituindo $j_{x} \varphi$ por $j_{x}^{\|} \varphi$ ) que se $E$ for um fibrado vetorial/afim sobre $M$ então $J \| E$ também será um fibrado vetorial/afim sobre $M$.

Definição 2.5 Uma conexão parcial $\Gamma^{\| l}$ em um fibrado vetorial/afim $E$ sobre $M$ é dita ser uma conexão parcial linear/afim se a seção (2.61) for um homomorfismo de fibrados vetoriais/afins sobre $M$.

No caso linear, que é o caso de interesse a seguir, usando um sistema de coordenadas locais lineares a.o longo das fibras, adaptado à distribuição $D$, temos

$$
\Gamma_{\mu \|}^{i}(x, q)=-A_{\mu \| j}^{i}(x) q^{j} .
$$

Nestas coordenadas, a forma de curvatura tem a expressão

$$
\Omega_{\mu \nu \|}^{i}(x, q)=-F_{\mu \nu \| j}^{i}(x) q^{j} .
$$

Finalmente, podemos introduzir o conceito de derivada covariante associada a uma conexão parcial linear como um operador diferencial linear

$$
\nabla^{\|}: \Gamma(E) \longrightarrow \Gamma\left(D^{*} \otimes E\right)
$$

de primeira ordem que satisfaz a regra de Leibniz, afirmando que para $f \in \mathfrak{F}(M), \varphi \in \Gamma(E)$ e $X \in \mathfrak{X}(M)$ tangente a $D$,

$$
\nabla_{X}^{\|}(f \varphi)=(X \cdot f) \varphi+f \nabla_{X}^{\|} \varphi .
$$

Reciprocamente, qualquer operador diferencial deste tipo é a derivada covariante associada a uma conexão parcial linear. Em coordenadas locais lineares ao longo das fibras, adaptadas à distribuição $D$, temos $\nabla_{X}^{\|} \varphi=X^{\mu} \nabla_{\mu}^{\|} \varphi^{i} \partial_{i} \operatorname{com}$

$$
\nabla_{\mu}^{\|} \varphi^{i}=\partial_{\mu \|} \varphi^{i}+A_{\mu \| j}^{i} \varphi^{j}
$$

Desta forma vemos que $D$ representa as "direções permitidas", ao longo das quais seções possuem derivadas covariantes. 


\subsection{Conexões em fibrados compostos}

Nesta seção estudamos conexões em fibrados compostos. Considere um fibrado $F \stackrel{\rho}{\longrightarrow} E$ cuja base é o espaço total de outro fibrado $E \stackrel{\pi}{\longrightarrow} M$; então obtemos um terceiro fibrado $F \stackrel{\pi \circ \rho}{\longrightarrow} M$, chamado de fibrado composto. (Exemplos seriam $F=V E$ ou $F=J E$.) No que segue, queremos estabelecer diversos tipos de relações entre conexões $\Gamma_{E}$ em $E, \hat{\Gamma}_{F}$ em $F$ como fibrado sobre $M$ e $\tilde{\Gamma}_{F}$ em $F$ como fibrado sobre $E$.

Como pré-requisito usamos as respectivas projeções alvo para definir os fibrados verticais $V E \stackrel{\tau_{E}}{\longrightarrow} E, V_{E} F \stackrel{\tau_{F, E}}{\longrightarrow} F$ (obtido a partir de $F \stackrel{\rho}{\longrightarrow} E$ ) e $V_{M} F \stackrel{\tau_{F, M}}{\longrightarrow} M$ (obtido a partir de $F \stackrel{\pi \circ \rho}{\longrightarrow} M)$. Em coordenadas locais, estes fibrados são parametrizados, respectivamente, por

$$
\left(x^{\mu}, q^{i} ; \dot{q}^{i}\right),\left(x^{\mu}, q^{i}, r^{m} ; \dot{r}^{m}\right),\left(x^{\mu}, q^{i}, r^{m} ; \dot{q}^{i}, \dot{r}^{m}\right) .
$$

Com notação similar para $J$ no lugar de $V$, podemos definir os fibrados de jatos $J E \stackrel{\tau_{E}}{\longrightarrow} E$, $J_{E} F \stackrel{\tau_{F, E}}{\longrightarrow} F$ (obtido a partir de $F \stackrel{\rho}{\longrightarrow} E$ ) e $J_{M} F \stackrel{\tau_{F, M}}{\longrightarrow} M$ (obtido a partir de $F \stackrel{\pi \circ \rho}{\longrightarrow} M$ ). Em coordenadas locais, estes fibrados são parametrizados, respectivamente, por

$$
\left(x^{\mu}, q^{i} ; q_{\mu}^{i}\right),\left(x^{\mu}, q^{i}, r^{m} ; r_{\mu}^{m}, r_{i}^{m}\right),\left(x^{\mu}, q^{i}, r^{m} ; \tilde{q}_{\mu}^{i}, \tilde{r}_{\mu}^{m}\right) .
$$

Para os fibrados $\rho^{*} J E$ e $\rho^{*} J E \times_{F} J_{E} F$ sobre $F$, temos as coordenadas locais

$$
\left(x^{\mu}, q^{i}, r^{m} ; q_{\mu}^{i}\right),\left(x^{\mu}, q^{i}, r^{m} ; q_{\mu}^{i}, r_{\mu}^{m}, r_{i}^{m}\right) .
$$

Lema 2.1 [35] Existe um homomorfismo estrito de fibrados afins sobre $F$

$$
\gamma: \rho^{*} J E \times_{F} J_{E} F \longrightarrow J_{M} F
$$

que, em coordenadas locais, é dado por

$$
\tilde{q}_{\mu}^{i}=q_{\mu}^{i}, \quad \tilde{r}_{\mu}^{m}=r_{\mu}^{m}+r_{i}^{m} q_{\mu}^{i} .
$$

Passando à discussão de conexões, temos nestas mesmas coordenadas locais,

$$
\begin{gathered}
\Gamma_{E}=d x^{\mu} \otimes\left(\partial_{\mu}+\Gamma_{\mu}^{i} \partial_{i}\right) \\
\hat{\Gamma}_{F}=d x^{\mu} \otimes\left(\partial_{\mu}+\hat{\Gamma}_{\mu}^{i} \partial_{i}+\hat{\Gamma}_{\mu}^{m} \partial_{m}\right) \\
\tilde{\Gamma}_{F}=d x^{\mu} \otimes\left(\partial_{\mu}+\tilde{\Gamma}_{\mu}^{m} \partial_{m}\right)+d q^{i} \otimes\left(\partial_{i}+\tilde{\Gamma}_{i}^{m} \partial_{m}\right)
\end{gathered}
$$

Dizemos que $\hat{\Gamma}_{F}$ é projetável e projeta sobre $\Gamma_{E}$ se o diagrama

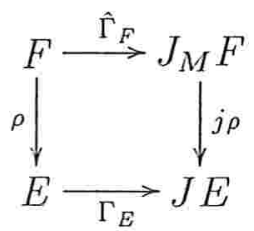


for comutativo. É possível observar que, em coordenadas locais, esta condição é equivalente a requerer que

$$
\hat{\Gamma}_{\mu}^{i}=\Gamma_{\mu}^{i}
$$

Dizemos ainda que $\tilde{\Gamma}_{F}$ e $\Gamma_{E}$ induzem $\hat{\Gamma}_{F}$ se o diagrama

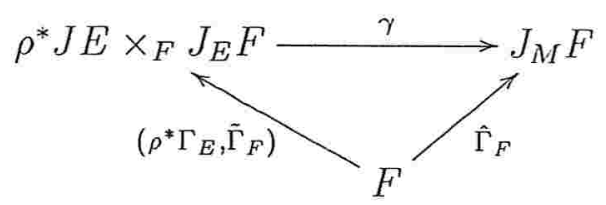

for comutativo, isto é, se

$$
\hat{\Gamma}_{F}=\gamma \circ\left(\rho^{*} \Gamma_{E}, \tilde{\Gamma}_{F}\right) .
$$

Esta conexão é chamada a conexão composta. Em coordenadas locais,

$$
\hat{\Gamma}_{F}=d x^{\mu} \otimes\left(\partial_{\mu}+\Gamma_{\mu}^{i} \partial_{i}+\left(\tilde{\Gamma}_{\mu}^{m}+\tilde{\Gamma}_{i}^{m} \Gamma_{\mu}^{i}\right) \partial_{m}\right)
$$

Usando o conceito de conexões parciais introduzido na seção anterior, podemos dar uma interpretação alternativa e bastante intuitiva a esta construção, baseada na decomposição (2.6) do fibrado tangente $T E$ de $E$ na soma direta do fibrado vertical $V E$ com o fibrado horizontal $H E$ fornecido pela conexão $\Gamma_{E}$. De fato, esta permite decompor qualquer conexão geral $\tilde{\Gamma}_{F}$ em $F \stackrel{\rho}{\longrightarrow} E$ na soma direta de duas partes: uma "parte vertical" $\tilde{\Gamma}_{F}^{V}$ e uma "parte horizontal" $\tilde{\Gamma}_{F}^{H}$ que são, respectivamente, conexões parciais gerais em $F \stackrel{\rho}{\longrightarrow} E$ ao longo de $V E$ e ao longo de $H E$. Observa-se então que se $\tilde{\Gamma}_{F}$ for projetável sobre $\Gamma_{E}$, então $\tilde{\Gamma}_{F}^{H}$ será, grosso modo, o "pull-back" da conexão composta $\hat{\Gamma}_{F}$ pela projeção $\pi$ de $E$ sobre $M$.

Para melhor entender essa última correspondência, vamos primeiro explicar com um pouco mais de detalhe o significado da definição dada na seção anterior para os casos da "parte vertical" $\tilde{\Gamma}_{F}^{V}$ e da "parte horizontal" $\tilde{\Gamma}_{F}^{H}$ da conexão $\tilde{\Gamma}_{F}$. Em ambos os casos, precisamos substituir, nas equações (2.62)-(2.65), $E$ por $F, M$ por $E, \pi$ por $\rho$ e $V E$ por $V_{E} F$. No primeiro caso, onde $D=V E$, observamos que o subfibrado vetorial $T_{V E} F$ de $T F$ é exatamente o fibrado vertical $V_{M} F$ em relação à projeção composta de $F$ sobre $M$ :

$$
T_{V E} F:=(T \rho)^{-1}(V E)=V_{M} F .
$$

Assim, a conexão parcial $\tilde{\Gamma}_{F}^{V}$ é dada pela escolha de um fibrado horizontal parcial $H_{V E} F$ tal que

$$
V_{M} F=H_{V E} F \oplus V_{E} F
$$

e que é a imagem de $\rho^{*} V E$ sob uma cisão

$$
\rho^{*} V E \longrightarrow V_{M} F
$$


da seguinte sequência exata de fibrados vetoriais sobre $F$ :

$$
0 \longrightarrow V_{E} F \longrightarrow V_{M} F \longrightarrow \rho^{*} V E \longrightarrow 0
$$

No segundo caso, o subfibrado vetorial $T_{H E} F$ de $T F$ não possui nenhuma interpretação alternativa particularmente interessante; temos apenas a definição:

$$
T_{H E} F:=(T \rho)^{-1}(H E) \text {. }
$$

Assim, a conexão parcial $\tilde{\Gamma}_{F}^{H}$ é dada pela escolha de um fibrado horizontal parcial $H_{H E} F$ tal que

$$
T_{H E} F=H_{H E} F \oplus V_{E} F
$$

e que é a imagem de $\rho^{*} H E$ sob uma cisão

$$
\rho^{*} H E \longrightarrow T_{H E} F
$$

da seguinte sequência exata de fibrados vetoriais sobre $F$ :

$$
0 \longrightarrow V_{E} F \longrightarrow T_{H E} F \longrightarrow \rho^{*} H E \longrightarrow 0 .
$$

Em termos de jatos, $\tilde{\Gamma}_{F}^{V}$ pode ser descrita como uma seção global

$$
\tilde{\Gamma}_{F}^{V}: F \longrightarrow J_{E}^{V} F
$$

onde $J_{E}^{V} F$ é o fibrado sobre $F$ cuja fibra em cada ponto $f \in F$ sobre o ponto $e \in E$ é o espaço afim

$$
\left(J_{E}^{V}\right)_{f} F=\left\{u \in L\left(V_{e} E, T_{f} F\right) / T_{f} \rho \circ u=\mathrm{id}_{V_{e} E}\right\}
$$

que é um subespaço afim do espaço vetorial $L\left(V_{e} E, T_{f} F\right)$, com espaço modelo $L\left(V_{e} E, V_{f} F\right)$. Deixando $f$ variar sobre $F$, obtemos um fibrado afim sobre $F$ em relação à projeção alvo $\tau_{F}^{V}: J_{E}^{V} F \rightarrow F$, que é um subfibrado do fibrado vetorial $L\left(\rho^{*} V E, T F\right) \cong \rho^{*}\left(V^{*} E\right) \otimes T F$. De forma semelhante, $\tilde{\Gamma}_{F}^{H}$ pode ser descrita como uma seção global

$$
\tilde{\Gamma}_{F}^{H}: F \longrightarrow J_{E}^{H} F
$$

onde $J_{E}^{H} F$ é o fibrado sobre $F$ cuja fibra em cada ponto $f \in F$ sobre o ponto $e \in E$ é o espaço afim

$$
\left(J_{E}^{H}\right)_{f} F=\left\{u \in L\left(H_{e} E, T_{f} F\right) / T_{f} \rho \circ u=\operatorname{id}_{H_{e} E}\right\},
$$

que é um subespaço afim do espaço vetorial $L\left(H_{e} E, T_{f} F\right)$, com espaço modelo $L\left(H_{e} E, V_{f} F\right)$. Deixando $f$ variar sobre $F$, obtemos um fibrado afim sobre $F$ em relação à projeção alvo $\tau_{F}^{H}: J_{E}^{H} F \rightarrow F$, que é um subfibrado do fibrado vetorial $L\left(\rho^{*} H E, T F\right) \cong \rho^{*}\left(H^{*} E\right) \otimes T F$. 
Por outro lado, sabemos que $J_{M} F$ é o fibrado sobre $F$ cuja fibra em cada ponto $f \in F$ sobre o ponto $e \in E$ que por sua vez está sobre o ponto $x \in M$ é o espaço afim

$$
\left(J_{M}\right)_{f} F=\left\{u \in L\left(T_{x} M, T_{f} F\right) / T_{f}(\rho \circ \pi) \circ u=\mathrm{id}_{T_{x} M}\right\} .
$$

Assim, o isomorfismo linear $T_{e} \pi$ entre $H_{e} E$ e $T_{x} M$ induz um isomorfismo afim natural entre $\left(J_{E}^{H}\right)_{f} F$ e $\left(J_{M}\right)_{f} F$, mostrando como identificar seções $\tilde{\Gamma}_{F}^{H}$ de $J_{E}^{H} F$ e seções $\hat{\Gamma}_{F}$ de $J_{M} F$ sobre $F$.

Na discussão a seguir, tornar-se-á importante que estruturas adicionais sobre $F$ como fibrado sobre $E$ sejam parcialmente herdadas não apenas pelo fibrado $J_{E} F$ mas também pelo fibrado $J_{M} F$. Em particular, se $F$ for um fibrado vetorial/afim sobre $E$, então como já foi visto na seção (1.3), $J_{E} F$ também será um fibrado vetorial/afim sobre $E$, mas podemos além disso afirmar que $J_{M} F$ será um fibrado vetorial/afim sobre $J E$. A demonstração desta afirmação está baseada na seguinte observação:

- Para quaisquer duas seções $\varphi$ e $\psi$ de $F$ sobre $M$ tais que em um ponto $x$ de $M$ dado, vale $j_{x}(\rho \circ \varphi)=j_{x}(\rho \circ \psi)$ em $J E$ (o que implica que $(\rho \circ \varphi)(x)=(\rho \circ \psi)(x)$ em E), é possível encontrar outras duas seções $\tilde{\varphi}$ e $\tilde{\psi}$ de $F$ sobre $M$ tais que $j_{x} \tilde{\varphi}=j_{x} \varphi$ e $j_{x} \tilde{\psi}=j_{x} \psi$ em $J_{M} F$ e que satisfazem $\rho \circ \tilde{\varphi}=\rho \circ \tilde{\psi}$ sobre $M$ inteiro.

De fato, usando esta observação, torna-se possível definir a estrutura linear/afim de $J_{M} F$ sobre $J E$ induzida pela estrutura linear/afim de $F$ sobre $E$ da mesma forma como a de $J_{E} F$ sobre $E$, por uma simples adaptação das fórmulas (1.20) e (1.21): No caso linear, podemos definir

$$
\lambda j_{x} \varphi+\mu j_{x} \psi=j_{x}(\lambda \varphi+\mu \psi)
$$

para $\lambda, \mu \in \mathbb{R}$ e $\varphi, \psi \in \Gamma(M, F)$ tais que $\rho \circ \varphi=\rho \circ \psi$ sobre $M$, enquanto que no caso afim, podemos definir

$$
j_{x} \varphi+j_{x} \vec{\varphi}=j_{x}(\varphi+\vec{\varphi})
$$

para $\varphi \in \Gamma(M, F)$ e $\vec{\varphi} \in \Gamma(M, \vec{F})$ tais que $\rho \circ \varphi=\rho \circ \vec{\varphi}$ sobre $M$. Essa estrutura nos permite introduzir a seguinte terminologia.

Definição 2.6 Seja $F$ um fibrado vetorial/afim sobre $E$ que por sua vez é um fibrado geral sobre $M$. Dada uma conexão geral $\Gamma_{E}$ em $E$ e uma conexão $\hat{\Gamma}_{F}$ em $F$ como fibrado composto sobre $M$ que é projetável e projeta sobre $\Gamma_{E}$, dizemos que a conexão $\hat{\Gamma}_{F}$ é linear/afim ao longo das fibras de $F$ sobre $E$ se e somente se o diagrama (2.84) definir um homomorfismo de fibrados vetoriais/afins (sobre $\Gamma_{E}$ ).

Em termos das componentes utilizadas na equação (2.82), isso significa que em coordenadas locais lineares/afins ao longo das fibras de $F$ sobre $E$,

$$
\hat{\Gamma}_{\mu}^{m}(x, q, r)=-A_{\mu n}^{m}(x, q) r^{n},
$$


no caso linear, e

$$
\hat{\Gamma}_{\mu}^{m}(x, q, r)=-A_{\mu n}^{m}(x, q) r^{n}-A_{\mu}^{m}(x, q),
$$

no caso afim.

Voltando à situação geral, vimos que fixando um fibrado composto $F \stackrel{\rho}{\longrightarrow} E \stackrel{\pi}{\longrightarrow} M$ e uma conexão $\Gamma_{E}$ em $E \stackrel{\pi}{\longrightarrow} M$, uma conexão $\tilde{\Gamma}_{F}$ em $F \stackrel{\rho}{\longrightarrow} E$ induz uma conexão $\hat{\Gamma}_{F}$ em $F \stackrel{\pi^{\circ} \rho}{\longrightarrow} M$, a conexão composta, que é obviamente projetável e projeta sobre $\Gamma_{E}$. Reciprocamente, podemos perguntar sob quais condições é possível, para uma dada conexão $\hat{\Gamma}_{F}$ em $F \stackrel{\pi^{\circ} \rho}{\longrightarrow} M$ que é projetável e projeta sobre $\Gamma_{E}$, construir uma conexão $\tilde{\Gamma}_{F}$ em $F \stackrel{\rho}{\longrightarrow} E$ tal que $\hat{\Gamma}_{F}$ seja a composta de $\tilde{\Gamma}_{F}$ com $\Gamma_{E}$, ou seja,

$$
\hat{\Gamma}_{\mu}^{m}=\tilde{\Gamma}_{\mu}^{m}+\tilde{\Gamma}_{i}^{m} \Gamma_{\mu}^{i},
$$

e tal que $\tilde{\Gamma}_{F}$ seja determinada de forma natural a partir de $\hat{\Gamma}_{F}$ e $\Gamma_{E}$. É claro que a condição (2.100) por si só não determina $\tilde{\Gamma}_{F}$ unicamente e portanto uma construção "natural" de $\tilde{\Gamma}_{F}$ em termos de $\hat{\Gamma}_{F}$ e $\Gamma_{E}$ requer dados adicionais. É de se esperar que tal construção "natural" seja possível se o fibrado composto $F \stackrel{\pi \circ \rho}{\longrightarrow} M$ for obtido a partir do fibrado original $E \stackrel{\pi}{\longrightarrow} M$ por alguma construção funtorial "fibra por fibra" que, via de regra, também permite obter a conexão $\hat{\Gamma}_{F}$ a partir da conexão $\Gamma_{E}$. Exemplos bem conhecidos de tais construções para fibrados vetoriais e conexões lineares são o dual, a soma direta e o produto tensorial [19]; uma formulação próxima à notação aqui empregada pode ser encontrada em [28, pg 52]. ${ }^{1}$ Exemplos importantes para fibrados e conexões gerais são o fibrado vertical e o fibrado dos jatos de primeira ordem; a correspondente construção de $\hat{\Gamma}_{F}$ a partir de $\Gamma_{E}$ é descrita em [28, pgs 53 \& 57] sob o título "vertical connection" e "second order connection", respectivamente. No entanto, a questão da construção de $\tilde{\Gamma}_{F}$ não é abordada: ela sempre requer dados adicionais.

A seguir, discutiremos a construção de conexões em vários fibrados construídos a partir de um fibrado geral $E \stackrel{\pi}{\longrightarrow} M$ que são fibrados vetoriais ou afins sobre $E$, incluindo o fibrado vertical $V E$ de $E$, o fibrado dos jatos de primeira ordem $J E$ de $E$ e o fibrado dos cojatos $J{ }^{\circledast} E$ de $E$ : esta última será a chave para o entendimento global da construção do tensor de Poisson a ser apresentada na seção final deste capítulo.

\subsection{Conexões no fibrado vertical}

Nesta seção, discutimos a construção de conexões em $V E$, o fibrado vertical de $E$, primeiro como fibrado composto sobre $M$

$$
V E \stackrel{\tau_{E}}{\longrightarrow} E \stackrel{\pi}{\longrightarrow} M
$$

\footnotetext{
${ }^{1}$ Uma formulação axiomática do que deve ser entendido por uma construção funtorial "fibra por fibra", no caso de fibrados vetoriais, pode ser encontrada no livro [27].
} 
e a seguir como fibrado vetorial sobre $E$. A primeira parte da construção pode ser elaborada de maneira canônica, enquanto que a segunda depende da escolha de uma conexão parcial linear em $V E$ ao longo do próprio $V E$.

Inicialmente, recordamos que se $E$ e $E^{\prime}$ são fibrados sobre $M$ e $\phi: E \rightarrow E^{\prime}$ é um homomorfismo de fibrados sobre $M$, então a aplicação tangente $T \phi: T E \rightarrow T E^{\prime}$ levará o fibrado vertical $V E$ de $E$ para o fibrado vertical $V E^{\prime}$ de $E^{\prime}$ e portanto induz uma aplicação vertical tangente $V \phi: V E \rightarrow V E^{\prime}$, por restrição. Aplicamos esta observação a duas situações. Primeiro, substituindo $E$ por $J E, E^{\prime}$ por $E$ e $\phi$ pela projeção alvo $\tau_{E}$, obtemos uma projeção $V \tau_{E}: V_{M}(J E) \rightarrow V E$. Segundo, mantendo $E$ mas substituindo $E^{\prime}$ por $J E$ e $\phi$ por uma aplicação $\Gamma_{E}: E \rightarrow J E$, obtemos uma aplicação $V \Gamma_{E}: V E \rightarrow V_{M}(J E)$. Quando $\Gamma_{E}$ for uma conexão geral em $E$, temos $\tau_{E} \circ \Gamma_{E}=\mathrm{id}_{E}$ e, pela regra da cadeia, $V \tau_{E} \circ V \Gamma_{E}=\mathrm{id} V E$. Agora fazemos uso da seguinte

Proposição 2.1 Existe um difeomorfismo global entre $V_{M}(J E)$ e $J_{M}(V E)$, que também é um isomorfismo global de fibrados sobre $E$ assim como sobre $M$, tal que o seguinte diagrama comuta:

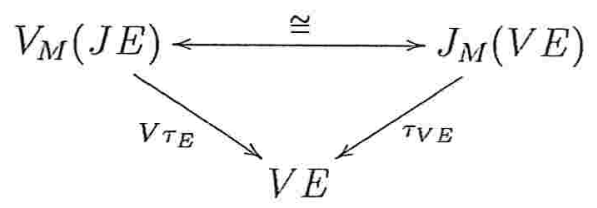

Em coordenadas locais, ele é dado pelo seguinte operador de troca:

$$
\begin{array}{ccc}
V_{M}(J E) & \longrightarrow & J_{M}(V E) \\
\left(x^{\mu}, q^{i}, q_{\mu}^{i}, \dot{q}^{i}, \dot{q}_{\mu}^{i}\right) & \longmapsto & \left(x^{\mu}, q^{i}, \dot{q}^{i}, q_{\mu}^{i}, \dot{q}_{\mu}^{i}\right)
\end{array}
$$

DEMONSTRAÇÃo. Para encontrar uma formulação global da definição do operador de troca dado, em coordenadas locais, pela equação (2.103), começamos com uma convenção: uma família a um parâmetro de seções de $E$ é simplesmente a segunda componente $\varphi$ de um seção $\tilde{\varphi}$ de $I \times E$ sobre $I \times M$, onde $I \subset \mathbb{R}$ é algum intervalo contendo 0 :

$$
\tilde{\varphi}(t, x)=(t, \varphi(t, x)) \text {. }
$$

Neste caso, escrevemos

$$
\varphi_{t}(x)=\varphi(t, x), \dot{\varphi}_{0}(x)=\left.\frac{d}{d t} \varphi_{t}(x)\right|_{t=0} .
$$

A seguir, fixamos pontos de referência $m \in M$ e $e \in E$ sobre $m$, um vetor vertical $v \in V_{e} E$ e um jato $u \in J_{e} E$. 
1. Seja $w_{1} \in V_{M}(J E)$ um vetor vertical tangente ao fibrado $J E \longrightarrow M$ sobre $u$. Usando coordenadas locais, pode-se mostrar que existe uma família a um parâmetro de seções de $E$ tal que $\varphi_{0}(m)=e, \pi\left(\varphi_{t}(m)\right)=m$ para todo $t \in I, \dot{\varphi}_{0}(m)=v, j_{m} \varphi_{0}=u \mathrm{e}$ $\left.\frac{d}{d t} j_{m} \varphi_{t}\right|_{t=0}=w_{1}$. Então $\dot{\varphi}_{0}$ é uma seção de $V E \longrightarrow M$, e definimos $w_{2} \in J_{M}(V E)$ por $w_{2}=j_{m} \dot{\varphi}_{0}$. Como veremos, $w_{2}$ não depende da escolha de $\varphi$, desde que suponhamos que $\varphi$ seja pelo menos de classe $C^{2}$, para garantir a validade da regra de Schwarz.

2. Seja $w_{2} \in J_{M}(V E)$ um jato de primeira ordem do fibrado $V E \longrightarrow M$ sobre $v$. Usando coordenadas locais, pode-se mostrar que existe uma família a um parâmetro de seções de $E$ tal que $\varphi_{0}(m)=e, \pi\left(\varphi_{t}(m)\right)=m$ para todo $t \in I, \dot{\varphi}_{0}(m)=v, j_{m} \varphi_{0}=u$ e $j_{m} \dot{\varphi}_{0}=w_{2}$. Então a função $I \rightarrow J E$ definida por $t \mapsto j_{m} \varphi_{t}$ é uma curva em $J E$ que é vertical em relação à projeção fonte, e definimos $w_{1} \in V_{M}(J E)$ por $w_{1}=\left.\frac{d}{d t} j_{m} \varphi_{t}\right|_{t=0}$. Como veremos, $w_{1}$ não depende da escolha de $\varphi$, desde que suponhamos que $\varphi$ seja pelo menos de classe $C^{2}$, para garantir a validade da regra de Schwarz.

Em coordenadas locais, as condições impostas sobre a família a um parâmetro de seções de $E$ utilizada fixam o valor de $\varphi$, de suas primeiras derivadas parciais e de suas segundas derivadas parciais mistas no ponto $(0, m)$ :

- $\varphi(0, m)$ corresponde a $e$;

- $\frac{\partial \varphi}{\partial t}(0, m)$ corresponde a $v$ e $\frac{\partial \varphi}{\partial x^{\mu}}(0, m)$ corresponde a $u$;

- $\frac{\partial^{2} \varphi}{\partial t \partial x^{\mu}}(0, m)$ corresponde a $w_{1}$ e $\frac{\partial^{2} \varphi}{\partial x^{\mu} \partial t}(0, m)$ corresponde a $w_{2}$.

Portanto, a identificação entre $w_{1}$ e $w_{2}$ é expressão da regra de Schwarz.

Assim, dada uma conexão geral

$$
\begin{array}{cccc}
\Gamma_{E}: & E & \longrightarrow & J E \\
\left(x^{\mu}, q^{i}\right) & \longmapsto & \left(x^{\mu}, q^{i}, \Gamma_{\mu}^{i}\right)
\end{array}
$$

podemos formar sua aplicação vertical tangente

$$
\begin{array}{cccc}
V \Gamma_{E}: & V E & \longrightarrow & V_{M}(J E) \\
& \left(x^{\mu}, q^{i}, \dot{q}^{i}\right) & \longmapsto & \left(x^{\mu}, q^{i}, \Gamma_{\mu}^{i}, \dot{q}^{i}, \partial_{j} \Gamma_{\mu}^{i} \dot{q}^{j}\right)
\end{array}
$$

e compô-la com o isomorfismo da Proposição 2.1 para obter uma aplicação

$$
\hat{\Gamma}_{V E}: V E \longrightarrow J_{M}(V E)
$$


que satisfaz $\tau_{V E} \circ \hat{\Gamma}_{V E}=\mathrm{id}_{V E}$ e portanto é uma conexão geral em $V E$; ela é chamada a conexão vertical induzida por $\Gamma_{E}$. Em coordenadas locais, ela é dada por

$$
\hat{\Gamma}_{V E}\left(\partial_{\mu}\right)=\partial_{\mu}+\Gamma_{\mu}^{i} \partial_{i}+\partial_{j} \Gamma_{\mu}^{k} \dot{q}^{j} \dot{\partial}_{k}
$$

ou equivalentemente

$$
\hat{\Gamma}_{V E}=d x^{\mu} \otimes\left(\partial_{\mu}+\Gamma_{\mu}^{i} \partial_{i}+\partial_{j} \Gamma_{\mu}^{k} \dot{q}^{j} \dot{\partial}_{k}\right)
$$

Obviamente, $\hat{\Gamma}_{V E}$ é projetável e projeta sobre $\Gamma_{E}$.

Passando a considerar $V E$ não como fibrado sobre $M$ e sim como fibrado vetorial sobre $E$, e conforme foi visto na seção anterior, podemos reinterpretar a conexão vertical $\hat{\Gamma}_{V E}$ assim definida como uma conexão parcial $\tilde{\Gamma}_{V E}^{H}$ em $V E$ ao longo do fibrado horizontal $H E$ definido por $\Gamma_{E}$. Ademais, a linearidade de $\hat{\Gamma}_{V E}$ ao longo das fibras de $V E$ implica que $\tilde{\Gamma}_{V E}^{H}$ será uma conexão linear parcial ao longo de $H E$. Complementando esta por $\tilde{\Gamma}_{V E}^{V}$, uma conexão linear parcial qualquer ao longo de $V E$, chegamos à conclusão que é sempre possível reinterpretar $\hat{\Gamma}_{V E}$ como sendo o resultado da composição de alguma conexão linear $\tilde{\Gamma}_{V E}$ em $V E$ com a conexão original $\Gamma_{E}$. Em coordenadas locais, esta é dada por

$$
\begin{aligned}
\tilde{\Gamma}_{V E}\left(\partial_{\mu}+\Gamma_{\mu}^{i} \partial_{i}\right) & =\partial_{\mu}+\Gamma_{\mu}^{i} \partial_{i}+\partial_{j} \Gamma_{\mu}^{k} \dot{q}^{j} \dot{\partial}_{k}, \\
\tilde{\Gamma}_{V E}\left(\partial_{i}\right) & =\partial_{i}-\Gamma_{i j}^{k} \dot{q}^{j} \dot{\partial}_{k} .
\end{aligned}
$$

ou equivalentemente

$$
\tilde{\Gamma}_{V E}=d x^{\mu} \otimes\left(\partial_{\mu}+\Gamma_{\mu}^{i} \partial_{i}+\partial_{j} \Gamma_{\mu}^{k} \dot{q}^{j} \dot{\partial}_{k}\right)+\left(d q^{i}-\Gamma_{\mu}^{i} d x^{\mu}\right) \otimes\left(\partial_{i}-\Gamma_{i j}^{k} \dot{q}^{j} \dot{\partial}_{k}\right),
$$

o que pode ser reescrito na forma

$$
\tilde{\Gamma}_{V E}=d x^{\mu} \otimes\left(\partial_{\mu}+\left(\partial_{j} \Gamma_{\mu}^{k}+\Gamma_{\mu}^{i} \Gamma_{i j}^{k}\right) \dot{q}^{j} \dot{\partial}_{k}\right)+d q^{i} \otimes\left(\partial_{i}-\Gamma_{i j}^{k} \dot{q}^{j} \dot{\partial}_{k}\right)
$$

Observa-se que os coeficientes adicionais $\Gamma_{i j}^{k}$ introduzidos por estas fórmulas são os símbolos de Christoffel da conexão linear parcial $\tilde{\Gamma}_{V E}^{V}$. Resumindo, temos

Teorema 2.1 Dadas uma conexão $\Gamma_{E}$ em $E$ sobre $M$ e uma conexão linear parcial $\tilde{\Gamma}_{V E}^{V E}$ em $V E$ sobre $E$ ao longo de $V E$, existe uma única extensão de $\tilde{\Gamma}_{V E}^{V E}$ para uma conexão linear total $\tilde{\Gamma}_{V E}$ em $V E$ sobre $E$ cuja composição com $\Gamma_{E}$ é a conexão vertical $\hat{\Gamma}_{V E}$ em VE sobre $M$ induzida por $\Gamma_{E}$. Em termos de derivadas covariantes de seçóes $X$ de $V E$, isto é, campos vetoriais verticais $X$ sobre $E$, temos

$$
\nabla_{i} X^{j}=\partial_{i} X^{j}+\Gamma_{i r}^{j} X^{r}
$$

$e$

$$
\nabla_{\mu} X^{j}=\partial_{\mu} X^{j}-\left(\partial_{i} \Gamma_{\mu}^{j}+\Gamma_{r i}^{j} \Gamma_{\mu}^{r}\right) X^{i}
$$


ou, equivalentemente,

$$
\nabla_{\Gamma\left(\partial_{\mu}\right)} X^{j}=\partial_{\mu} X^{j}+\Gamma_{\mu}^{i} \partial_{i} X^{j}-\partial_{i} \Gamma_{\mu}^{j} X^{i}
$$

Uma demonstração independente de que estas expressões realmente definem uma derivada covariante de seções $X$ de $V E$ encontra-se na primeira seção do Apêndice. Neste contexto, é interessante observar que conforme a equação (A.21), os campos de coordenadas $\partial_{i}$ sobre $E$ se transformam de maneira covariante, enquanto que os campos de coordenadas $\partial_{\mu}$ sobre $E$ não. No entanto, combinando as equações (A.19), (A.21) e (2.14), concluímos que os levantamentos horizontais dos campos de coordenadas $\partial_{\mu}$ sobre $M$ para $E$, estes sim, se transformam de maneira covariante.

Finalmente, observamos que se o fibrado $E$ for vetorial ou afim, então podemos supor sem perda de generalidade que a conexão $\tilde{\Gamma}_{V E}^{V E}$ tenha curvatura zero. Em coordenadas locais que são, respectivamente, lineares ou afins ao longo das fibras de $E$, podemos neste caso supor que $\Gamma_{i r}^{j}=0$.

\subsection{Conexões nos fibrados de jatos e cojatos}

Nesta seção, discutimos a construção de conexões no fibrado $J E$ dos jatos de primeira ordem de $E$ e no fibrado $J^{\otimes} E$ dos cojatos estendido de $E$, diretamente como fibrado afim e como fibrado vetorial sobre $E$, respectivamente. Como ponto de partida, teremos que escolher: (a) uma conexão $\Gamma_{E}$ em $E$ sobre $M$, (b) uma conexão linear parcial $\tilde{\Gamma}_{V E}^{V E}$ em $V E$ sobre $E$ ao longo de $V E$ e (c) uma conexão linear $\Gamma_{M}$ na variedade base $M$ do fibrado original.

No primeiro passo, observamos que o fibrado de diferenças de $J E$ sobre $E$ é o fibrado vetorial

$$
\vec{J} E \cong \pi^{*}\left(T^{*} M\right) \otimes V E .
$$

Portanto, podemos fazer uso da conexão linear $\Gamma_{V E}$ em $V E$ sobre $E$ induzida pelas primeiras duas conexões, conforme o Teorema 2.1 da seção anterior (o símbolo ? será omitido para simplificar a notação), em conjunto com a terceira conexão (ou mais exatamente seu pullback de $M$ para $E$ ), para definir uma conexão linear $\Gamma_{\vec{J} E}$, como produto tensorial

$$
\Gamma_{\vec{J} E}=\Gamma_{M}^{*} \otimes \Gamma_{V E} .
$$

No segundo passo, aplicamos um resultado enunciado na Seção 2.3, segundo o qual existe uma única conexão afim $\Gamma_{J E}$ em $J E$ que tenha $\Gamma_{\vec{J} E}$ como parte linear e tal que a conexão $\Gamma_{E}$, vista como seção de $J E$ sobre $E$, seja covariantemente constante:

$$
\nabla^{\Gamma_{J E}} \Gamma_{E}=0
$$


Em termos de coordenadas locais $\left(x^{\mu}, q^{i} ; \vec{q}_{\mu}^{i}\right)$ para $\vec{J} E$ e $\left(x^{\mu}, q^{i} ; q_{\mu}^{i}\right)$ para $J E$, usamos que os símbolos de Christoffel do produto tensorial de duas conexões lineares são obtidos somando os símbolos de Christoffel dos dois fatores, em conjunto com a equação (2.112), digamos, para obter

$$
\begin{aligned}
\Gamma_{\vec{J} E}= & d x^{\mu} \otimes\left(\partial_{\mu}+\left(\left(\partial_{j} \Gamma_{\mu}^{k}+\Gamma_{\mu}^{i} \Gamma_{i j}^{k}\right) \vec{q}_{\kappa}^{j}+\Gamma_{\mu \kappa}^{\rho} \vec{q}_{\rho}^{k}\right) \partial_{k}^{\kappa}\right) \\
& +d q^{i} \otimes\left(\partial_{i}-\Gamma_{i j}^{k} \vec{q}_{\kappa}^{j} \partial_{k}^{\kappa}\right),
\end{aligned}
$$

e combinamos as equações $(2.37),(2.47),(2.51)$ e (2.53) para concluir que

$$
\begin{aligned}
\Gamma_{J E}=d x^{\mu} \otimes\left(\partial_{\mu}+\right. & \left(\left(\partial_{j} \Gamma_{\mu}^{k}+\Gamma_{\mu}^{i} \Gamma_{i j}^{k}\right) q_{\kappa}^{j}+\Gamma_{\mu \kappa}^{\rho} q_{\rho}^{k}\right) \partial_{k}^{\kappa} \\
& \left.+\left(\partial_{\mu} \Gamma_{\kappa}^{k}-\left(\partial_{j} \Gamma_{\mu}^{k}+\Gamma_{\mu}^{i} \Gamma_{i j}^{k}\right) \Gamma_{\kappa}^{j}-\Gamma_{\mu \kappa}^{\rho} \Gamma_{\rho}^{k}\right) \partial_{k}^{\kappa}\right) \\
+d q^{i} \otimes\left(\partial_{i}-\right. & \left.\Gamma_{i j}^{k} q_{\kappa}^{j} \partial_{k}^{\kappa}+\left(\partial_{i} \Gamma_{\kappa}^{k}+\Gamma_{i j}^{k} \Gamma_{\kappa}^{j}\right) \partial_{k}^{\kappa}\right) .
\end{aligned}
$$

Passando aos duais, usamos a equação (2.36) para definir a conexão linear $\Gamma_{\vec{J}^{*} E}$ em $\vec{J}^{*} E$, dual à conexão linear $\Gamma_{\vec{J} E}$ em $\overrightarrow{J E}$, e da mesma maneira, usamos a equação (2.59) para definir a conexão linear $\Gamma_{J^{\star} E}$ em $J^{\star} E$, dual à conexão afim $\Gamma_{J E}$ em $J E$. Finalmente, para incorporar os duais torcidos, temos que formar os produtos tensoriais das conexões lineares nos duais ordinários com a conexão linear $\Gamma_{M}^{* n}$ no fibrado em linhas $\bigwedge^{n} T^{*} M$ sobre $M$ induzida pela conexão linear $\Gamma_{M}^{*}$ em $T^{*} M$ :

$$
\begin{aligned}
& \Gamma_{\vec{J}^{\oplus} E}=\Gamma_{\vec{J}^{*} E} \otimes \Gamma_{M}^{* n}, \\
& \Gamma_{J^{\oplus} E}=\Gamma_{J^{\star} E} \otimes \Gamma_{M}^{* n} .
\end{aligned}
$$

Em termos de coordenadas locais $\left(x^{\mu}, q^{i} ; p_{i}^{\mu}\right)$ para $\vec{J}^{*} E$ e $\left(x^{\mu}, q^{i} ; p_{i}^{\mu}, p\right)$ para $J^{\star} E$, temos

$$
\begin{aligned}
\Gamma_{\vec{J}^{*} E}= & d x^{\mu} \otimes\left(\partial_{\mu}-\left(\left(\partial_{j} \Gamma_{\mu}^{k}+\Gamma_{\mu}^{i} \Gamma_{i j}^{k}\right) p_{k}^{\kappa}+\Gamma_{\mu \rho}^{\kappa} p_{j}^{\rho}\right) \partial_{\kappa}^{j}\right) \\
& +d q^{i} \otimes\left(\partial_{i}+\Gamma_{i j}^{k} p_{k}^{\kappa} \partial_{\kappa}^{j}\right),
\end{aligned}
$$

e

$$
\begin{aligned}
\Gamma_{J^{\star} E}= & d x^{\mu} \otimes\left(\partial_{\mu}-\left(\left(\partial_{j} \Gamma_{\mu}^{k}+\Gamma_{\mu}^{i} \Gamma_{i j}^{k}\right) p_{k}^{\kappa}+\Gamma_{\mu \rho}^{\kappa} p_{j}^{\rho}\right) \partial_{\kappa}^{j}\right. \\
& \left.-\left(\partial_{\mu} \Gamma_{\kappa}^{k}-\left(\partial_{j} \Gamma_{\mu}^{k}+\Gamma_{\mu}^{i} \Gamma_{i j}^{k}\right) \Gamma_{\kappa}^{j}-\Gamma_{\mu \kappa}^{\rho} \Gamma_{\rho}^{k}\right) p_{k}^{\kappa} \partial_{0}\right) \\
+d q^{i} \otimes & \left(\partial_{i}+\Gamma_{i j}^{k} p_{k}^{\kappa} \partial_{\kappa}^{j}-\left(\partial_{i} \Gamma_{\kappa}^{k}+\Gamma_{i j}^{k} \Gamma_{\kappa}^{j}\right) p_{k}^{\kappa} \partial_{0}\right) .
\end{aligned}
$$


De maneira análoga, em termos de coordenadas locais $\left(x^{\mu}, q^{i} ; p_{i}^{\mu}\right)$ para $\vec{J}^{\otimes} E$ e $\left(x^{\mu}, q^{i} ; p_{i}^{\mu}, p\right)$ para $J^{\circledast} E$, temos

$$
\begin{aligned}
\Gamma_{\vec{J}^{\oplus} E}= & d x^{\mu} \otimes\left(\partial_{\mu}-\left(\left(\partial_{j} \Gamma_{\mu}^{k}+\Gamma_{\mu}^{i} \Gamma_{i j}^{k}\right) p_{k}^{\kappa}+\Gamma_{\mu \rho}^{\kappa} p_{j}^{\rho}-\Gamma_{\mu \rho}^{\rho} p_{j}^{\kappa}\right) \partial_{\kappa}^{j}\right) \\
& +d q^{i} \otimes\left(\partial_{i}+\Gamma_{i j}^{k} p_{k}^{\kappa} \partial_{\kappa}^{j}\right),
\end{aligned}
$$

e

$$
\begin{aligned}
\Gamma_{J^{\oplus E}}=d x^{\mu} \otimes( & \partial_{\mu}-\left(\left(\partial_{j} \Gamma_{\mu}^{k}+\Gamma_{\mu}^{i} \Gamma_{i j}^{k}\right) p_{k}^{\kappa}+\Gamma_{\mu \rho}^{\kappa} p_{j}^{\rho}-\Gamma_{\mu \rho}^{\rho} p_{j}^{\kappa}\right) \partial_{\kappa}^{j} \\
& \left.-\left(\left(\partial_{\mu} \Gamma_{\kappa}^{k}-\left(\partial_{j} \Gamma_{\mu}^{k}+\Gamma_{\mu}^{i} \Gamma_{i j}^{k}\right) \Gamma_{\kappa}^{j}-\Gamma_{\mu \kappa}^{\rho} \Gamma_{\rho}^{k}\right) p_{k}^{\kappa}-\Gamma_{\mu \rho}^{\rho} p\right) \partial_{0}\right) \\
+d q^{i} \otimes & \left(\partial_{i}+\Gamma_{i j}^{k} p_{k}^{\kappa} \partial_{\kappa}^{j}-\left(\partial_{i} \Gamma_{\kappa}^{k}+\Gamma_{i j}^{k} \Gamma_{\kappa}^{j}\right) p_{k}^{\kappa} \partial_{0}\right) .
\end{aligned}
$$

Em resumo, temos

Teorema 2.2 Dadas uma conexão $\Gamma_{E}$ em $E$ sobre $M$, uma conexão linear parcial $\Gamma_{V E}^{V E}$ em $V E$ sobre $E$ ao longo de $V E$ e uma conexão linear $\Gamma_{M}$ em $M$, seja $\Gamma_{V E}$ a conexão linear no fibrado vertical $V E$, como fibrado vetorial sobre $E$, induzida pelas primeiras duas, conforme o Teorema 2.1. (O símbolo . será omitido, para simplificar a notação.) Então existem

- uma única conexão linear $\Gamma_{\vec{J} E}$ em $\overrightarrow{J E}$, como fibrado vetorial sobre $E$, que é o produto. tensorial de $\Gamma_{M}^{*}$ e $\Gamma_{V E}$ (veja a equação (2.117)), com expressão em coordenadas locais adaptadas dada pela equação (2.119);

- uma única conexão afim $\Gamma_{J E}$ em JE, como fibrado afim sobre $E$, que tem $\Gamma_{\overrightarrow{J E}}$ como parte linear e para a qual $\Gamma_{M}$ é uma seção covariantemente constante (veja a equação (2.118)), com expressão em coordenadas locais adaptadas dada pela equação (2.120);

- conexóes lineares $\Gamma_{\vec{J}^{*} E}$ em $\vec{J}^{*} E, \Gamma_{J^{\star} E}$ em $J^{\star} E, \Gamma_{\vec{J}^{\oplus E}}$ em $\vec{J}^{\circledast} E$ e $\Gamma_{J^{\oplus} E}$ em $J^{\oplus E}$, todos como fibrados vetoriais sobre $E$, unicamente determinadas por dualização e, no caso dos duais torcidos, mediante um produto tensorial com $\Gamma_{M}^{* n}$ (veja as equações (2.121) e (2.122)), com expressões em coordenadas locais adaptadas dada pelas equações (2.123)(2.126).

Finalmente, consideramos a questão de construir, a partir dos mesmos dados que antes, uma conexão afim no espaço $J{ }^{\circledast} E$ dos cojatos estendido de $E$ considerado como fibrado afim em linhas sobre o espaço $\vec{J}^{\otimes} E$ dos cojatos comum de $E$. No caso não-torcido, é fácil ver que a escolha de uma conexão $\Gamma_{E}$ em $E$ sobre $M$, vista como seção $\Gamma_{E}: E \rightarrow J E$, induz uma decomposição canônica de formas afins sobre as fibras de $J E$ em parte linear 
e parte constante, que pode ser vista como uma trivialização explícita de $J^{\star} E$ sobre $\vec{J}^{*} E$. Obviamente, esta induz uma conexão afim $\bar{\Gamma}_{J^{\star} E}$ em $J^{\star} E$ como fibrado afim em linhas sobre $\vec{J}^{*} E$. Tomando o produto tensorial com o fibrado em linhas $\bigwedge^{n} T^{*} M$ sobre $M$ e a conexão linear $\Gamma_{M}^{* n}$, obtemos uma conexão afim $\bar{\Gamma}_{J{ }^{\oplus E}}$ em $J^{\otimes} E$ como fibrado afim em linhas sobre $\vec{J}{ }^{\circledast} E$. Explicitamente, em termos de coordenadas locais $\left(x^{\mu}, q^{i} ; p_{i}^{\mu}\right)$ para $\vec{J}^{*} E$ e $\left(x^{\mu}, q^{i} ; p_{i}^{\mu}, p\right)$ para $J^{\star} E$, temos

$$
\begin{aligned}
\bar{\Gamma}_{J \star E}= & d x^{\mu} \otimes\left(\partial_{\mu}-\partial_{\mu} \Gamma_{\nu}^{j} p_{j}^{\nu} \partial_{0}\right) \\
& +d q^{i} \otimes\left(\partial_{i}-\partial_{i} \Gamma_{\nu}^{j} p_{j}^{\nu} \partial_{0}\right)+d p_{i}^{\mu} \otimes\left(\partial_{\mu}-\Gamma_{\mu}^{i} \partial_{0}\right) .
\end{aligned}
$$

De maneira análoga, em termos de coordenadas locais $\left(x^{\mu}, q^{i} ; p_{i}^{\mu}\right)$ para $\vec{J}^{\circledast} E$ e $\left(x^{\mu}, q^{i} ; p_{i}^{\mu}, p\right)$ para $J^{\ominus} E$, temos

$$
\begin{aligned}
\bar{\Gamma}_{J \Theta E}= & d x^{\mu} \otimes\left(\partial_{\mu}-\left(\partial_{\mu} \Gamma_{\nu}^{j} p_{j}^{\nu}-\Gamma_{\mu \rho}^{\rho}\left(p+\Gamma_{\nu}^{j} p_{j}^{\nu}\right)\right) \partial_{0}\right) \\
& +d q^{i} \otimes\left(\partial_{i}-\partial_{i} \Gamma_{\nu}^{j} p_{j}^{\nu} \partial_{0}\right)+d p_{i}^{\mu} \otimes\left(\partial_{\mu}-\Gamma_{\mu}^{i} \partial_{0}\right) .
\end{aligned}
$$

Veremos a seguir como estas construções são utilizadas para chegar à definição de um tensor de Poisson.

\subsection{Referenciais de Darboux e tensor de Poisson}

Inicialmente, lembremos que um referencial numa variedade $M$ de dimensão $n$ é uma base $\left\{e_{1}, \ldots, e_{n}\right\}$ de campos vetoriais locais $e_{\mu}$ sobre $M$. Tal referencial é chamado holônomo se estes estão em involução, i.e., se vale

$$
\left[e_{\mu}, e_{\nu}\right]=0 \quad(1 \leq \mu, \nu \leq n) .
$$

Neste caso, o teorema de Frobenius garante a existência de um sistema de coordenadas locais $x^{\mu}$ sobre $M$ tal que (após diminuição do domínio de definição se necessário),

$$
e_{\mu}=\frac{\partial}{\partial x^{\mu}} \quad(1 \leq \mu \leq n)
$$

Caso contrário, o referencial é dito não-holônomo.

No espaco total de um fibrado, existe uma classe de referenciais especiais adaptados a sua estrutura de fibrado: são os referenciais cujos membros podem naturalmente ser divididos em campos verticais e campos horizontais. Obviamente, tal divisão requer a escolha de uma 
conexão. Mais explicitamente, dado um fibrado com espaço total $E$, variedade base $M$ e fibra típica $Q$, podemos a partir de um sistema de coordenadas locais $x^{\mu}$ para $M$, um sistema de coordenadas locais $q^{i}$ para $Q$ e uma conexão $\Gamma$ construir um referencial em $E$ constituído de $N=\operatorname{dim} Q$ campos vetoriais locais verticais $e_{i}$ mais $n=\operatorname{dim} M$ campos vetoriais locais horizontais $e_{\mu}$, definidos por

$$
e_{i}=\frac{\partial}{\partial q^{i}}
$$

$\mathrm{e}$

$$
e_{\mu}=\frac{\partial}{\partial x^{\mu}}+\Gamma_{\mu}^{i} \frac{\partial}{\partial q^{i}}
$$

Em geral, este referencial composto não é holônomo, apesar dos referenciais em $M$ e em $Q$ formados, respectivamente, pelos $\partial / \partial x^{\mu}$ e pelos $\partial / \partial q^{i}$, por si só serem holônomos. A condição para o referencial composto ser holônomo é que a conexão $\Gamma$ tenha curvatura zero; neste caso, ele é associado a um sistema de coordenadas composto $\left(x^{\mu}, q^{i}\right)$, como usado anteriormente.

Usando esta idéia e os resultados da seção anterior, construiremos uma classe de referenciais no fibrado dos cojatos estendido $J^{\circledast} E$ que chamaremos de referenciais de Darboux.

Na geometria simplética, um sistema de coordenadas adaptado à estrutura simplética é chamado um sistema de coordenadas de Darboux. A mesma terminologia será adotada para a geometria multissimplética. Como exemplos, citamos as coordenadas locais naturais $\left(q^{i}, p_{i}\right)$ no fibrado cotangente $T^{*} Q$ de uma variedade $Q$, no caso simplético e, de forma totalmente análoga, as coordenadas locais adaptadas $\left(x^{\mu}, q^{i}, p_{i}^{\mu}, p\right)$ no fibrado dos cojatos estendido $J^{\oplus} E$ de um fibrado $E$ sobre $M$ (veja a equação (1.82)).

Passando a referenciais não-holônomos, continuaremos usando a mesma terminologia, chamando um referencial qualquer um referencial de Darboux se for adaptado à forma simplética ou multissimplética. Como veremos, referenciais não-holônomos de Darboux desempenham um papel importante neste trabalho e na definição de um tensor de Poisson.

Para construir o referencial não-holônomo em $J^{\circledast} E$ associado às três conexões mencionadas na hipótese do Teorema 2.2 e a um sistema qualquer $\left(x^{\mu}, q^{i}, p_{i}^{\mu}, p\right)$ de coordenadas locais adaptadas em $J^{\circledast} E$, procedemos como indicado acima, mas em três etapas. Primeiro, usamos a conexão $\Gamma_{E}$ em $E$ sobre $M$ para levantar os campos locais $\partial_{\mu}$ de $M$ para $E$ :

$$
\frac{\partial}{\partial x^{\mu}} \rightarrow \frac{\partial}{\partial x^{\mu}}+\Gamma_{\mu}^{i} \frac{\partial}{\partial q^{i}}
$$

Segundo, usamos a conexão $\Gamma_{\vec{J}^{\oplus} E}$ em $\vec{J}^{\circledast} E$ sobre $E$ para levantar os campos locais $\partial_{i}$ e $\partial_{\mu}$ 
de $E$ para $\vec{J}^{\otimes} E$ :

$$
\begin{gathered}
\frac{\partial}{\partial q^{i}} \rightarrow \frac{\partial}{\partial q^{i}}+\Gamma_{i j}^{k} p_{k}^{\nu} \frac{\partial}{\partial p_{j}^{\nu}} \\
\frac{\partial}{\partial x^{\mu}} \rightarrow \frac{\partial}{\partial x^{\mu}}-\left(\left(\partial_{j} \Gamma_{\mu}^{k}+\Gamma_{\mu}^{i} \Gamma_{i j}^{k}\right) p_{k}^{\nu}+\Gamma_{\mu \rho}^{\nu} p_{j}^{\rho}-\Gamma_{\mu \rho}^{\rho} p_{j}^{\nu}\right) \frac{\partial}{\partial p_{j}^{\nu}}
\end{gathered}
$$

Terceiro, usamos a conexão $\bar{\Gamma}_{\vec{J}^{\oplus} E}$ em $J^{\circledast} E$ sobre $\vec{J}^{\circledast} E$ para levantar os campos locais $\partial_{\mu}^{i}, \partial_{i}$ e $\partial_{\mu}$ de $\vec{J}^{\otimes} E$ para $J^{\circledast} E$ :

$$
\begin{gathered}
\frac{\partial}{\partial p_{i}^{\mu}} \rightarrow \frac{\partial}{\partial p_{i}^{\mu}}-\Gamma_{\mu}^{i} \frac{\partial}{\partial p}, \\
\frac{\partial}{\partial q^{i}} \rightarrow \frac{\partial}{\partial q^{i}}-\partial_{i} \Gamma_{\nu}^{j} p_{j}^{\nu} \frac{\partial}{\partial p}, \\
\frac{\partial}{\partial x^{\mu}} \rightarrow \frac{\partial}{\partial x^{\mu}}-\left(\partial_{\mu} \Gamma_{\nu}^{j} p_{j}^{\nu}-\Gamma_{\mu \rho}^{\rho}\left(p+\Gamma_{\nu}^{j} p_{j}^{\nu}\right)\right) \frac{\partial}{\partial p} .
\end{gathered}
$$

Combinando estas três etapas, chegamos ao seguinte teorema:

Definição 2.7 Dadas uma conexão $\Gamma_{E}$ em $E$ sobre $M$, uma conexão linear parcial $\Gamma_{V E}^{V E}$ em $V E$ sobre $E$ ao longo de $V E$ e uma conexão linear $\Gamma_{M}$ em $M$, o referencial de Darboux associado a este conjunto de conexóes e a um sistema $\left(x^{\mu}, q^{i}, p_{i}^{\mu}, p\right)$ de coordenadas locais adaptadas em $J^{\oplus E}$ é o referencial $\left(e_{\mu}, e_{i}, e_{\mu}^{i}, e_{0}\right)$ no fibrado tangente ou o referencial $\left(e^{\mu}, e^{i}, e_{i}^{\mu}, e^{0}\right)$ no fibrado cotangente de $J^{\oplus} E$ dado por

$$
\begin{gathered}
e_{0}=\frac{\partial}{\partial p}, \\
e_{\mu}^{i}=\frac{\partial}{\partial p_{i}^{\mu}}-\Gamma_{\mu}^{i} \frac{\partial}{\partial p}, \\
e_{i}=\frac{\partial}{\partial q^{i}}+\Gamma_{i j}^{r} p_{r}^{\nu} \frac{\partial}{\partial p_{j}^{\nu}}-\left(\partial_{i} \Gamma_{\nu}^{r}+\Gamma_{i j}^{r} \Gamma_{\nu}^{j}\right) p_{r}^{\nu} \frac{\partial}{\partial p}, \\
e_{\mu}=\frac{\partial}{\partial x^{\mu}}+\Gamma_{\mu}^{j} \frac{\partial}{\partial q^{j}}-\left(\partial_{j} \Gamma_{\mu}^{r} p_{r}^{\nu}+\Gamma_{\mu \rho}^{\nu} p_{j}^{\rho}-\Gamma_{\mu \rho}^{\rho} p_{j}^{\nu}\right) \frac{\partial}{\partial p_{j}^{\nu}} \\
-\left(\partial_{\mu} \Gamma_{\nu}^{j} p_{j}^{\nu}+\Gamma_{\mu}^{i} \partial_{i} \Gamma_{\nu}^{r} p_{r}^{\nu}-\Gamma_{\nu}^{j} \partial_{j} \Gamma_{\mu}^{r} p_{r}^{\nu}-\Gamma_{\mu \rho}^{\nu} \Gamma_{\nu}^{j} p_{j}^{\rho}-\Gamma_{\mu \rho}^{\rho} p\right) \frac{\partial}{\partial p},
\end{gathered}
$$




$$
\begin{gathered}
e^{\mu}=d x^{\mu}, \\
e^{i}=d q^{i}-\Gamma_{\mu}^{i} d x^{\mu}, \\
e_{i}^{\mu}=d p_{i}^{\mu}-\Gamma_{j i}^{r} p_{r}^{\mu} d q^{j}+\left(\left(\partial_{i} \Gamma_{\nu}^{r}+\Gamma_{j i}^{r} \Gamma_{\nu}^{j}\right) p_{r}^{\mu}+\Gamma_{\nu \rho}^{\mu} p_{i}^{\rho}-\Gamma_{\nu \rho}^{\rho} p_{i}^{\mu}\right) d x^{\nu}, \\
e^{0}=d p+\Gamma_{\mu}^{i} d p_{i}^{\mu}+\partial_{i} \Gamma_{\mu}^{r} p_{r}^{\mu} d q^{i}+\left(\partial_{\mu} \Gamma_{\nu}^{i} p_{i}^{\nu}-\Gamma_{\mu \rho}^{\rho}\left(p+\Gamma_{\nu}^{i} p_{i}^{\nu}\right)\right) d x^{\mu} .
\end{gathered}
$$

Uma demonstração independente de que estas expressões realmente definem referenciais covariantes, no sentido de que sob transformações locais de coordenadas adaptadas $\left(x^{\mu}, q^{i}, p_{i}^{\mu}, p\right) \rightarrow\left(x^{\prime \kappa}, q^{\prime k}, p_{k}^{\prime \kappa}, p^{\prime}\right)$ em $J^{\circledast} E$, de acordo com

$$
p_{k}^{\prime \kappa}=p_{k}^{\prime \kappa}\left(x^{\mu}, q^{i}, p_{i}^{\mu}\right), p^{\prime}=p^{\prime}\left(x^{\mu}, q^{i}, p_{i}^{\mu}, p\right),
$$

onde

$$
p_{k}^{\prime \kappa}=\operatorname{det}\left(\frac{\partial x}{\partial x^{\prime}}\right) \frac{\partial x^{\prime \kappa}}{\partial x^{\mu}} \frac{\partial q^{i}}{\partial q^{\prime k}} p_{i}^{\mu} \quad, \quad p^{\prime}=\operatorname{det}\left(\frac{\partial x}{\partial x^{\prime}}\right)\left(p-\frac{\partial q^{\prime k}}{\partial x^{\mu}} \frac{\partial q^{i}}{\partial q^{\prime k}} p_{i}^{\mu}\right)
$$

estes objetos satisfazem a simples lei de transformação $e_{0} \rightarrow e_{0}^{\prime}, e_{\mu}^{i} \rightarrow e_{\kappa}^{\prime k}, e_{i} \rightarrow e_{k}^{\prime}, e_{\mu} \rightarrow e_{\kappa}^{\prime}$ e $e^{0} \rightarrow e^{\prime 0}, e_{i}^{\mu} \rightarrow e_{k}^{\prime \kappa}, e^{i} \rightarrow e^{\prime k}, e^{\mu} \rightarrow e^{\prime \kappa}$ com

$$
\begin{gathered}
e_{0}^{\prime}=\operatorname{det}\left(\frac{\partial x^{\prime}}{\partial x}\right) e_{0}, e_{\kappa}^{\prime k}=\operatorname{det}\left(\frac{\partial x^{\prime}}{\partial x}\right) \frac{\partial x^{\mu}}{\partial x^{\prime \kappa}} \frac{\partial q^{\prime k}}{\partial q^{i}} e_{\mu}^{i} \\
e_{k}^{\prime}=\frac{\partial q^{i}}{\partial q^{\prime k}} e_{i}, e_{\kappa}^{\prime}=\frac{\partial x^{\mu}}{\partial x^{\prime \kappa}} e_{\mu}
\end{gathered}
$$

$\mathrm{e}$

$$
\begin{gathered}
e^{\prime 0}=\operatorname{det}\left(\frac{\partial x}{\partial x^{\prime}}\right) e^{0}, \quad e_{k}^{\prime \kappa}=\operatorname{det}\left(\frac{\partial x}{\partial x^{\prime}}\right) \frac{\partial x^{\prime \kappa}}{\partial x^{\mu}} \frac{\partial q^{i}}{\partial q^{\prime k}} e_{i}^{\mu}, \\
e^{\prime k}=\frac{\partial q^{\prime k}}{\partial q^{i}} e^{i}, e^{\prime \kappa}=\frac{\partial x^{\prime \kappa}}{\partial x^{\mu}} e^{\mu},
\end{gathered}
$$

encontra-se na segunda seção do Apêndice; uma afirmação análoga vale para o referencial no fibrado cotangente obtido por dualização.

O motivo principal para o uso do termo "referencial de Darboux" reside no fato de que em qualquer referencial de Darboux, a forma multissimplética $\omega$ assume a sua forma padrão: 
Teorema 2.3 Dadas uma conexão $\Gamma_{E}$ em E sobre $M$, uma conexão linear parcial $\Gamma_{V E}^{V E}$ em VE sobre $E$ ao longo de $V E$ sem torção e uma conexão linear $\Gamma_{M}$ em $M$ sem torção, então em qualquer referencial de Darboux associado a este conjunto de conexôes, a forma multissimplética w assume a forma padrão

$$
\omega=e^{i} \wedge e_{i}^{\mu} \wedge e_{\mu}^{n}-e^{0} \wedge e^{n},
$$

enquanto que a forma multicanônica $\theta$ assume a forma

$$
\theta=p_{i}^{\mu} e^{i} \wedge e_{\mu}^{n}+\left(p+\Gamma_{\mu}^{i} p_{i}^{\mu}\right) e^{n},
$$

onde $e^{n}$ denota o produto exterior dos $e^{\mu}$ e $e_{\mu}^{n}=i_{e_{\mu}} e^{n}$.

Demionstraç̃̃o. Calculamos

$$
\begin{aligned}
e^{i} \wedge e_{i}^{\mu} \wedge & e_{\mu}^{n}-e^{0} \wedge e^{n} \\
= & \left(d q^{i}-\Gamma_{\kappa}^{i} d x^{\kappa}\right) \wedge\left(d p_{i}^{\mu}-\Gamma_{j i}^{r} p_{r}^{\mu} d q^{j}\right. \\
& \left.\quad+\left(\left(\partial_{i} \Gamma_{\lambda}^{r}+\Gamma_{j i}^{r} \Gamma_{\lambda}^{j}\right) p_{r}^{\mu}+\Gamma_{\lambda \rho}^{\mu} p_{i}^{\rho}-\Gamma_{\lambda \rho}^{\rho} p_{i}^{\mu}\right) d x^{\lambda}\right) \wedge d^{n} x_{\mu} \\
& \quad-\left(d p+\Gamma_{\mu}^{i} d p_{i}^{\mu}+\partial_{i} \Gamma_{\mu}^{r} p_{r}^{\mu} d q^{i}\right) \wedge d^{n} x \\
= & d q^{i} \wedge d p_{i}^{\mu} \wedge d^{n} x_{\mu}-\Gamma_{j i}^{r} p_{r}^{\mu} d q^{i} \wedge d q^{j} \wedge d^{n} x_{\mu} \\
& +\left(\left(\partial_{i} \Gamma_{\mu}^{r}+\Gamma_{j i}^{r} \Gamma_{\mu}^{j}\right) p_{r}^{\mu}+\Gamma_{\mu \rho}^{\mu} p_{i}^{\rho}-\Gamma_{\mu \rho}^{\rho} p_{i}^{\mu}\right) d q^{i} \wedge d^{n} x \\
& +\Gamma_{\mu}^{i} d p_{i}^{\mu} \wedge d^{n} x-\Gamma_{\mu}^{i} \Gamma_{j i}^{r} p_{r}^{\mu} d q^{j} \wedge d^{n} x \\
& \quad-d p \wedge d^{n} x-\Gamma_{\mu}^{i} d p_{i}^{\mu} \wedge d^{n} x-\partial_{i} \Gamma_{\mu}^{r} p_{r}^{\mu} d q^{i} \wedge d^{n} x \\
= & \omega-\Gamma_{j i}^{r} p_{r}^{\mu} d q^{i} \wedge d q^{j} \wedge d^{n} x_{\mu}+\left(\Gamma_{\mu \rho}^{\mu} p_{j}^{\rho}-\Gamma_{\mu \rho}^{\rho} p_{j}^{\mu}\right) d q^{i} \wedge d^{n} x .
\end{aligned}
$$

Ao contrário da forma multissimplética $\omega$ e da forma multicanônica $\theta$, o tensor de Poisson $\pi$ depende explicitamente das conexões escolhidas.

Definição 2.8 Dadas uma conexão $\Gamma_{E}$ em $E$ sobre $M$, uma conexão linear parcial $\Gamma_{V E}^{V E}$ em VE sobre $E$ ao longo de VE sem torção e uma conexão linear $\Gamma_{M}$ em $M$ sem torção, definimos o correspondente tensor de Poisson, em qualquer referencial de Darboux associado a este conjunto de conexóes, por

$$
\pi_{\Gamma}=e_{i} \wedge e_{\mu}^{i} \wedge e_{n}^{\mu}-e_{0} \wedge e_{n},
$$

onde $e_{n}$ denota o produto exterior dos $e_{\mu}$ e $e_{n}^{\mu}=i_{e^{\mu}} e_{n}$. 
Observe que a definição de $\pi_{\Gamma}$ depende do conjunto de conexões escolhido mas independe do sistema de coordenadas locais adaptadas utilizadas na construção do referencial de Darboux associado, como pode ser facilmente verificado usando as leis de transformação (2.144) e (2.145).

Teorema 2.4 A diferença entre os tensores de Poisson $\pi_{\Gamma}$ e $\pi_{\Gamma^{\prime}}$ associados a diferentes conjuntos de conexóes $\Gamma$ e $\Gamma^{\prime}$ é um campo multivetorial a valores no núcleo de $\omega$.

DemonstraÇão. Esta afirmação segue diretamente do fato de que podemos escrever o tensor de Poisson $\pi_{\Gamma}$ na forma

$$
\begin{aligned}
\pi_{\Gamma}= & \left(e_{i} \wedge e_{\mu}^{i}-e_{0} \wedge e_{\mu}\right) \wedge e_{n}^{\mu} \\
= & {\left[\left(\frac{\partial}{\partial q^{i}}+\Gamma_{i j}^{r} p_{r}^{\nu} \frac{\partial}{\partial p_{j}^{\nu}}-\left(\partial_{i} \Gamma_{\nu}^{r}+\Gamma_{i j}^{r} \Gamma_{\nu}^{j}\right) p_{r}^{\nu} \frac{\partial}{\partial p}\right) \wedge\left(\frac{\partial}{\partial p_{i}^{\mu}}-\Gamma_{\mu}^{i} \frac{\partial}{\partial p}\right)\right.} \\
& \left.\quad-\frac{\partial}{\partial p} \wedge\left(\frac{\partial}{\partial x^{\mu}}+\Gamma_{\mu}^{j} \frac{\partial}{\partial q^{j}}-\left(\partial_{j} \Gamma_{\mu}^{r} p_{r}^{\nu}+\Gamma_{\mu \rho}^{\nu} p_{j}^{\rho}-\Gamma_{\mu \rho}^{\rho} p_{j}^{\nu}\right) \frac{\partial}{\partial p_{j}^{\nu}}\right)\right] \wedge e_{n}^{\mu} \\
= & {\left[\frac{\partial}{\partial q^{i}} \wedge \frac{\partial}{\partial p_{i}^{\mu}}+\Gamma_{i j}^{r} p_{r}^{\nu} \frac{\partial}{\partial p_{j}^{\nu}} \wedge \frac{\partial}{\partial p_{i}^{\mu}}-\left(\partial_{i} \Gamma_{\nu}^{r}+\Gamma_{i j}^{r} \Gamma_{\nu}^{j}\right) p_{r}^{\nu} \frac{\partial}{\partial p} \wedge \frac{\partial}{\partial p_{i}^{\mu}}\right.} \\
& \quad-\Gamma_{\mu}^{i} \frac{\partial}{\partial q^{i}} \wedge \frac{\partial}{\partial p}-\Gamma_{\mu}^{i} \Gamma_{i j}^{r} p_{r}^{\nu} \frac{\partial}{\partial p_{j}^{\nu}} \wedge \frac{\partial}{\partial p}-\frac{\partial}{\partial p} \wedge \frac{\partial}{\partial x^{\mu}}-\Gamma_{\mu}^{j} \frac{\partial}{\partial p} \wedge \frac{\partial}{\partial q^{j}} \\
& \left.+\left(\partial_{j} \Gamma_{\mu}^{r} p_{r}^{\nu}+\Gamma_{\mu \rho}^{\nu} p_{j}^{\rho}-\Gamma_{\mu \rho}^{\rho} p_{j}^{\nu}\right) \frac{\partial}{\partial p} \wedge \frac{\partial}{\partial p_{j}^{\nu}}\right] \wedge e_{n}^{\mu} \\
= & \left.\frac{\partial}{\partial q^{i}} \wedge \frac{\partial}{\partial p_{i}^{\mu}}-\frac{\partial}{\partial p} \wedge \frac{\partial}{\partial x^{\mu}}\right] \wedge \partial_{n} x^{\mu}+(\text { termos a valores no núcleo de } \omega),
\end{aligned}
$$

onde usamos a abreviação

$$
\partial_{n} x^{\mu}=\frac{1}{(n-1) !} \epsilon^{\mu \mu_{2} \ldots \mu_{n}} \frac{\partial}{\partial x^{\mu_{2}}} \wedge \ldots \wedge \frac{\partial}{\partial x^{\mu_{n}}} .
$$

sendo que o mesmo vale para o tensor de Poisson $\pi_{\Gamma^{\prime}}$. 



\section{Formalismo funcional}

A nossa meta neste capítulo será apresentar um resultado que estabelece uma conexão inédita entre o colchete de Poisson do formalismo multissimplético, conforme exposto no Capítulo 1, e o colchete de Peierls-DeWitt, que segundo [14,34] pode ser considerado o colchete de Poisson do formalismo funcional baseado no conceito do espaço de fase covariante. Na primeira seção, recordamos brevemente alguns aspectos deste formalismo e da sua conexão com o formalismo multissimplético. Na segunda seção, introduzimos uma classe de funcionais $F$ que são definidos a partir de formas diferenciais $f$ sobre o espaço de multifase do formalismo multissimplético por pull-back e subsequente integração sobre alguma subvariedade do espaço-tempo. Na terceira seção, provamos o teorema principal desta tese, segundo o qual o campo hamiltoniano funcional $X_{F}$ associado ao funcional $F$ pode, em certas circunstâncias, ser obtido diretamente, de forma algébrica, do campo hamiltoniano $X_{f}$ associado à forma $f$, sem a necessidade de introduzir a função de Green causal associada às equações de movimento linearizadas para definir $X_{F}$ por uma integral de convolução, como em [14,34]. Como corolário, obtemos pela primeira vez uma relação direta entre o colchete de Poisson do formalismo multissimplético e o colchete de Peierls-DeWitt do formalismo funcional.

\subsection{Espaço de fase covariante}

O espaço de fase covariante na teoria dos campos é, por definição, o espaço das soluções das equações de movimento - ao contrário do espaço de fase tradicional, que é definido em termos de dados de Cauchy, o que ofusca a covariância da teoria. Obviamente, não é de se esperar que estas duas interpretações do espaço de fase sejam equivalentes de forma completamente geral, pois é bem conhecido que, para equações de movimento não-lineares, a evolução temporal a partir de dados de Cauchy regulares pode gerar soluções que, dentro de um tempo finito, desenvolvem algum tipo de singularidade. Um pré-requisito ainda mais 
elementar é que o espaço-tempo subjacente $M$ deva admitir pelo menos uma hipersuperfície de Cauchy, o que significa que $M$ deva ser globalmente hiperbólico.

Portanto, uma das hipóteses fundamentais neste capítulo será que o espaço-tempo $M$ seja globalmente hiperbólico. É esta a arena natural para a teoria matemática de (sistemas de) equações diferenciais parciais hiperbólicas, onde o problema de Cauchy é bem posto. Existem várias e aparentemente diferentes definições do conceito de um espaço-tempo globalmente hiperbólico, mas no final todas se mostraram equivalentes; veja [38, Cap. 8] para uma extensa discussão. A mais conveniente para os nossos fins é que $M$ admite uma função tempo global ${ }^{1}$ cujas hipersuperfícies de nível providenciam uma folheação de $M$ em superfícies de Cauchy e um difeomorfismo global $M \cong \mathbb{R} \times \Sigma$. Como corolário imediato, podemos definir a noção de uma fatia temporal (aberta ou fechada) em $M$ : é um subconjunto (aberto ou fechado) de $M$ que sob tal difeomorfismo corresponde a um subconjunto de $\mathbb{R} \times \Sigma$ da forma $I \times \Sigma$, onde $I$ é um intervalo (aberto ou fechado) em $\mathbb{R}$.

Dentro da abordagem adotada neste trabalho, onde campos são seções de fibrados, é natural considerar o espaço de fase covariante $\mathcal{S}$ como inserido no espaço de configurações de campo $\mathcal{C}$, que por sua vez é o espaço das seções de um certo fibrado $F$ :

$$
\mathcal{C}=\Gamma(F) \text {. }
$$

No formalismo desenvolvido no Capítulo $1, F$ é o fibrado $E$ no âmbito lagrangiano e é o fibrado $\vec{J}{ }^{\circledast} E$ no âmbito hamiltoniano. Destarte, os pontos de $\mathcal{C}$, que são as seções $\phi$ de $F$, correspondem a seções $\varphi$ de $E$ no âmbito lagrangiano e a seções $(\varphi, \pi)$ de $\overrightarrow{J^{\circledast}} E$ no âmbito hamiltoniano. Em ambos os casos, os elementos de $\mathcal{S}$ são os pontos estacionários do funcional ação, que em cada ponto $\phi$ de $\mathcal{C}$ é definido como a integral da forma $\phi^{*} \theta_{\mathcal{L}}$ no âmbito lagrangiano e da forma $\phi^{*} \theta_{\mathcal{H}}$ no âmbito hamiltoniano. As correspondentes equações de movimento constituem um sistema de equações diferenciais parciais, conhecidas como equações de Euler - Lagrange no âmbito lagrangiano e como equações de De Donder-Weyl no âmbito hamiltoniano.

Formalmente, o espaço de configurações de campo $C$ pode ser visto como uma variedade (de dimensão infinita). Como tal, possui em cada um dos seus pontos um espaço tangente $T_{\phi} \mathrm{C}$ que formalmente pode ser definido como um espaço de seções lisas, com certas propriedades de suporte, do fibrado vetorial $V_{\phi}=\phi^{*}(V F)$ sobre $M$. Denotando por $V_{\phi}^{*}=\phi^{*}\left(V^{*} F\right)$ 。 dual comum e por $V_{\phi}^{\circledast}=V_{\phi}^{*} \otimes \Lambda^{n} T^{*} M$ o dual torcido de $V_{\phi}$, vimos que o espaço cotangente será então o espaço das seções distribucionais, com propriedades de suporte duais, do fibrado vetorial $V_{\phi}^{\circledast}$ sobre $M$. Existem várias opções para a escolha das propriedades de suporte a serem impostas, entre as quais adotaremos a opção 3 de [14]:

$$
T_{\phi} \mathrm{C}=\Gamma_{\mathrm{sc}}^{\infty}\left(V_{\phi}\right), T_{\phi}^{*} \mathrm{C}=\Gamma_{\mathrm{tc}}^{-\infty}\left(V_{\phi}^{\circledast}\right)
$$

\footnotetext{
${ }^{1}$ É claro que tal função tempo está longe de ser única - mesmo no espaço-tempo de Minkowski.
} 
onde os índices "sc" e "tc" significam "suporte espacialmente compacto" e "suporte temporalmente compacto", respectivamente. Dizemos que uma seção de um fibrado vetorial sobre o espaço-tempo $M$ tem suporte espacialmente compacto se a interseção de seu suporte com qualquer fatia temporal fechada for compacta e que tem suporte temporalmente compacto se seu suporte for contido em alguma fatia temporal.

Dentro do espaço de configurações de campo $\mathcal{C}$, temos o espaço de fase covariante $\mathcal{S}$, que pode ser definido como o conjunto dos pontos estacionários do funcional ação $S$ :

$$
\mathcal{S}=\left\{\phi \in \mathcal{C} / S^{\prime}[\phi]=0\right\} .
$$

Formalmente, $\mathcal{S}$ pode ser visto como subvariedade de $\mathcal{C}$. Portanto, possui em cada um dos seus pontos um espaço tangente $T_{\phi} \mathcal{S}$ que é o subespaço do espaço tangente $T_{\phi} \mathcal{C}$ consistindo das soluções das equações de movimento linearizadas (onde "linearizadas" significa, mais precisamente, "linearizadas em torno da solução $\phi$ das plenas equações de movimento"). Estas soluções são exatamente as seções de $V_{\phi}$ que pertencem ao núcleo do correspondente operador de Jacobi $\partial[\phi]: \Gamma\left(V_{\phi}\right) \longrightarrow \Gamma\left(V_{\phi}^{\circledast}\right)$ :

$$
T_{\phi} \mathcal{S}=\left\{\delta \phi \in T_{\phi} \mathcal{C} / \partial[\phi] \cdot \delta \phi=0\right\} .
$$

Para maiores detalhes, inclusive uma definição explícita do operador de Jacobi, tanto no âmbito lagrangiano como no âmbito hamiltoniano, veja Ref. [14].

A estrutura simplética sobre o espaço de fase covariante é descrita por uma forma simplética funcional $\Omega$ que associa a todo ponto $\phi \mathrm{em} \mathcal{S}$ e quaisquer dois vetores tangentes $\delta \phi_{1}$ e $\delta \phi_{2}$ em $T_{\phi} \mathcal{\delta}$ um número real $\Omega_{\phi}\left(\delta \phi_{1}, \delta \phi_{2}\right)$. Como foi observado por Crnković e Witten [4,5], este pode ser escrito como a integral de uma "corrente simplética", que é uma $(n-1)$-forma fechada sobre o espaço-tempo dependendo de $\phi, \delta \phi_{1}$ e $\delta \phi_{2}$, sobre uma hipersuperfície $\Sigma$ de Cauchy qualquer:

$$
\Omega_{\phi}\left(\delta \phi_{1}, \delta \phi_{2}\right)=\int_{\Sigma} J_{\phi}\left(\delta \phi_{1}, \delta \phi_{2}\right)
$$

No formalismo multissimplético, esta "corrente simplética" pode ser construída diretamente a partir da forma multissimplética em termos de um pull-back modificado, em dois passos. No âmbito lagrangiano, o primeiro passo consiste em usar a lagrangiana $\mathcal{L}$, ou mais exatamente a transformação de Legendre $\mathbb{F} \mathcal{L}: J E \rightarrow J^{\circledast} E$ induzida pela lagrangiana $\mathcal{L}$, para definir a forma de Poincaré-Cartan $\omega_{\mathcal{L}}=(\mathbb{F} \mathcal{L})^{*} \omega$ sobre $J E$. No segundo passo, define-se um "pull-back misturado" de $\omega_{\mathcal{L}}$ pelas derivadas $j \phi$ de $\phi, j\left(\delta \phi_{1}\right)$ de $\delta \phi_{1}$ e $j\left(\delta \phi_{2}\right)$ de $\delta \phi_{2}$, onde nos primeiros dois argumentos de $\omega_{\mathcal{L}}$, inserimos $j\left(\delta \phi_{1}\right)$ e $j\left(\delta \phi_{2}\right)$, respectivamente, ao invés da aplicação tangente $T j(\phi)$ a $j(\phi)$ que aparece nos demais $n-1$ argumentos. De forma análoga, no âmbito hamiltoniano, o primeiro passo consiste em usar a hamiltoniana $\mathcal{H}$ para definir a forma de De Donder-Weyl $\omega_{\mathcal{H}}=\mathcal{H}^{*} \omega$ sobre $\vec{J}^{\circledR} E$. No segundo passo, define-se um "pull-back misturado" de $\omega_{\mathcal{H}}$ por $\phi, \delta \phi_{1}$ e $\delta \phi_{2}$, onde nos primeiros dois argumentos de $\omega_{\mathcal{H}}$, 
inserimos $\delta \phi_{1}$ e $\delta \phi_{2}$, respectivamente, ao invés da aplicação tangente $T \phi$ a $\phi$ que aparece nos demais $n-1$ argumentos. Explicitamente, no âmbito hamiltoniano em que a construção é bem mais simples, temos

$$
\left(\phi^{*} \omega_{\mathcal{H}}\left(\delta \phi_{1}, \delta \phi_{2}\right)\right)_{x}\left(u_{1}, \ldots, u_{n-1}\right)=\left(\omega_{\mathcal{H}}\right)_{\phi(x)}\left(\delta \phi_{1}(x), \delta \phi_{2}(x), T_{x} \phi \cdot u_{1}, \ldots, T_{x} \phi \cdot u_{n-1}\right),
$$

e então

$$
J_{\phi}\left(\delta \phi_{1}, \delta \phi_{2}\right)=\phi^{*} \omega_{\mathcal{H}}\left(\delta \phi_{1}, \delta \phi_{2}\right) .
$$

Em coordenadas locais adaptadas, escrevendo

$$
J_{\phi}\left(\delta \phi_{1}, \delta \phi_{2}\right)=J_{\phi}^{\mu}\left(\delta \phi_{1}, \delta \phi_{2}\right) d^{n} x_{\mu}
$$

e

$$
\delta \phi_{1}=\delta \varphi_{1}^{i} \frac{\partial}{\partial q^{i}}+\delta \pi_{1, i}^{\mu} \frac{\partial}{\partial p_{i}^{\mu}} \quad, \quad \delta \phi_{2}=\delta \varphi_{2}^{i} \frac{\partial}{\partial q^{i}}+\delta \pi_{2, i}{ }^{\mu} \frac{\partial}{\partial p_{i}^{\mu}},
$$

obtemos

$$
\Omega_{\phi}\left(\delta \phi_{1}, \delta \phi_{2}\right)=\int_{\Sigma} d \Sigma_{\mu} J_{\phi}^{\mu}\left(\delta \phi_{1}, \delta \phi_{2}\right)
$$

com

$$
J_{\phi}^{\mu}\left(\delta \phi_{1}, \delta \phi_{2}\right)=\delta \varphi_{1}^{i} \delta \pi_{2, i}{ }^{\mu}-\delta \varphi_{2}^{i} \delta \pi_{1, i}^{\mu}
$$

Aliás, estas fórmulas mostram que, exatamente como na mecânica, $\Omega$ não depende da escolha da hamiltoniana $\mathcal{H}$ (o mesmo vale para $J$ ).

Continuando a discussão sobre como desenvolver uma versão formal da geometria simplética no espaço de fase covariante, o próximo passo seria introduzir o análogo de funções, de suas derivadas, do campo hamiltoniano associado a uma função e, finalmente, do colchete de Poisson entre duas funções. Como $\mathcal{S}$ é de certa forma um espaço de funções e portanto o análogo das funções sobre um espaço de fase comum aqui são os funcionais sobre $\mathcal{S}$, todos estes conceitos adquirirão um adjetivo "funcional".

A primeira destas construções é a da derivada funcional. Dado um funcional $F$ sobre $\mathcal{C}$, a sua derivada funcional no ponto $\phi$ em $\mathcal{C}$ ao longo do vetor tangente funcional $\delta \phi \in T_{\phi} \mathcal{C}$ é definida por

$$
F^{\prime}[\phi] \cdot \delta \phi=\left.\frac{d}{d \lambda} F\left[\phi_{\lambda}\right]\right|_{\lambda=0}
$$

onde os $\phi_{\lambda}$ representam uma família a um parâmetro de seções de $F$ tal que

$$
\phi=\left.\phi_{\lambda}\right|_{\lambda=0}, \quad \delta \phi=\left.\frac{d}{d \lambda} \phi_{\lambda}\right|_{\lambda=0} .
$$

Para que esta prescrição realmente defina um vetor cotangente funcional $\digamma^{\prime}[\phi] \in T_{\phi}^{*} \mathrm{C}$, será necessário garantir compatibilidade com as propriedades de suporte estipulados na 
equação (3.2). Portanto, neste trabalho consideraremos exclusivamente funcionais que são "locais no tempo", i.e., cuja dependência dos campos é não-trivial apenas dentro de uma fatia temporal, ou em outras palavras, cuja derivada funcional tem suporte temporalmente compacto. (Os funcionais a serem estudados nas próximas seções tem esta propriedade, uma vez que são definidos como integrais de expressões locais nos campos sobre alguma hipersuperfície de Cauchy.) Observamos também que $F^{\prime}[\phi]$ é uma seção distribucional do fibrado vetorial $V_{\phi}^{\circledast}$ para a qual utilizaremos a seguinte notação padrão,

$$
F^{\prime}[\phi] \cdot \delta \phi=\int_{M} d^{n} x \frac{\delta F}{\delta \phi^{i}}[\phi](x) \delta \phi^{i}(x)
$$

onde podemos considerar que, localmente,

$$
\frac{\delta F}{\delta \phi}[\phi] \in \Gamma_{\mathrm{tc}}^{-\infty}\left(V_{\phi}^{*}\right) \quad, \quad \frac{\delta F}{\delta \phi}[\phi] d^{n} x \in \Gamma_{\mathrm{tc}}^{-\infty}\left(V_{\phi}^{\circledast}\right) .
$$

As mesmas considerações se aplicam, "mutatis mutandis", se substituírmos o espaço de configurações de campo $\mathcal{C}$ pelo espaço de fase covariante $\mathcal{S}$. Observamos apenas que o fato de que os elementos $\delta \phi$ de $T_{\phi} \mathcal{S}$ devem satisfazer (sistemas de) equações diferenciais parciais, tipicamente de caráter hiperbólico, exclui a opção de exigir que eles tenham suporte compacto ou suporte temporalmente compacto e motiva a condição formulada na equação (3.2) de que tenham suporte espacialmente compacto como a única condição de suporte consistente com as referidas equações de evolução.

O problema básico a ser resolvido para poder implementar a filosofia da geometria simplética dentro do formalismo funcional é conseguir dualizar, em cada ponto $\phi$ de $\mathcal{S}$, o covetor funcional $F^{\prime}[\phi]$, em relação à forma simplética funcional $\Omega_{\phi}$, para obter um vetor funcional $X_{F}[\phi]$. Formalmente, a definição de $X_{F}[\phi]$ em termos de $F^{\prime}[\phi]$ é dada pela fórmula

$$
\Omega_{\phi}\left(X_{F}[\phi], \delta \phi\right)=F^{\prime}[\phi] \cdot \delta \phi \quad \text { para todo } \delta \phi \in T_{\phi} \mathcal{S}
$$

Observa-se que, ao contrário do que acontece em variedades simpléticas de dimensão finita, esta relação não garante que $X_{F}[\phi]$ pertença ao espaço $T_{\phi} \mathcal{S}$. De fato, inspecionando a definição de $\Omega_{\phi}\left(\delta \phi_{1}, \delta \phi_{2}\right)$, percebe-se que basta supor que uma das duas seções $\delta \phi_{1}$ e $\delta \phi_{2}$ seja lisa e tenha suporte espacialmente compacto, sendo que a outra pode então ser distribucional e ter suporte qualquer, e mesmo assim a expressão $\Omega_{\phi}\left(\delta \phi_{1}, \delta \phi_{2}\right)$ será bem definida. Portanto, é de se esperar que, de forma geral, não valha

$$
X_{F}[\phi] \in \Gamma_{\mathrm{sc}}^{\infty}\left(V_{\phi}\right),
$$

mas que valha apenas

$$
X_{F}[\phi] \in \Gamma^{-\infty}\left(V_{\phi}\right) .
$$


É neste sentido "fraco" que é possível exibir, no contexto do espaço de fase covariante, o campo hamiltoniano funcional $X_{F}$ associado ao funcional $F$.

O resultado principal da tese [34] afirma que $X_{F}[\phi]$ pode ser construído explicitamente como convolução da distribuição que define a derivada funcional de $F$ em $\phi$ com a função de Green causal $G_{\phi}$ do operador de Jacobi em $\phi$ :

$$
\left(X_{F}[\phi]\right)^{i}(x)=\int_{M} d^{n} y G_{\phi}^{i j}(x, y) \frac{\delta F}{\delta \phi^{j}}[\phi](y) .
$$

Observa-se que esta convolução é bem definida, pois quando fixarmos $x, G_{\phi}(x,$.$) é uma$ distribuição de suporte espacialmente compacto em $y$ enquanto que, por hipótese, $\delta F / \delta \phi[\phi]$ é uma distribuição de suporte temporalmente compacto, e a interseção dos dois suportes é compacto. Por outro lado, não há como fazer nenhuma afirmação sobre propriedades de regularidade ou de suporte da distribuição resultante.

Usando este resultado, é praticamente trivial escrever o colchete de Poisson induzido por $\Omega$ : Para dois funcionais $F$ e $G$,

$$
\{F, G\}[\phi]=F^{\prime}[\phi] \cdot X_{G}[\phi]=-G^{\prime}[\phi] \cdot X_{F}[\phi] .
$$

Explicitamente,

$$
\{F, G\}[\phi]=\int_{M} d^{n} x \int_{M} d^{n} y \frac{\delta F}{\delta \phi^{i}}(x) G_{\phi}^{i j}(x, y) \frac{\delta G}{\delta \phi^{j}}(y) .
$$

Este é o colchete de Peierls - DeWitt.

A desvantagem principal das fórmulas (3.17) e (3.19) é que elas ainda são relativamente implícitas e que dependem da dinâmica do modelo concreto, pois calcular $G_{\phi}$ requer resolver um sistema hiperbólico de equações diferenciais parciais lineares, onde a solução $\phi$ das equações de movimento aparece nos coeficientes do sistema.

O resultado principal da presente tese consistirá de uma fórmula muito mais simples e direta para calcular $X_{F}$, para uma classe restrita de funcionais que no entanto é suficientemente importante nas aplicações para merecer uma atenção especial. Para tais funcionais, veremos que o colchete de Peierls - DeWitt se reduz a uma versão modificada do colchete de Poisson discutido no Capítulo 1.

\subsection{Funcionais gerados por formas}

A nossa meta nesta seção será introduzir uma classe de funcionais no espaço de fase covariante que são definidos a partir de formas diferenciais sobre o espaço de multifase (estendido ou comum) do formalismo multissimplético por pull-back e integração, e calcular a derivada funcional de um tal funcional em termos da derivada de Lie da respectiva forma diferencial. 
Para simplificar o tratamento, restringiremo-nos ao formalismo hamiltoniano, observando que resultados completamente análogos no contexto do formalismo lagrangiano podem ser obtidos por pull-back via a transformação de Legendre $\overrightarrow{\mathbb{F}} \mathcal{L}$ pertinente, substituíndo $\vec{J}^{\circledast} E$ por $J E$ e $\mathcal{H}$ por $\mathbb{F} \mathcal{L}$.

Definição 3.1 Sejam $\Sigma$ uma subvariedade p-dimensional de $M$ e $f$ uma p-forma sobre o espaço de multifase comum $\vec{J}^{\circledast} E$. Então definimos o funcional $F$ associado a $\Sigma$ e $f$ pela fórmula

$$
F[\phi]=\int_{\Sigma} \phi^{*} f \quad \text { para } \phi \in \Gamma(\vec{J} \otimes E)
$$

A rigor, não especificamos aqui se queremos considerar $F$ como funcional sobre o espaço de fase covariante $\mathcal{S}$ ou sobre o espaço $\mathcal{C}$ das configurações de campo da teoria. De qualquer forma, a segunda opção constitui uma extensão da primeira opção que é definida naturalmente, sem qualquer arbitrariedade. Também deixamos de especificar condições para garantir a existência da integral, pois existem vários tipos de tais condições que são bem diferentes entre si. Em primeiro lugar, deve-se salientar que $\Sigma$ pode ser uma subvariedade com bordo. Desta forma, podemos garantir a existência da integral se supormos que $\Sigma$ seja compacta. Outra possibilidade consiste em impor restrições sobre $f$. Por exemplo, podemos supor que $f$ tenha suporte na base compacto (i.e., o conjunto dos pontos $x$ em $M$ tais que $f$ não se anula na fibra de $J^{\circledast} E$ sobre $x$ é compacto) ou que $f$ satisfaça condições apropriadas de decaimento ao infinito.

Outro ponto que merece ser mencionado é que dada uma hamiltoniana $\mathcal{H}$ qualquer, podemos sempre supor que $f$ seja o pull-back de uma $p$-forma sobre $J^{\oplus} E$ via $\mathcal{H}$, pois sempre vale $f=\mathcal{H}^{*}\left(\eta^{*} f\right)$; será esta a situação que encontraremos nas aplicações a seguir.

O próximo passo consiste em calcular a derivada funcional para essa classe de funcionais. Para tanto, lembramos primeiro que o espaço tangente formal $T_{\phi} \mathcal{C}$ de $\mathcal{C}$ no ponto $\phi$ de $\mathcal{C}$ é o espaço $\Gamma\left(\phi^{*}\left(V\left(\vec{J}^{\circledast} E\right)\right)\right)$ das seções $\delta \phi$ do pull-back do fibrado vertical $V\left(\vec{J}^{\circledast} E\right)$ de $\vec{J}{ }^{\circledast} E$ por $\phi$. Entre estas, temos as seções obtidas por composição de $\phi$ com um campo vetorial vertical $\vec{X}$ sobre $\vec{J}{ }^{\circledast} E$,

$$
\delta \phi(x)=\vec{X}(\phi(x)) .
$$

Usando o fato de que $\phi$ não é apenas uma aplicação qualquer de $M$ para $\vec{J}^{\circledast} E$ mas é uma seção de $\vec{J}^{\circledast} E$ como fibrado sobre $M$, pode-se provar que qualquer variação $\delta \phi \in \Gamma\left(\phi^{*}\left(V\left(\vec{J}^{\circledast} E\right)\right)\right)$ de $\phi$ pode ser escrita assim, para algum campo vetorial vertical $\vec{X} \in \mathfrak{X}_{V}\left(\vec{J}^{\circledast} E\right)$ sobre $\vec{J}^{\circledast} E$. (Para demonstrar esta afirmação, que vale não apenas para $\vec{J}^{\circledast} E$ para um fibrado $F$ sobre $M$ qualquer, aplicamos o teorema das funções inversas para construir, a partir de um sistema de coordenadas locais $\left(x^{\mu}, r^{k}\right)$ de $F$ em torno de um ponto qualquer $\phi(m)$ da imagem de $M$ em $F$ sob $\phi$, induzido por um sistema de coordenadas locais $x^{\mu}$ de $M$ em torno de $m$, um sistema de coordenadas locais $r^{k}$ da fibra típica de $F$ e uma trivialização local de $F$ sobre $M$ 
como sempre, um novo sistema de coordenadas locais $\left(x^{\mu}, s^{l}\right)$ de $F$ em torno de $\phi(m)$ tal que nele, a seção $\phi$ é representada por $s^{l}(x)=0$. Então usando uma função corte em torno da origem, é fácil estender as funções $\delta \phi^{l}(x)$ para funções $X^{l}(x, s)$. Finalmente, usa-se uma partição da unidade sobre $M$ para colar esta "extensões" locais de $\delta \phi$ e assim chegar a uma "extensão" global de $\delta \phi$.) Obviamente, $\vec{X}$ está longe de ser unicamente determinado por $\delta \phi$, já que apenas os valores de $\vec{X}$ sobre a imagem de $M$ em $\vec{J}{ }^{\circledast} E$ sob $\phi$ são determinados por $\delta \phi$, através da condição (3.21).

Com essa ferramenta de extensão de campos vetoriais (verticais) ao longo de uma seção de um fibrado para campos vetoriais (verticais) na varíedade alvo (o espaço total do fibrado) à nossa disposição, podemos calcular a derivada funcional de funcionais do tipo (3.20).

Proposição 3.1 Sejam $\Sigma$ uma subvariedade p-dimensional de $M$ e $f$ uma p-forma sobre o espaço de multifase comum $\vec{J}^{\otimes} E$. Então a derivada funcional do funcional $F$ associado $a$ $\Sigma$ e f conforme a equação (3.20) é dada pela fórmula

$$
F^{\prime}[\phi] \cdot \vec{X}(\phi)=\int_{\Sigma} \phi^{*}\left(L_{\vec{X}} f\right), \quad \text { para } \phi \in \Gamma\left(\vec{J}{ }^{\otimes} E\right) \text { e } \vec{X} \in \mathfrak{X}_{V}\left(\vec{J}^{\otimes} E\right) .
$$

DemonstraÇÃo. Lembremos que para um funcional $F$ qualquer, a sua derivada funcional em $\phi$ ao longo de $\delta \phi$ é definida por

$$
F^{\prime}[\phi] \cdot \delta \phi=\left.\frac{d}{d \lambda} F\left[\phi_{\lambda}\right]\right|_{\lambda=0},
$$

onde os $\phi_{\lambda}$ representam uma família a um parâmetro de seções de $\vec{J}^{\circledR} E$ tal que

$$
\phi=\left.\phi_{\lambda}\right|_{\lambda=0} \quad, \quad \delta \phi=\left.\frac{d}{d \lambda} \phi_{\lambda}\right|_{\lambda=0} .
$$

Fixando $\phi$ e $\delta \phi$, introduzimos um campo vetorial vertical $\vec{X}$ sobre $\vec{J}^{\otimes} E$ que representa a variação $\delta \phi$ de $\phi$, conforme a equação (3.21), e consideramos seu fluxo, que é um grupo (local) de automorfismos (locais) estritos $\Phi_{\lambda}$ de $\vec{J}^{\circledast} E$ tal que

Podemos então por

$$
\vec{X}=\left.\frac{d}{d \lambda} \Phi_{\lambda}\right|_{\lambda=0} \text {. }
$$

para concluir que

$$
\phi_{\lambda}=\Phi_{\lambda} \circ \phi
$$

$$
\begin{aligned}
F^{\prime}[\phi] \cdot \delta \phi & =\left.\int_{\Sigma} \frac{d}{d \lambda} \phi_{\lambda}^{*} f\right|_{\lambda=0}=\left.\int_{\Sigma} \frac{d}{d \lambda}\left(\Phi_{\lambda} \circ \phi\right)^{*} f\right|_{\lambda=0} \\
& =\left.\int_{\Sigma} \phi^{*} \frac{d}{d \lambda} \Phi_{\lambda}^{*} f\right|_{\lambda=0}=\int_{\Sigma} \phi^{*}\left(L_{\vec{X}} f\right) .
\end{aligned}
$$




\subsection{Os teoremas principais}

Inicialmente, formulamos um teorema que providencia uma interpretação global para as equações de movimento na teoria geométrica dos campos, em termos de um operador que podemos chamar de operador de Euler - Lagrange, no formalismo lagrangiano, e de operador de De Donder - Weyl, no formalismo hamiltoniano. Em ambos os casos, é possível deduzir as equações de movimento a partir de um princípio variacional. Como este procedimento é bem conhecido no formalismo lagrangiano, restringiremo-nos aqui a apresentar a sua versão hamiltoniana.

Definição 3.2 Dada uma hamiltoniana $\mathcal{H}$, definimos o correspondente operador de De Donder-Weyl como sendo a aplicacão

$$
\mathcal{D}_{\mathcal{H}}: J\left(\vec{J}^{\circledast} E\right) \longrightarrow T^{*}\left(\vec{J}^{\circledast} E\right) \otimes \bigwedge^{n} T^{*} M
$$

de fibrados sobre $\vec{J}^{\circledast} E^{2}$ que associa a cada jato de primeira ordem $(\varphi, \pi, \partial \varphi, \partial \pi)$ de seções (locais) $(\varphi, \pi)$ de $\vec{J}^{\circledast} E$ sobre $M$ e a cada campo vetorial $\vec{X}$ sobre $\vec{J}^{\circledast} E$ a $n$-forma sobre $M$ dada por

$$
\mathcal{D}_{\mathcal{H}}(\varphi, \pi, \partial \varphi, \partial \pi) \cdot \vec{X}=(\varphi, \pi)^{*}\left(i_{\vec{X}} \omega_{\mathcal{H}}\right)
$$

A importância deste operador decorre do seguinte

Teorema 3.1 Dadas uma hamiltoniana $\mathcal{H}$ e uma seção $\phi=(\varphi, \pi)$ de $\vec{J}^{\circledR} E$, as seguintes condiçôes são equivalentes:

- $\phi$ satisfaz as equações de De Donder-Weyl;

- $\phi$ é aniquilado pelo operador de De Donder-Weyl, ou seja, para todo campo vetorial $\vec{X}$ sobre $\vec{J}^{\otimes} E$, vale

$$
\mathcal{D}_{\mathcal{H}}(\varphi, \pi, \partial \varphi, \partial \pi) \cdot \vec{X}=0
$$

- $\phi$ é ponto estacionário do funcional ação

$$
S_{\mathcal{H}}[\phi]=\int_{M} \phi^{*} \theta_{\mathcal{H}}
$$

DemonstraÇÃo. Seja $\vec{X}$ um campo vetorial sobre $\vec{J}{ }^{\circledR} E$, cuja expressão em coordenadas locais adaptadas é dada pela equação (1.150). Calculando o pull-back da forma $i_{\vec{X}} \omega_{\mathcal{H}}$, que

\footnotetext{
${ }^{2}$ Suprimimos os simbolos que indicam o pull-back de fibrados sobre $E$ ou $M$ para $\vec{J}^{\circledast} E$.
} 
é dada pela equação $(1.151), \operatorname{com}(\varphi, \pi)$, obtemos

$$
\begin{aligned}
(\varphi, \pi)^{*}\left(i_{\vec{X}} \omega_{\mathcal{H}}\right)= & X^{\nu}(\varphi, \pi)\left(\partial_{\mu} \varphi^{i} \partial_{\nu} \pi_{i}^{\mu}-\partial_{\nu} \varphi^{i} \partial_{\mu} \pi_{i}^{\mu}\right. \\
& \left.-\partial_{\nu} \varphi^{i} \frac{\partial H}{\partial q^{i}}(\varphi, \pi)-\partial_{\nu} \pi_{i}^{\mu} \frac{\partial H}{\partial p_{i}^{\mu}}(\varphi, \pi)\right) d^{n} x \\
& +X^{i}(\varphi, \pi)\left(\frac{\partial H}{\partial q^{i}}(\varphi, \pi)+\partial_{\mu} \pi_{i}^{\mu}\right) d^{n} x \\
& +X_{i}^{\mu}(\varphi, \pi)\left(\frac{\partial H}{\partial p_{i}^{\mu}}(\varphi, \pi)-\partial_{\mu} \varphi^{i}\right) d^{n} x
\end{aligned}
$$

ou seja,

$$
\begin{aligned}
(\varphi, \pi)^{*}\left(i_{\vec{X}} \omega_{\mathcal{H}}\right)= & \left(\left(\frac{\partial H}{\partial q^{i}}(\varphi, \pi)+\partial_{\mu} \pi_{i}^{\mu}\right)\left(X^{i}(\varphi, \pi)-X^{\nu}(\varphi, \pi) \partial_{\nu} \varphi^{i}\right)\right. \\
& \left.+\left(\frac{\partial H}{\partial p_{i}^{\mu}}(\varphi, \pi)-\partial_{\mu} \varphi^{i}\right)\left(X_{i}^{\mu}(\varphi, \pi)-X^{\nu}(\varphi, \pi) \partial_{\nu} \pi_{i}^{\mu}\right)\right) d^{n} x
\end{aligned}
$$

demonstrando a equivalência entre o primeiro critério e o segundo critério. Note que as fórmulas (3.27) ou (3.28) mostram que a condição (3.25) vale para todo campo vetorial $\vec{X}$ sobre $\vec{J}{ }^{\otimes} E$ se vale para todo campo vetorial vertical $\vec{X}$ sobre $\vec{J}{ }^{\otimes} E$. Para demonstrar a equivalência entre o segundo critério, nesta forma, e o terceiro critério, utilizamos o result́ado da Proposição 3.1 , segundo o qual temos para todo campo vetorial vertical $\vec{X}$ sobre $\vec{J}^{\otimes} E$

$$
S_{\mathcal{H}}^{\prime}[\phi] \cdot \vec{X}(\phi)=\int_{M} \phi^{*}\left(L_{\vec{X}} \theta_{\mathcal{H}}\right)=\int_{M} \phi^{*}\left(i_{\vec{X}} d \theta_{\mathcal{H}}\right)+\int_{M} \phi^{*}\left(d i_{\vec{X}} \theta_{\mathcal{H}}\right) .
$$

Como o princípio variacional exige que $\vec{X}(\phi)$ tenha suporte compacto e portanto podemos, sem perda de generalidade, supor que $\vec{X}$ tenha suporte na base compacto, o último termo se anula pelo teorema de Stokes. Assim, obtemos

$$
S_{\mathcal{H}}^{\prime}[\phi] \cdot \vec{X}(\phi)=-\int_{M} \phi^{*}\left(i_{\vec{X}} \omega_{\mathcal{H}}\right)
$$

o que demonstra o teorema.

Uma outra fórmula explícita para o operador de De Donder-Weyl que pode ser obtida da equação (3.27) eliminando-se $\vec{X}$ é a seguinte:

$$
\begin{aligned}
\mathcal{D}_{\mathcal{H}}(\varphi, \pi, \partial \varphi, \partial \pi) \\
=-\left(\partial_{\mu} \varphi^{i}\left(\frac{\partial H}{\partial q^{i}}(\varphi, \pi)+\partial_{\nu} \pi_{i}^{\nu}\right)+\partial_{\mu} \pi_{i}^{\nu}\left(\frac{\partial H}{\partial p_{i}^{\nu}}(\varphi, \pi)-\partial_{\nu} \varphi^{i}\right)\right) d x^{\mu} \otimes d^{n} x \\
\quad+\left(\frac{\partial H}{\partial q^{i}}(\varphi, \pi)+\partial_{\mu} \pi_{i}^{\mu}\right) d q^{i} \otimes d^{n} x+\left(\frac{\partial H}{\partial p_{i}^{\mu}}(\varphi, \pi)-\partial_{\mu} \varphi^{i}\right) d p_{i}^{\mu} \otimes d^{n} x
\end{aligned}
$$


Passemos agora à apresentação dos nossos teoremas principais. Para tanto, continuamos trabalhando no âmbito hamiltoniano, e restringimo-nos a considerar funcionais $F$ associados a hipersuperfícies $\Sigma$, tipicamente hipersuperfícies de Cauchy, e formas de grau $n-1$ sobre $\vec{J}^{\circledast} E$ obtidas de formas localmente hamiltonianas de grau $n-1$ sobre $J^{\circledast} E$ por pull-back com alguma hamiltoniana $\mathcal{H}$. É importante lembrar que esta classe de formas é mais ampla do que a classe de formas localmente hamiltonianas de grau $n-1$ sobre $\vec{J}^{\circledast} E$, pois não está se exigindo que os correspondentes campos hamiltonianos sobre $J^{\oplus} E$ sejam tangentes à imagem de $\mathcal{H}$.

Teorema 3.2 Dada uma hamiltoniana $\mathcal{H}$, seja $f$ uma $(n-1)$-forma hamiltoniana sobre o espaço de multifase estendido $J^{\oplus E}$ tal que o campo hamiltoniano $X_{f}$ associado a $f$ seja projetável, conforme a Definição 1.7; denotaremos a sua projeção sobre $\vec{J}^{\circledR} E$ por $\vec{X}_{f}$ e a sua projeção sobre $M$ por $X_{f, M} \cdot{ }^{3}$ Por outro lado, seja $\Sigma$ uma hipersuperfície tipo espaço em $M$ sem bordo e $F$ o funcional sobre $\mathcal{S}$ associado a $\Sigma$ e $\mathcal{H}^{*} f$, conforme a Definição 3.1. Então o campo hamiltoniano funcional $X_{F}$ associado a $F$, definido pela condição (3.14), é dado pela seguinte fórmula explícita:

$$
X_{F}[\phi]=\vec{X}_{f}(\phi)-T \phi\left(X_{f, M}\right)
$$

Demonstração. Para mostrar que a expressão na equação (3.30) satisfaz a condição (3.14), para todo $\phi \in \mathcal{S}$ e todo $\delta \phi \in T_{\phi} \mathcal{S} \subset T_{\phi} \mathcal{C}$, começamos representando $\delta \phi$ como a composição de $\phi$ com algum campo vetorial vertical $\vec{X}$ sobre $\vec{J}{ }^{\circledast} E$, conforme a equação (3.21). Primeiro, calculamos o lado esquerdo da equação (3.14), inserindo as equações (3.5), (3.7) e (3.30):

$$
\Omega_{\phi}\left(X_{F}[\phi], \vec{X}(\phi)\right)=\int_{\Sigma} \phi^{*} \omega_{\mathcal{H}}\left(X_{F}[\phi], \vec{X}(\phi)\right)=\int_{\Sigma} \phi^{*} \omega_{\mathcal{H}}\left(\vec{X}_{f}(\phi)-T \phi\left(X_{f, M}\right), \vec{X}(\phi)\right) .
$$

Observemos agora que a integral

$$
\int_{\Sigma} \phi^{*} \omega_{\mathcal{H}}\left(T \phi\left(X_{f, M}\right), \vec{X}(\phi)\right)=-\int_{\Sigma} i_{X, M}\left(\phi^{*}\left(i_{\vec{X}} \omega_{\mathcal{H}}\right)\right)
$$

se anula, conforme o Teorema 3.1, pelo fato de $\phi$ ser uma solução das equações de movimento. Portanto,

$$
\Omega_{\phi}\left(X_{F}[\phi], \vec{X}(\phi)\right)=\int_{\Sigma} \phi^{*}\left(i_{\vec{X}} i_{\vec{X}_{f}} \omega_{\mathcal{H}}\right)
$$

\footnotetext{
${ }^{3}$ A existência destas projeções é automaticamente garantida exceto no caso $N=1$; veja a Definição 1.7 e a discussão subsequente.
} 
Por outro lado, segundo a Proposição 3.1, o lado direito da equação (3.14) vale

$$
\begin{aligned}
F^{\prime}[\phi] \cdot \vec{X}(\phi) & =\int_{\Sigma} \phi^{*}\left(L_{\vec{X}}\left(\mathcal{H}^{*} f\right)\right)=\int_{\Sigma} \phi^{*}\left(d i_{\vec{X}}\left(\mathcal{H}^{*} f\right)+i_{\vec{X}} d\left(\mathcal{H}^{*} f\right)\right) \\
& =\int_{\partial \Sigma} \phi^{*}\left(i_{\vec{X}}\left(\mathcal{H}^{*} f\right)\right)+\int_{\Sigma} \phi^{*}\left(i_{\vec{X}} \mathcal{H}^{*}\left(i_{X_{f}} \omega\right)\right) .
\end{aligned}
$$

O primeiro termo é zero, pois por hipótese, $\partial \Sigma=\emptyset$. e portanto, pela equação (1.170),

$$
F^{\prime}[\phi] \cdot \vec{X}(\phi)=\int_{\Sigma} \phi^{*}\left(i_{\vec{X}} i_{\vec{X}_{f}} \omega_{\mathcal{H}}+i_{\vec{X}} \mathcal{H}^{*}\left(i_{X_{f}^{\mathcal{H}}} \omega\right)\right)
$$

Agora, o segundo termo é zero pois, em relação à projeção de $\vec{J}{ }^{\circledast} E$ sobre $M$, o campo vetorial $\vec{X}_{g}^{V}$ é vertical enquanto que considerando o pull-back da equação (1.171) com $\mathcal{H}$ mostra que a $n$-forma $\mathcal{H}^{*}\left(i_{X_{j}} \boldsymbol{\psi} \omega\right)$ é horizontal.

De forma semelhante, podemos calcular explicitamente o colchete de Peierls - DeWitt entre dois funcionais associados a $(n-1)$-formas hamiltonianas:

Teorema 3.3 Dada uma hamiltoniana $\mathcal{H}$, sejam $f$ e $g(n-1)$-formas hamiltonianas sobre o espaço de multifase estendido $J^{\circledR} E$ tais que os campos hamiltonianos $X_{f}$ associados a $f$ e $X_{g}$ associados a $g$ sejam projetáveis, conforme a Definição 1.7; denotaremos as respectivas projeções sobre $\vec{J}^{\circledast} E$ por $\vec{X}_{f}$ e $\vec{X}_{g}$ e as respectivas projeçóes sobre $M$ por $X_{f, M}$ e $X_{g, M} \cdot{ }^{4}$ Por outro lado, seja $\Sigma$ uma hipersuperfície tipo espaço de $M$ sem bordo, e sejam $F$ e $G$ os funcionais sobre $\mathcal{S}$ associados a $\Sigma$ e às formas $\mathcal{H}^{*} f$ e $\mathcal{H}^{*} g$, respectivamente, conforme a Definição 3.1, i.e.,

$$
F[\phi]=\int_{\Sigma}(\mathcal{H} \circ \phi)^{*} f \quad, \quad G[\phi]=\int_{\Sigma}(\mathcal{H} \circ \phi)^{*} g .
$$

Então o colchete de Peierls - DeWitt entre $F$ e G é o funcional sobre $\mathcal{S}$ associado a $\Sigma$ e à forma $\mathcal{H}^{*}\{f, g\} \mathcal{H}$, i.e.,

$$
\{F, G\}[\phi]=\int_{\Sigma}(\mathcal{H} \circ \phi)^{*}\{f, g\}_{\mathcal{H}}
$$

onde $\{f, g\}_{\mathcal{H}}$ é um colchete de Poisson modificado entre $f$ e $g$ :

$$
\{f, g\}_{\mathcal{H}}=i_{X_{g}} i_{X_{f}} \omega-i_{X_{g}}{ }^{{ }_{X}^{\mathcal{H}}} \boldsymbol{} \omega-i_{X_{g}^{\mathcal{H}}} i_{X_{f}} \omega \text { modulo termos exatos . }
$$

Explicitamente,

$$
\{F, G\}[\phi]=\int_{\Sigma} \phi^{*}\left(i_{\vec{X}_{g}} i_{\vec{X}_{f}} \omega_{\mathcal{H}}\right)
$$

\footnotetext{
${ }^{4}$ A existência destas projeções é automaticamente garantida exceto no caso $N=1$; veja a Definição 1.7 e a discussão subsequente.
} 
DemonstraÇÃo. Representando $\vec{X}_{g}(\phi)-T \phi\left(X_{g, M}\right)$ como a composição de $\phi$ com algum campo vetorial vertical $\vec{X}_{g}^{V}$ sobre $\vec{J}^{\circledast} E$, conforme a equação (3.21), i.e.,

$$
\vec{X}_{g}^{V}(\phi)=\vec{X}_{g}(\phi)-T \phi\left(X_{g, M}\right)
$$

e usando as equações (3.18) e (3.22), vemos que o lado esquerdo da expressão (3.32) é dado por

$$
\begin{aligned}
\{F, G\}[\phi] & =F^{\prime}[\phi] \cdot X_{G}[\phi]=\int_{\Sigma} \phi^{*}\left(L_{\vec{X}_{g}^{V}}\left(\mathcal{H}^{*} f\right)\right)=\int_{\Sigma} \phi^{*}\left(d i_{\vec{X}_{g}^{V}}\left(\mathcal{H}^{*} f\right)+i_{\vec{X}_{g}^{V}} d\left(\mathcal{H}^{*} f\right)\right) \\
& =\int_{\partial \Sigma} \phi^{*}\left(i_{\vec{X}_{g}^{V}}\left(\mathcal{H}^{*} f\right)\right)+\int_{\Sigma} \phi^{*}\left(i_{\vec{X}_{g}^{V}}\left(\mathcal{H}^{*}\left(i_{X_{f}} \omega\right)\right)\right)
\end{aligned}
$$

O primeiro termo é zero pois por hipótese, $\partial \Sigma=\emptyset$, e portanto, pela equação $(1.170)$,

$$
\{F, G\}[\phi]=\int_{\Sigma} \phi^{*}\left(i_{\vec{X}_{g}^{V}} i_{\vec{X}_{f}} \omega_{\mathcal{H}}+i_{\vec{X}_{g}^{V}} \mathcal{H}^{*}\left(i_{X_{f}^{\mathcal{H}}} \omega\right)\right) \text {. }
$$

Agora, o segundo termo é zero pois, em relação à projeção de $\vec{J}{ }^{\circledast} E$ sobre $M$, o campo vetorial $\vec{X}_{g}^{V}$ é vertical enquanto que considerando o pull-back da equação (1.171) com $\mathcal{H}$ mostra que a $n$-forma $\mathcal{H}^{*}\left(i_{X_{f}^{\mathcal{H}} \omega}\right)$ é horizontal. Usando a relação $\vec{X}_{g}^{V}(\phi)=\vec{X}_{g}(\phi)-T \phi\left(X_{g, M}\right)$, obtemos

$$
\{F, G\}[\phi]=\int_{\Sigma} \phi^{*}\left(i_{\vec{X}_{g}} i_{\vec{X}_{f}} \omega_{\mathcal{H}}\right)-\int_{\Sigma} i_{X_{g, M}}\left(\phi^{*}\left(i_{\vec{X}_{f}} \omega_{\mathcal{H}}\right)\right) .
$$

Novamente, o segundo termo se anula, desta vez devido ao fato de $\phi$ ser uma solução das equações de movimento, conforme o Teorema 3.1. Assim, chegamos à equação (3.34). Finalmente, observamos que

$$
i_{\vec{X}_{g}} i_{\vec{X}_{f}} \omega_{\mathcal{H}}=\mathcal{H}^{*}\left(i_{X_{g}} i_{X_{f}} \omega+i_{X_{g}} i_{X_{f}^{\mathcal{H}}} \omega-i_{X_{g}^{\mathcal{H}}} i_{X_{f}} \omega\right)
$$

já que o termo $i_{X_{g}^{\mathcal{H}}{ }^{i}{ }_{X_{f}} \omega} \omega$ se anula automaticamente.

Resta, como tarefa para o futuro, determinar os termos exatos que faltam na equação (3.33) para garantir que este colchete modificado satisfaça a identidade de Jacobi, em analogia ao Teorema 1.7. 



\section{Referenciais não holônomos}

\section{A.1 Derivadas covariantes no fibrado vertical}

No que segue, apresentaremos uma demonstração independente do Teorema 2.1, que por conveniência reproduzimos aqui:

Teorema A.1 Dada uma conexão $\Gamma_{E}$ em E sobre $M$ e uma conexão linear parcial $\tilde{\Gamma}_{V E}^{V E}$ em $V E$ sobre $E$ ao longo de $V E$, existe uma única extensão de $\tilde{\Gamma}_{V E}^{V E}$ para uma conexão linear total $\tilde{\Gamma}_{V E}$ em VE sobre E cuja composição com $\Gamma_{E}$ é a conexão vertical $\hat{\Gamma}_{V E}$ em VE sobre $M$ induzida por $\Gamma_{E}$. Em termos de derivadas covariantes de seções $X$ de $V E$, isto é, campos vetoriais verticais $X$ sobre $E$, temos

$$
\nabla_{i} X^{j}=\partial_{i} X^{j}+\Gamma_{i r}^{j} X^{r}
$$

$e$

$$
\nabla_{\mu} X^{j}=\partial_{\mu} X^{j}-\left(\partial_{i} \Gamma_{\mu}^{j}+\Gamma_{r i}^{j} \Gamma_{\mu}^{r}\right) X^{i}
$$

ou, equivalentemente,

$$
\nabla_{\Gamma\left(\partial_{\mu}\right)} X^{j}=\partial_{\mu} X^{j}+\Gamma_{\mu}^{i} \partial_{i} X^{j}-\partial_{i} \Gamma_{\mu}^{j} X^{i}
$$

Demonstração. Para checar que estas expressões realmente definem uma derivada covariante de seções $X$ de $V E$, faremos uma mudança de coordenadas, do antigo sistema de coordenadas locais $\left(x^{\mu}, q^{i} ; \dot{q}^{i}\right)$ de $V E$ para um novo sistema de coordenadas locais $\left(x^{\prime \kappa}, q^{\prime k} ; \dot{q}^{\prime k}\right)$ conforme a equação (1.20), que implica

$$
\dot{q}^{\prime k}=\frac{\partial q^{\prime k}}{\partial q^{i}} \dot{q}^{i} .
$$


Conforme a equação (A.21), os campos de coordenadas $\partial_{i}$ sobre $E$ se transformam de maneira covariante, enquanto que os campos de coordenadas $\partial_{\mu}$ sobre $E$ não. No entanto, combinando as equações (A.19), (A.21) e (2.14), concluímos que os levantamentos horizontais dos campos de coordenadas $\partial_{\mu}$ sobre $M$ para $E$, estes sim, se transformam de maneira covariante. Assim, temos

$$
\partial_{k}^{\prime}=\frac{\partial q^{i}}{\partial q^{\prime k}} \partial_{i} \quad, \quad \Gamma\left(\partial_{\kappa}^{\prime}\right)=\frac{\partial x^{\mu}}{\partial x^{\prime \kappa}} \Gamma\left(\partial_{\mu}\right)
$$

A lei de transformação para as componentes de seções $X$ de $V E$ é dada pela equação (A.4), e a lei de transformação para as suas derivadas parciais pode ser calculada a partir desta e das equações (A.21):

$$
\begin{aligned}
\partial_{i} X^{j} \longrightarrow \partial_{k}^{\prime} X^{\prime l}= & \frac{\partial q^{i}}{\partial q^{\prime k}} \frac{\partial q^{\prime l}}{\partial q^{j}} \partial_{i} X^{j}+\frac{\partial q^{i}}{\partial q^{\prime k}} \frac{\partial^{2} q^{\prime l}}{\partial q^{i} \partial q^{j}} X^{j} \\
\partial_{\mu} X^{j} \longrightarrow \partial_{\kappa}^{\prime} X^{\prime l}= & \frac{\partial x^{\mu}}{\partial x^{\prime \kappa}} \frac{\partial q^{\prime l}}{\partial q^{j}} \partial_{\mu} X^{j}+\frac{\partial x^{\mu}}{\partial x^{\prime \kappa}} \frac{\partial^{2} q^{\prime l}}{\partial x^{\mu} \partial q^{j}} X^{j} \\
& -\frac{\partial x^{\mu}}{\partial x^{\prime \kappa}} \frac{\partial q^{\prime r}}{\partial x^{\mu}} \frac{\partial q^{i}}{\partial q^{\prime r}} \frac{\partial q^{\prime l}}{\partial q^{j}} \partial_{i} X^{j}-\frac{\partial x^{\mu}}{\partial x^{\prime \kappa}} \frac{\partial q^{\prime r}}{\partial x^{\mu}} \frac{\partial q^{i}}{\partial q^{\prime r}} \frac{\partial^{2} q^{\prime l}}{\partial q^{i} \partial q^{j}} X^{j}
\end{aligned}
$$

Portanto, a lei de transformação (A.26) mostra que as derivadas covariantes ao longo dos campos de coordenadas $\partial_{i}$ sobre $E$, que aparecem na equação (A.1), se transformam de maneira covariante:

$$
\begin{aligned}
\nabla_{k}^{\prime} X^{\prime l}= & \partial_{k}^{\prime} X^{\prime l}+\Gamma_{k s}^{\prime l} X^{\prime s} \\
= & \frac{\partial q^{i}}{\partial q^{\prime k}} \frac{\partial q^{\prime l}}{\partial q^{j}} \partial_{i} X^{j}+\frac{\partial q^{i}}{\partial q^{\prime k}} \frac{\partial^{2} q^{\prime l}}{\partial q^{i} \partial q^{j}} X^{j} \\
& \quad+\left(\frac{\partial q^{\prime l}}{\partial q^{j}} \frac{\partial q^{i}}{\partial q^{\prime k}} \frac{\partial q^{r}}{\partial q^{\prime s}} \Gamma_{i r}^{j}-\frac{\partial^{2} q^{\prime l}}{\partial q^{i} \partial q^{r}} \frac{\partial q^{i}}{\partial q^{\prime k}} \frac{\partial q^{r}}{\partial q^{\prime s}}\right)\left(\frac{\partial q^{\prime s}}{\partial q^{t}} X^{t}\right) \\
= & \frac{\partial q^{i}}{\partial q^{\prime k}} \frac{\partial q^{\prime l}}{\partial q^{j}} \nabla_{i} X^{j}
\end{aligned}
$$

Por outro lado, a lei de transformação (A.21) mostra que as derivadas covariantes ao longo dos campos de coordenadas $\partial_{\mu}$ sobre $E$, que aparecem na equação (A.2), não se transformam de maneira covariante, o que não deve surpreender tende em vista que os próprios campos $\partial_{\mu}$ sobre $E$ não se transformam de maneira covariante. Covariância só pode ser estabelecida para as derivadas covariantes ao longo dos campos $\Gamma\left(\partial_{\mu}\right)$ sobre $E$ obtidos a partir dos campos de coordenadas $\partial_{\mu}$ sobre $M$ por levantamento horizontal, pois estes sim se transformam de 
maneira covariante (veja a equação (A.5)). De fato, temos

$$
\begin{aligned}
\nabla_{\Gamma\left(\partial_{\kappa}^{\prime}\right) X^{\prime l}=} & \partial_{\kappa}^{\prime} X^{\prime l}+\Gamma_{\kappa}^{\prime k} \partial_{k}^{\prime} X^{\prime l}-\partial_{k}^{\prime} \Gamma_{\kappa}^{\prime l} X^{\prime k} \\
= & \frac{\partial x^{\mu}}{\partial x^{\prime \kappa}} \frac{\partial q^{\prime l}}{\partial q^{j}} \partial_{\mu} X^{j}+\frac{\partial x^{\mu}}{\partial x^{\prime \kappa}} \frac{\partial^{2} q^{\prime l}}{\partial x^{\mu} \partial q^{j}} X^{j} \\
& -\frac{\partial x^{\mu}}{\partial x^{\prime \kappa}} \frac{\partial q^{\prime r}}{\partial x^{\mu}} \frac{\partial q^{i}}{\partial q^{\prime r}} \frac{\partial q^{\prime l}}{\partial q^{j}} \partial_{i} X^{j}-\frac{\partial x^{\mu}}{\partial x^{\prime \kappa}} \frac{\partial q^{\prime r}}{\partial x^{\mu}} \frac{\partial q^{i}}{\partial q^{\prime r}} \frac{\partial^{2} q^{\prime \prime}}{\partial q^{i} \partial q^{j}} X^{j} \\
& +\left(\frac{\partial x^{\mu}}{\partial x^{\prime \kappa}} \frac{\partial q^{\prime k}}{\partial q^{i}} \Gamma_{\mu}^{i}+\frac{\partial x^{\mu}}{\partial x^{\prime \kappa}} \frac{\partial q^{\prime k}}{\partial x^{\mu}}\right)\left(\frac{\partial q^{r}}{\partial q^{\prime k}} \frac{\partial q^{\prime l}}{\partial q^{j}} \partial_{r} X^{j}+\frac{\partial q^{r}}{\partial q^{\prime k}} \frac{\partial^{2} q^{\prime l}}{\partial q^{r} \partial q^{j}} X^{j}\right) \\
& -\frac{\partial x^{\mu}}{\partial x^{\prime \kappa}} \frac{\partial q^{i}}{\partial q^{\prime k}}\left(\frac{\partial q^{\prime l}}{\partial q^{j}} \partial_{i} \Gamma_{\mu}^{j}+\frac{\partial^{2} q^{\prime l}}{\partial q^{i} \partial q^{j}} \Gamma_{\mu}^{j}+\frac{\partial^{2} q^{\prime l}}{\partial x^{\mu} \partial q^{i}}\right)\left(\frac{\partial q^{\prime k}}{\partial q^{r}} X^{r}\right) \\
= & \frac{\partial x^{\mu}}{\partial x^{\prime \kappa}} \frac{\partial q^{\prime l}}{\partial q^{j}} \nabla_{\Gamma\left(\partial_{\mu}\right)^{\prime}} X^{j}+\frac{\partial x^{\mu}}{\partial x^{\prime \kappa}} \frac{\partial^{2} q^{\prime l}}{\partial x^{\mu} \partial q^{j}} X^{j}-\frac{\partial x^{\mu}}{\partial x^{\prime \kappa}} \frac{\partial q^{\prime r}}{\partial x^{\mu}} \frac{\partial q^{i}}{\partial q^{\prime r}} \frac{\partial q^{\prime l}}{\partial q^{j}} \partial_{i} X^{j} \\
& -\frac{\partial x^{\mu}}{\partial x^{\prime \kappa}} \frac{\partial q^{\prime r}}{\partial x^{\mu}} \frac{\partial q^{i}}{\partial q^{\prime r}} \frac{\partial^{2} q^{\prime l}}{\partial q^{i} \partial q^{j}} X^{j}+\frac{\partial x^{\mu}}{\partial x^{\prime \kappa}} \frac{\partial^{2} q^{\prime l}}{\partial q^{i} \partial q^{j}} \Gamma_{\mu}^{i} X^{j} \\
& +\frac{\partial x^{\mu}}{\partial x^{\prime \kappa}} \frac{\partial q^{\prime k}}{\partial x^{\mu}} \frac{\partial q^{r}}{\partial q^{\prime k}} \frac{\partial q^{\prime l}}{\partial q^{j}} \partial_{r} X^{j}+\frac{\partial x^{\mu}}{\partial x^{\prime \kappa}} \frac{\partial q^{\prime k}}{\partial x^{\mu}} \frac{\partial q^{r}}{\partial q^{\prime k}} \frac{\partial^{2} q^{\prime l}}{\partial q^{r} \partial q^{j}} X^{j} \\
& -\frac{\partial x^{\mu}}{\partial x^{\prime \kappa}}\left(\frac{\partial^{2} q^{\prime l}}{\partial q^{i} \partial q^{j}} \Gamma_{\mu}^{j}+\frac{\partial^{2} q^{\prime l}}{\partial x^{\mu} \partial q^{i}}\right) X^{i} \\
= & \frac{\partial x^{\mu}}{\partial x^{\prime \kappa}} \frac{\partial q^{\prime l}}{\partial q^{j}} \nabla_{\Gamma\left(\partial_{\mu}\right)} X^{j} \cdot
\end{aligned}
$$

Isto implica na lei de transformação desejada:

$$
\begin{aligned}
\nabla_{i} X^{j} & \longrightarrow \nabla_{k} X^{\prime l}=\frac{\partial q^{i}}{\partial q^{\prime k}} \frac{\partial q^{\prime l}}{\partial q^{j}} \nabla_{i} X^{j} \\
\nabla_{\Gamma\left(\partial_{\mu}\right)} X^{j} & \longrightarrow \nabla_{\Gamma\left(\partial_{\kappa}^{\prime}\right)} X^{\prime l}=\frac{\partial x^{\mu}}{\partial x^{\prime \kappa}} \frac{\partial q^{\prime l}}{\partial q^{j}} \nabla_{\Gamma\left(\partial_{\mu}\right)} X^{j}
\end{aligned}
$$




\section{A.2 Referenciais não holônomos no fibrado dos cojatos}

Teorema A.2 Dadas uma conexão $\Gamma_{E}$ em $E$ sobre $M$, uma conexão linear parcial $\Gamma_{V E}^{V E}$ em VE sobre $E$ ao longo de $V E$ e uma conexão linear $\Gamma_{M}$ em $M$, os referenciais de Darboux associados a este conjunto de conexóes se transformam de maneira covariante sob transformações de coordenadas locais adaptadas em $J^{\otimes} E$, como especificado pelas equações (2.144) e (2.145).

DEMONSTRAÇÃo. Restringiremo-nos a provar a lei de transformação (2.144) para os referenciais de Darboux no fibrado tangente de $J^{\otimes} E$, definidos pelas equações (2.135)-(2.138), sendo que a definição dos referenciais de Darboux no fibrado cotangente de $J^{\oplus} E$, dada pelas equações (2.139)-(2.142), e a lei de transformação (2.145) para estes é obtida por dualização. Começamos por reproduzir as equações (2.135)-(2.138):

$$
\begin{gathered}
e_{0}=\frac{\partial}{\partial p}, \\
e_{\mu}^{i}=\frac{\partial}{\partial p_{i}^{\mu}}-\Gamma_{\mu}^{i} \frac{\partial}{\partial p}, \\
e_{i}=\frac{\partial}{\partial q^{i}}+\Gamma_{i j}^{r} p_{r}^{\nu} \frac{\partial}{\partial p_{j}^{\nu}}-\left(\partial_{i} \Gamma_{\nu}^{r}+\Gamma_{i j}^{r} \Gamma_{\nu}^{j}\right) p_{r}^{\nu} \frac{\partial}{\partial p}, \\
e_{\mu}=\frac{\partial}{\partial x^{\mu}}+\Gamma_{\mu}^{j} \frac{\partial}{\partial q^{j}}-\left(\partial_{j} \Gamma_{\mu}^{r} p_{r}^{\nu}+\Gamma_{\mu \rho}^{\nu} p_{j}^{\rho}-\Gamma_{\mu \rho}^{\rho} p_{j}^{\nu}\right) \frac{\partial}{\partial p_{j}^{\nu}} \\
-\left(\partial_{\mu} \Gamma_{\nu}^{j} p_{j}^{\nu}+\Gamma_{\mu}^{i} \partial_{i} \Gamma_{\nu}^{r} p_{r}^{\nu}-\Gamma_{\nu}^{j} \partial_{j} \Gamma_{\mu}^{r} p_{r}^{\nu}-\Gamma_{\mu \rho}^{\nu} \Gamma_{\nu}^{j} p_{j}^{\rho}-\Gamma_{\mu \rho}^{\rho} p\right) \frac{\partial}{\partial p} .
\end{gathered}
$$

Para mostrarmos que estas expressões realmente definem um referencial covariante, faremos uma mudança de coordenadas, do antigo sistema de coordenadas locais $\left(x^{\mu}, q^{i}, p_{i}^{\mu}, p\right)$ de $J^{\oplus} E$ para o novo sistema de coordenadas locais $\left(x^{\prime \kappa}, q^{\prime k}, p_{k}^{\prime \kappa}, p^{\prime}\right)$, de acordo com

$$
p_{k}^{\prime \kappa}=p_{k}^{\prime \kappa}\left(x^{\mu}, q^{i}, p_{i}^{\mu}\right), p^{\prime}=p^{\prime}\left(x^{\mu}, q^{i}, p_{i}^{\mu}, p\right),
$$

onde

$$
p_{k}^{\prime \kappa}=\operatorname{det}\left(\frac{\partial x}{\partial x^{\prime}}\right) \frac{\partial x^{\prime \kappa}}{\partial x^{\mu}} \frac{\partial q^{i}}{\partial q^{\prime k}} p_{i}^{\mu} \quad, \quad p^{\prime}=\operatorname{det}\left(\frac{\partial x}{\partial x^{\prime}}\right)\left(p-\frac{\partial q^{\prime k}}{\partial x^{\mu}} \frac{\partial q^{i}}{\partial q^{\prime k}} p_{i}^{\mu}\right) .
$$

A correspondente lei de transformação para o referencial pode ser determinado diferenciando a última equação, obtendo a lei de transformação para os diferenciais:

$$
\begin{aligned}
d p_{k}^{\prime \kappa}= & \frac{\partial}{\partial x^{\mu}}\left(\operatorname{det}\left(\frac{\partial x}{\partial x^{\prime}}\right) \frac{\partial x^{\prime \kappa}}{\partial x^{\nu}} \frac{\partial q^{j}}{\partial q^{\prime k}}\right) p_{j}^{\nu} d x^{\mu}+\operatorname{det}\left(\frac{\partial x}{\partial x^{\prime}}\right) \frac{\partial x^{\prime \kappa}}{\partial x^{\prime \prime}} \frac{\partial}{\partial q^{i}}\left(\frac{\partial q^{j}}{\partial q^{\prime k}}\right) p_{j}^{\nu} d q^{i} \\
& +\operatorname{det}\left(\frac{\partial x}{\partial x^{\prime}}\right) \frac{\partial x^{\prime \kappa}}{\partial x^{\mu}} \frac{\partial q^{i}}{\partial q^{\prime k}} d p_{i}^{\mu}
\end{aligned}
$$




$$
\begin{aligned}
d p^{\prime}= & \frac{\partial}{\partial x^{\mu}}\left(\operatorname{det}\left(\frac{\partial x}{\partial x^{\prime}}\right)\left(p-\frac{\partial q^{\prime k}}{\partial x^{\nu}} \frac{\partial q^{j}}{\partial q^{\prime k}} p_{j}^{\nu}\right)\right) d x^{\mu}-\operatorname{det}\left(\frac{\partial x}{\partial x^{\prime}}\right) \frac{\partial}{\partial q^{i}}\left(\frac{\partial q^{\prime k}}{\partial x^{\nu}} \frac{\partial q^{j}}{\partial q^{\prime k}}\right) p_{j}^{\nu} d q^{i} \\
& -\operatorname{det}\left(\frac{\partial x}{\partial x^{\prime}}\right) \frac{\partial q^{\prime k}}{\partial x^{\mu}} \frac{\partial q^{i}}{\partial q^{\prime k}} d p_{i}^{\mu}+\operatorname{det}\left(\frac{\partial x}{\partial x^{\prime}}\right) d p .
\end{aligned}
$$

Usando as relações

$$
\begin{gathered}
\partial_{\alpha}\left(A^{-1}\right)=-A^{-1} \partial_{\alpha} A A^{-1}, \\
\partial_{\alpha} \operatorname{det}(A)=\operatorname{det}(A) \operatorname{tr}\left(A^{-1} \partial_{\alpha} A\right),
\end{gathered}
$$

para funções a valores matriciais $A$ das variáveis $z^{\alpha}$, obtemos

$$
\begin{aligned}
& d x^{\prime \kappa}=\frac{\partial x^{\prime \kappa}}{\partial x^{\mu}} d x^{\mu} \\
& d q^{\prime k}=\frac{\partial q^{\prime k}}{\partial x^{\mu}} d x^{\mu}+\frac{\partial q^{\prime k}}{\partial q^{i}} d q^{i} \\
& d p_{k}^{\prime \kappa}=\operatorname{det}\left(\frac{\partial x}{\partial x^{\prime}}\right)\left(\left(-\frac{\partial^{2} x^{\prime \sigma}}{\partial x^{\mu} \partial x^{\rho}} \frac{\partial x^{\rho}}{\partial x^{\prime \sigma}} \frac{\partial x^{\prime \kappa}}{\partial x^{\nu}} \frac{\partial q^{j}}{\partial q^{\prime k}} p_{j}^{\nu}+\frac{\partial^{2} x^{\prime \kappa}}{\partial x^{\mu} \partial x^{\nu}} \frac{\partial q^{j}}{\partial q^{\prime k}} p_{j}^{\nu}\right.\right. \\
& \left.-\frac{\partial x^{\prime \kappa}}{\partial x^{\nu}} \frac{\partial q^{j}}{\partial q^{\prime s}} \frac{\partial^{2} q^{\prime s}}{\partial x^{\mu} \partial q^{r}} \frac{\partial q^{r}}{\partial q^{\prime k}} p_{j}^{\nu}\right) d x^{\mu} \\
& \left.-\frac{\partial x^{\prime \kappa}}{\partial x^{\nu}} \frac{\partial q^{j}}{\partial q^{\prime s}} \frac{\partial^{2} q^{\prime s}}{\partial q^{i} \partial q^{r}} \frac{\partial q^{r}}{\partial q^{\prime k}} p_{j}^{\nu} d q^{i}+\frac{\partial x^{\prime \kappa}}{\partial x^{\mu}} \frac{\partial q^{i}}{\partial q^{\prime k}} d p_{i}^{\mu}\right) \\
& d p^{\prime}=\operatorname{det}\left(\frac{\partial x}{\partial x^{\prime}}\right)\left(\left(\frac{\partial^{2} x^{\prime \sigma}}{\partial x^{\mu} \partial x^{\rho}} \frac{\partial x^{\rho}}{\partial x^{\prime \sigma}}\left(\frac{\partial q^{\prime k}}{\partial x^{\nu}} \frac{\partial q^{j}}{\partial q^{\prime k}} p_{j}^{\nu}-p\right)\right.\right. \\
& \left.-\frac{\partial^{2} q^{\prime k}}{\partial x^{\mu} \partial x^{\nu}} \frac{\partial q^{j}}{\partial q^{\prime k}} p_{j}^{\nu}+\frac{\partial q^{\prime k}}{\partial x^{\nu}} \frac{\partial q^{j}}{\partial q^{\prime s}} \frac{\partial^{2} q^{\prime s}}{\partial x^{\mu} \partial q^{r}} \frac{\partial q^{r}}{\partial q^{\prime k}} p_{j}^{\nu}\right) d x^{\mu} \\
& -\left(\frac{\partial^{2} q^{\prime k}}{\partial x^{\nu} \partial q^{i}} \frac{\partial q^{j}}{\partial q^{\prime k}} p_{j}^{\nu}-\frac{\partial q^{\prime k}}{\partial x^{\nu}} \frac{\partial q^{j}}{\partial q^{\prime s}} \frac{\partial^{2} q^{\prime s}}{\partial q^{i} \partial q^{r}} \frac{\partial q^{r}}{\partial q^{\prime k}} p_{j}^{\nu}\right) d q^{i} \\
& \left.-\frac{\partial q^{\prime k}}{\partial x^{\mu}} \frac{\partial q^{i}}{\partial q^{\prime k}} d p_{i}^{\mu}+d p\right) \text {. }
\end{aligned}
$$

Dualizando, obtemos o resultado

$$
\begin{gathered}
\frac{\partial}{\partial p^{\prime}}=\operatorname{det}\left(\frac{\partial x^{\prime}}{\partial x}\right) \frac{\partial}{\partial p} \\
\frac{\partial}{\partial p_{k}^{\prime \kappa}}=\operatorname{det}\left(\frac{\partial x^{\prime}}{\partial x}\right)\left(\frac{\partial x^{\mu}}{\partial x^{\prime \kappa}} \frac{\partial q^{\prime k}}{\partial q^{i}} \frac{\partial}{\partial p_{i}^{\mu}}+\frac{\partial x^{\mu}}{\partial x^{\prime \kappa}} \frac{\partial q^{\prime k}}{\partial x^{\mu}} \frac{\partial}{\partial p}\right)
\end{gathered}
$$




$$
\begin{aligned}
\frac{\partial}{\partial q^{\prime k}}= & \frac{\partial q^{i}}{\partial q^{\prime k}} \frac{\partial}{\partial q^{i}}+\frac{\partial^{2} q^{\prime s}}{\partial q^{i} \partial q^{r}} \frac{\partial q^{j}}{\partial q^{\prime s}} \frac{\partial q^{r}}{\partial q^{\prime k}} p_{j}^{\mu} \frac{\partial}{\partial p_{i}^{\mu}}+\frac{\partial^{2} q^{\prime s}}{\partial x^{\mu} \partial q^{r}} \frac{\partial q^{j}}{\partial q^{\prime s}} \frac{\partial q^{r}}{\partial q^{\prime k}} p_{j}^{\mu} \frac{\partial}{\partial p} \\
\frac{\partial}{\partial x^{\prime \kappa}}= & \frac{\partial x^{\mu}}{\partial x^{\prime \kappa}} \frac{\partial}{\partial x^{\mu}}-\frac{\partial x^{\mu}}{\partial x^{\prime \kappa}} \frac{\partial q^{\prime k}}{\partial x^{\mu}} \frac{\partial q^{i}}{\partial q^{\prime k}} \frac{\partial}{\partial q^{i}} \\
& +\left(\frac{\partial^{2} x^{\prime \sigma}}{\partial x^{\nu} \partial x^{\rho}} \frac{\partial x^{\rho}}{\partial x^{\prime \sigma}} \frac{\partial x^{\nu}}{\partial x^{\prime \kappa}} p_{i}^{\mu}-\frac{\partial^{2} x^{\prime \sigma}}{\partial x^{\nu} \partial x^{\rho}} \frac{\partial x^{\mu}}{\partial x^{\prime \sigma}} \frac{\partial x^{\rho}}{\partial x^{\prime \kappa}} p_{i}^{\nu}\right. \\
& \left.+\frac{\partial x^{\nu}}{\partial x^{\prime \kappa}} \frac{\partial^{2} q^{\prime k}}{\partial x^{\nu} \partial q^{i}} \frac{\partial q^{j}}{\partial q^{\prime k}} p_{j}^{\mu}-\frac{\partial x^{\nu}}{\partial x^{\prime \kappa}} \frac{\partial q^{\prime k}}{\partial x^{\nu}} \frac{\partial q^{r}}{\partial q^{\prime k}} \frac{\partial^{2} q^{\prime l}}{\partial q^{i} \partial q^{r}} \frac{\partial q^{j}}{\partial q^{\prime l}} p_{j}^{\mu}\right) \frac{\partial}{\partial p_{i}^{\mu}} \\
& +\frac{\partial x^{\mu}}{\partial x^{\prime \kappa}}\left(\frac{\partial^{2} q^{\prime k}}{\partial x^{\mu} \partial x^{\nu}} \frac{\partial q^{i}}{\partial q^{\prime k}} p_{i}^{\nu}-\frac{\partial q^{\prime \prime}}{\partial x^{\mu}} \frac{\partial q^{r}}{\partial q^{\prime l}} \frac{\partial^{2} q^{\prime k}}{\partial x^{\nu} \partial q^{r}} \frac{\partial q^{i}}{\partial q^{\prime k}} p_{i}^{\nu}\right. \\
& \left.\quad \frac{\partial^{2} x^{\prime \sigma}}{\partial x^{\mu} \partial x^{\nu}} \frac{\partial x^{\rho}}{\partial x^{\prime \sigma}} \frac{\partial q^{\prime k}}{\partial x^{\rho}} \frac{\partial q^{i}}{\partial q^{\prime k}} p_{i}^{\nu}+\frac{\partial^{2} x^{\prime \sigma}}{\partial x^{\mu} \partial x^{\rho}} \frac{\partial x^{\rho}}{\partial x^{\prime \sigma}} p\right) \frac{\partial}{\partial p}
\end{aligned}
$$

Para uma conexão em $E$, temos a lei de transformação

$$
\Gamma_{\mu}^{i} \longrightarrow \Gamma_{\kappa}^{\prime k}=\frac{\partial x^{\mu}}{\partial x^{\prime \kappa}} \frac{\partial q^{\prime k}}{\partial q^{i}} \Gamma_{\mu}^{i}+\frac{\partial x^{\mu}}{\partial x^{\prime \kappa}} \frac{\partial q^{\prime k}}{\partial x^{\mu}}
$$

que, como notaremos para uso posterior, implica

$$
p+\Gamma_{\mu}^{i} p_{i}^{\mu} \longrightarrow p^{\prime}+\Gamma_{\kappa}^{\prime k} p_{k}^{\prime \kappa}=\operatorname{det}\left(\frac{\partial x}{\partial x^{\prime}}\right)\left(p+\Gamma_{\mu}^{i} p_{i}^{\mu}\right)
$$

Para calcular a lei de transformação para as suas derivadas, usamos que, localmente em $E$,

$$
d x^{\prime \kappa}=\frac{\partial x^{\prime \kappa}}{\partial x^{\mu}} d x^{\mu} \quad, \quad d q^{\prime k}=\frac{\partial q^{\prime k}}{\partial x^{\mu}} d x^{\mu}+\frac{\partial q^{\prime k}}{\partial q^{i}} d q^{i}
$$

e dualizando

$$
\frac{\partial}{\partial x^{\prime \kappa}}=\frac{\partial x^{\mu}}{\partial x^{\prime \kappa}} \frac{\partial}{\partial x^{\mu}}-\frac{\partial x^{\mu}}{\partial x^{\prime \kappa}} \frac{\partial q^{\prime k}}{\partial x^{\mu}} \frac{\partial q^{i}}{\partial q^{\prime k}} \frac{\partial}{\partial q^{i}} \quad, \quad \frac{\partial}{\partial q^{\prime k}}=\frac{\partial q^{i}}{\partial q^{\prime k}} \frac{\partial}{\partial q^{i}}
$$

obtemos assim as leis de transformações procuradas

$$
\partial_{j} \Gamma_{\mu}^{i} \longrightarrow \partial_{l}^{\prime} \Gamma_{\kappa}^{\prime k}, \partial_{\nu} \Gamma_{\mu}^{i} \longrightarrow \partial_{\lambda}^{\prime} \Gamma_{\kappa}^{\prime k}
$$

onde

$$
\partial_{l}^{\prime} \Gamma_{\kappa}^{k}=\frac{\partial q^{j}}{\partial q^{\prime l}} \frac{\partial}{\partial q^{j}}\left(\frac{\partial x^{\mu}}{\partial x^{\prime \kappa}} \frac{\partial q^{\prime k}}{\partial q^{i}} \Gamma_{\mu}^{i}+\frac{\partial x^{\mu}}{\partial x^{\prime \kappa}} \frac{\partial q^{\prime k}}{\partial x^{\mu}}\right)
$$


e

ou explicitamente

$$
\partial_{\lambda}^{\prime} \Gamma_{\kappa}^{\prime k}=\frac{\partial x^{\nu}}{\partial x^{\prime \lambda}}\left(\frac{\partial}{\partial x^{\nu}}-\frac{\partial q^{\prime l}}{\partial x^{\nu}} \frac{\partial q^{j}}{\partial q^{\prime l}} \frac{\partial}{\partial q^{j}}\right)\left(\frac{\partial x^{\mu}}{\partial x^{\prime \kappa}} \frac{\partial q^{\prime k}}{\partial q^{i}} \Gamma_{\mu}^{i}+\frac{\partial x^{\mu}}{\partial x^{\prime \kappa}} \frac{\partial q^{\prime k}}{\partial x^{\mu}}\right)
$$

$$
\begin{gathered}
\partial_{l}^{\prime} \Gamma_{\kappa}^{\prime k}=\frac{\partial x^{\mu}}{\partial x^{\prime \kappa}} \frac{\partial q^{j}}{\partial q^{\prime l}}\left(\frac{\partial q^{\prime k}}{\partial q^{i}} \partial_{j} \Gamma_{\mu}^{i}+\frac{\partial^{2} q^{\prime k}}{\partial q^{j} \partial q^{i}} \Gamma_{\mu}^{i}+\frac{\partial^{2} q^{\prime k}}{\partial x^{\mu} \partial q^{j}}\right) \\
\partial_{\lambda}^{\prime} \Gamma_{\kappa}^{\prime k}=\frac{\partial x^{\mu}}{\partial x^{\prime \kappa}} \frac{\partial x^{\nu}}{\partial x^{\prime \lambda}}\left(\frac{\partial q^{\prime k}}{\partial q^{i}} \partial_{\nu} \Gamma_{\mu}^{i}+\frac{\partial^{2} q^{\prime k}}{\partial x^{\nu} \partial q^{i}} \Gamma_{\mu}^{i}-\frac{\partial^{2} x^{\prime \sigma}}{\partial x^{\nu} \partial x^{\mu}} \frac{\partial x^{\rho}}{\partial x^{\prime \sigma}} \frac{\partial q^{\prime k}}{\partial q^{i}} \Gamma_{\rho}^{i}\right. \\
+\frac{\partial^{2} q^{\prime k}}{\partial x^{\nu} \partial x^{\mu}}-\frac{\partial^{2} x^{\prime \sigma}}{\partial x^{\nu} \partial x^{\mu}} \frac{\partial x^{\rho}}{\partial x^{\prime \sigma}} \frac{\partial q^{\prime k}}{\partial x^{\rho}} \\
\left.-\frac{\partial q^{\prime l}}{\partial x^{\nu}} \frac{\partial q^{j}}{\partial q^{\prime l}}\left(\frac{\partial q^{\prime k}}{\partial q^{i}} \partial_{j} \Gamma_{\mu}^{i}+\frac{\partial^{2} q^{\prime k}}{\partial q^{j} \partial q^{i}} \Gamma_{\mu}^{i}+\frac{\partial^{2} q^{\prime k}}{\partial x^{\mu} \partial q^{j}}\right)\right) .
\end{gathered}
$$

Finalmente, para uma conexão linear em $M$ (isto é, no fibrado tangente $T M$ de $M$ )

e para uma conexão linear no fibrado vertical $V E$ de $E$

$$
\Gamma_{\mu \nu}^{\rho} \longrightarrow \Gamma_{\kappa \lambda}^{\prime \sigma}=\frac{\partial x^{\prime \sigma}}{\partial x^{\rho}} \frac{\partial x^{\mu}}{\partial x^{\prime \kappa}} \frac{\partial x^{\nu}}{\partial x^{\prime \lambda}} \Gamma_{\mu \nu}^{\rho}-\frac{\partial^{2} x^{\prime \sigma}}{\partial x^{\mu} \partial x^{\nu}} \frac{\partial x^{\mu}}{\partial x^{\prime \kappa}} \frac{\partial x^{\nu}}{\partial x^{\prime \lambda}},
$$

Isto implica que

$$
\Gamma_{i j}^{r} \longrightarrow \Gamma_{k l}^{s s}=\frac{\partial q^{\prime s}}{\partial q^{r}} \frac{\partial q^{i}}{\partial q^{\prime k}} \frac{\partial q^{j}}{\partial q^{\prime l}} \Gamma_{i j}^{r}-\frac{\partial^{2} q^{\prime s}}{\partial q^{i} \partial q^{j}} \frac{\partial q^{i}}{\partial q^{\prime k}} \frac{\partial q^{j}}{\partial q^{\prime l}} .
$$

$$
\partial_{j} \Gamma_{\mu}^{i}+\Gamma_{j r}^{i} \Gamma_{\mu}^{r} \longrightarrow \partial_{l}^{\prime} \Gamma_{\kappa}^{\prime k}+\Gamma_{l s}^{\prime k} \Gamma_{\kappa}^{\prime s}
$$

onde

$$
\begin{aligned}
\partial_{l}^{\prime} \Gamma_{\kappa}^{\prime k}+ & \Gamma_{l s}^{\prime k} \Gamma_{\kappa}^{\prime s} \\
= & \frac{\partial x^{\mu}}{\partial x^{\prime \kappa}} \frac{\partial q^{\prime k}}{\partial q^{i}} \frac{\partial q^{j}}{\partial q^{\prime l}} \partial_{j} \Gamma_{\mu}^{i}+\frac{\partial x^{\mu}}{\partial x^{\prime \kappa}} \frac{\partial^{2} q^{\prime k}}{\partial q^{j} \partial q^{i}} \frac{\partial q^{j}}{\partial q^{\prime l}} \Gamma_{\mu}^{i}+\frac{\partial x^{\mu}}{\partial x^{\prime \kappa}} \frac{\partial^{2} q^{\prime k}}{\partial x^{\mu} \partial q^{j}} \frac{\partial q^{j}}{\partial q^{\prime l}} \\
& +\left(\frac{\partial q^{\prime k}}{\partial q^{i}} \frac{\partial q^{j}}{\partial q^{\prime l}} \frac{\partial q^{r}}{\partial q^{\prime s}} \Gamma_{j r}^{i}-\frac{\partial^{2} q^{\prime k}}{\partial q^{j} \partial q^{r}} \frac{\partial q^{j}}{\partial q^{\prime l}} \frac{\partial q^{r}}{\partial q^{\prime s}}\right)\left(\frac{\partial x^{\mu}}{\partial x^{\prime \kappa}} \frac{\partial q^{\prime s}}{\partial q^{t}} \Gamma_{\mu}^{t}+\frac{\partial x^{\mu}}{\partial x^{\prime \kappa}} \frac{\partial q^{\prime s}}{\partial x^{\mu}}\right),
\end{aligned}
$$

isto é,

$$
\begin{aligned}
\partial_{l}^{\prime} \Gamma_{\kappa}^{\prime k}+ & \Gamma_{l s}^{\prime k} \Gamma_{\kappa}^{\prime s} \\
= & \frac{\partial x^{\mu}}{\partial x^{\prime \kappa}} \frac{\partial q^{\prime k}}{\partial q^{i}} \frac{\partial q^{j}}{\partial q^{\prime l}}\left(\partial_{j} \Gamma_{\mu}^{i}+\Gamma_{j r}^{i} \Gamma_{\mu}^{r}\right) \\
& +\frac{\partial x^{\mu}}{\partial x^{\prime \kappa}} \frac{\partial q^{j}}{\partial q^{\prime l}}\left(\frac{\partial q^{\prime k}}{\partial q^{i}} \frac{\partial q^{r}}{\partial q^{\prime s}} \frac{\partial q^{\prime s}}{\partial x^{\mu}} \Gamma_{j r}^{i}-\frac{\partial q^{r}}{\partial q^{\prime s}} \frac{\partial q^{\prime s}}{\partial x^{\mu}} \frac{\partial^{2} q^{\prime k}}{\partial q^{j} \partial q^{r}}+\frac{\partial^{2} q^{\prime k}}{\partial x^{\mu} \partial q^{j}}\right)
\end{aligned}
$$


Então

$$
e_{0} \longrightarrow e_{0}^{\prime}, e_{\mu}^{i} \longrightarrow e_{\kappa}^{\prime k}, e_{i} \longrightarrow e_{k}^{\prime}, e_{\mu} \longrightarrow e_{\kappa}^{\prime}
$$

onde

$$
e_{0}^{\prime}=\operatorname{det}\left(\frac{\partial x^{\prime}}{\partial x}\right) e_{0}
$$

De forma similar,

$$
\begin{aligned}
e_{\kappa}^{\prime k}= & \frac{\partial}{\partial p_{k}^{\prime \kappa}}-\Gamma_{k}^{\prime \kappa} \frac{\partial}{\partial p^{\prime}} \\
= & \operatorname{det}\left(\frac{\partial x^{\prime}}{\partial x}\right)\left(\frac{\partial x^{\mu}}{\partial x^{\prime \kappa}} \frac{\partial q^{\prime k}}{\partial q^{i}} \frac{\partial}{\partial p_{i}^{\mu}}+\frac{\partial x^{\mu}}{\partial x^{\prime \kappa}} \frac{\partial q^{\prime k}}{\partial x^{\mu}} \frac{\partial}{\partial p}\right) \\
& \quad-\operatorname{det}\left(\frac{\partial x^{\prime}}{\partial x}\right)\left(\frac{\partial x^{\mu}}{\partial x^{\prime \kappa}} \frac{\partial q^{\prime k}}{\partial q^{i}} \Gamma_{\mu}^{i}+\frac{\partial x^{\mu}}{\partial x^{\prime \kappa}} \frac{\partial q^{\prime k}}{\partial x^{\mu}}\right) \frac{\partial}{\partial p} \\
= & \operatorname{det}\left(\frac{\partial x^{\prime}}{\partial x}\right) \frac{\partial x^{\mu}}{\partial x^{\prime \kappa}} \frac{\partial q^{\prime k}}{\partial q^{i}}\left(\frac{\partial}{\partial p_{i}^{\mu}}-\Gamma_{\mu}^{i} \frac{\partial}{\partial p}\right),
\end{aligned}
$$

assim

$$
e_{\kappa}^{\prime k}=\operatorname{det}\left(\frac{\partial x^{\prime}}{\partial x}\right) \frac{\partial x^{\mu}}{\partial x^{\prime \kappa}} \frac{\partial q^{\prime k}}{\partial q^{i}} e_{\mu}^{i}
$$

Para uso posterior, observemos as seguintes leis de transformações, que são facilmente derivadas das equações (A.11) e (A.31):

$$
\begin{aligned}
& p_{r}^{\nu} e_{\nu}^{j} \longrightarrow p_{s}^{\prime \lambda} e_{\lambda}^{l l}=\frac{\partial q^{l}}{\partial q^{j}} \frac{\partial q^{r}}{\partial q^{\prime s}} p_{r}^{\nu} e_{\nu}^{j}, \\
& p_{j}^{\rho} e_{\nu}^{j} \longrightarrow p_{l}^{\prime \sigma} e_{\lambda}^{l l}=\frac{\partial x^{\nu}}{\partial x^{\prime \lambda}} \frac{\partial x^{\prime \sigma}}{\partial x^{p}} p_{j}^{\rho} e_{\nu}^{j}, \\
& p_{j}^{\nu} e_{\nu}^{j} \longrightarrow p_{l}^{\prime \lambda} e_{\lambda}^{l l}=p_{j}^{\nu} e_{\nu}^{j} .
\end{aligned}
$$

Agora podemos observar o comportamento do referencial por uma mudança de coordenadas com respeito as coordenadas $q$. Usamos as equações (A.6) e (A.7) para reescrevermo a equação (A.8) na forma

$$
e_{i}=\frac{\partial}{\partial q^{i}}+\Gamma_{i j}^{r} p_{r}^{\nu} e_{\nu}^{j}-\partial_{i} \Gamma_{\nu}^{r} p_{r}^{\nu} e_{0}
$$


e usando as leis de transformações de coordenadas estabelecidas previamente para calcular

$$
\begin{aligned}
e_{k}^{\prime}= & \frac{\partial}{\partial q^{\prime k}}+\Gamma_{k l}^{\prime s} p_{s}^{\prime \lambda} e_{\lambda}^{\prime j}-\partial_{k}^{\prime} \Gamma_{\lambda}^{\prime s} p_{s}^{\prime \lambda} e_{0}^{\prime} \\
= & \frac{\partial q^{i}}{\partial q^{\prime k}} \frac{\partial}{\partial q^{i}}+\frac{\partial^{2} q^{\prime s}}{\partial q^{i} \partial q^{r}} \frac{\partial q^{j}}{\partial q^{\prime s}} \frac{\partial q^{r}}{\partial q^{\prime k}} p_{j}^{\mu} \frac{\partial}{\partial p_{i}^{\mu}}+\frac{\partial^{2} q^{\prime s}}{\partial x^{\mu} \partial q^{r}} \frac{\partial q^{j}}{\partial q^{\prime s}} \frac{\partial q^{r}}{\partial q^{\prime k}} p_{j}^{\mu} \frac{\partial}{\partial p} \\
& +\frac{\partial q^{i}}{\partial q^{\prime k}} \frac{\partial q^{j}}{\partial q^{\prime \prime}}\left(\frac{\partial q^{\prime s}}{\partial q^{r}} \Gamma_{i j}^{r}-\frac{\partial^{2} q^{\prime s}}{\partial q^{i} \partial q^{j}}\right) \frac{\partial q^{\prime l}}{\partial q^{t}} \frac{\partial q^{u}}{\partial q^{\prime s}} p_{u}^{\nu} e_{\nu}^{t} \\
& -\frac{\partial q^{i}}{\partial q^{\prime k}}\left(\frac{\partial q^{\prime s}}{\partial q^{r}} \partial_{i} \Gamma_{\nu}^{r}+\frac{\partial^{2} q^{\prime s}}{\partial q^{i} \partial q^{r}} \Gamma_{\nu}^{r}+\frac{\partial^{2} q^{\prime s}}{\partial x^{\nu} \partial q^{i}}\right)\left(\frac{\partial q^{t}}{\partial q^{\prime s}} p_{t}^{\nu}\right) e_{0} \\
= & \frac{\partial q^{i}}{\partial q^{\prime k}}\left(\frac{\partial}{\partial q^{i}}+\Gamma_{i j}^{r} p_{r}^{\nu} e_{\nu}^{j}-\partial_{i} \Gamma_{\nu}^{r} p_{r}^{\nu} e_{0}\right) \\
& +\frac{\partial^{2} q^{\prime s}}{\partial q^{i} \partial q^{r}} \frac{\partial q^{j}}{\partial q^{\prime s}} \frac{\partial q^{r}}{\partial q^{\prime k}} p_{j}^{\mu} \frac{\partial}{\partial p_{i}^{\mu}}+\frac{\partial^{2} q^{\prime s}}{\partial x^{\mu} \partial q^{r}} \frac{\partial q^{j}}{\partial q^{\prime s}} \frac{\partial q^{r}}{\partial q^{\prime k}} p_{j}^{\mu} \frac{\partial}{\partial p} \\
& -\frac{\partial^{2} q^{\prime s}}{\partial q^{i} \partial q^{j}} \frac{\partial q^{i}}{\partial q^{\prime k}} \frac{\partial q^{u}}{\partial q^{\prime s}} p_{u}^{\nu} e_{\nu}^{j}-\left(\frac{\partial^{2} q^{\prime s}}{\partial q^{i} \partial q^{r}} \Gamma_{\nu}^{r}+\frac{\partial^{2} q^{\prime s}}{\partial x^{\nu} \partial q^{i}}\right) \frac{\partial q^{i}}{\partial q^{\prime k}} \frac{\partial q^{t}}{\partial q^{\prime s}} p_{t}^{\nu} e_{0}
\end{aligned}
$$

que fornece

$$
e_{k}^{\prime}=\frac{\partial q^{i}}{\partial q^{\prime k}} e_{i}
$$

Finalmente, para checarmos as leis de transformações para o referencial com respeito as coordenadas $x$, usamos as equações (A.6), (A.7) e (A.8) para reescrevermos a equação (A.9) na forma

$$
\begin{aligned}
e_{\mu}=\frac{\partial}{\partial x^{\mu}}+\Gamma_{\mu}^{j} e_{j} & -\left(\left(\partial_{j} \Gamma_{\mu}^{r}+\Gamma_{j i}^{r} \Gamma_{\mu}^{i}\right) p_{r}^{\nu}+\Gamma_{\mu \rho}^{\nu} p_{j}^{\rho}-\Gamma_{\mu \rho}^{\rho} p_{j}^{\nu}\right) e_{\nu}^{j} \\
& -\left(\partial_{\mu} \Gamma_{\nu}^{j} p_{j}^{\nu}-\Gamma_{\mu \rho}^{\rho}\left(p+\Gamma_{\nu}^{j} p_{j}^{\nu}\right)\right) e_{0}
\end{aligned}
$$

e usando as leis de transformações de coordenadas estabelecidas previamente calculamos 


$$
\begin{aligned}
& e_{\kappa}^{\prime}=\frac{\partial}{\partial x^{\prime \kappa}}+\Gamma_{\kappa}^{\prime l} e_{l}^{\prime}-\left(\left(\partial_{l}^{\prime} \Gamma_{\kappa}^{\prime s}+\Gamma_{l k}^{\prime s} \Gamma_{\kappa}^{\prime k}\right) p_{s}^{\prime \lambda}+\Gamma_{\kappa \sigma}^{\prime \lambda} p_{l}^{\prime \sigma}-\Gamma_{\kappa \sigma}^{\prime \sigma} p_{l}^{\prime \lambda}\right) e_{\lambda}^{\prime l} \\
& -\left(\partial_{\kappa}^{\prime} \Gamma_{\lambda}^{\prime l} p_{l}^{\prime \lambda}-\Gamma_{\kappa \sigma}^{\prime \sigma}\left(p^{\prime}+\Gamma_{\lambda}^{\prime l} p_{l}^{\prime \lambda}\right)\right) e_{0}^{\prime} \\
& =\frac{\partial x^{\mu}}{\partial x^{\prime \kappa}} \frac{\partial}{\partial x^{\mu}}-\frac{\partial x^{\mu}}{\partial x^{\prime k}} \frac{\partial q^{\prime k}}{\partial x^{\mu}} \frac{\partial q^{i}}{\partial q^{\prime k}} \frac{\partial}{\partial q^{i}} \\
& +\left(\frac{\partial x^{\rho}}{\partial x^{\prime \sigma}} \frac{\partial^{2} x^{\prime \sigma}}{\partial x^{\nu} \partial x^{\rho}} \frac{\partial x^{\nu}}{\partial x^{\prime \kappa}} p_{i}^{\mu}-\frac{\partial x^{\mu}}{\partial x^{\prime \sigma}} \frac{\partial^{2} x^{\prime \sigma}}{\partial x^{\nu} \partial x^{\rho}} \frac{\partial x^{\rho}}{\partial x^{\prime \kappa}} p_{i}^{\nu}\right. \\
& \left.+\frac{\partial x^{\nu}}{\partial x^{\prime \kappa}} \frac{\partial^{2} q^{\prime k}}{\partial x^{\nu} \partial q^{i}} \frac{\partial q^{j}}{\partial q^{\prime k}} p_{j}^{\mu}-\frac{\partial x^{\nu}}{\partial x^{\prime \kappa}} \frac{\partial q^{\prime k}}{\partial x^{\nu}} \frac{\partial q^{r}}{\partial q^{\prime k}} \frac{\partial^{2} q^{\prime l}}{\partial q^{i} \partial q^{r}} \frac{\partial q^{j}}{\partial q^{\prime l}} p_{j}^{\mu}\right) \frac{\partial}{\partial p_{i}^{\mu}} \\
& +\frac{\partial x^{\mu}}{\partial x^{\prime k}}\left(\frac{\partial^{2} q^{\prime k}}{\partial x^{\mu} \partial x^{\nu}} \frac{\partial q^{i}}{\partial q^{\prime k}} p_{i}^{\nu}-\frac{\partial q^{\prime l}}{\partial x^{\mu}} \frac{\partial q^{r}}{\partial q^{\prime l}} \frac{\partial^{2} q^{\prime k}}{\partial x^{\nu} \partial q^{r}} \frac{\partial q^{i}}{\partial q^{\prime k}} p_{i}^{\nu}\right. \\
& \left.-\frac{\partial^{2} x^{\prime \sigma}}{\partial x^{\mu} \partial x^{\nu}} \frac{\partial x^{\rho}}{\partial x^{\prime \sigma}} \frac{\partial q^{\prime k}}{\partial x^{\rho}} \frac{\partial q^{i}}{\partial q^{\prime k}} p_{i}^{\nu}+\frac{\partial^{2} x^{\prime \sigma}}{\partial x^{\mu} \partial x^{\rho}} \frac{\partial x^{\rho}}{\partial x^{\prime \sigma}} p\right) \frac{\partial}{\partial p} \\
& +\left(\frac{\partial x^{\mu}}{\partial x^{\prime \kappa}} \frac{\partial q^{\prime l}}{\partial q^{j}} \Gamma_{\mu}^{j}+\frac{\partial x^{\mu}}{\partial x^{\prime \kappa}} \frac{\partial q^{\prime l}}{\partial x^{\mu}}\right) \frac{\partial q^{i}}{\partial q^{\prime l}} e_{i} \\
& -\frac{\partial x^{\mu}}{\partial x^{\prime \kappa}} \frac{\partial q^{j}}{\partial q^{\prime l}}\left(\frac{\partial q^{\prime s}}{\partial q^{r}}\left(\partial_{j} \Gamma_{\mu}^{r}+\Gamma_{j i}^{r} \Gamma_{\mu}^{i}\right)+\frac{\partial q^{\prime s}}{\partial q^{r}} \frac{\partial q^{i}}{\partial q^{\prime k}} \frac{\partial q^{\prime k}}{\partial x^{\mu}} \Gamma_{j i}^{r}\right. \\
& \left.-\frac{\partial q^{i}}{\partial q^{\prime k}} \frac{\partial q^{\prime k}}{\partial x^{\mu}} \frac{\partial^{2} q^{\prime s}}{\partial q^{j} \partial q^{i}}+\frac{\partial^{2} q^{\prime s}}{\partial x^{\mu} \partial q^{j}}\right) \frac{\partial q^{l}}{\partial q^{t}} \frac{\partial q^{u}}{\partial q^{\prime s}} p_{u}^{\nu} e_{\nu}^{t} \\
& -\left(\frac{\partial x^{\prime \lambda}}{\partial x^{\nu}} \frac{\partial x^{\mu}}{\partial x^{\prime \kappa}} \frac{\partial x^{\rho}}{\partial x^{\prime \sigma}} \Gamma_{\mu \rho}^{\nu}-\frac{\partial^{2} x^{\prime \lambda}}{\partial x^{\mu} \partial x^{\rho}} \frac{\partial x^{\mu}}{\partial x^{\prime \kappa}} \frac{\partial x^{\rho}}{\partial x^{\prime \sigma}}\right) \frac{\partial x^{\beta}}{\partial x^{\prime \lambda}} \frac{\partial x^{\prime \sigma}}{\partial x^{\delta}} p_{j}^{\delta} e_{\beta}^{j} \\
& +\frac{\partial x^{\mu}}{\partial x^{\prime \kappa}}\left(\Gamma_{\mu \rho}^{\rho}-\frac{\partial^{2} x^{\prime \sigma}}{\partial x^{\mu} \partial x^{\rho}} \frac{\partial x^{\rho}}{\partial x^{\prime \sigma}}\right)\left(p_{j}^{\nu} e_{\beta}^{j}+\left(p+\Gamma_{\nu}^{j} p_{j}^{\nu}\right) e_{0}\right) \\
& -\frac{\partial x^{\mu}}{\partial x^{\prime \kappa}}\left(\frac{\partial q^{\prime l}}{\partial q^{j}} \tilde{\partial}_{\mu} \Gamma_{\nu}^{j}+\frac{\partial^{2} q^{\prime l}}{\partial x^{\mu} \partial q^{j}} \Gamma_{\nu}^{j}-\frac{\partial^{2} x^{\prime \sigma}}{\partial x^{\nu} \partial x^{\mu}} \frac{\partial x^{\rho}}{\partial x^{\prime \sigma}} \frac{\partial q^{\prime l}}{\partial q^{j}} \Gamma_{\rho}^{j}\right. \\
& +\frac{\partial^{2} q^{\prime \prime}}{\partial x^{\nu} \partial x^{\mu}}-\frac{\partial^{2} x^{\prime \sigma}}{\partial x^{\nu} \partial x^{\mu}} \frac{\partial x^{\rho}}{\partial x^{\prime \sigma}} \frac{\partial q^{\prime l}}{\partial x^{\rho}} \\
& \left.-\frac{\partial q^{\prime k}}{\partial x^{\mu}} \frac{\partial q^{i}}{\partial q^{\prime k}}\left(\frac{\partial q^{\prime l}}{\partial q^{j}} \partial_{i} \Gamma_{\nu}^{j}+\frac{\partial^{2} q^{\prime l}}{\partial q^{i} \partial q^{j}} \Gamma_{\nu}^{j}+\frac{\partial^{2} q^{\prime l}}{\partial x^{\nu} \partial q^{i}}\right)\right) \\
& \times \frac{\partial q^{t}}{\partial q^{\prime l}} p_{i}^{\nu} e_{0}
\end{aligned}
$$


De acordo com a equação (A.35), os termos sublinhados se cancelam, e após renomear os indíces em termos originados da equação (A.18), temos que

$$
\begin{aligned}
& e_{\kappa}^{\prime}=\frac{\partial x^{\mu}}{\partial x^{\prime \kappa}}\left[\frac{\partial}{\partial x^{\mu}}+\Gamma_{\mu}^{j} e_{j}-\left(\left(\partial_{j} \Gamma_{\mu}^{r}+\Gamma_{j i}^{r} \Gamma_{\mu}^{i}\right) p_{r}^{\nu}+\Gamma_{\mu \rho}^{\nu} p_{j}^{\rho}-\Gamma_{\mu \rho}^{\rho} p_{j}^{\nu}\right) e_{\nu}^{j}\right. \\
& \left.-\left(\partial_{\mu} \Gamma_{\nu}^{j} p_{j}^{\nu}-\Gamma_{\mu \rho}^{\rho}\left(p+\Gamma_{\nu}^{j} p_{j}^{\nu}\right)\right) e_{0}\right] \\
& +\underbrace{\frac{\partial x^{\rho}}{\partial x^{\prime \sigma}} \frac{\partial^{2} x^{\prime \sigma}}{\partial x^{\mu} \partial x^{\rho}} \frac{\partial x^{\mu}}{\partial x^{\prime \kappa}} p_{j}^{\nu} \frac{\partial}{\partial p_{j}^{\nu}}}_{1}-\underbrace{\frac{\partial x^{\nu}}{\partial x^{\prime \lambda}} \frac{\partial^{2} x^{\prime \lambda}}{\partial x^{\rho} \partial x^{\mu}} \frac{\partial x^{\mu}}{\partial x^{\prime \kappa}} p_{j}^{\rho} \frac{\partial}{\partial p_{j}^{\nu}}}_{2} \\
& +\underbrace{\frac{\partial x^{\mu}}{\partial x^{\prime \kappa}} \frac{\partial^{2} q^{\prime s}}{\partial x^{\mu} \partial q^{j}} \frac{\partial q^{r}}{\partial q^{\prime s}} p_{r}^{\nu} \frac{\partial}{\partial p_{j}^{\nu}}}_{3}-\underbrace{\frac{\partial x^{\mu}}{\partial x^{\prime \kappa}} \frac{\partial q^{\prime k}}{\partial x^{\mu}} \frac{\partial q^{i}}{\partial q^{\prime k}} \frac{\partial^{2} q^{\prime s}}{\partial q^{j} \partial q^{i}} \frac{\partial q^{r}}{\partial q^{\prime s}} p_{r}^{\nu} \frac{\partial}{\partial p_{j}^{\nu}}}_{4} \\
& +\frac{\partial x^{\mu}}{\partial x^{\prime \kappa}}(\underbrace{\frac{\partial^{2} q^{\prime l}}{\partial x^{\mu} \partial x^{\nu}} \frac{\partial q^{j}}{\partial q^{\prime l}} p_{j}^{\nu}}_{5}-\underbrace{\frac{\partial q^{\prime k}}{\partial x^{\mu}} \frac{\partial q^{i}}{\partial q^{\prime k}} \frac{\partial^{2} q^{\prime l}}{\partial x^{\nu} \partial q^{i}} \frac{\partial q^{j}}{\partial q^{\prime l}} p_{j}^{\nu}}_{6} \\
& -\underbrace{\frac{\partial^{2} x^{\prime \sigma}}{\partial x^{\mu} \partial x^{\nu}} \frac{\partial x^{\rho}}{\partial x^{\prime \sigma}} \frac{\partial q^{\prime l}}{\partial x^{\rho}} \frac{\partial q^{j}}{\partial q^{\prime l}} p_{j}^{\nu}}_{7}+\underbrace{\frac{\partial^{2} x^{\prime \sigma}}{\partial x^{\mu} \partial x^{\rho}} \frac{\partial x^{\rho}}{\partial x^{\prime \sigma}} p}_{8}) \frac{\partial}{\partial p} \\
& +\underbrace{\frac{\partial x^{\mu}}{\partial x^{\prime \kappa}} \frac{\partial q^{i}}{\partial q^{\prime k}} \frac{\partial q^{\prime k}}{\partial x^{\mu}} \frac{\partial^{2} q^{\prime s}}{\partial q^{j} \partial q^{i}} \frac{\partial q^{r}}{\partial q^{\prime s}} p_{r}^{\nu} e_{\nu}^{j}}_{4}-\underbrace{\frac{\partial x^{\mu}}{\partial x^{\prime \kappa}} \frac{\partial^{2} q^{\prime s}}{\partial x^{\mu} \partial q^{j}} \frac{\partial q^{r}}{\partial q^{\prime s}} p_{r}^{\nu} e_{\nu}^{j}}_{3} \\
& +\underbrace{\frac{\partial x^{\mu}}{\partial x^{\prime \kappa}} \frac{\partial^{2} x^{\prime \lambda}}{\partial x^{\mu} \partial x^{\rho}} \frac{\partial x^{\nu}}{\partial x^{\prime \lambda}} p_{j}^{\rho} e_{\nu}^{j}}_{2}-\frac{\partial x^{\mu}}{\partial x^{\prime \kappa}} \frac{\partial^{2} x^{\prime \sigma}}{\partial x^{\mu} \partial x^{\rho}} \frac{\partial x^{\rho}}{\partial x^{\prime \sigma}}(\underbrace{p_{j}^{\nu} e_{\nu}^{j}+\Gamma_{\nu}^{j} p_{j}^{\nu} e_{0}}_{1}+\underbrace{p e_{0}}_{8}) \\
& -\frac{\partial x^{\mu}}{\partial x^{\prime \kappa}} \frac{\partial q^{j}}{\partial q^{l l}}(\underbrace{\frac{\partial^{2} q^{\prime l}}{\partial x^{\mu} \partial q^{i}} \Gamma_{\nu}^{i}}_{3}-\underbrace{\frac{\partial^{2} x^{\prime \sigma}}{\partial x^{\nu} \partial x^{\mu}} \frac{\partial x^{\rho}}{\partial x^{\prime \sigma}} \frac{\partial q^{\prime l}}{\partial q^{i}} \Gamma_{\rho}^{i}}_{2} \\
& +\underbrace{\frac{\partial^{2} q^{\prime l}}{\partial x^{\nu} \partial x^{\mu}}}_{5}-\underbrace{\frac{\partial^{2} x^{\prime \sigma}}{\partial x^{\nu} \partial x^{\mu}} \frac{\partial x^{\rho}}{\partial x^{\prime \sigma}} \frac{\partial q^{\prime l}}{\partial x^{\rho}}}_{7} \\
& -\underbrace{\frac{\partial q^{\prime k}}{\partial x^{\mu}} \frac{\partial q^{i}}{\partial q^{\prime k}} \frac{\partial^{2} q^{\prime l}}{\partial q^{i} \partial q^{r}} \Gamma_{\nu}^{r}}_{4}-\underbrace{\frac{\partial q^{\prime k}}{\partial x^{\mu}} \frac{\partial q^{i}}{\partial q^{\prime k}} \frac{\partial^{2} q^{\prime l}}{\partial x^{\nu} \partial q^{i}}}_{6}) p_{j}^{\nu} e_{0} .
\end{aligned}
$$


Todos os termos não homogeneos se cancelam, e ficamos com

$$
e_{\kappa}^{\prime}=\frac{\partial x^{\mu}}{\partial x^{\prime \kappa}} e_{\mu}
$$




\section{Bibliografia}

[1] R. Abraham \& J.E. Marsden: Foundations of Mechanics, $2^{\text {nd }}$ edition, Benjamin/Cummings, Reading 1978.

[2] V. ARnold: Mathematical Methods of Classical Mechanics, $2^{\text {nd }}$ edition, Springer, Berlin 1989.

[3] J.F. Cariñena, M. Crampin \& L.A. Ibort: On the Multisymplectic Formalism for First Order Field Theories, Diff. Geom. Appl. 1 (1991) 345-374.

[4] C. CRnković \& E. Witten: Covariant Description of Canonical Formalism in Geometrical Theories, in: "Three Hundred Years of Gravitation", pp. 676-684, eds: W. Israel \& S. Hawking, Cambridge University Press, Cambridge 1987.

[5] C. CRnković: Symplectic Geometry of Covariant Phase Space, Class. Quantum Grav. 5 (1988) 1557-1575.

[6] Th. De Donder: Théorie Invariante du Calcul des Variations, Gauthier-Villars, Paris 1935.

[7] B. DeWitt: Invariant Commutators for the Quantized Gravitational Field, Phys. Rev. Lett. 4 (1960) 317-320.

[8] B. DeWitT: Dynamical Theory of Groups and Fields, in: "Relativity, Groups and Topology" (1963 Les Houches Lectures), pp. 585-820, eds: B. DeWitt \& C. DeWitt, Gordon and Breach, New York 1964.

[9] B. DeWItT: The Spacetime Approach to Quantum Field Theory, in: "Relativity, Groups and Topology II" (1983 Les Houches Lectures), pp. 382-738, eds: B. DeWitt \& R. Stora, Elsevier, Amsterdam 1984. 
[10] A. Echeverría-Enríouez, M.C. Muñoz-LeCAnda: Geometry of Multisymplectic Hamiltonian First-Order Field Theories, J. Math. Phys. 41 (2000) 7402-7444, $\mathrm{dg}-\mathrm{ga} / 0004005$.

[11] M. Forger \& H. Römer: The Poisson Bracket on Multisymplectic Phase Space, Rep. Math. Phys. 48 (2001) 211-218, math-ph/0009037.

[12] M. Forger, C. Paufler \& H. Römer: The Poisson Bracket for Poisson Forms in Multisymplectic Field Theory, Rev. Math. Phys. 15 (2003) 705-743, math-ph/0202043.

[13] M. Forger, C. Paufler \& H. Römer: Hamiltonian Multivector Fields and Poisson Forms in Multisymplectic Field Theory, submetido para publicação, math-ph/0407057.

[14] M. Forger \& S.V. Romero: Covariant Poisson Brackets in Geometric Field Theory, aceito para publicação em Commun. Math. Phys. math-ph/0408008.

[15] H. Goldschmidt and S. Sternberg: The Hamilton-Cartan Formalism in the Calculus of Variations, Ann. Inst. Fourier 23 (1973) 203-267.

[16] M. J. Gotay: A multisymplectic framework for classical field theory and the calculus of variations. I: Covariant Hamiltonian formalism, in: "Mechanics, Analysis and Geometry: 200 years after Lagrange", pp. 203-235, eds: M. Francaviglia \& D.D. Holm, North-Holland, Amsterdam 1991.

[17] M.J. Gotay, J. Isenberg \& J.E. Marsden: Momentum Maps and Classical Relativistic Fields I: Covariant Field Theory, physics/9801019.

[18] V. Guillemin and S. Sternberg: Geometric Asymptotics, AMS, Providence 1977.

[19] W.H. Greub, S. Halperin \& R. Vanstone: Connections, Curvature and Cohomology, Vol. 2, Academic Press, New York 1973.

[20] I.V. Kanatchikov: Canonical Structure of Classical Field Theory in the Polymomentum Phase Space, Rep. Math. Phys. 41 (1998) 49-90, hep-th/9709229.

[21] J. Kijowski: A Finite-Dimensional Canonical Formalism in the Classical Field Theory, Commun. Math. Phys. 30 (1973) 99-128; Multiphase Spaces and Gauge in the Calculus of Variations, Bull. Acad. Sc. Polon. 22 (1974) 1219-1225.

[22] S. Kobayashi \& K. Nomizu: Foundations of Differential Geometry, Vol. 1, Interscience, New York 1963. 
[23] J. Kijowski \& W. Szczyrba: Multisymplectic Manifolds and the Geometrical Construction of the Poisson Brackets in the Classical Field Theory, in: "Géometrie Symplectique et Physique Mathématique", pp. 347-379, ed.: J.-M. Souriau, C.N.R.S., Paris 1975.

[24] J. Kijowski \& W. Szczyrba: A Canonical Structure for Classical Field Theories, Commun. Math. Phys. 46 (1976) 183-206.

[25] J. Kijowski \& W. Tulczyjew: A Symplectic Framework for Field Theories, Lecture Notes in Physics; Vol. 107, Springer, Berlin 1979.

[26] I. Kolár̆, P.W. Michor \& J. Slovák: Natural Operations in Differential Geometry, Springer, Berlin 1993.

[27] S. LANG: Differential Manifolds, Addison-Wesley, Reading 1972.

[28] L. Mangiarotti, G. Sardanashvily: Connections in Classical and Quantum field Theory, World Scientific, Singapore 2000.

[29] G. Martin: A Darboux Theorem for Multisymplectic Manifolds, Lett. Math. Phys. 16 (1988) 133-138.

[30] G. Martin: Dynamical Structures for k-Vector Fields, Int. J. Theor. Phys. 41 (1988) 571-585.

[31] M. Modugno \& A.M. Vianagrodov: Some Variations on the Notion of Connection. Annali di Matematica Pura ed Aplicata (IV) , 167 (1984) 33-71.

[32] R.E. Peierls: The Commutation Laws of Relativistic Field Theory, Proc. Roy. Soc. Lond. A 214 (1952) 143-157.

[33] C. Paufler, H. RÖmer: Geometry of Hamiltonian n-vectors in Multisymplectic Field Theory, J. Geom. Phys. 44 (2002) 52-69, math-ph/0102008.

[34] S.V. Romero: Colchete de Poisson Covariante na Teoria Geométrica dos Campos, Tese de Doutorado, Instituto de Matemática e Estatística, Universidade de São Paulo, Junho de 2001.

[35] D. Saunders: The Geometry of Jet Bundles, Springer, Berlin 1993.

[36] B. Reinhart: Differential Geometry and Foliations, Springer, Berlin 1983.

[37] W.M. Tulczyjew: The Graded Lie Algebra of Multivector Fields and the Generalized Lie Derivative of Forms, Bull. Acad. Pol. Sci. SMAP 22 (1974) 937-942. 
[38] R.M. WaLD: General Relativity; Chicago University Press, Chicago 1984.

[39] H. Weyl: Geodesic Fields in the Calculus of Variations for Multiple Integrals, Ann. Math. 36 (1935) 607-629.

[40] N.M.J. Woodhouse: Geometric Quantization, $2^{\text {nd }}$ edition, Oxford University Press, Oxford 1992.

[41] G. Zuckerman: Action Principles and Global Geometry, in: "Mathematical Aspects of String Theory", pp. 259-288, ed.: S.-T. Yau, World Scientific, Singapore 1987. 